\title{
Improving diagnostics and quality of life in children with peanut allergy and other atopic diseases
}

Hannah Kansen

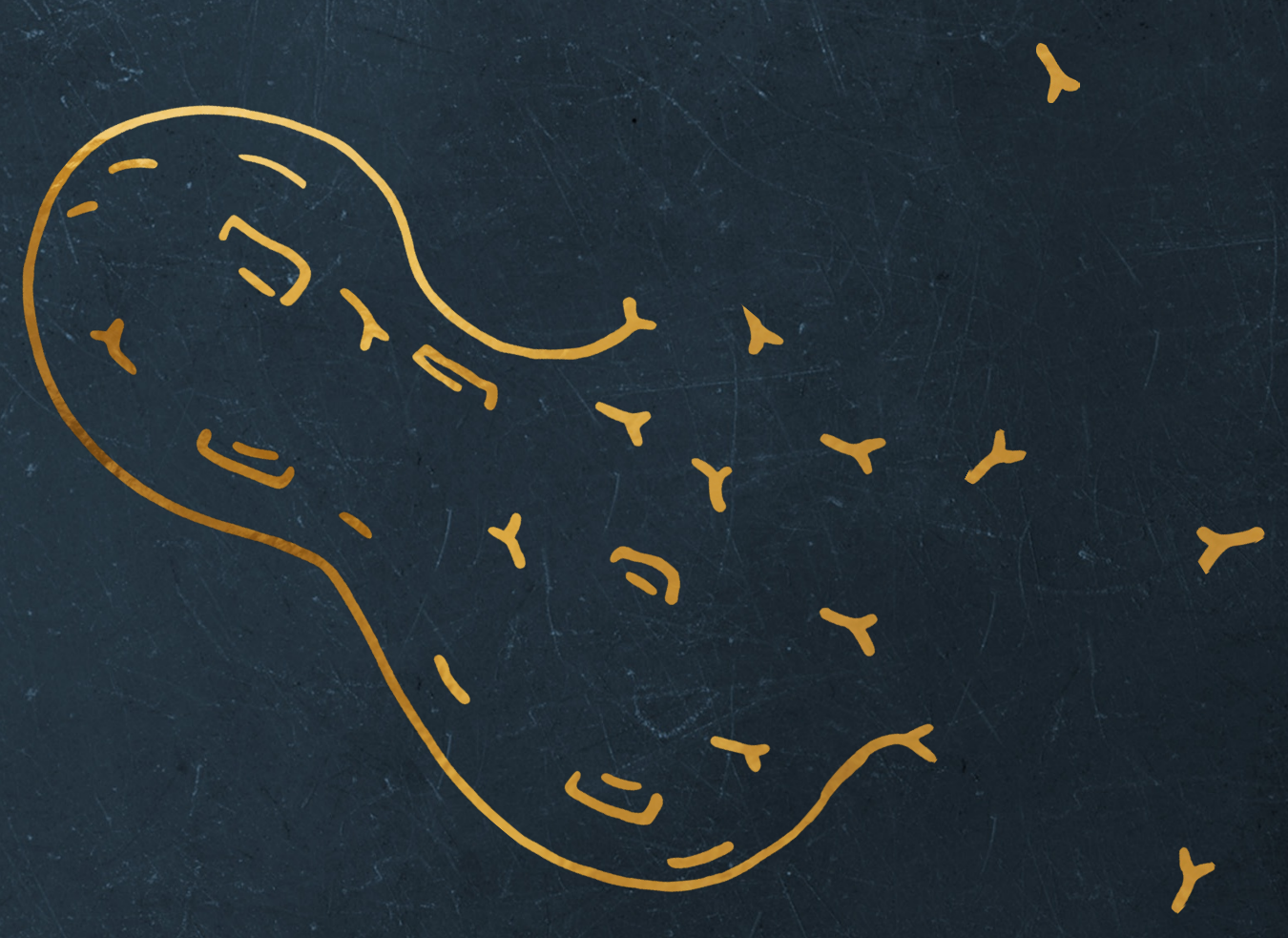





\section{Improving diagnostics and quality of life in children with peanut allergy and other atopic diseases}


Improving diagnostics and quality of life in children with peanut allergy and other atopic diseases

PhD thesis, Utrecht University, the Netherlands

(c) Hannah Kansen, 2020, Utrecht, the Netherlands

All rights reserved. No part of this thesis may be reproduced, stored in a retrieval system, or transmitted in any form or by any means, electronically, mechanically, by photo-copying, recording or otherwise, without prior written permission of the author. The copyright of the papers that have been published has been transferred to the respective publishers.

ISBN

Cover

Lay-out

Printed by
978-90-393-7302-6

Hannah Kansen and Erik van den Berg Wallpaper designed by rawpixel.com/freepik Hannah Kansen proefschriftmaken.nl 


\section{Improving diagnostics and quality of life in children with peanut allergy and other atopic diseases}

Het verbeteren van de diagnostiek en kwaliteit van leven van

kinderen met pinda allergie en andere atopische ziekten

(met een samenvatting in het Nederlands)

\section{Proefschrift}

ter verkrijging van de graad van doctor aan de Universiteit Utrecht op gezag van de rector magnificus, prof. dr. H.R.B.M. Kummeling, ingevolge het besluit van het college voor promoties in het openbaar te verdedigen op donderdag 27 augustus 2020 des ochtends te 10.30 uur

door

\section{Hannah Maria Kansen}

geboren op 20 mei 1991 te Hilversum 


\section{Promotoren}

Prof. dr. C.K. van der Ent

Prof. dr. A.C. Knulst

\section{Copromotoren}

Dr. F.C. van Erp

Dr. T.T.M. Le 


\section{Contents}

Chapter 1 General introduction

Part I. Improving diagnostics in patients with atopic diseases

Chapter 2 A diagnostic flowchart for pediatric peanut allergy

using Ara h 2 is accurate, safe and reduces costs

Chapter 3 Accurate prediction of peanut allergy in one third

of adults using a validated Ara h 2 cutoff

Chapter 4 Three-year follow-up after peanut food challenges:

accidental reactions in allergic children and

introduction failure in tolerant children

Chapter 5 Risk factors for atopic diseases and recurrent respiratory tract infections in children

\section{Part II. Improving quality of life in patients with atopic diseases}

Chapter 6 The impact of oral food challenges for food allergy on quality of life: a systematic review

Chapter 7 Prevalence and predictors of uncontrolled asthma in children referred for asthma and other atopic diseases

Chapter 8 Perceived triggers of asthma impair quality of life in children with asthma

Chapter 9 General discussion and summary

Chapter 10 Nederlandse samenvatting

$\begin{array}{lll}\text { Chapter } 11 & \text { List of abbreviations } & 228\end{array}$

Contributing authors $\quad 230$

Dankwoord $\quad 234$

List of publications 236

$\begin{array}{ll}\text { Curriculum vitae } & 238\end{array}$ 


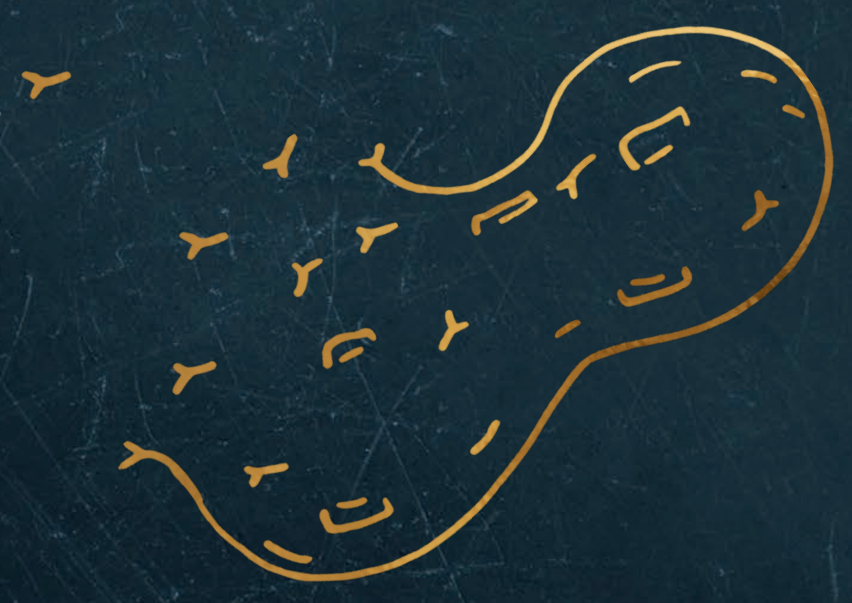




\section{Chapter 1}

General introduction 


\section{Atopic diseases}

The word atopy was first introduced by American allergists A.F. Coca and R.A. Cooke in 1922. ${ }^{1}$ Atopy (Greek atomos, meaning out of place or unusual) refers to the genetic tendency to produce immunoglobulin $\mathrm{E}$ (IgE) antibodies (sensitization) in response to common environmental proteins i.e. food allergens or inhalant allergens. ${ }^{2}$ Patients with atopy can develop one or more atopic disease(s) including food allergy, asthma, allergic rhinitis and atopic dermatitis. The prevalence of atopic diseases has been on the rise over the past decades. ${ }^{3,4}$ The point prevalence of childhood food allergy has been estimated at approximately $6-8 \% \%^{5,6}$, asthma at 8$35 \%^{3}$, allergic rhinitis at $7-19 \%^{3}$ and atopic dermatitis at $4-17 \%^{3}$. The progression of atopic diseases during childhood is often referred to as the 'atopic march'. Typically, the atopic march starts with atopic dermatitis, followed by food allergy, asthma and allergic rhinitis. ${ }^{7}$ The presence of one atopic disease increases the risk for the development of other atopic diseases and as a consequence, children often have multiple atopic diseases. ${ }^{8}$ As an example, the presence of atopic comorbidities in children with food allergy included in the Electronic Portal is shown in Figure 1. The Electronic Portal is a web-based application with multiple validated questionnaires administered to (parents of) children with respiratory or allergic symptoms, as previously published in detail. ${ }^{9}$ In this thesis, we focus mainly on improving diagnostics (part I) and quality of life (part II) in patients with (suspected) peanut allergy and in patients with asthma.

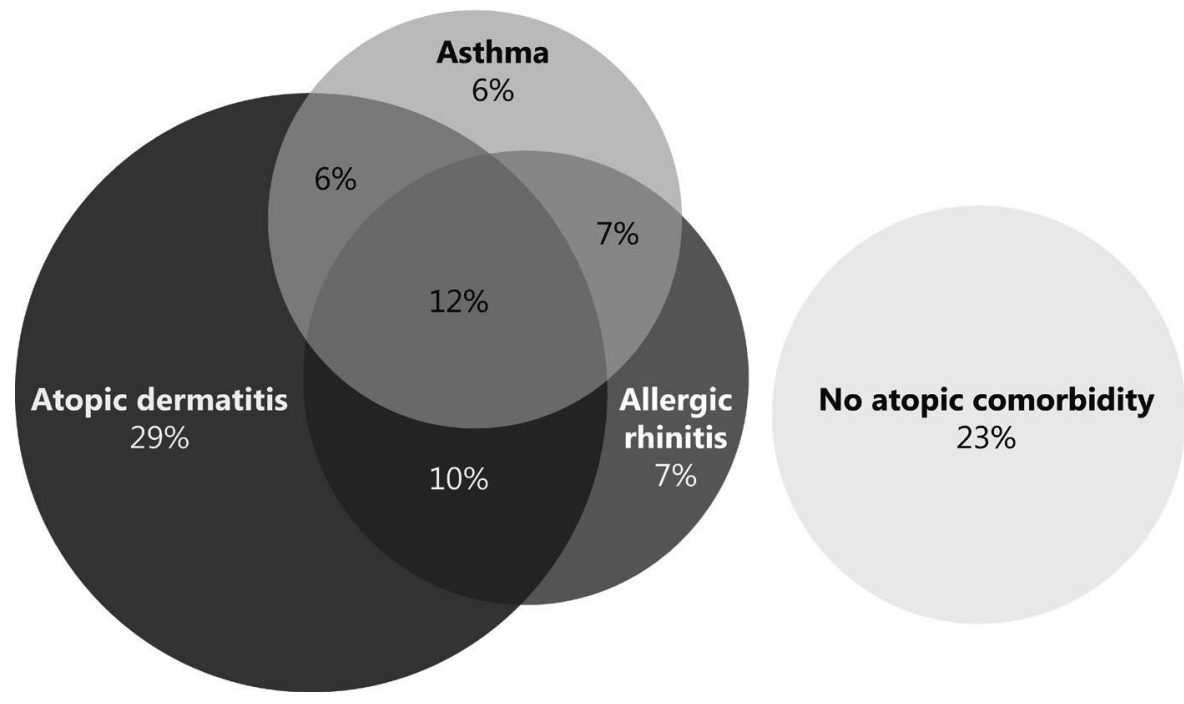

Figure 1. Presence of asthma, allergic rhinitis and atopic dermatitis in children with food allergy included in the Electronic Portal 


\section{The diagnostics of atopic diseases}

'The three main tasks of the clinician are diagnosis, prognosis and treatment. Of these, diagnosis is by far the most important for upon it the success of the other two depends.'

J.A. Ryle ${ }^{10}$

Historically, diagnosis, prognosis and treatment are the cornerstones of clinical practice. ${ }^{11}$ The purpose of a disease diagnosis is to categorize patients into groups with similar characteristics and thereafter, make inferences about the patients' prognosis and response to treatment (when available). An accurate diagnosis is important to adequately treat and advice patients, and to prevent unwanted events such as allergic reactions in patients with food allergy and daily symptoms or exacerbations in patients with asthma. Furthermore, an accurate diagnosis may improve health-related quality of life and reduce anxiety. ${ }^{12-14}$

\section{Peanut allergy}

In the first part of this thesis, we focus on how to improve the diagnostic pathway for peanut allergy. Peanut allergy is one of the most common food allergies worldwide affecting $0.2-2.2 \%$ of children and up to $0.6 \%$ of adults. ${ }^{15-17}$ Patients are suspected of having a peanut allergy when allergic symptoms (i.e. skin, gastrointestinal, respiratory, cardiovascular and/or neurological symptoms) occur immediately after ingestion of peanut together with sensitization to peanut. ${ }^{18}$ Sensitization can be detected by a positive skin prick test (SPT) or a raised level of specific IgE (sIgE) to peanut extract or peanut components in the blood. Currently, a two-day double-blind placebo-controlled food challenge (DBPCFC) is the reference standard for the definitive diagnosis of peanut allergy. ${ }^{18}$ However, a DBPCFC is time-consuming, expensive and subjective clinical symptoms can be difficult to interpret or severe systemic symptoms can occur. ${ }^{18,19}$

In recent years, the diagnostic work-up for patients with a suspected peanut or hazelnut allergy has been improved with the use of component-resolved diagnostics (CRD). ${ }^{20,21}$ CRD measures sIgE against individual allergenic proteins utilizing purified native or recombinant allergenic molecules. Sensitization to peanut component Ara h 2 and Ara h 6 demonstrates the best association with true peanut allergy in children and adults. ${ }^{22-27}$ Our research group previously found that $62 \%$ of children could be classified correctly when using sIgE to Ara h 2 cutoff levels with $100 \%$ negative and positive predictive values (cutoff levels at $\leq 0.1$ and $\geq 5.0$ $\mathrm{kU}_{\mathrm{A}} / \mathrm{L}$, respectively). When using a diagnostic flowchart based on these Ara $\mathrm{h} 2$ cutoff levels, the number and days of DBPCFCs could be reduced..$^{28}$ Additionally, 
the use of a diagnostic flowchart might result in lower costs. Previous research of a model-based economic evaluation in children with peanut allergy showed that a diagnostic flowchart using component-resolved diagnostics was more costeffective than current clinical practice including an oral food challenge. ${ }^{29}$ To date, no studies investigated the validation of previously published cutoff levels of sIgE to peanut components and the diagnostic accuracy, safety, adherence and costs of a diagnostic flowchart based on component-resolved diagnostics in daily practice. Therefore, we implemented a diagnostic flowchart for peanut allergy in one tertiary care and three secondary care centers in the Netherlands and evaluated these aspects in chapter 2. Furthermore, we evaluated whether we could develop a diagnostic flowchart based on component-resolved diagnostics in adults with suspected peanut allergy in chapter 3 .

\section{Atopic diseases and recurrent respiratory tract infections}

While the prevalence of atopic diseases has been rising in the past decades, the prevalence of infectious diseases has decreased. ${ }^{5,30-34}$ These opposing trends in prevalence have been linked and it has been suggested that a decline in infections and thereby lower microbial exposure could contribute to the increased prevalence of atopic diseases. In support of this hypothesis, a reduced risk of allergic sensitization has been observed in children with older siblings, in children who attended day care during infancy or in children living in a rural environment (i.e. high microbial exposure). ${ }^{35}$ On the contrary, an increased risk of recurrent infections has been observed in children with older siblings, in children who used a pacifier or in children who attended day care. ${ }^{36-38}$ Thus, (combined) environmental factors might affect the development of atopic and infectious diseases in different directions. In addition, the interaction between genetic and environmental factors is thought to be important in the pathogenesis of atopic diseases. ${ }^{39}$ However, it is unknown whether these interactions are disease-specific or associated with all atopic diseases. ${ }^{40}$ For respiratory infections, no consistent associations are observed regarding host genetic factors. ${ }^{41}$

Insight into how environmental and genetic (e.g. family history) factors are associated with atopic diseases on the one hand and infections on the other hand could improve our understanding on the pathophysiology of these diseases. We collected a large set of data on demographic, environmental and family history risk factors for atopic diseases and recurrent respiratory tract infections in children and investigated whether the associations between these risk factors with atopic diseases differed from the associations with recurrent respiratory tract infections (chapter 5). 


\section{The impact of atopic diseases on patients' welfare}

'Health is a state of complete physical, mental and social well-being and not merely the absence of disease or infirmity.'

World Health Organization, 1948

Diagnostic modalities mainly focus on a single organ, but it is likely that diagnostic strategies have a broader impact. In this regard, outcome measures that reflect the overall wellbeing of patients are relevant. The patients' perspective on several domains of life, including physical, mental and social health, can be measured using health-related quality of life questionnaires. ${ }^{42}$ The concept of health-related quality of life (HRQL) is rooted in the definition of health by the World Health Organization. HRQL can be measured using generic or disease-specific questionnaires. Generic HRQL questionnaires facilitate comparison between different diseases and disease-specific HRQL questionnaires reflect disease-related impairments. Previous research using generic HRQL questionnaires showed that children with atopic diseases have impaired quality of life compared to the general population. ${ }^{43,44}$ Further insight into the impact of atopic diseases on patients' welfare can be gained by assessing anxiety symptoms. Previous studies indicated that anxiety symptoms are highly prevalent in children with atopic diseases, especially in children with multiple atopic diseases. ${ }^{45,46}$

\section{Peanut allergy}

Food allergy is detrimental to patients' welfare due to the constant vigilance to avoid food allergens and the fear of accidental allergic reactions. ${ }^{47}$ Children with peanut allergy experience more impairments in HRQL compared with children with other food allergies. ${ }^{48}$ Peanut allergy may have profound impact on anxiety and $\mathrm{HRQL}$ as symptoms can be severe and the amount of peanut triggering an allergic reaction is often minute. ${ }^{49,50}$ In parents of children with peanut allergy, considerable levels of anxiety have been reported. ${ }^{51}$ Moreover, increased levels of anxiety have been reported in parents of children with suspected food allergy prior to a definitive food allergy diagnosis. ${ }^{52}$

Previous research suggests that an oral food challenge may be associated with improved health-related quality of life and reduced anxiety. ${ }^{12-14,53}$ Children and their parents may benefit from an oral food challenge as the procedure provides insights into the absence or presence of a food allergy. ${ }^{54}$

In chapter 6 we systematically reviewed the current literature on the impact of diagnostic interventions on generic and disease-specific health-related quality of life. In chapter 2 we investigated the impact of the diagnostic flowchart for peanut 
allergy on patients' and parents' anxiety levels. Finally, in chapter 4 we evaluated the long-term follow-up after a DBPCFC for peanut, including disease-specific health-related quality of life.

\section{Asthma}

Asthma is the most prevalent atopic disease and one of the most prevalent chronic diseases in children, imposing a consistent burden on the health system and on patients. ${ }^{3,55}$ Asthma has a significant detrimental impact on quality of life of children, especially when asthma is uncontrolled. ${ }^{56,57}$ Adequate asthma control is the main management goal in asthma treatment. Numerous studies have shown asthma control to be suboptimal despite the availability of effective asthma treatment. ${ }^{58-64}$ Several predictors of uncontrolled asthma in children have been reported including demographic characteristics (e.g. age, male gender and maternal education level), environmental characteristics (e.g. exposure to indoor smoking and pet ownership), treatment characteristics (e.g. high use of short-acting $\beta 2$-agonists and incorrect inhaler technique) and co-existing atopic diseases (e.g. food allergy and allergic rhinitis). ${ }^{56,65-67}$ We investigated the prevalence and predictors of uncontrolled asthma among children who were referred for asthma or children who were referred for the evaluation of atopic diseases other than asthma to secondary care in chapter 7 .

Uncontrolled asthma, including asthma symptoms and asthma exacerbations, often occur after exposure to allergic (e.g. pollen or animals) or non-allergic (e.g. infections or physical exercise) asthma triggers. Previous research suggests that a higher number of asthma triggers may be associated with impaired disease-specific quality of life in children with severe or difficult-to-treat asthma and in adults. ${ }^{68-70}$ In chapter 8, we examined the single and composite impact of allergic and nonallergic asthma triggers on disease-specific and generic health-related quality of life in children with asthma in secondary and tertiary care. 


\section{Aim of this thesis}

This thesis focuses on improving diagnostics and quality of life in patients with atopic diseases, particularly in patients with peanut allergy and asthma.

We aimed to:

1. Improve diagnostics in patients with atopic diseases by

- evaluating a diagnostic flowchart for peanut allergy based on SIgE to Ara $\mathrm{h} 2$ in children with suspected peanut allergy in secondary and tertiary care in chapter $\mathbf{2}$,

- validating a cutoff level of sIgE to Ara h 2 and developing a diagnostic flowchart in adults with suspected peanut allergy in chapter 3,

- evaluating the long-term follow-up after double-blind placebocontrolled food challenges for peanut in chapter 4,

- and studying risk factors for atopic diseases and recurrent respiratory infections in chapter 5.

2. Improve quality of life in patients with atopic diseases by

- summarizing current literature on the impact of diagnostic testing for food allergy on quality of life in chapter $\mathbf{6}$,

- evaluating the impact of a diagnostic flowchart for peanut allergy on patients' welfare in chapter $\mathbf{2}$,

- evaluating the long-term impact of a double-blind placebo-controlled food challenge for peanut on quality of life in chapter $\mathbf{4}$,

- investigating the prevalence and predictors of uncontrolled asthma in chapter $\mathbf{7}$,

- and assessing the association between asthma triggers and quality of life in chapter 8.

The clinical implications of our findings are discussed in chapter 9. 


\section{References}

1. Cohen S, Dworetzky M, Frick OL. Coca and Cooke on the classification of hypersensitiveness. J Allergy Clin Immunol. 2003;111(1):205-210.

2. Johansson SGO, Bieber T, Dahl R, et al. Revised nomenclature for allergy for global use: Report of the Nomenclature Review Committee of the World Allergy Organization, October 2003. $J$ Allergy Clin Immunol. 2004;113(5):832-836.

3. Asher M, Montefort S, Bjorksten B, et al. Worldwide time trends in the prevalence of symptoms of asthma, allergic rhinoconjunctivitis, and eczema in childhood. Lancet. 2006;368(9537):733743.

4. Jousilahti $\mathrm{P}$, Laatikainen $\mathrm{T}$, Mäkelä $\mathrm{M}$, Vartiainen $\mathrm{E}$. Asthma and respiratory allergy prevalence is still increasing among Finnish young adults. Eur Respir J. 2016;47(3):985-7.

5. Nwaru BI, Hickstein L, Panesar SS, et al. The epidemiology of food allergy in Europe: A systematic review and meta-analysis. Allergy. 2014;69(1):62-75.

6. Gupta RS, Warren CM, Smith BM, et al. The Public Health Impact of Parent-Reported Childhood Food Allergies in the United States. Pediatrics. 2018;142(6):e20181235.

7. Hill DA, Spergel JM. The Atopic March: Critical Evidence and Clinical Relevance. Ann Allergy Asthma Immunol. 2018;120(2):131-137.

8. Gough $\mathrm{H}$, Grabenhenrich L, Reich A, et al. Allergic multimorbidity of asthma, rhinitis and eczema over 20 years in the German birth cohort MAS. Pediatr Allergy Immunol. 2015;26(5):431-437.

9. Zomer-Kooijker K, van Erp FC, Balemans WAF, van Ewijk BE, van der Ent CK. The expert network and electronic portal for children with respiratory and allergic symptoms: Rationale and design. BMC Pediatr. 2013;13(1):9.

10. Ryle J. The Natural History of Disease. 2nd ed. Oxford: Oxford Univeristy Press; 1948.

11. Chauffard A. Medical prognosis: its methods, its evolution, its limitations. Br Med J. 1913;2:286290.

12. Dunngalvin A, Cullinane C, Daly DA, Blok BMJF, Dubois AEJ, Hourihane JOB. Longitudinal validity and responsiveness of the Food Allergy Quality of Life Questionnaire - Parent Form in children $0-12$ years following positive and negative food challenges. Clin Exp Allergy. 2010:476-485.

13. Soller L, Hourihane J, Dunngalvin A. The impact of oral food challenge tests on food allergy health-related quality of life. Allergy. 2014;69(9):1255-1257.

14. Van Der Velde JL, Flokstra-De Blok BMJ, De Groot H, et al. Food allergy-related quality of life after double-blind, placebo-controlled food challenges in adults, adolescents, and children. $J$ Allergy Clin Immunol. 2012;130(5):1136-1143.e2.

15. Nwaru BI, Hickstein L, Panesar SS, Roberts G, Muraro A, Sheikh A. Prevalence of common food allergies in Europe: A systematic review and meta-analysis. Allergy. 2014;69(8):992-1007.

16. Lyons SA, Burney PGJ, Ballmer-Weber BK, et al. Food Allergy in Adults: Substantial Variation in Prevalence and Causative Foods Across Europe. J Allergy Clin Immunol Pract. 2019;7(6):19201928.

17. Soller L, Ben-Shoshan M, Harrington DW, et al. Adjusting for nonresponse bias corrects overestimates of food allergy prevalence. J Allergy Clin Immunol Pract. 2015;3(2):291-293.e2.

18. Muraro A, Werfel T, Hoffmann-Sommergruber K, et al. EAACI Food Allergy and Anaphylaxis Guidelines: Diagnosis and management of food allergy. Allergy. 2014;69(8):1008-1025. 
19. van Erp FC, Knulst AC, Meijer Y, Gabriele C, van der Ent CK. Standardized food challenges are subject to variability in interpretation of clinical symptoms. Clin Transl Allergy. 2014;4(1):43.

20. van Erp FC, Klemans RJB, Meijer $Y$, et al. Using Component-Resolved Diagnostics in the Management of Peanut-Allergic Patients. Curr Treat Options Allergy. 2016:169-180.

21. Masthoff LN, Mattsson L, Zuidmeer-Jongejan L, et al. Sensitization to Cor a 9 and Cor a 14 is highly specific for a hazelnut allergy with objective symptoms in Dutch children and adults. $J$ Allergy Clin Immunol. 2013;132(2):393-399.

22. Hazebrouck S, Guillon B, Paty E, Dreskin SC, Adel-Patient K, Bernard H. Variable IgE crossreactivity between peanut $2 \mathrm{~S}$-albumins: The case for measuring IgE to both Ara $\mathrm{h} 2$ and Ara $\mathrm{h} 6$. Clin Exp Allergy. 2019;49(8):1107-1115.

23. van Erp FC, Knol EF, Pontoppidan B, Meijer $Y$, van der Ent CK, Knulst AC. The IgE and basophil responses to Ara h 2 and Ara h 6 are good predictors of peanut allergy in children. J Allergy Clin Immunol. 2017;139(1):358-360.e8.

24. Kukkonen AK, Pelkonen AS, Makinen-Kiljunen S, Voutilainen $\mathrm{H}$, Makela MJ. Ara h 2 and Ara 6 are the best predictors of severe peanut allergy: a double-blind placebo-controlled study. Allergy. 2015;70(10):1239-1245.

25. Klemans RJB, Knol EF, Bruijnzeel-Koomen CAFM, Knulst AC. The diagnostic accuracy of specific IgE to Ara $\mathrm{h} 6$ in adults is as good as Ara h 2. Allergy. 2014;69(8):1112-1114.

26. Lehmann K, Schweimer K, Reese G, et al. Structure and stability of $2 \mathrm{~S}$ albumin-type peanut allergens: implications for the severity of peanut allergic reactions. Biochem J. 2006;395(3):463472.

27. Zhuang Y, Dreskin SC. Redefining the major peanut allergens. Immunol Res. 2013;55(1-3):125134.

28. Koplin JJ, Perrett KP, Sampson HA. Diagnosing Peanut Allergy with Fewer Oral Food Challenges. J Allergy Clin Immunol Pract. 2019;7(2):375-380.

29. Kunst NR, Lindvik H, Carlsen K-H, Håland G, Jørgensen E, Lødrup Carlsen KC. Cost-effectiveness of diagnostic algorithms for peanut allergy in children. J Allergy Clin Immunol. 2019;143(3):1243-1246.

30. Mullins RJ, Dear KBG, Tang MLK. Time trends in Australian hospital anaphylaxis admissions in 1998-1999 to 2011-2012. J Allergy Clin Immunol. 2015;136(2):367-375.

31. Platts-Mills TAE. The allergy epidemics: 1870-2010. J Allergy Clin Immunol. 2015;136(1):3-13.

32. Turner PJ, Gowland MH, Sharma V, et al. Increase in anaphylaxis-related hospitalizations but no increase in fatalities: An analysis of United Kingdom national anaphylaxis data, 1992-2012. J Allergy Clin Immunol. 2015;135(4):956-963.e1.

33. Mohn CH, Blix HS, Halvorsen JA, Nafstad P, Valberg M, Lagerløv P. Incidence Trends of Atopic Dermatitis in Infancy and Early Childhood in a Nationwide Prescription Registry Study in Norway. JAMA Netw open. 2018;1(7):e184145.

34. Troeger C, Blacker B, Khalil IA, et al. Estimates of the global, regional, and national morbidity, mortality, and aetiologies of lower respiratory infections in 195 countries, 1990-2016: a systematic analysis for the Global Burden of Disease Study 2016. Lancet Infect Dis. 2018;18(11):1191-1210.

35. Wahn U, Von Mutius E. Childhood risk factors for atopy and the importance of early intervention. J Allergy Clin Immunol. 2001;107(4):567-574.

36. Hagerhed-Engman L, Bornehag CG, Sundell J, Åberg N. Day-care attendance and increased risk for respiratory and allergic symptoms in preschool age. Allergy Eur J Allergy Clin Immunol. 2006;61(4):447-453.

37. Toivonen L, Karppinen S, Schuez-Havupalo L, et al. Burden of recurrent respiratory tract 
infections in children: A prospective cohort study. Pediatr Infect Dis J. 2016;35(12):e362-e369.

38. Rovers MM, Numans ME, Langenbach E, Grobbee DE, Verheij TJM, Schilder AGM. Is pacifier use a risk factor for acute otitis media? A dynamic cohort study. Fam Pract. 2008;25(4):233-236.

39. Grammatikos AP. The genetic and environmental basis of atopic diseases. Ann Med. 2008;40(7):482-495.

40. Turner S. Gene-environment interactions-what can these tell us about the relationship between asthma and allergy? Front Pediatr. 2017;5(May):8-14.

41. Patarčić $\mathrm{I}$, Gelemanović $\mathrm{A}$, Kirin $\mathrm{M}$, et al. The role of host genetic factors in respiratory tract infectious diseases: Systematic review, meta-analyses and field synopsis. Sci Rep. 2015;5(October):1-10.

42. Post MWM. Definitions of quality of life: What has happened and how to move on. Top Spinal Cord Inj Rehabil. 2014;20(3):167-

43. Cui W, Zack MM, Zahran HS. Health-related quality of life and asthma among United States adolescents. J Pediatr. 2015;166(2):358-364.

44. Flokstra-de Blok B, Dubois A, Vlieg-Boerstra B, et al. Health-related quality of life of food allergic patients: comparison with the general population and other diseases. Allergy. 2010;65(2):238-244.

45. Becker-Haimes EM, Diaz KI, Haimes BA, Ehrenreich-May J. Anxiety and Atopic Disease: Comorbidity in a Youth Mental Health Setting. Child Psychiatry Hum Dev. 2017;48(4):528-536.

46. Ferro MA, Van Lieshout RJ, Scott JG, Alati R, Mamun AA, Dingle K. Condition-specific associations of symptoms of depression and anxiety in adolescents and young adults with asthma and food allergy. J Asthma. 2016;53(3):282-288.

47. Cummings AJ, Knibb RC, King RM, Lucas JS, Lucas J, Wellcome SH. The psychosocial impact of food allergy and food hypersensitivity in children, adolescents and their families: a review. Allergy. 2010;65(8):933-945.

48. Saleh-Langenberg J, Goossens NJ, Flokstra-De Blok BMJ, et al. Predictors of health-related quality of life of European food-allergic patients. Allergy. 2015;70(6):616-624.

49. Grabenhenrich LB, Dölle S, Moneret-Vautrin A, et al. Anaphylaxis in children and adolescents: The European Anaphylaxis Registry. J Allergy Clin Immunol. 2016;137(4):1128-1137.e1.

50. Blom WM, Michelsen-Huisman AD, van Os-Medendorp $H$, et al. Accidental food allergy reactions: Products and undeclared ingredients. J Allergy Clin Immunol. 2018;142(3):865-875.

51. Primeau $M N$, Kagan $R$, Joseph $L$, et al. The psychological burden of peanut allergy as perceived by adults with peanut allergy and the parents of peanut-allergic children. Clin Exp Allergy. 2000;30(8):1135-1143.

52. Knibb RC, Semper H. Impact of suspected food allergy on emotional distress and family life of parents prior to allergy diagnosis. Pediatr Allergy Immunol. 2013;24(8):798-803.

53. Zijlstra WT, Flinterman AE, Soeters $L$, et al. Parental anxiety before and after food challenges in children with suspected peanut and hazelnut allergy. Pediatr Allergy Immunol. 2010;21:439-445.

54. Nowak-Wegrzyn A, Assa'ad AH, Bahna SL, Bock SA, Sicherer SH, Teuber SS. Work Group report: Oral food challenge testing. J Allergy Clin Immunol. 2009;123(6 SUPPL.).

55. Ferrante G, La Grutta S. The burden of pediatric asthma. Front Pediatr. 2018;6:1-7.

56. Global Initiative for Asthma (GINA). Global Strategy for Asthma Management and Prevention. Available from: https://ginasthma.org (2018). Accessed Aug 8, 2018.

57. Hossny E, Caraballo L, Casale T, El-Gamal Y, Rosenwasser L. Severe asthma and quality of life. World Allergy Organ J. 2017;10(1):1-8.

58. Rabe KF, Adachi M, Lai CKW, et al. Worldwide severity and control of asthma in children and adults: The global asthma insights and reality surveys. J Allergy Clin Immunol. 2004;114(1):40- 
47.

59. Demoly P, Paggiaro P, Plaza V, et al. Prevalence of asthma control among adults in France, Germany, Italy, Spain and the UK. Eur Respir Rev. 2009;18:105-112.

60. Lai CKW, Guia TS De, Kim Y, et al. Asthma control in the Asia-Pacific region: The Asthma Insights and Reality in Asia-Pacific Study. J Allergy Clin Immunol Allergy Clin Immunol. 2003;111:263-268.

61. Mintz M, Gilsenan AW, Bui CL, et al. Assessment of asthma control in primary care. Curr Med Res Opin. 2009;25(10):2523-2531.

62. Price D, Fletcher M, Molen T Van Der. Asthma control and management in 8,000 European patients: the REcognise Asthma and LInk to Symptoms and Experience ( REALISE ) survey. NPJ Prim Care Respir Med. 2014;12(24):14009.

63. Cazzoletti L, Marcon A, Janson C, et al. Asthma control in Europe: A real-world evaluation based on an international population-based study. J Allergy Clin Immunol. 2007;120(6):13601367.

64. Demoly P, Annunziata K, Gubba E, Adamek L. Repeated cross-sectional survey of patientreported asthma control in Europe in the past 5 years. Eur Respir Rev. 2012;21(123):66-74.

65. Oka A, Hirano T, Yamaji Y, et al. Determinants of Incomplete Asthma Control in Patients with Allergic Rhinitis and Asthma. J Allergy Clin Immunol Pract. 2017;5(1):160-164.

66. Sasaki M, Yoshida K, Adachi Y, et al. Factors associated with asthma control in children: Findings from a national Web-based survey. Pediatr Allergy Immunol. 2014;25(8):804-809.

67. Caffarelli C, Garrubba M, Greco C, Mastrorilli C, Dascola CP. Asthma and food allergy in children: Is there a connection or interaction? Front Pediatr. 2016;4(APR):1-7.

68. Chipps BE, Haselkorn T, Rosén K, Mink DR, Trzaskoma BL, Luskin AT. Asthma Exacerbations and Triggers in Children in TENOR: Impact on Quality of Life. J Allergy Clin Immunol Pract. 2018;6(1):169-176.e2.

69. Luskin AT, Chipps BE, Rasouliyan L, Miller DP, Haselkorn T, Dorenbaum A. Impact of Asthma Exacerbations and Asthma Triggers on Asthma-related Quality of Life in Patients with Severe or Difficult-to-Treat Asthma. J Allergy Clin Immunol Pract. 2014;2(5):544-552.

70. Peterson MGE, Gaeta TJ, Birkhahn RH, Fernández JL, Mancuso C a. History of symptom triggers in patients presenting to the emergency department for asthma. J Asthma. 2012;49(6):629-636. 



\section{Chapter 2}

\section{A diagnostic flowchart for pediatric peanut allergy using Ara $\mathrm{h} 2$ is accurate, safe and reduces costs}




\section{Abstract}

\section{Background}

Several studies showed that SIgE to Ara $\mathrm{h} 2$ is a good predictor of peanut allergy and may reduce the need for double-blind placebo-controlled food challenges (DBPCFCs), yet validation and implementation in clinical practice has not been studied.

\section{Objective}

To validate previously published Ara h 2 cutoff levels and implement a diagnostic flowchart to evaluate its 1) diagnostic performance, 2) costs and 3) impact on patients.

\section{Methods}

Physicians of tertiary $(n=1)$ and secondary $(n=3)$ care centers were instructed to use a flowchart in 150 children with suspected peanut allergy based on the Ara $\mathrm{h} 2$ level $\left(\mathrm{kU}_{\mathrm{A}} / \mathrm{L}\right): \leq 0.1$ home introduction, 0.1-5.0 DBPCFC and $\geq 5.0$ open food challenge. The costs of the flowchart were calculated using financial healthcare data and were compared to the costs of a diagnosis according to the guideline. The State-Trait Anxiety Inventory was administered at baseline, after Ara h 2 was communicated and after 6 months.

\section{Results}

The diagnostic flowchart correctly classified $100 \%$ of children with Ara h $2 \leq 0.1$ $\mathrm{kU}_{\mathrm{A}} / \mathrm{L}$ as peanut-tolerant and $89-97 \%$ of children with Ara $\mathrm{h} 2 \geq 5.0 \mathrm{kU}_{\mathrm{A}} / \mathrm{L}$ as peanut-allergic. None of the children with Ara $\mathrm{h} 2 \leq 0.1 \mathrm{kU}_{\mathrm{A}} / \mathrm{L}$ experienced allergic symptoms during (home) introduction. Mean annual costs of the flowchart were $€ 320-€ 636$ per patient lower compared to the guideline. Parental state anxiety was lower after an Ara $\mathrm{h} 2$ result $\leq 0.1 \mathrm{kU}_{\mathrm{A}} / \mathrm{L}$ compared to baseline.

\section{Conclusion}

Ara h 2 cutoff levels are validated in secondary and tertiary care. Furthermore, the diagnostic flowchart is accurate, safe and reduces costs in children. 


\section{Introduction}

Peanut allergy is one of the most common IgE-mediated food allergies, estimated to affect $0.2-2.5 \%$ of children. ${ }^{1,2}$ An accurate diagnosis of peanut allergy is important to adequately counsel allergic children and their parents on the elimination of peanut, the prevention and the treatment of accidental allergic reactions. In children with suspected peanut allergy who prove to be peanuttolerant, exclusion of peanut allergy is important to guide peanut introduction and to prevent unnecessary elimination diets. In addition, an accurate diagnosis of peanut allergy is important to reduce (parental) anxiety and improve health-related quality of life. ${ }^{3,4}$ The oral food challenge (OFC), particularly the double-blind placebo-controlled food challenge (DBPCFC), is currently the gold standard for the diagnosis of peanut allergy according to the EAACI Food Allergy and Anaphylaxis Guidelines. ${ }^{5}$ However, the DBPCFC is difficult in daily practice as the procedure is time-consuming, costly to patients and health services and might be difficult to access. $^{5,6}$ Thus, there is need for improved diagnostic strategies that could accurately predict the DBPCFC result thereby reducing the number of DBPCFCs.

Various studies, including from our own research group, have reported that sIgE to peanut specific component Ara $\mathrm{h} 2$ is useful to distinguish peanut-allergic from peanut-tolerant children and is highly superior to sIgE to peanut extract and other peanut components. ${ }^{7-11}$ When using the cutoff levels of sIgE to Ara h 2 with the highest negative and positive predictive value, $62 \%$ of children could be classified correctly as peanut-tolerant or peanut-allergic. The validation and implementation of scientific research into daily clinical practice is very important to validate previous findings and evaluate diagnostic accuracy, safety, costs and adherence. However, until now no studies have investigated validation of cutoff levels of sIgE to Ara $\mathrm{h} 2$ and the prospective implementation of a diagnostic flowchart based on sIgE to Ara $\mathrm{h} 2$ in daily practice. Thus, it is unknown whether the use of a diagnostic flowchart based on Ara $\mathrm{h} 2$ in children with suspected peanut allergy is accurate and safe, whether it can be used in different clinical settings (secondary and tertiary care) and whether a diagnostic flowchart is beneficial in terms of costs and patients' welfare.

In the current study, we aimed to validate previously published sIgE to Ara $\mathrm{h} 2$ cutoff levels and implement a diagnostic flowchart based on these cutoff levels to evaluate its 1) diagnostic performance, 2) direct and indirect costs and 3) impact on patients (i.e. anxiety levels). 


\section{Methods}

\section{Study population}

We performed a prospective cohort study in one tertiary care center (i.e. university hospital) and three secondary care centers (i.e. general hospitals) in the Netherlands between January 2017 and July 2018. All consecutive children aged 3.5 to 18 years with suspected peanut allergy, seen by a pediatrician or an allergist, were eligible for inclusion. Suspected peanut allergy was based on a clinical history of an allergic reaction to peanut, peanut sensitization (peanut $\operatorname{sigE} \geq 0.35 \mathrm{kU}_{\mathrm{A}} / \mathrm{L}$ or peanut skin prick test mean wheal size $\geq 3 \mathrm{~mm}$ ) or an elimination diet for peanut for more than 1 year. The study was approved by the ethical committee of the University Medical Center Utrecht (nr. 16-1456/C). Parents and children aged 12 years and older provided written informed consent before enrolment in the study.

\section{Study procedures}

Specific IgE to Ara h 2 was determined in all children using the ImmunoCAP method (Thermo Fisher Scientific, Uppsala, Sweden). Physicians were instructed to follow the diagnostic flowchart based on previous found levels of sIgE to Ara $h 2$ to confirm or exclude peanut allergy as depicted in Figure $1{ }^{7}$ Children with an Ara $\mathrm{h} 2$ level $\leq 0.1 \mathrm{kU}_{\mathrm{A}} / \mathrm{L}$ were considered to have a very high probability of being peanuttolerant and were instructed to introduce peanut at home. Home introduction consisted of a 7-day schedule with increasing amounts of peanuts up to $10 \mathrm{grams}$ of whole peanuts on the last day. Children with an Ara $h 2$ level $\geq 5.0 \mathrm{kU}_{\mathrm{A}} / \mathrm{L}$ were considered to have a very high probability of being peanut-allergic. The purpose of further diagnostic testing in these children was to gain insight into the severity of peanut allergy and the threshold of the peanut allergic reaction. Thus, a 1-day open oral food challenge (OFC) instead of a 2-day double-blind placebo-controlled food challenge (DBPCFC) was indicated. Children with an Ara $\mathrm{h} 2$ level between 0.1 and $5.0 \mathrm{kU}_{\mathrm{A}} / \mathrm{L}$ were considered inconclusive and were supposed to undergo a 2-day DBPCFC. The 2-day DBPCFCs and 1-day open OFCs were performed in a clinical setting with equipment for resuscitation. The verum day of the DBPCFC and open OFC consisted of 7 increasing doses of ginger bread containing $1 \mathrm{mg}$ to $2500 \mathrm{mg}$ peanut protein. ${ }^{12}$ If no or inconclusive symptoms occurred on both days of the DBPCFC or on the day of the open OFC, a consecutive open challenge with 10 grams of whole peanuts was performed. All signs and symptoms during the challenge were scored according to the PRACTALL consensus report. ${ }^{13}$ Subsequently, an expert panel of 3 or 4 experienced pediatric allergologists and food allergy experts, blinded for the Ara $\mathrm{h} 2$ level, individually classified children as peanut-allergic or peanut-tolerant and assessed the severity of symptoms and eliciting doses in peanut-allergic children. A food challenge was considered positive when objective or severe long-lasting or repetitive subjective symptoms occurred. 
The severity of symptoms was graded according to the Sampson classification of anaphylaxis as grade 1 to $5 .{ }^{14}$ Any discrepancies between the experts were discussed in a panel discussion meeting until agreement among all experts was reached.

Demographic and clinical information regarding history of peanut allergy and other atopic diseases was obtained by standardized questionnaires. Asthma, allergic rhinitis, atopic dermatitis and other food allergies were defined as having a doctor's diagnosis.

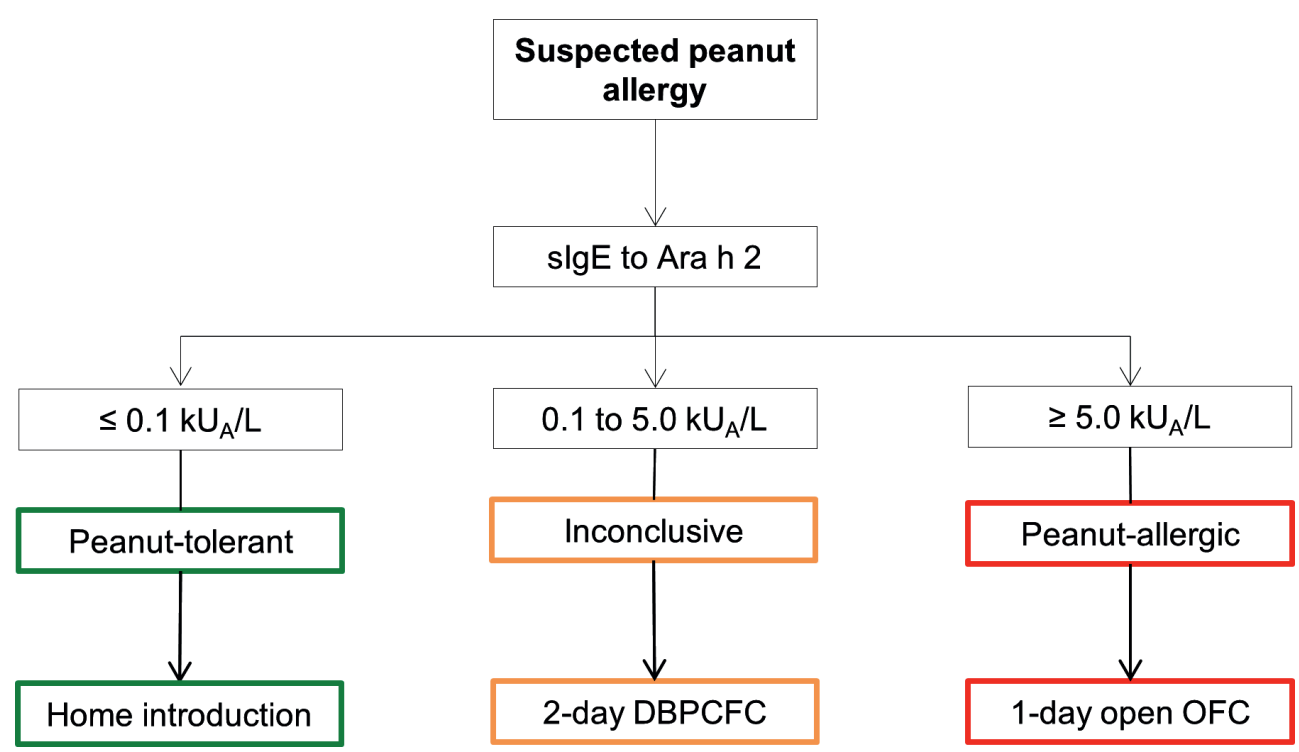

Figure 1. Design of the diagnostic flowchart

Abbreviations: DBPCFC, double-blind placebo-controlled food challenge; OFC, oral food challenge; slgE, specific $\lg E$.

\section{Validation of Ara $\mathbf{h} \mathbf{2}$ cutoff levels}

The validity of the SIgE to Ara $\mathrm{h} 2$ cutoff levels was evaluated by calculating the corresponding sensitivity, specificity, negative predictive value (NPV) and positive predictive value (PPV). Furthermore, the percentage of patients diagnosed correctly using the sIgE to Ara h 2 cutoff levels was calculated.

\section{Diagnostic performance of the diagnostic flowchart}

The diagnostic accuracy of the diagnostic flowchart was evaluated using the area under the curve (AUC) of the receiver operating characteristic. These analyses were stratified by secondary and tertiary care. The safety of the diagnostic flowchart was assessed by calculating the number of allergic reactions during home introduction of peanut. The adherence to the diagnostic flowchart was evaluated by calculating the number of children diagnosed according to the diagnostic flowchart and 
identifying reasons for non-adherence. By exception and with argumentation, physicians could choose a different diagnostic strategy than the diagnostic flowchart based on their expert opinion.

\section{Cost assessment}

The cost assessment was conducted from a societal perspective and included relevant costs borne by the healthcare system (i.e. consultations and diagnostic tests), costs borne by patients and families (i.e. travel expenses) and costs borne by other sectors (i.e. productivity losses) in 12 months after the baseline visit. Costs were based on national guidelines (i.e. Dutch Healthcare Authority and the National Health Care Institute in the Netherlands) and were corrected for inflation using the consumer price index for 2018. ${ }^{15,16}$ The costs were calculated per patient per year by multiplying the volume by the costs per item. The direct medical costs included allergy consultations (i.e. outpatient visits and telephonic consultations) and allergy diagnostic tests (i.e. laboratory tests, skin prick tests, pulmonary function tests and oral food challenges). The data on healthcare use were extracted from the financial healthcare data of the participating centers. Missing data regarding oral food challenges were complemented using electronic patients files. In addition, direct out-of-pocket costs borne by patients and families were included and comprised of travel expenses arising out of the outpatient visits and oral food challenges. Travel expenses were calculated by determining the geographical distance between the families' residence and the hospital using Google Maps and costs for transportation were taken from the national guideline. ${ }^{16}$ The indirect costs comprised of productivity losses due to work absenteeism. The total costs of productivity losses were estimated by multiplying the number of visits to the outpatient clinic and oral food challenges by the time spent for each item, respectively 1 and 8 hours. The lost time of work was then valued according to the Dutch average hourly wage $(€ 38.21) .{ }^{16}$

Mean costs were calculated per patient per year for three scenarios. Scenario A reflected the mean costs of the diagnostic flowchart in daily practice (including children who were diagnosed according to the trajectory of the diagnostic flowchart and children who were diagnosed with other strategies). Scenario B reflected the mean costs of the diagnostic flowchart in theory: only the subset of children who followed the trajectory of the diagnostic flowchart were included in this scenario. Scenario $C$ reflected the mean costs of the diagnostic pathway for peanut allergy according to the current Dutch national guideline. ${ }^{17}$ In scenario $C$, all children with sensitization to peanut (sIgE peanut extract $\geq 0.35 \mathrm{kU}_{\mathrm{A}} / \mathrm{L}$ or skin prick test $\geq 3 \mathrm{~mm}$ ) were supposed to undergo a DBPCFC and all children without peanut sensitization were supposed to start eating peanut at home. The costs for scenario $C$ were derived from the costs in scenario $B$. 


\section{Impact on patients}

Anxiety was measured at baseline, after the telephonic consultation communicating the sIgE to Ara $\mathrm{h} 2$ level and discussing the subsequent diagnostic strategy with parents and patients and after 6 months follow-up. Anxiety was measured using the Spielburger State-Trait Anxiety Inventory (STAI) for parents and the State-Trait Anxiety Inventory for Children (STAIC) for children aged 8 years and older. ${ }^{18,19}$ To assess State-Anxiety (anxiety as an emotional state), parents were instructed to imagine how they would feel if their child was offered peanuts and children to imagine how they would feel if they were offered peanuts. ${ }^{4}$ The items were scored on a 4-point scale with a cumulative score ranging between 20 (low anxiety) and 80 points (high anxiety) for the STAI and between 20 and 60 for the STAIC. The difference in scores was considered clinically relevant when the minimal clinically important difference (MCID) of 10 points was exceeded. ${ }^{20}$

\section{Statistical analysis}

Descriptive statistics were expressed as numbers (percentages) for categorical variables and mean (standard deviation) or median (interquartile range) for continuous variables with a normal or skewed distribution, respectively. Differences in baseline characteristics between children across different sIgE to Ara $\mathrm{h} 2$ levels ( $\leq$ $0.1,0.1-5.0$ and $\geq 5.0 \mathrm{kU}_{\mathrm{A}} / \mathrm{L}$ ) were statistically evaluated by the chi-square test for categorical variables and the one-way analysis of variance (ANOVA) or KruskalWallis test, as appropriate, with correction for multiple testing using the BenjaminiHochberg procedure. Statistical analyses were performed using SPSS for Windows (version 25.0. Armonk, NY: IMB Corp) and R (packages data.table v. 1.12.2 and dplyr v. 0.8.3). A $P$-value of less than 0.05 was considered statistically significant.

\section{Results}

\section{Patient characteristics}

A total of 150 children with suspected peanut allergy were included in secondary care $(65 \%)$ and tertiary care (35\%). Children were grouped based on the level of sIgE to Ara h 2: $\leq 0.1 \mathrm{kU}_{\mathrm{A}} / \mathrm{L}$ in $29(19 \%)$ children, 0.1 to $5.0 \mathrm{kU}_{\mathrm{A}} / \mathrm{L}$ in $68(45 \%)$ children and $\geq 5.0 \mathrm{kU}_{\mathrm{A}} / \mathrm{L}$ in $53(35 \%)$ children. Overall, children had a median (interquartile range) age of 7.9 (5.4 to 13.0) years (Table 1 ).

Children were suspected of peanut allergy because of a previous reaction to peanut (71\%), sensitization without previous ingestion (16\%) or an elimination diet for more than 1 year (13\%). Children with a sIgE to Ara $h 2$ level $\leq 0.1 \mathrm{kU}_{\mathrm{A}} / \mathrm{L}$ were significantly older (median age 12.1 years) compared to children with an Ara h 2 level of 0.1 to $5.0 \mathrm{kU}_{\mathrm{A}} / \mathrm{L}$ or $\geq 5 \mathrm{kU}_{\mathrm{A}} / \mathrm{L}$ (median age 7.6 and 7.1 years, respectively) $(p=0.014)$ and were more often suspected of peanut allergy based on an 
elimination diet for at least 1 year (31\%) compared to children with an Ara h 2 level of 0.1 to 5 or $\geq 5$ ( $12 \%$ and $6 \%$, respectively) ( $p=0.014)$.

Table 1. Baseline characteristics

\begin{tabular}{|c|c|c|c|c|c|}
\hline & \multirow[t]{2}{*}{ All children } & \multicolumn{3}{|c|}{ sIgE to Ara $h 2$ category $\left(\mathrm{kU}_{\mathrm{A}} / \mathrm{L}\right)$} & \multirow{2}{*}{$\begin{array}{l}P \text { - } \\
\text { value }^{+}\end{array}$} \\
\hline & & $\leq 0.1$ & 0.1-5.0 & $\geq \mathbf{5 . 0}$ & \\
\hline Number (\%) & 150 & $29(19)$ & $68(45)$ & $53(35)$ & \\
\hline Age in years, median (IQR) & $7.9(5.4-13)$ & $12.1(8-15.7)$ & $7.6(5.2-11.5)$ & $7.1(5.3-10.5)$ & 0.014 \\
\hline Gender: male & $88(59)$ & $11(38)$ & $44(65)$ & $33(62)$ & 0.090 \\
\hline Setting & & & & & 0.860 \\
\hline Secondary care & $97(65)$ & $20(69)$ & $43(63)$ & $34(64)$ & \\
\hline Tertiary care & $53(35)$ & $9(31)$ & $25(37)$ & $19(36)$ & \\
\hline Suspected peanut allergy & & & & & 0.014 \\
\hline Previous reaction & $106(71)$ & $17(59)$ & $53(78)$ & $36(68)$ & \\
\hline Sensitization & $24(16)$ & $3(10)$ & $7(10)$ & $14(26)$ & \\
\hline Elimination diet $>1$ year & $20(13)$ & $9(31)$ & $8(12)$ & $3(6)$ & \\
\hline Previous reaction $^{\ddagger}$ & & & & & 0.453 \\
\hline No reaction & $44(29)$ & $12(41)$ & $15(22)$ & $17(32)$ & \\
\hline Grade 1 & $31(21)$ & $6(21)$ & $15(22)$ & $10(19)$ & \\
\hline Grade 2 & $32(21)$ & $3(10)$ & $20(29)$ & $9(17)$ & \\
\hline Grade 3 & $18(12)$ & $2(7)$ & $8(12)$ & $8(15)$ & \\
\hline Grade 4 & $21(14)$ & $4(14)$ & $9(13)$ & $8(15)$ & \\
\hline Grade 5 & 0 & 0 & 0 & 0 & \\
\hline Missing data & $4(3)$ & $2(7)$ & $1(1)$ & $1(2)$ & \\
\hline $\begin{array}{l}\text { Elimination other food } \\
\text { allergens }\end{array}$ & & & & & 0.266 \\
\hline No & $42(28)$ & $9(31)$ & $22(32)$ & $11(21)$ & \\
\hline 1 or 2 & $43(29)$ & $5(17)$ & $20(29)$ & $18(34)$ & \\
\hline 3 or 4 & $30(20)$ & $5(17)$ & $17(25)$ & $8(15)$ & \\
\hline 5 or 6 & $27(18)$ & $7(24)$ & $8(12)$ & $12(23)$ & \\
\hline 7 or more & $8(5)$ & $3(10)$ & $1(1)$ & $4(8)$ & \\
\hline \multicolumn{6}{|l|}{ Atopic comorbidities } \\
\hline Atopic dermatitis & 118 (79) & $21(72)$ & $57(84)$ & $40(75)$ & 0.453 \\
\hline Allergic rhinitis & $70(47)$ & $17(59)$ & $37(54)$ & $16(30)$ & 0.042 \\
\hline Asthma & $48(32)$ & $5(17)$ & $22(32)$ & $21(40)$ & 0.207 \\
\hline
\end{tabular}

Value are numbers (\%), unless otherwise indicated.

${ }^{\dagger}$ Benjamini-Hochberg adjusted $P$-values for comparison of differences among 3 groups.

${ }^{\ddagger}$ Missing data $\mathrm{n}=4$. Most severe reaction according to the Sampson classification of anaphylaxis.

Abbreviations: IQR, interquartile range; slgE, specific IgE.

\section{Validation of Ara h $\mathbf{2}$ and diagnostic accuracy and safety of the flowchart}

Figure 2 shows the results of the diagnostic flowchart. All children with a sIgE to Ara $\mathrm{h} 2$ level $\leq 0.1 \mathrm{kU}_{\mathrm{A}} / \mathrm{L}$ were classified as peanut-tolerant after home introduction or diagnostic testing (i.e. food challenge or clinical introduction). Fifty-eight percent of children with a sIgE to Ara $\mathrm{h} 2$ level between 0.1 and $5.0 \mathrm{kU}_{\mathrm{A}} / \mathrm{L}$ were classified as peanut-allergic and $42 \%$ as peanut-tolerant. Eighty-nine percent of children with a SIgE to Ara $h 2$ level $\geq 5 \mathrm{kU}_{\mathrm{A}} / \mathrm{L}$ were classified as peanut-allergic, $3 \%(n=1)$ as peanut-tolerant (Ara h 2 level $7.1 \mathrm{kU}_{\mathrm{A}} / \mathrm{L}$ ) and $8 \%(\mathrm{n}=3)$ as inconclusive. Children with an inconclusive result did not complete the open OFC due to aversion $(n=1)$, 
mild abdominal pain $(n=1)$ or mild abdominal pain and nausea $(n=1)$. Thus, we could classify $100 \%$ of children with a sIgE to Ara h 2 level $\leq 0.1 \mathrm{kU}_{\mathrm{A}} / \mathrm{L}$ correctly as peanut-tolerant (i.e. $100 \%$ negative predictive value). Furthermore, we could classify $89 \%$ of children with a sIgE to Ara $\mathrm{h} 2$ level $\geq 5.0 \mathrm{kU}_{\mathrm{A}} / \mathrm{L}$ correctly as peanut-allergic if all children with an inconclusive outcome would be peanut-tolerant or $97 \%$ of children if all these children would be peanut-allergic (i.e. $89-97 \%$ positive predictive value). The sensitivity, specificity and predictive values of the sIgE to Ara h 2 cutoff levels are depicted in Supplemental Table 1.

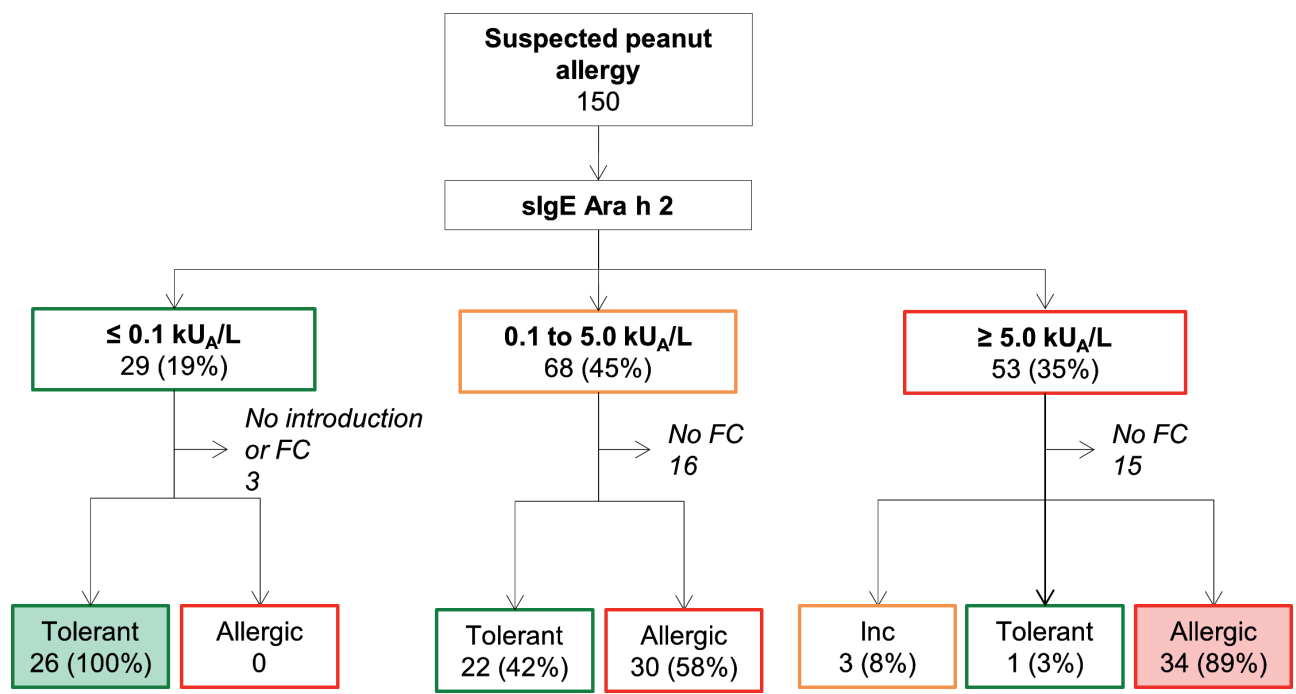

Figure 2. Results of the diagnostic flowchart

Values are numbers (\%).

Abbreviations: FC, food challenge, Inc, inconclusive; sIgE, specific IgE.

Specific IgE to Ara h 2 was a strong predictor of peanut allergy and showed high discriminative capacity (AUC 0.94; 95\% confidence interval [CI] 0.90-0.98) (Figure 3). A stratified analysis by health care line showed high discriminative capacity in children in tertiary care (AUC 0.99; 0.98-1.00) and in secondary care (AUC 0.92; 0.86-0.98). Finally, the diagnostic flowchart was safe as none of the children with an Ara $\mathrm{h} 2$ level $\leq 0.1 \mathrm{kU}_{\mathrm{A}} / \mathrm{L}$ experienced allergic symptoms during peanut introduction. 


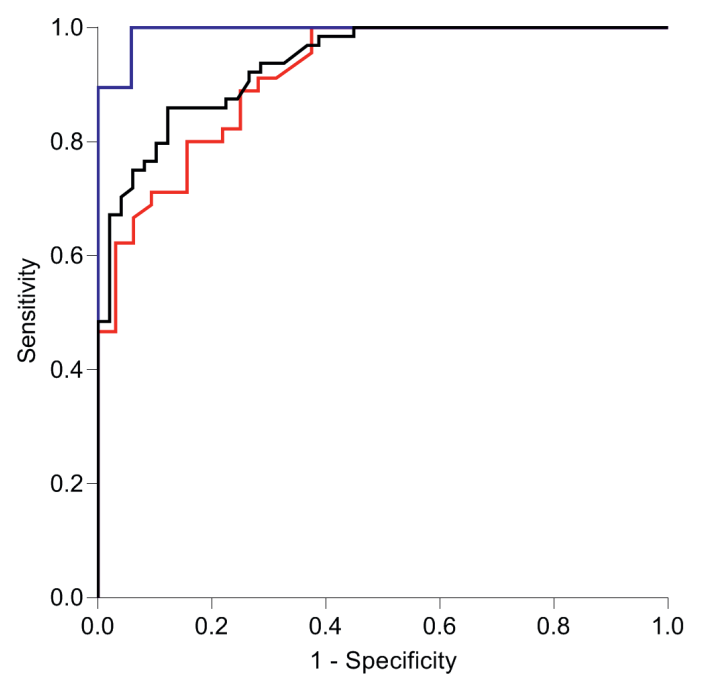

AUC $(95 \% \mathrm{Cl})$ of slgE to Ara h 2

- All 113 children: 0.94 (0.90-0.98)

— 77 children in secondary care: $0.92(0.86-0.98)$

- 36 children in tertiary care: $0.99(0.98-1.00)$

Figure 3. ROC-curves of sIgE to Ara h 2

Abbreviations: $A \cup C$, area under the curve; $\mathrm{Cl}$, confidence interval; $R O C$, receiver operating characteristic; slgE, specific IgE.

\section{Adherence to the diagnostic flowchart}

Overall, 92 of $150(61 \%)$ children followed the trajectory of the diagnostic flowchart. Thirty-four (23\%) children were not further evaluated after Ara h 2 determination and in 24 (16\%) children, a different diagnostic strategy was chosen (Supplemental Figure 1). The most common reason to choose a different diagnostic strategy in children with an Ara $\mathrm{h} 2$ level $\leq 0.1$ and 0.1 to $5.0 \mathrm{kU}_{\mathrm{A}} / \mathrm{L}$ was patients' preference due to anxiety of the child and/or parent(s). However, baseline state anxiety in parents who reported anxiety as reason to choose a different diagnostic strategy was comparable to state anxiety in parents who did not report anxiety (state anxiety 50 and 52 , respectively). The most frequent reason to choose a different diagnostic strategy in children with an Ara $\mathrm{h} 2$ level $\geq 5.0 \mathrm{kU}_{\mathrm{A}} / \mathrm{L}$ was the physician's preference not to perform an oral food challenge due to a suggestive clinical history of previous reaction to peanut and/or anaphylaxis (Supplemental Figure 2).

\section{Cost assessment}

Mean (95\% CI) cumulative costs of the diagnostic flowchart in daily practice (scenario A) and the diagnostic flowchart in theory (scenario B) were $€ 2120$ (€1924 - € 2317) and €2437 (€2189-€2685) per patient per year, respectively (Table 2; Figure 4). Mean costs of the diagnostic flowchart in practice were lower because 34 children did not undergo an oral food challenge. Mean cumulative costs of the diagnostic pathway according to the current national guideline were $€ 2757$ ( $€ 2441$ - $€$ 3072) per patient per year. Thus, the use of the diagnostic flowchart may reduce direct and indirect food-allergy related costs by $€ 320$ (i.e. flowchart in theory) to $€ 636$ (i.e. flowchart in daily practice) per patient per year compared to the current national guideline. 
Table 2. Mean costs per patient per year

\begin{tabular}{|c|c|c|c|}
\hline & & & C \\
\hline & Flowchart in practice & Flowchart in theory & Guideline \\
\hline & Mean $(95 \% \mathrm{CI})$ & Mean $(95 \% \mathrm{CI})$ & Mean $(95 \%$ CI) \\
\hline Number & 150 & 92 & 134 \\
\hline Total costs & $€ 2120$ (1924-2317) & $€ 2437$ (2189- 2685) & $€ 2757$ (2441- 3072) \\
\hline \multicolumn{4}{|l|}{ Direct costs } \\
\hline \multicolumn{4}{|l|}{ Consultations } \\
\hline Outpatient clinic & $€ 319$ (270-369) & $€ 269(227-311)$ & $€ 246(179-314)$ \\
\hline Telephone & $€ 67(58-77)$ & $€ 72(59-84)$ & $€ 62(44-80)$ \\
\hline \multicolumn{4}{|l|}{ Diagnostic testing } \\
\hline Food challenges & $€ 937(809-1,064)$ & $€ 1204(1,041-1,367)$ & $€ 1,433(1,233-1,634)$ \\
\hline Laboratory testing & $€ 112(95-129)$ & $€ 103(81-126)$ & $€ 116(77-155)$ \\
\hline Skin prick testing & $€ 48(34-61)$ & $€ 44(28-60)$ & $€ 47(20-73)$ \\
\hline Lung function & $€ 44(26-61)$ & $€ 26(13-40)$ & $€ 29(5-53)$ \\
\hline Travel expenses & $€ 54(45-63)$ & $€ 54(44-64)$ & $€ 60(44-75)$ \\
\hline \multicolumn{4}{|l|}{ Indirect costs } \\
\hline Productivity loss & $€ 540(477-602)$ & $€ 666(584-47)$ & $€ 763(664-86)$ \\
\hline
\end{tabular}

Including all children (A), children diagnosed according to the diagnostic flowchart (B) and a scenario analysis on the costs for children diagnosed according to the guideline (C).

Abbreviations: $\mathrm{Cl}$, confidence interval.

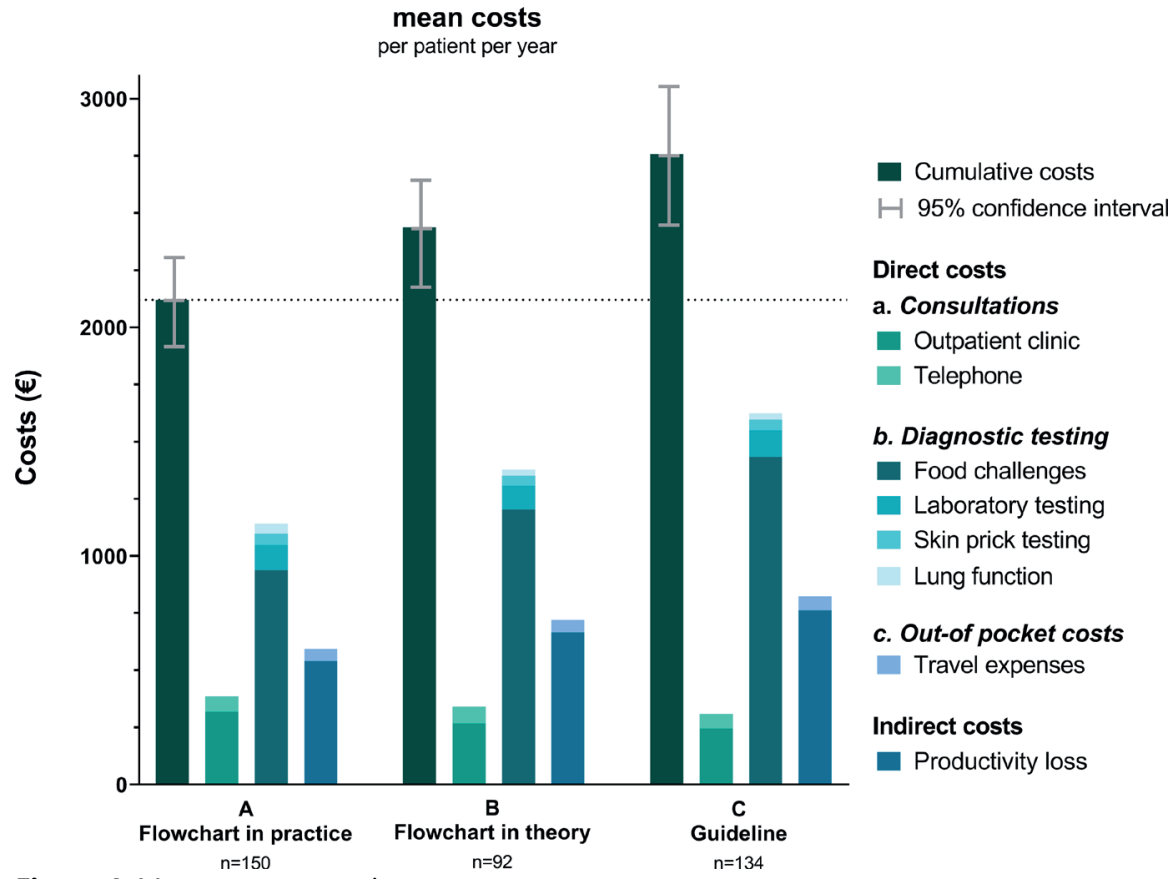

Figure 4. Mean costs per patient per year

Including all children (A), children diagnosed according to the diagnostic flowchart (B) and a scenario analysis on the costs for children diagnosed according to the guideline (C).

Abbreviations: $n$, number. 


\section{Impact on patients}

Parental state anxiety was high at baseline in all Ara $\mathrm{h} 2$ groups with a mean (standard error [SE]) score of 57.7 (2). Parental state anxiety reduced clinically relevant after the telephonic consultation discussing the Ara $\mathrm{h} 2$ result if the Ara $\mathrm{h} 2$ level was $\leq 0.1 \mathrm{kU}_{\mathrm{A}} / \mathrm{L}$ (mean difference, MD 17; SE 4.1) (Figure 5). In parents of children with an Ara $\mathrm{h} 2$ level $>0.1 \mathrm{kU}_{\mathrm{A}} / \mathrm{L}$, state anxiety reduced after 6-months follow-up if the child underwent an oral food challenge (MD 17.9; SE 3.2) and not if the child did not undergo an oral food challenge (MD 5; SE 2.9). Parental state anxiety reduced clinically relevant both after a positive and negative oral food challenge result with a mean (SE) difference of 16.6 (3.6) and 19.6 (6) respectively. Child-reported state anxiety did not improve clinically relevant during follow-up.
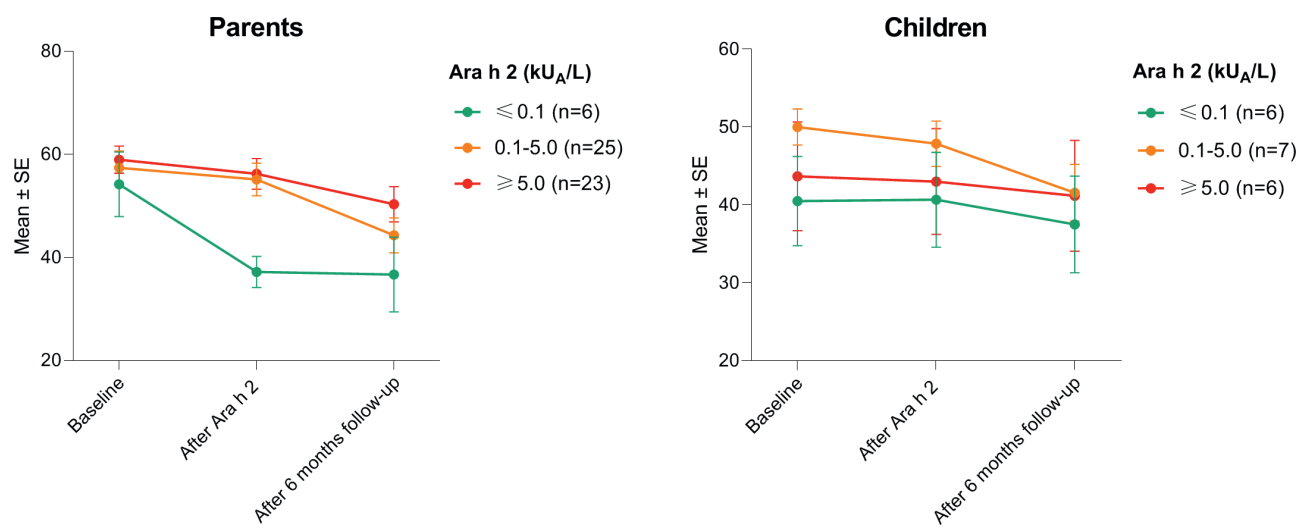

Figure 5. State anxiety of parents and children across groups with different Ara $\mathrm{h} 2$ levels The cumulative score for the state anxiety of parents ranged between 20 (low anxiety) and 80 points (high anxiety) and for state anxiety of children between 20 and 60 .

Abbreviations: $n$, number; SE, standard error.

\section{Discussion}

This is the first study investigating the validation of sIgE to Ara $\mathrm{h} 2$ cutoff levels and the implementation of a diagnostic flowchart based on SIgE to Ara $\mathrm{h} 2$ in children with suspected peanut allergy. Our previously published sIgE to Ara h 2 cutoff levels were validated in children in secondary and tertiary care. ${ }^{7}$ The implemented diagnostic flowchart accurately classified children as peanut-tolerant and peanutallergic with a negative and positive predictive value of $100 \%$ and $89-97 \%$, respectively. Furthermore, the diagnostic flowchart was safe to use in daily clinical practice and could reduce direct and indirect costs of diagnosing peanut allergy by $€ 320$ to $€ 636$ per patient per year compared to diagnosing peanut allergy according to the current national guideline. 
All children in our cohort who were not sensitized to Ara h 2 were peanut-tolerant, even when the child reported a severe allergic reaction to peanut in clinical history. It should be kept in mind that rare cases of (severe) peanut allergy have been described in literature in children who were not sensitized to Ara h $2 .^{21-25}$ These children might be sensitized to other peanut components than Ara $\mathrm{h} 2$, for example Ara h 6 or Ara h $7 .^{26,27}$ However, we believe our diagnostic flowchart is safe to use as the probability of an allergic reaction in children with an Ara $\mathrm{h} 2$ level $\leq 0.1 \mathrm{kU}_{\mathrm{A}} / \mathrm{L}$ is very low ( $0 \%$ in this and in our previous study) and children are instructed to introduce peanut according to an introduction schedule starting at a very small amount of peanut. The adherence to the diagnostic flowchart for children with an Ara $\mathrm{h} 2$ level $\leq 0.1 \mathrm{kU}_{\mathrm{A}} / \mathrm{L}$ was low. The majority of children with an Ara $\mathrm{h} 2$ level $\leq$ $0.1 \mathrm{kU}_{\mathrm{A}} / \mathrm{L}$ in our cohort introduced peanut in the hospital and not at home because children, parents or physicians (unfairly afterwards) feared an allergic reaction to peanut. In this regard, physicians may consider introducing peanut at the outpatient clinic instead of in the hospital to further reduce costs.

We also conclude that total direct and indirect costs seemed to be lower when children were diagnosed according to the diagnostic flowchart compared to the current national guideline. With an estimated 2400 referrals per year in the Netherlands this implicates a reduction in societal costs of $€ 767,101$ per year when all children adhere to the diagnostic flowchart up to $€ 1,526,688$ per year in daily practice. ${ }^{1,28}$ A model-based cost-effectiveness analysis has been performed in a Norwegian cohort of children with suspected peanut allergy. ${ }^{29}$ In support of our findings, the authors reported that a diagnostic flowchart based on peanut components (i.e. Ara h 1, 2, 3, 8 and 9) appeared to be more cost-effective than current clinical practice based on sIgE to peanut extract, skin prick testing and oral food challenges.

We showed that parental state anxiety is largely reduced after a negative Ara $\mathrm{h} 2$ result and after oral food challenges, irrespective of the oral food challenge result. Parental state anxiety remained high during 6-months follow-up if children (were) declined further diagnostic testing after a positive Ara h 2 outcome (i.e. $>0.1$ $\mathrm{kU}_{\mathrm{A}} / \mathrm{L}$ ). One of the main reasons to decline further diagnostic testing was anxiety. These results further underline the beneficial impact of a clear diagnosis of food allergy on parental state anxiety and quality of life as previously reported. ${ }^{3,4}$ Further research is needed to investigate how to handle and/or reduce food-related anxiety in children, especially when anxiety is the reason to decline further diagnostic testing.

To appreciate the results of our study, several limitations should be taken into account. First, our study lacked a control group limiting the ability to compare the diagnostic flowchart to current daily clinical practice according to the guideline. 
However, by comparing the flowchart in theory, in practice and a guidelinescenario we are able to draw valid conclusions regarding the costs of the diagnostic flowchart. Second, we may have underestimated the direct out-of-pocket costs borne by patients and families as we included only the costs of travel to the hospital. A recent systematic review assessed the economic burden of food allergy and showed that food allergy is associated with substantial out-of-pocket costs, for instance the costs of special allergen-free foods, costs of safe child care and copayments for medications. ${ }^{30,31}$ In addition, there is a risk of registration error as direct costs were based on financial healthcare data and indirect costs were based on the average hourly wage. However, our results on the difference in costs between the diagnostic flowchart and the current guideline is not likely influenced by these limitations as the data collection methods were comparable between the analyzed scenarios. Finally, one fourth of children were not included in the analysis on the validation of Ara $\mathrm{h} 2$ cutoff levels and the diagnostic accuracy and safety of the diagnostic flowchart as these children did not undergo further diagnostic testing after the Ara h 2 result. However, the denoted reasons to decline further testing provide meaningful insight into psychosocial barriers and practical implications of the implementation of a new diagnostic strategy in daily practice.

The results of our study are strengthened by the prospective recruitment of all children with a suspected peanut allergy in four centers representing a large area in the Netherlands. In addition, this is the first study that evaluated the validation of Ara $\mathrm{h} 2$ cutoff levels and the prospective implementation of a diagnostic flowchart based on sensitization to peanut component Ara $\mathrm{h} 2$ in daily practices in secondary and tertiary care. Our study assessed a broad range of relevant outcomes, including validation, diagnostic accuracy, safety, adherence, direct and indirect costs and impact on patients.

In conclusion, previously published cutoff levels of sIgE to Ara $\mathrm{h} 2$ were validated in children with suspected peanut allergy in secondary and tertiary care. The diagnostic flowchart based on these Ara h 2 cutoff levels was accurate, safe and beneficial in terms of costs and parental anxiety levels. Our results support the continued use of the diagnostic flowchart and implementation of the diagnostic flowchart in our national guideline. Furthermore, an (adapted) diagnostic flowchart may be used in other countries after international validation studies. 


\section{Acknowledgments}

We thank Eva Koffeman, Ted Klok and Jurgen Jansen for their help with patient inclusion. We thank Joyce Faber, Linda Oudenaller, Lisanne van Berkel and Francina de Graaf for their help with collecting patient data. 


\section{References}

1. Nwaru BI, Hickstein L, Panesar SS, Roberts G, Muraro A, Sheikh A. Prevalence of common food allergies in Europe: A systematic review and meta-analysis. Allergy. 2014;69(8):992-1007.

2. Perkin MR, Logan $K$, Tseng $A$, et al. Randomized Trial of Introduction of Allergenic Foods in Breast-Fed Infants. N Engl J Med. 2016;374(18):1733-1743.

3. Kansen HM, Le TM, Meijer $Y$, et al. The impact of oral food challenges for food allergy on quality of life: A systematic review. Pediatr Allergy Immunol. 2018;29(5):527-537.

4. Zijlstra WT, Flinterman $A E$, Soeters $L$, et al. Parental anxiety before and after food challenges in children with suspected peanut and hazelnut allergy. Pediatr Allergy Immunol. 2010;21:439-445.

5. Muraro A, Werfel T, Hoffmann-Sommergruber K, et al. EAACI Food Allergy and Anaphylaxis Guidelines: Diagnosis and management of food allergy. Allergy. 2014;69(8):1008-1025.

6. van Erp FC, Knulst AC, Meijer Y, Gabriele C, van der Ent CK. Standardized food challenges are subject to variability in interpretation of clinical symptoms. Clin Transl Allergy. 2014;4(1):43.

7. van Erp FC, Knol EF, Pontoppidan B, Meijer Y, van der Ent CK, Knulst AC. The IgE and basophil responses to Ara h 2 and Ara $\mathrm{h} 6$ are good predictors of peanut allergy in children. $J$ Allergy Clin Immunol. 2017;139(1):358-360.e8.

8. Klemans RJB, Otte $D, K n o l ~ M$, et al. The diagnostic value of specific IgE to Ara $\mathrm{h} 2$ to predict peanut allergy in children is comparable to a validated and updated diagnostic prediction model. J Allergy Clin Immunol. 2013;131(1):157-163.

9. Ebisawa $M$, Moverare $R$, Sato $S$, Borres MP, Ito $K$. The predictive relationship between peanutand Ara h 2-specific serum IgE concentrations and peanut allergy. J Allergy Clin Immunol Pr. 2015;3(1):131-2.e1.

10. Suratannon N, Ngamphaiboon J, Wongpiyabovorn J, Puripokai P, Chatchatee P. Componentresolved diagnostics for the evaluation of peanut allergy in a low-prevalence area. Pediatr Allergy Immunol. 2013;24(7):665-670.

11. Eller E, Bindslev-Jensen C. Clinical value of component-resolved diagnostics in peanut-allergic patients. Allergy Eur J Allergy Clin Immunol. 2013;68(2):190-194.

12. Vlieg-Boerstra BJ, Herpertz I, Pasker L, et al. Validation of novel recipes for double-blind, placebo-controlled food challenges in children and adults. Allergy Eur J Allergy Clin Immunol. 2011;66(7):948-954.

13. Sampson HA, Wijk G Van, Bindslev-jensen C, Sicherer S. Standardizing double-blind, placebocontrolled oral food challenges: American Academy of Allergy, Asthma \& Immunology European Academy of Allergy and Clinical Immunology PRACTALL consensus report. J Allergy Clin Immunol. 2012;130(6):1260-1274.

14. Sampson HA. Anaphylaxis and emergency treatment. Pediatrics. 2003;111:1601-1608.

15. Tarieventabel dbc-zorgproducten en overige zorgproducten per 1 januari 2018 - Nederlandse Zorgautoriteit. https://puc.overheid.nl/nza/doc/PUC_13274_22/1/. Accessed December 6, 2019.

16. Hakkaart-van Roijen L, van der Linden N, Bouwmans C, Kanters T, Tan SS. Richtlijn voor het uitvoeren van economische evaluaties in de gezondheidszorg (Bijlage 1). https://www.zorginstituutnederland.nl/over-ons/publicaties/publicatie/2016/02/29/richtlijn-

voor-het-uitvoeren-van-economische-evaluaties-in-de-gezondheidszorg. Published 2015. Accessed December 9, 2019.

17. Nederlandse Vereniging voor Allergologie. Richtlijn Voedselprovocatie. https://www.nvk.nl/Portals/0/richtlijnen/Voedselprovocatie/Richtlijn Voedselprovocatie.pdf. Published 2015. Accessed December 9, 2019.

18. Spielberger C, Gorsuch R, Lushene R, Vagg P, Jacobs G. Manual for the State-Trait Anxiety Inventory. Palo Alto (CA): Consulting Psychologists; 1983.

19. Spielberger C, Gorsuch R, Lushene R, Vagg P, Jacobs G. Manual for the State-Trait Anxiety Inventory for Children. Palo Alto (CA): Consulting Psychologists; 1973.

20. Corsaletti B, Proença M-D, Bisca G, Leite J, Bellinetti L, Pitta F. Minimal important difference for 
anxiety and depression surveys after intervention to increase daily physical activity in smokers. Fisioter e Pesqui. 2014;21(4):359-364.

21. Asarnoj A, Glaumann S, Elfstrom L, et al. Anaphylaxis to peanut in a patient predominantly sensitized to Ara h 6. Int Arch Allergy Immunol. 2012;159(2):209-212.

22. Leo SH, Dean JM, Jung B, Kuzeljevic B, Chan ES. Utility of Ara h 2 sIgE levels to predict peanut allergy in Canadian children. J Allergy Clin Immunol Pr. 2015;3(6):968-969.

23. Zambrano Ibarra G, Fuentes Aparicio V, Infante Herrero S, Blanca M, Zapatero Remon L. Peanut Allergy in Spanish Children: Comparative Profile of Peanut Allergy versus Tolerance. Int Arch Allergy Immunol. 2019;178(4):370-376.

24. Preece K, Bhatia R, Belcher J, et al. The fraction of exhaled nitric oxide improves prediction of clinical allergic reaction to peanut challenge in children. Clin Exp Allergy. 2014;44(3):371-380.

25. Ballmer-Weber BK, Lidholm J, Fernandez-Rivas $M$, et al. IgE recognition patterns in peanut allergy are age dependent: perspectives of the EuroPrevall study. Allergy. 2015;70(4):391-407.

26. Hazebrouck S, Guillon B, Paty E, Dreskin SC, Adel-Patient K, Bernard H. Variable IgE crossreactivity between peanut $2 \mathrm{~S}$-albumins: The case for measuring IgE to both Ara $\mathrm{h} 2$ and Ara $\mathrm{h} 6$. Clin Exp Allergy. 2019;49(8):1107-1115.

27. Blankestijn MA, Otten HG, Suer W, Weimann A, Knol EF, Knulst AC. Specific IgE to peanut $2 \mathrm{~S}$ albumin Ara h 7 has a discriminative ability comparable to Ara h 2 and 6. Clin Exp Allergy. 2018;48(1):60-65.

28. Le T-M, van Hoffen E, Kummeling I, et al. Food allergy in the Netherlands: differences in clinical severity, causative foods, sensitization and DBPCFC between community and outpatients. Clin Transl Allergy. 2015;5:8.

29. Kunst NR, Lindvik H, Carlsen K-H, Håland G, Jørgensen E, Lødrup Carlsen KC. Cost-effectiveness of diagnostic algorithms for peanut allergy in children. I Allergy Clin Immunol. 2019;143(3):1243-1246.

30. Protudjer JLP, Jansson SA, Arnlind $\mathrm{MH}$, et al. Household costs associated with objectively diagnosed allergy to staple foods in children and adolescents. J Allergy Clin Immunol Pract. 2015;3(1):68-75.

31. Bilaver LA, Chadha AS, Doshi P, Dwyer LO, Gupta RS. Economic burden of food allergy A systematic review. Ann Allergy, Asthma Immunol. 2019;122(4):373-380.e1. 


\section{Supplemental figures}

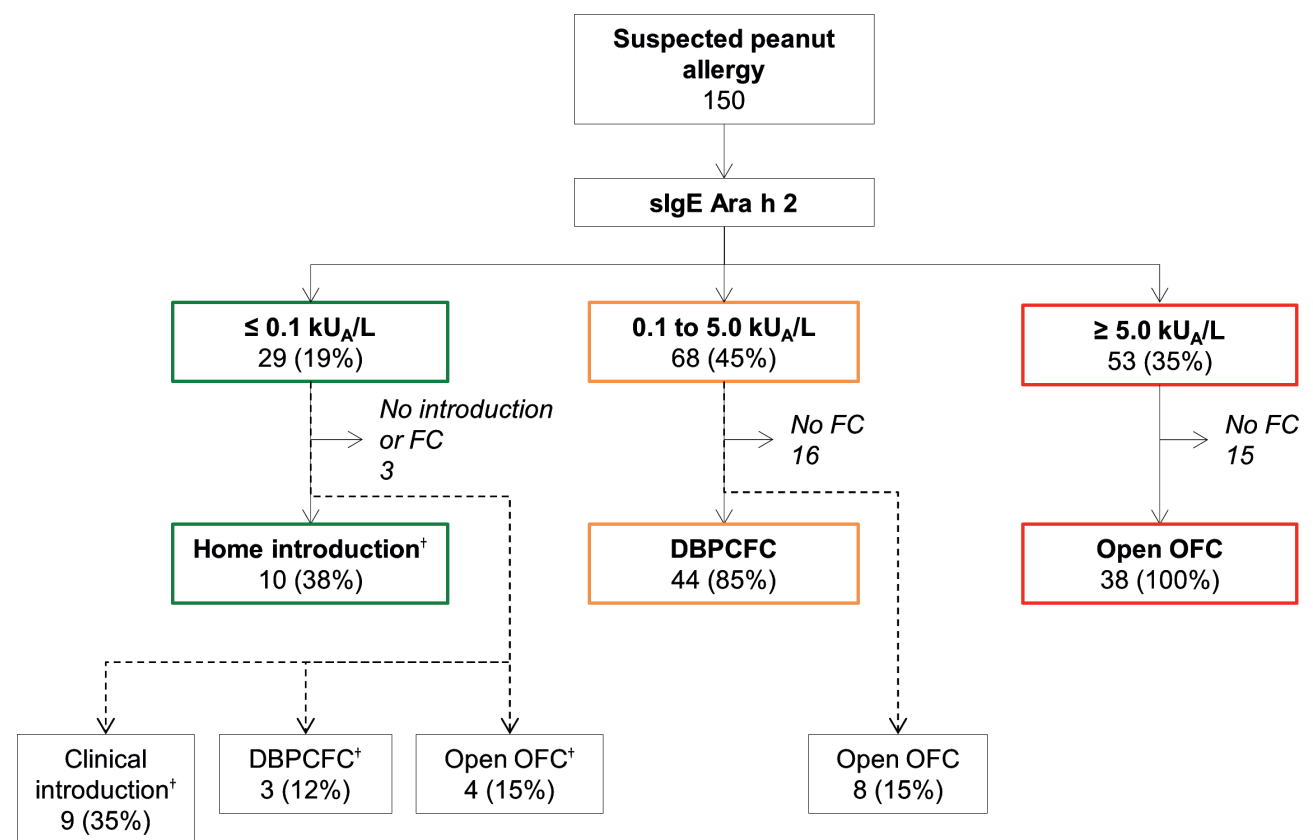

Supplemental Figure 1. Diagnostic pathway of included children

${ }^{\dagger}$ All children were peanut-tolerant.

Abbreviations: $D B P C F C$, double-blind placebo-controlled food challenge; FC, food challenge; OFC, oral food challenge.
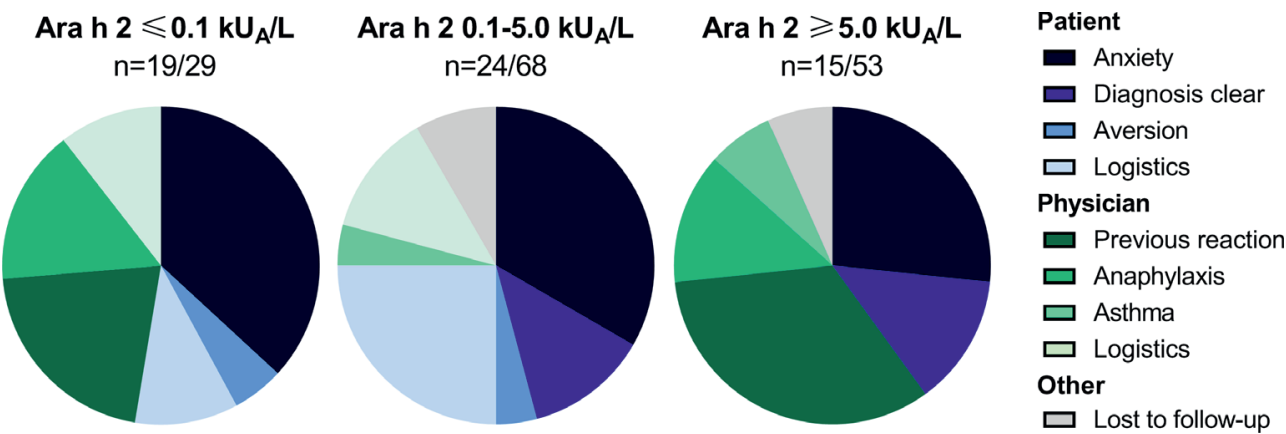

Supplemental Figure 2. Reasons to choose a diagnostic strategy other than the diagnostic flowchart per Ara h 2 category 


\section{Supplemental tables}

Supplemental Table 1. Diagnostic value of sIgE to Ara h 2 cutoff levels to predict peanut allergy

\begin{tabular}{lllllll}
\hline Patients & $\mathbf{N}$ & $\begin{array}{l}\text { Cutoff level } \\
\mathrm{kU}_{\mathrm{A}} / \mathrm{L}\end{array}$ & $\begin{array}{l}\text { Sensitivity } \\
\%\end{array}$ & $\begin{array}{l}\text { Specificity } \\
\%\end{array}$ & $\begin{array}{l}\text { PPV } \\
\%\end{array}$ & $\begin{array}{l}\mathbf{N P V} \\
\%\end{array}$ \\
\hline All children & 113 & 0.1 & 100 & 53 & 74 & 100 \\
& & 5.0 & 53 & 98 & 97 & 62 \\
Secondary care & 77 & 0.1 & 100 & 59 & 78 & 100 \\
& & 5.0 & 49 & 97 & 96 & 57 \\
Tertiary care & \multirow{2}{*}{36} & 0.1 & 100 & 41 & 66 & 100 \\
& & 5.0 & 63 & 100 & 100 & 71 \\
\hline
\end{tabular}

Abbreviations: $n$, number; NPV, negative predictive value; PPV, positive predictive value. 


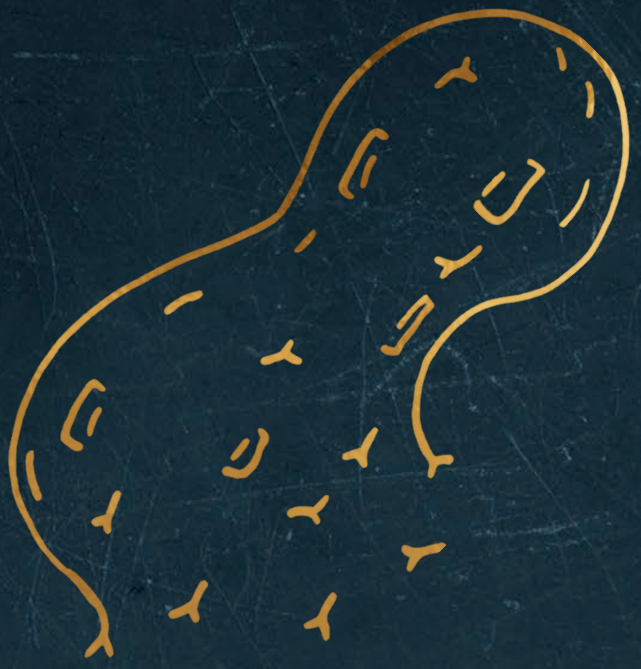

$+1$

$r$ 


\section{Chapter 3}

\section{Accurate prediction of peanut allergy in one third of adults using a validated Ara h 2 cutoff}




\section{Abstract}

\section{Background}

The diagnostic value of peanut components is extensively studied in children, but to a lesser extent in adults with suspected peanut allergy. The use of peanut components in daily practice may reduce the need for double-blind placebocontrolled food challenges (DBPCFCs), however validation studies are currently lacking.

\section{Objective}

To 1) evaluate the diagnostic value of (combined) peanut components and 2) validate a previous found Ara $\mathrm{h} 2$ cutoff level with $100 \%$ positive predictive value (PPV) in adults with suspected peanut allergy.

\section{Methods}

Adults who underwent a peanut DBPCFC were included: 84 patients from a previous study (2002 to 2012) and 70 new patients (2012 to 2019). sIgE to peanut extract, Ara h 1, 2, 3, 6 and 8 was measured using ImmunoCAP. Diagnostic value was assessed with an area under the curve (AUC) analysis.

\section{Results}

In total, 95 (62\%) patients were peanut-allergic. sIgE to Ara h 2 and Ara h 6 were the best predictors with an AUC (95\% CI) of 0.85 (0.79-0.91) and 0.85 (0.79-0.92), respectively. The Ara $\mathrm{h} 2$ cutoff level with $100 \%$ PPV $\left(\geq 1.75 \mathrm{kU}_{\mathrm{A}} / \mathrm{L}\right)$ was validated in the 70 new patients. Thirty percent of all included patients could be classified correctly as peanut-allergic using this validated cutoff level.

\section{Conclusion}

Specific IgE to Ara $\mathrm{h} 2$ and Ara $\mathrm{h} 6$ have equally high discriminative ability. Peanut allergy can be predicted accurately in one third of adults using a validated cutoff level of sIgE to Ara h 2. 


\section{Introduction}

Peanut allergy affects up to $0.60 \%$ of adults in Europe. ${ }^{1-3}$ The reference standard to diagnose or exclude peanut allergy is a double-blind placebo-controlled food challenge (DBPCFC). ${ }^{4}$ However, a DBPCFC is time-consuming, labor-intensive, costly and not without risks because of the potential for severe allergic reactions including anaphylaxis. ${ }^{5}$ Given these limitations, new diagnostic strategies have been evaluated that could reduce the number of DBPCFCs. ${ }^{6}$

Serology tests may be used to reduce the number of DBPCFCs when validated cutoff levels are used. $^{7-9}$ Sensitization to peanut component allergens (e.g. component-resolved diagnostics) has emerged as a useful tool to diagnose peanut allergy with increased diagnostic accuracy compared to sensitization to crude peanut extract or skin prick testing. ${ }^{8,10-16}$ Currently, 17 peanut allergens have been described by the World health Organization and International Union of Immunological Societies (WHO/IUIS) Allergen Nomenclature Sub-committee. ${ }^{17}$ Ara h 2 and Ara h 6 are seed storage proteins and 2S albumins with highly similar protein structure ${ }^{18}$, and have been recognized as the most important allergens associated with peanut allergy. ${ }^{8,10,15}$ Ara $h 7$ is a third $2 S$ albumin and is gaining attention as predictor for peanut allergy. ${ }^{11}$ Ara $h 1$ and Ara $h 3$ are also seed storage proteins and, together with Ara $\mathrm{h} 2$, have been designated as the major peanut allergens. ${ }^{19,20}$ Ara $\mathrm{h} 8$ is a Bet $v 1$ homologous pathogenesis-related (PR)-10 protein which cross-sensitizes with major-birch pollen allergen Bet $v 1$ in adults. ${ }^{21}$ Isolated sensitization to Ara $\mathrm{h} 8$ is associated with mild or no symptoms, although the role of Ara $\mathrm{h} 8$ in multiple sensitized patients remains unclear. ${ }^{22}$ Finally, Ara $\mathrm{h} 9$ is a lipid transfer protein, and a major allergen in the Mediterranean area. ${ }^{23}$

Current evidence on cutoff levels for peanut components in adults is limited, as the majority of research has been conducted in children. Furthermore, validation studies of the diagnostic value of peanut components are currently lacking. Validation studies are warranted to implement research findings into daily practice. A previous study performed in our center included 84 adults, and showed that peanut allergy can be predicted with $100 \%$ positive predictive value (PPV) using a SIgE to Ara $\mathrm{h} 2$ cutoff level of $\geq 1.75 \mathrm{kU}_{\mathrm{A}} / \mathrm{L}$. ${ }^{16}$ Other recent studies have shown that the discriminative ability of sIgE to Ara $h 6$ is comparable to sIgE to Ara $h 2^{8,15,24}$ Peanut component Ara $\mathrm{h} 6$ has mainly been studied using a multiplex assay as opposed to a singleplex assay. ${ }^{14,15,24,25}$ The results of a multiplex assay are semiquantitative and therefore, these results cannot be used to determine cutoff levels. $^{26}$ 
In the current study, we aimed to evaluate the diagnostic value of sIgE to peanut extract, Ara h 1, 2, 3, 6 and 8, and combinations, using a singleplex assay in adults with suspected peanut allergy. Furthermore, we aimed to validate the previously published cutoff level of sIgE to Ara h 2 with $100 \%$ positive predictive value (i.e. $\geq$ $\left.1.75 \mathrm{kU}_{\mathrm{A}} / \mathrm{L}\right)$.

\section{Methods}

\section{Study population}

All 84 adult patients who participated in the previous diagnostic study and underwent a double-blind placebo-controlled food challenge (DBPCFC) for peanut at the University Medical Centre Utrecht between 2002 and June 2012 were eligible for inclusion. ${ }^{16}$ Furthermore, we expanded the cohort with patients who underwent a DBPCFC for peanut between June 2012 and May $2019(n=120)$. The DBPCFC was performed because of a suspected peanut allergy based on clinical history or sensitization. Patients with an inconclusive DBPCFC result $(n=18)$ or without leftover serum $(n=32)$ were excluded from the analysis. Included and excluded patients were comparable in age, gender, presence of allergic rhinitis, severity of peanut allergy and levels of sIgE to peanut extract and components (Supplemental Table 1). The current study was approved by the ethical committee of the University Medical Center Utrecht (no. 18-428).

\section{Oral food challenges}

DBPCFCs were performed in a clinical setting equipped for resuscitation in accordance with the international consensus protocol as previously described. ${ }^{27-29}$ The DBPCFC result was discussed among food allergy experts and was considered positive when objective symptoms occurred ('positive objective'), or when subjective symptoms lasted for at least 45 minutes, or occurred to at least 3 subsequent doses ('positive subjective'). The severity of the DBPCFC was based on the adapted Mueller score: 0 . oral symptoms, 1 . cutaneous symptoms, 2. gastrointestinal symptoms, 3. respiratory symptoms and 4 . cardiovascular symptoms. ${ }^{16,30}$

\section{Sensitization (sIgE) measurements}

Specific IgE (sIgE) values to peanut extract and peanut components Ara h 1, 2, 3, 6 and 8 were determined using the ImmunoCAP platform (Thermo Fisher Scientific Uppsala, Sweden). In the previous diagnostic study, sIgE to peanut extract, Ara h 1 , 2,3 and 8 was already measured in all patients $(n=84)$. In these patients Ara $h 6$ was determined as part of the current study in those with available leftover serum $(n=48)$. In all newly included $(n=70)$ patients, we determined Ara h 1, 2, 3, 6 and 8 . 
In peanut-allergic patients without sensitization to peanut components or peanut extract as measured using the ImmunoCAP platform, sensitization to Ara $h 1,2,3$, $5,6,7,8,9,10,11,14$ and 15 was assessed using a line blot (EUROLINE, EUROIMMUN, Lübeck, Germany) as described elsewhere. ${ }^{11}$

\section{Statistical analyses}

The differences between patients with a positive and a negative DBPCFC result were analyzed using the chi-square test and Mann-Whitney $U$ test, when appropriate. The diagnostic value of sIgE to peanut extract and peanut components was assessed by the area under the curve (AUC) of the receiver-operating characteristic (ROC). The AUC values were compared using DeLong's test for correlated ROC curves. ${ }^{31}$ To evaluate the diagnostic value of all serology tests combined (i.e. sIgE to peanut extract, Ara h 1, 2, 3, 6 and 8), logistic least absolute shrinkage and selection operator (LASSO) regression analysis was performed. The LASSO regression method is based on the regular least-squares method, but includes an additional parameter $\lambda$ to prevent overfitting and automatically deletes unnecessary covariates. ${ }^{32}$ We used cross-validation to select $\lambda$.

The previously published cutoff level of sIgE to Ara h 2 with 100\% positive predictive value (PPV) was validated by calculating the PPV of this cutoff level $(\geq$ $1.75 \mathrm{kU}_{\mathrm{A}} / \mathrm{L}$ ) in the newly included patients (43 peanut-allergic and 27 peanuttolerant patients). The sample size needed to have $95 \%$ confidence and $80 \%$ power to detect a difference of $5 \%$ from a presumed PPV of $98 \%$ was 31 cases. $^{33}$ In addition, the cutoff levels with optimal positive and negative predictive values with corresponding sensitivities and specificities were calculated for peanut extract and all peanut components. Statistical analyses were performed in SPPS for Windows (version 25.0. Armonk, NY: IMB Corp) and R (pROC package v 1.15.3, randomForest package v 4.6-14, OptimalCutpoints package v 1.1-4).

\section{Results}

In total, 154 adults were included after a positive $(n=95)$ or a negative $(n=59)$ DBPCFC result. A positive DBPCFC result was based on objective symptoms in 69 (73\%) patients and on subjective symptoms in 26 (27\%) patients. Patients with a positive DBPCFC result were significantly younger compared to patients with a negative DBPCFC result (median age 25 and 34 years, respectively) and had higher levels of sIgE to peanut extract, Ara h 1,2, 3 and 6 (Table 1). The level of sIgE to Ara h 8 was comparable between patients with a positive and a negative DBPCFC result. 
Table 1. Characteristics of participating patients and details of DBPCFCs

\begin{tabular}{|c|c|c|c|c|}
\hline & Total & $\begin{array}{l}\text { Positive } \\
\text { DBPCFC result }\end{array}$ & $\begin{array}{l}\text { Negative } \\
\text { DBPCFC result }\end{array}$ & $\begin{array}{l}P \text { - } \\
\text { value }\end{array}$ \\
\hline$n(\%)$ & 154 & $95(62)$ & $59(38)$ & \\
\hline Age, median (IQR) & $27(22-38)$ & $25(21-31)$ & $34(24-43)$ & $<0.001$ \\
\hline Male gender & $52(34)$ & $35(37)$ & $17(29)$ & 0.306 \\
\hline Allergic rhinitis $^{+}$ & $69(45)$ & $30(32)$ & $22(37)$ & 0.236 \\
\hline \multicolumn{5}{|l|}{ Sensitization, median (IQR) } \\
\hline $\operatorname{sIg} \mathrm{E}$ peanut extract & $1.65(0.35-11.05)$ & $6.20(0.72-23.70)$ & $0.44(0.16-1.65)$ & $<0.001$ \\
\hline sIgE Ara h 1 & $0.02(0.00-0.61)$ & $0.10(0.01-7.58)$ & $0.00(0.00-0.03)$ & $<0.001$ \\
\hline sIgE Ara h 2 & $0.16(0.03-3.50)$ & $2.20(1.14-10.00)$ & $0.03(0.00-0.08)$ & $<0.001$ \\
\hline sIgE Ara h 3 & $0.03(0.01-0.18)$ & $0.07(0.01-0.92)$ & $0.01(0.00-0.03)$ & $<0.001$ \\
\hline sIgE Ara h $6^{\ddagger}$ & $0.07(0.00-3.14)$ & $1.37(0.08-9.50)$ & $0.00(0.00-0.03)$ & $<0.001$ \\
\hline sIgE Ara h 8 & $2.02(0.11-8.57)$ & $2.05(0.24-8.70)$ & $1.50(0.03-8.53)$ & 0.198 \\
\hline DBPCFC period & & & & 0.952 \\
\hline 2003-2012 & $84(55)$ & $52(55)$ & $32(54)$ & \\
\hline $2012-2019$ & $70(45)$ & $43(45)$ & $27(46)$ & \\
\hline DBPCFC result & & & & NA \\
\hline Positive objective & $69(45)$ & $69(73)$ & NA & \\
\hline Positive subjective & $26(17)$ & $26(27)$ & NA & \\
\hline Negative & $59(38)$ & NA & $59(100)$ & \\
\hline Severity positive DBPCFC ${ }^{\S}$ & & & & NA \\
\hline Mueller 0 & $15(16)$ & $15(16)$ & NA & \\
\hline Mueller 1 & $16(17)$ & $16(17)$ & NA & \\
\hline Mueller 2 & $32(34)$ & $32(34)$ & NA & \\
\hline Mueller 3 & $28(29)$ & $28(29)$ & NA & \\
\hline Mueller 4 & $2(2)$ & $2(2)$ & NA & \\
\hline
\end{tabular}

Value are $n(\%)$ unless otherwise indicated.

${ }^{+}$Missing data $\mathrm{n}=85$

${ }^{\ddagger}$ Missing data $\mathrm{n}=36$

${ }^{\S}$ Missing data $n=1$. Severity of symptoms during the oral food challenge based on the Mueller score with 0 . oral symptoms, 1 . cutaneous symptoms, 2. gastrointestinal symptoms, 3 . respiratory symptoms and 4. cardiovascular symptoms. ${ }^{16,30}$

Abbreviations: DBPCFC, double-blind placebo-controlled food challenge; IQR, interquartile range; NA, not applicable; slgE, specific IgE.

\section{Specific IgE to Ara $\mathbf{h} \mathbf{2}$ and Ara $\mathbf{h} \mathbf{6}$ are the best predictors of peanut allergy}

The levels of sIgE to peanut extract, Ara h 1, 2, 3 and 8 were available in all 154 patients and the level of sIgE to Ara $\mathrm{h} 6$ in 118 patients. The diagnostic value of sIgE to peanut extract, Ara h 1, 2, 3 and 8 were comparable in the cohort of 118 and 154 patients (Supplemental Table 2).

The discriminative abilities of sIgE to Ara h 2 and sIgE to Ara h 6 for predicting peanut allergy were high and comparable, with an AUC (95\% CI) of 0.85 (0.79-0.91) and 0.85 (0.79-0.92), respectively (Figure 1a). The AUC values of sIgE to Ara $h 2$ and Ara $\mathrm{h} 6$ were significantly higher than the AUC values of sIgE to peanut extract, Ara h 1, Ara h 3 and Ara h 8 (Supplemental Table 3). When serology tests were used to 
predict peanut allergy with objective symptoms, the AUC values of sIgE to Ara $\mathrm{h} 2$ and Ara h 6 increased slightly to respectively 0.90 (0.85-0.95) and 0.90 (0.84-0.96) (Figure 1b). The concentrations of sIgE to Ara h 2 and Ara h 6 were strongly correlated (Pearson Correlation coefficient 0.83 ).

The final covariates included in the LASSO model for predicting peanut allergy were sIgE to Ara h 1 and Ara h 6, with Ara h 6 as the most influential predictor. The AUCvalue of the LASSO model was 0.85 (0.78-0.92).

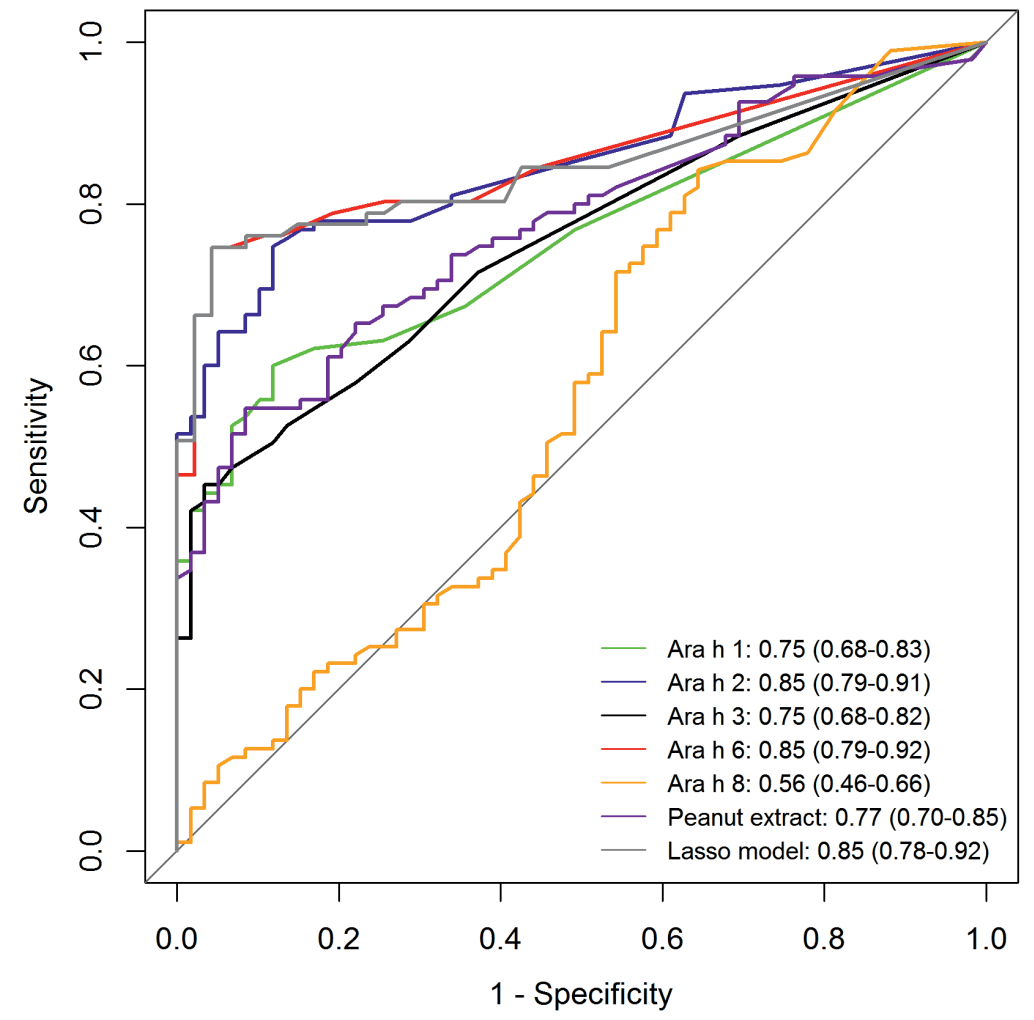

Figure 1a. ROC-curves of serology tests to predict a positive DBPCFC result The Lasso model included sIgE to Ara h 1 and Ara h 6.

Data are AUC values with 95\% confidence interval.

Abbreviations: AUC, area under the curve; DBPCFC, double-blind placebo-controlled food challenge; ROC, receiver-operating characteristic. 


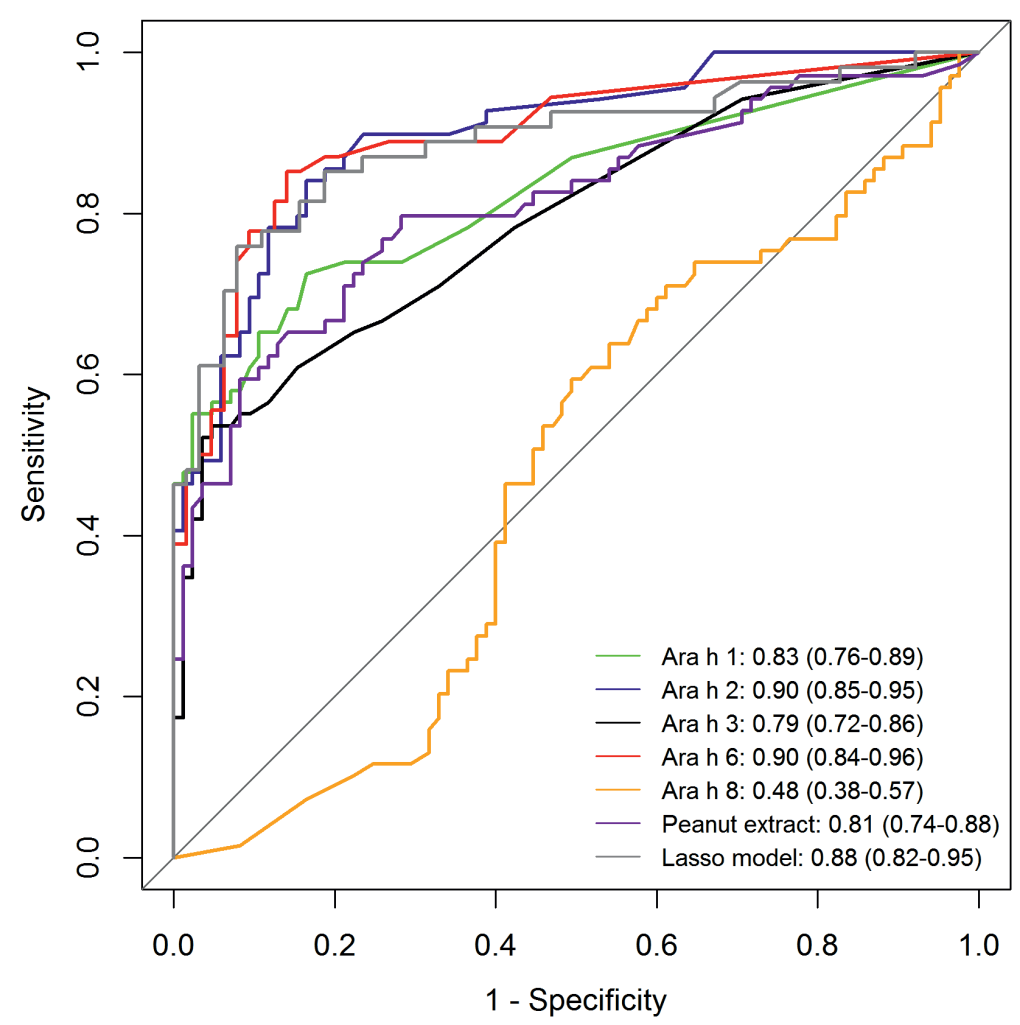

Figure 1b. ROC-curves of serology tests to predict positive DBPCFC result with objective symptoms The Lasso model included sIgE to peanut extract, Ara h 1, Ara h 6 and Ara h 8.

Data are AUC values with $95 \%$ confidence interval.

Abbreviations: AUC, area under the curve; DBPCFC, double-blind placebo-controlled food challenge; $n$, number; $R O C$, receiver-operating characteristic.

\section{The Ara $\mathbf{h} \mathbf{2}$ cutoff level with $\mathbf{1 0 0 \%}$ positive predictive value is validated}

A previous study performed in our center, including 84 adults, showed that peanut allergy can be diagnosed in $28 \%$ of patients by using a sIgE to Ara h 2 cutoff level with $100 \%$ positive predictive value (PPV) of $\geq 1.75 \mathrm{kU}_{\mathrm{A}} / \mathrm{L}^{16}$ In the expanded cohort, including 84 adults from the previous study and 70 adults who were challenged after the first study, this cutoff level has again 100\% positive predictive value (Table 2). Thus, we were able to confirm the previously published cutoff level of Ara $\mathrm{h} 2$ in a larger group of adult patients. Thirty-two percent of patients had an Ara $\mathrm{h} 2$ value above the validated cutoff level and could be predicted correctly as peanut-allergic. Eighty-eight percent of patients above the Ara h 2 cutoff level had a positive DBPCFC result with objective symptoms. The optimal Ara h 2 cutoff level with $100 \%$ PPV in patients who were not part of the previous diagnostic study $(\mathrm{n}=70)$ was $\geq 0.43 \mathrm{kU}_{\mathrm{A}} / \mathrm{L}$. Six patients had an Ara $\mathrm{h} 2$ level between $0.43 \mathrm{kU}_{\mathrm{A}} / \mathrm{L}$ and $1.74 \mathrm{kU}_{\mathrm{A}} / \mathrm{L}$ and were all peanut-allergic (4 with objective symptoms and 2 with subjective symptoms). 


\section{Cutoff levels for Ara $\mathbf{h} \mathbf{6}$ and other peanut components}

The Ara $\mathrm{h} 6$ cutoff level with $100 \%$ PPV was $\geq 1.80 \mathrm{kU}_{\mathrm{A}} / \mathrm{L}$, comparable to the Ara $\mathrm{h} 2$ cutoff level (Table 2). Twenty-eight percent of patients had an Ara $\mathrm{h} 6$ value above this cutoff level and could be predicted correctly as peanut-allergic. The highest NPVs were observed for Ara $\mathrm{h} 6$ and Ara $\mathrm{h} 8$ at a cutoff of $0.1 \mathrm{kU}_{\mathrm{A}} / \mathrm{L}$ and were $81 \%$ and $88 \%$, respectively. The NPVs for sIgE to peanut extract, Ara h 1 , Ara h 2 and Ara h 3 (at a cut off of $0.1 \mathrm{kU}_{\mathrm{A}} / \mathrm{L}$ ) were $78 \%, 58 \%, 79 \%$ and $62 \%$, respectively.

Table 2. Validation of sIgE to Ara h 2 cutoff level and cutoff levels with optimal positive and negative predictive values

\begin{tabular}{lcccccc}
\hline & n & $\begin{array}{c}\text { Cutoff } \\
\text { kUA/L }\end{array}$ & $\begin{array}{c}\text { PPV } \\
\%\end{array}$ & $\begin{array}{c}\text { NPV } \\
\%\end{array}$ & $\begin{array}{c}\text { Sens } \\
\%\end{array}$ & $\begin{array}{c}\text { Spec } \\
\%\end{array}$ \\
\hline $\begin{array}{l}\text { Validation } \\
\text { sIgE to Ara h 2 }\end{array}$ & 70 & 1.75 & 100 & 60 & 58 & 100 \\
Optimal PPV cutoff levels & & & & & & \\
sIgE to Ara h 2 & 154 & 1.75 & 100 & 56 & 52 & 100 \\
sIgE to Ara h 6 & 118 & 1.80 & 100 & 55 & 46 & 100 \\
sIgE to Ara h 1 & 154 & 2.47 & 100 & 49 & 36 & 100 \\
sIgE to Ara h 3 & 154 & 0.88 & 100 & 46 & 26 & 100 \\
sIgE to Ara h 8 & 154 & 100 & 100 & 39 & 1 & 100 \\
sIgE to peanut extract & 154 & 15.10 & 100 & 48 & 34 & 100 \\
Optimal NPV cutoff levels & & & & & & \\
sIgE to Ara h 2 & 154 & 0.02 & 71 & 79 & 94 & 37 \\
sIgE to Ara h 6 & 118 & 0.04 & 88 & 81 & 88 & 81 \\
sIgE to Ara h 1 & 154 & 0.05 & 89 & 58 & 60 & 88 \\
sIgE to Ara h 3 & 154 & 0.01 & 67 & 62 & 88 & 31 \\
sIgE to Ara h 8 & 154 & 0.01 & 64 & 88 & 99 & 12 \\
sIgE to peanut extract & 154 & 0.11 & 67 & 78 & 96 & 24 \\
\hline Abbrevations: $n$
\end{tabular}

Abbreviations: $n$, number of patients included; NPV, negative predictive value; $P P V$, positive predictive value; Sens, sensitivity; slgE, specific IgE; Spec, specificity. 


\section{Higher titers in patients with objective symptoms}

Figure 2 shows the percentage of patients with and without sensitization (cutoff $0.10 \mathrm{kU}_{\mathrm{A}} / \mathrm{L}$ ) to peanut components and peanut extract per DBPCFC result (i.e. positive with objective symptoms, positive with subjective symptoms and negative) and the titers of sensitization. Patients with a positive DBPCFC result with objective symptoms recognized Ara $h$ 1, 2, 3 and 6 more often and had a higher titer compared to patients with subjective symptoms or a negative DBPCFC result. A large proportion of patients recognized Ara h 8 (75\%) and peanut extract (89\%), irrespective of the DBPCFC result.

\section{Positive objective}

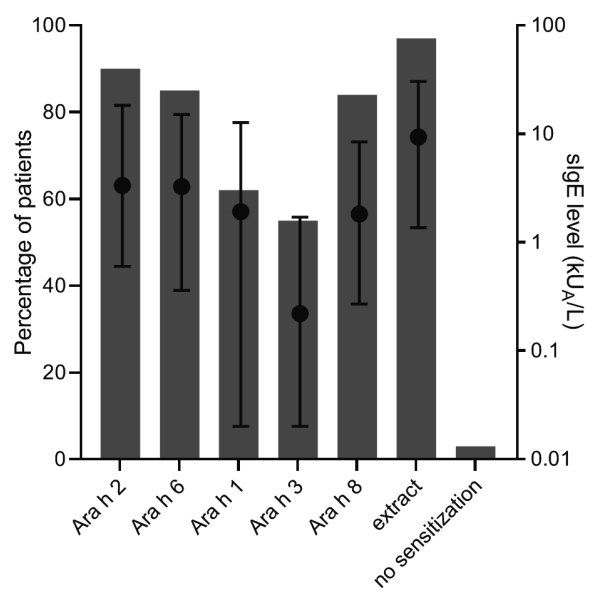

Negative

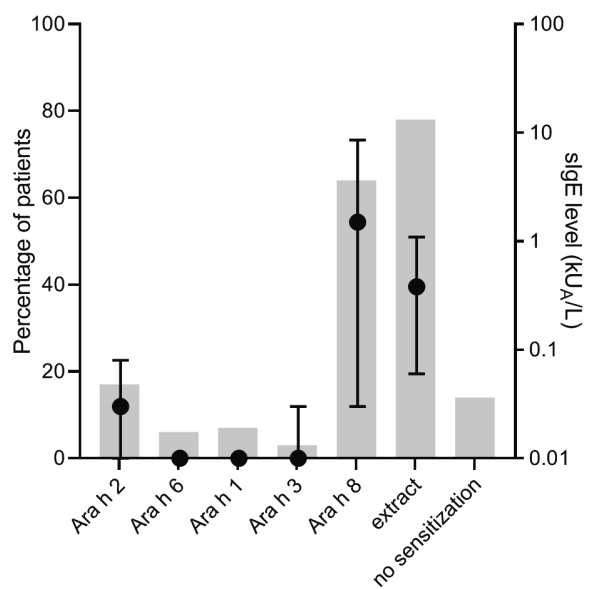

\section{Positive subjective}

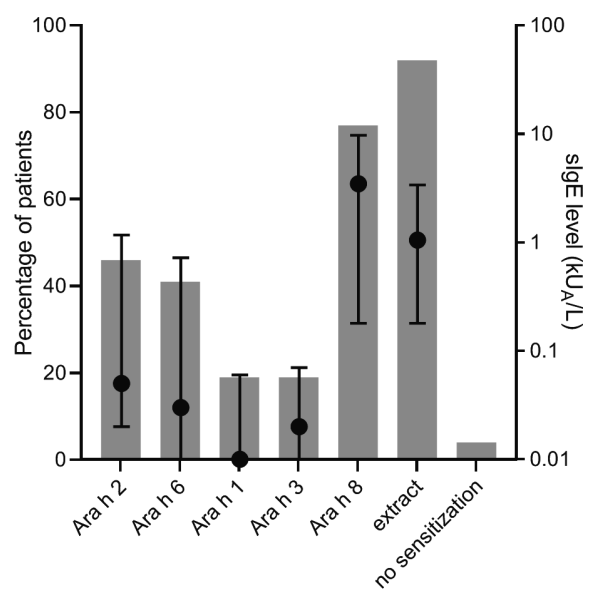

Figure 2. Percentage of patients with sensitization to peanut extract or peanut components and titers of sensitization, per DBPCFC result

Sensitization is defined as a value of $\geq 0.10 \mathrm{kU}_{\mathrm{A}} / \mathrm{L}$. Median (interquartile range) titers of $\operatorname{sigE}$ are displayed on logarithmic scales (base 10).

Abbreviations: DBPCFC, double-blind placebo-controlled food challenge; slgE, specific lgE. 


\section{Peanut-allergic patients without sensitization to Ara h 1, 2, 3 and 6}

In $16(17 \%)$ patients with a positive DBPCFC result we did not detect sensitization to Ara h 1, 2, 3 and 6 (Figure 3). Five of 16 patients had objective symptoms Mueller grade 3 during the DBPCFC. Peanut-allergic patients with undetectable sensitization to Ara h $1,2,3$ and 6 were sensitized to Ara $\mathrm{h} 8$ and peanut extract $(n=8)$, to Ara h 8 only $(n=2)$, to peanut extract only $(n=2)$ or were not sensitized to peanut extract or any of the peanut components $(n=4)$.

Additional diagnostic testing on a line blot (EUROLINE) was performed, measuring Ara $\mathrm{h} 1,2,3,5,6,7,8,9,10,11,14$ and 15, in 14 of 16 patients with leftover serum available after the ImmunoCAP measurements. Again, no sensitization to Ara $\mathrm{h} 1,2$, 3 or 6 was measured on the line blot. Furthermore, all patients were tested negative to Ara h 5, 7, 10, 11, 14 and 15. Two patients were sensitized to Ara h 9 (patient ID 3 and ID 14 in Figure 3) on the line blot.

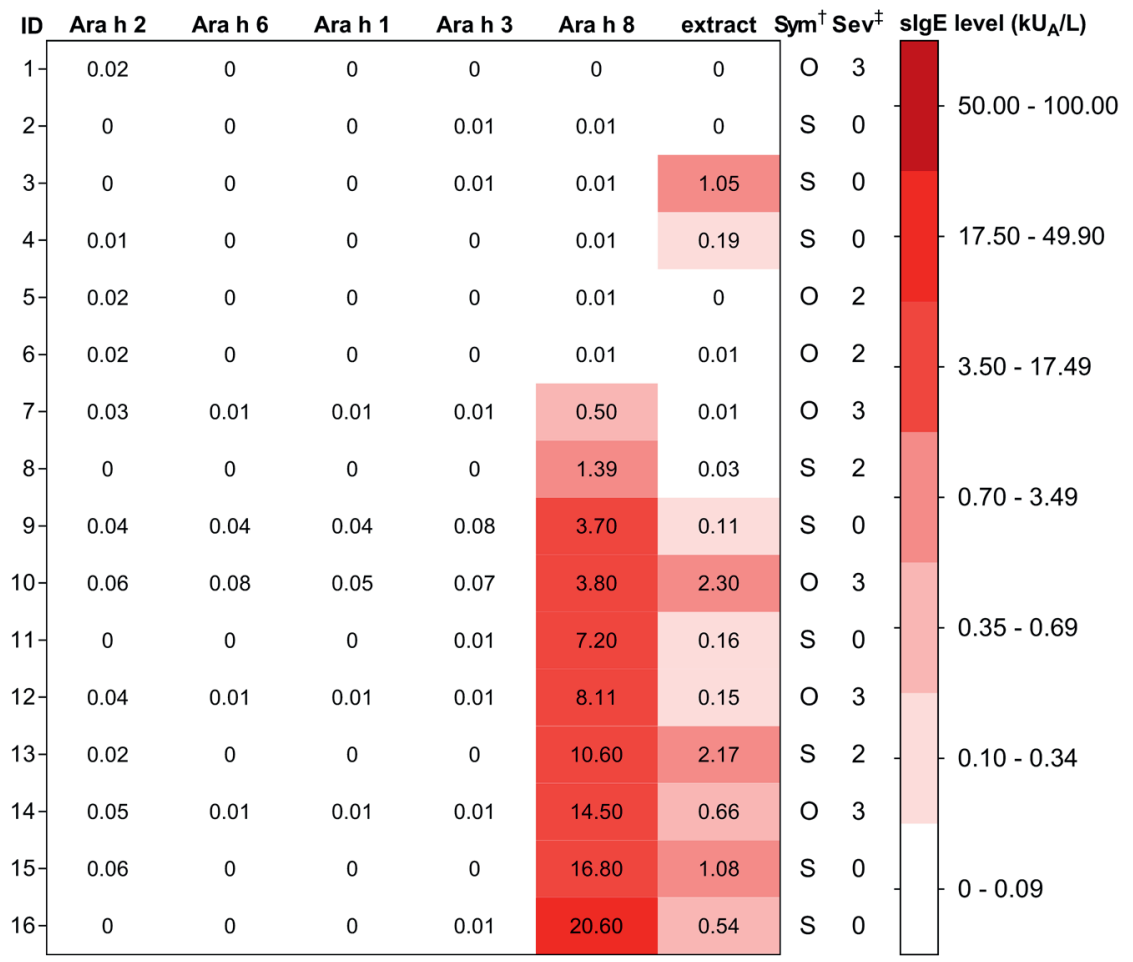

Figure 3. Sensitization pattern in 16 peanut-allergic patients without sensitization to Ara h 2 and Ara h 6 † Symptoms during the oral food challenge: S, subjective; O, objective.

* Severity of symptoms during the oral food challenge based on the Mueller score with 0. Oral symptoms, 2. Gastro-intestinal symptoms and 3. Respiratory symptoms. ${ }^{16,30}$

Abbreviations: ID, patient number; O, objective; S, subjective; Sev, severity; Sym, symptoms. 


\section{Discussion}

In the current study, we showed that Ara h 2 and Ara h 6 are the best predictors of peanut allergy in adults with suspected peanut allergy. Our validated Ara $\mathrm{h} 2$ cutoff level with $100 \%$ positive predictive value (PPV) (cutoff $\geq 1.75 \mathrm{kU}_{\mathrm{A}} / \mathrm{L}$ ) can be used to accurately predict peanut allergy in one third of patients. Furthermore, we were the first to investigate the diagnostic value of sIgE to Ara $\mathrm{h} 6$ using a singleplex assay in adults and were able to identify a cutoff level of Ara h 6 with 100\% PPV (cutoff $\geq$ $\left.1.80 \mathrm{kU}_{\mathrm{A}} / \mathrm{L}\right)$, again applicable to almost one third of patients.

Our results are in line with previous research that identified Ara h 2 and Ara h 6 as the best predictors of peanut allergy. ${ }^{8,14,15}$ The AUC value of sIgE to Ara $h 2$ was lower in our study in adults (AUC 0.85) compared to the AUC values reported in studies in children (AUC 0.90-0.96). ${ }^{14,34}$ In addition, we observed a lower cutoff level of Ara $\mathrm{h} 2$ with $100 \%$ PPV (cutoff $\geq 1.75 \mathrm{kU}_{\mathrm{A}} / \mathrm{L}$ ) compared to children (cutoff 4.0-5.0 $\mathrm{kU}_{\mathrm{A}} / \mathrm{L}$ ). ${ }^{8,35}$ The Ara $\mathrm{h} 2$ cutoff with $100 \%$ PPV was even lower in the subset of patients who were not part of the previous diagnostic study and underwent a DBPCFC between 2012 and 2019 (cutoff $\geq 0.43 \mathrm{kU}_{\mathrm{A}} / \mathrm{L}$ ). The AUC value of sIgE to Ara h 6 using a singleplex assay in our study (AUC 0.85) was comparable to the AUC value of SIgE to Ara h 6 using a multiplex assay in adults (AUC 0.82), but lower than observed in children using a multiplex assay (AUC 0.98). ${ }^{14,15}$ The lower diagnostic value of peanut components in adults compared to children could be explained by the higher proportion of birch pollen-related peanut allergy in adults. In patients with birch pollen allergy, cross-sensitization of Ara $\mathrm{h} 8$ with major birch pollen allergen Bet $v 1$ occurs and these patients have mainly mild or subjective symptoms after peanut ingestion, making it more difficult to discriminate between presence and absence of allergy. ${ }^{12,14,16}$ As shown in the current study, adult peanut-allergic patients with subjective symptoms were less often sensitized to Ara h 2 and Ara h 6 and had lower titers, resulting in a lower diagnostic value. Further insight into the levels of IgE sensitization over time, from childhood to adulthood, could contribute to a better understanding of the differences between children and adults.

The concentrations of sIgE to Ara h 2 and Ara h 6 were highly correlated in our and previous studies. ${ }^{36,37}$ The extensive overlap in sIgE reactivity and comparable diagnostic accuracy, indicate that ImmunoCAP results for either Ara h 2 or Ara h 6 are sufficient in the diagnostic work-up of a suspected peanut allergy in adults. Multivariable analyses revealed that a combination of Ara $\mathrm{h} 1$ and Ara $\mathrm{h} 6$ had the highest diagnostic accuracy, with an AUC comparable to those of Ara h 2 and Ara $h$ 6 alone, despite regularization (i.e. shrinkage of the coefficients) (AUC 0.85). At present, however, we recommend using sIgE reactivity to peanut component Ara $\mathrm{h}$ 2 in daily practice, as the cutoff level is validated in the current study, 
implementation in daily practice is straightforward, and interpretation of sIgE-levels to one peanut component is more intuitive than of a clinical prediction rule based on the Lasso model.

Thirty-two percent of patients could be classified correctly as peanut-allergic when using the validated cutoff level of sIgE to Ara $\mathrm{h} 2$ with $100 \%$ positive predictive value (PPV) (cutoff $\geq 1.75 \mathrm{kU}_{\mathrm{A}} / \mathrm{L}$ ). This cutoff level of Ara $\mathrm{h} 2$ could be used in daily clinical practice to select patients for oral food challenges. In patients with an Ara $h$ 2 level above the cutoff level, peanut allergy is diagnosed with (almost) 100\% accuracy without an oral food challenge. In these patients, a 1-day oral food challenge may still be performed, in accordance with the patient and physician preference, to assess the severity of the allergic reaction and the eliciting dose. In patients with an Ara $\mathrm{h} 2$ level below the cutoff level, peanut-allergic status cannot be predicted. In these patients, a 2-day double-blind placebo-controlled food challenge is needed to confirm or exclude peanut allergy.

It must be noted that sIgE to Ara $\mathrm{h} 2$ and Ara $\mathrm{h} 6$ cannot be used to rule out peanut allergy in adults. In 16 peanut-allergic patients (17\%) in our cohort we did not detect sensitization to Ara h 2 and Ara h 6, nor for Ara h 1 or Ara h 3. In these patients, sensitization to peanut allergens might not have been detected by the ImmunoCAP platform or peanut allergy might have been caused by sensitization to other peanut components. Therefore, a different diagnostic testing modality was used to test a large number of peanut components (Ara h 1, 2, 3, 5, 6, 7, 8, 9, 10, 11, 14 and 15), resulting in 2 of 16 patients recognizing Ara h 9. Ten of 16 (63\%) patients were sensitized to the birch pollen related allergen Ara $h$, and 3 of these 10 patients that only recognized Ara $\mathrm{h} 8$ experienced objective respiratory symptoms during the double-blind placebo-controlled food challenge (DBPCFC). However, the sensitization rate to Ara $\mathrm{h} 8$ in peanut-tolerant patients was comparable (64\%), thus sIgE to Ara $\mathrm{h} 8$ was not predictive of clinical reactivity to peanut. A comparable frequency (18\%) of allergic patients with negative serology tests, including Ara h 1, 2, 3 and 6, was reported in peanut-allergic patients in the EuroPrevall study. ${ }^{36}$ The authors hypothesized that peanut oleosins may have caused allergic reactions in these patients. Interestingly, all peanut-allergic patients with negative Ara h 1, 2, 3 and 6 results in our study were tested negative to peanut oleosins (i.e. Ara h 10, 11, 14 and 15). Thus, peanut allergy in non-sensitized patients as detected in this and previous studies might be caused by sensitization to thus far unidentified peanut components.

A limitation of our study was that we only included patients from a tertiary care center with a conclusive DBPCFC result, and with leftover serum available. However, we believe our sample was a representative sample as we did not observe significant differences between included and excluded patients. Furthermore, the 
percentage of positive DBPCFCs in our study (62\%) was comparable to other studies (43-66\%). ${ }^{16,38,39}$ Strengths of our study were that we included a large cohort of adult patients who all underwent a standardized DBPCFC for peanut allergy, we measured a large number of peanut components, we performed a validation of our previous results and were able to confirm these findings. ${ }^{16}$

In conclusion, we showed that specific IgE to Ara h 2 and Ara h 6 are the best predictors of peanut allergy in adults. Furthermore, we validated our previously published Ara h 2 cutoff level with 100\% positive predictive value. The validated Ara h 2 cutoff level should be implemented in daily practice to reduce the need for double-blind placebo-controlled food challenges, as all adults with an Ara $\mathrm{h} 2 \geq$ $1.75 \mathrm{kU}_{\mathrm{A}} / \mathrm{L}$ were peanut-allergic.

\section{Acknowledgments}

We thank Lisette Bok and Angelique Kooij for performing ImmunoCAP measurements. We thank Mark Smits for performing EUROLINE measurements and Paco Welsing for giving statistical advice. We acknowledge Rob Klemans for providing data from the previous diagnostic study. ImmunoCAP material was provided by Thermo Fisher Scientific (Uppsala, Sweden). The EUROLINE material was provided by EUROIMMUN (Lübeck, Germany). 


\section{References}

1. Nwaru BI, Hickstein L, Panesar SS, Roberts G, Muraro A, Sheikh A. Prevalence of common food allergies in Europe: A systematic review and meta-analysis. Allergy. 2014;69(8):992-1007.

2. Lyons SA, Burney PGJ, Ballmer-Weber BK, et al. Food Allergy in Adults: Substantial Variation in Prevalence and Causative Foods Across Europe. J Allergy Clin Immunol Pract. 2019;7(6):19201928.

3. Soller L, Ben-Shoshan M, Harrington DW, et al. Adjusting for nonresponse bias corrects overestimates of food allergy prevalence. J Allergy Clin Immunol Pract. 2015;3(2):291-293.e2.

4. Muraro A, Werfel T, Hoffmann-Sommergruber K, et al. EAACI Food Allergy and Anaphylaxis Guidelines: Diagnosis and management of food allergy. Allergy. 2014;69(8):1008-1025.

5. Niggeman B, Yürek S, Beyer K. Severe anaphylaxis requiring intensive care during oral food challenge-It is not always peanuts. Pediatr Allergy Immunol. 2017;28(2):201-203.

6. Koplin JJ, Perrett KP, Sampson HA. Diagnosing Peanut Allergy with Fewer Oral Food Challenges. J Allergy Clin Immunol Pract. 2019;7(2):375-380.

7. Dang TD, Tang $M$, Choo $S$, et al. Increasing the accuracy of peanut allergy diagnosis by using Ara h 2. J Allergy Clin Immunol. 2012;129(4):1056-1063.

8. van Erp FC, Knol EF, Pontoppidan B, Meijer Y, van der Ent CK, Knulst AC. The IgE and basophil responses to Ara h 2 and Ara h 6 are good predictors of peanut allergy in children. J Allergy Clin Immunol. 2017;139(1):358-360.e8.

9. van Erp FC, Klemans RJB, Meijer $Y$, et al. Using Component-Resolved Diagnostics in the Management of Peanut-Allergic Patients. Curr Treat Options Allergy. 2016:169-180.

10. Hazebrouck S, Guillon B, Paty E, Dreskin SC, Adel-Patient K, Bernard H. Variable IgE crossreactivity between peanut $2 \mathrm{~S}$-albumins: The case for measuring IgE to both Ara $\mathrm{h} 2$ and Ara $\mathrm{h} 6$. Clin Exp Allergy. 2019;49(8):1107-1115.

11. Blankestijn MA, Otten HG, Suer W, Weimann A, Knol EF, Knulst AC. Specific IgE to peanut $2 \mathrm{~S}$ albumin Ara h 7 has a discriminative ability comparable to Ara h 2 and 6. Clin Exp Allergy. 2017;:1-6.

12. Beyer $\mathrm{K}$, Grabenhenrich $\mathrm{L}$, Härtl $\mathrm{M}$, et al. Predictive values of component-specific IgE for the outcome of peanut and hazelnut food challenges in children. Allergy Eur J Allergy Clin Immunol. 2015;70(1):90-98.

13. Ebisawa $M$, Moverare $R$, Sato $S$, Borres MP, Ito $K$. The predictive relationship between peanutand Ara h 2-specific serum IgE concentrations and peanut allergy. J Allergy Clin Immunol Pr. 2015;3(1):131-2.e1.

14. Kukkonen AK, Pelkonen AS, Makinen-Kiljunen S, Voutilainen $\mathrm{H}$, Makela MJ. Ara h 2 and Ara 6 are the best predictors of severe peanut allergy: a double-blind placebo-controlled study. Allergy. 2015;70(10):1239-1245.

15. Klemans RJB, Knol EF, Bruijnzeel-Koomen CAFM, Knulst AC. The diagnostic accuracy of specific IgE to Ara $\mathrm{h} 6$ in adults is as good as Ara h 2. Allergy. 2014;69(8):1112-1114.

16. Klemans RJB, Broekman $\mathrm{HCHP}$, Knol EF, et al. Ara $\mathrm{h} 2$ is the best predictor for peanut allergy in adults. J Allergy Clin Immunol Pract. 2013;1(6):632-638.e1.

17. Chapmah $M$, Pomés A, Breiteneder $H$, Ferreira F. Nomenclature and structural biology of allergens. J Allergy Clin Immunol. 2007;119:414-420.

18. Koppelman SJ, de Jong GAH, Laaper-Ertmann $\mathrm{M}$, et al. Purification and immunoglobulin Ebinding properties of peanut allergen Ara h 6: evidence for cross-reactivity with Ara h 2. Clin Exp Allergy. 2005;35(4):490-497.

19. Burks AW, Cockrell G, Stanley JS, Helm RM, Bannon GA. Recombinant peanut allergen Ara h I expression and IgE binding in patients with peanut hypersensitivity. I Clin Invest. 1995;96(4):1715-1721.

20. Rabjohn P, Helm EM, Stanley JS, et al. Molecular cloning and epitope analysis of the peanut allergen Ara h 3. J Clin Invest. 1999;103(4):535-542. 
21. Mittag D, Akkerdaas J, Ballmer-Weber BK, et al. Ara h 8, a Bet v 1-homologous allergen from peanut, is a major allergen in patients with combined birch pollen and peanut allergy. J Allergy Clin Immunol. 2004;114(6):1410-1417.

22. Asarnoj A, Nilsson C, Lidholm J, et al. Peanut component Ara h 8 sensitization and tolerance to peanut. J Allergy Clin Immunol. 2012;130(2):468-472.

23. Lauer I, Dueringer $\mathrm{N}$, Pokoj $\mathrm{S}$, et al. The non-specific lipid transfer protein, Ara $\mathrm{h} 9$, is an important allergen in peanut. Clin Exp Allergy. 2009;39(9):1427-1437.

24. Agabriel C, Ghazouani O, Birnbaum J, et al. Ara h 2 and Ara h 6 sensitization predicts peanut allergy in Mediterranean pediatric patients. Pediatr Allergy Immunol. 2014;25(7):662-667.

25. Asarnoj A, Glaumann S, Elfstrom L, et al. Anaphylaxis to peanut in a patient predominantly sensitized to Ara h 6. Int Arch Allergy Immunol. 2012;159(2):209-212.

26. Canonica GW, Ansotegui IJ, Pawankar R, et al. A WAO-ARIA-GA2LEN consensus document on molecular-based allergy diagnostics. World Allergy Organ J. 2013;6(1):1.

27. Peeters KABM, Koppelman SJ, Van Hoffen E, et al. Does skin prick test reactivity to purified allergens correlate with clinical severity of peanut allergy? Clin Exp Allergy. 2007;37(1):108-115.

28. Taylor SL, Hefle SL, Bindslev-Jensen C, et al. A consensus protocol for the determination of the threshold doses for allergenic foods: how much is too much? Clin Exp Allergy. 2004;34(5):689695.

29. Vlieg-Boerstra BJ, Herpertz I, Pasker L, et al. Validation of novel recipes for double-blind, placebo-controlled food challenges in children and adults. Allergy Eur J Allergy Clin Immunol. 2011;66(7):948-954.

30. Mueller HL. Diagnosis and Treatment of Insect Sensitivity. J Asthma Res. 1966;3(4):331-333.

31. DeLong ER., DeLong DM., Clarke-Pearson DL. Comparing the Areas under Two or More Correlated Receiver Operating Characteristic Curves: A Nonparametric Approach. Biometrics. 1988;44(3):837-845.

32. Tibshirani R. Regression Shrinkage and Selection via the Lasso. J Roy Stat Soc B. 1996;58(1):267288.

33. Dhand NK, Khatkar MS. Statulator: An online statistical calculator. Sample Size Calculator for Estimating a Single Proportion. 2014.

34. Klemans RJB, Otte $D, K n o l ~ M$, et al. The diagnostic value of specific IgE to Ara $\mathrm{h} 2$ to predict peanut allergy in children is comparable to a validated and updated diagnostic prediction model. J Allergy Clin Immunol. 2013;131(1):157-163.

35. Kim HY, Han Y, Kim K, et al. Diagnostic value of specific IgE to peanut and ara $\mathrm{h} 2$ in Korean children with peanut allergy. Allergy, Asthma Immunol Res. 2016;8(2):156-160.

36. Ballmer-Weber BK, Lidholm J, Fernandez-Rivas $M$, et al. IgE recognition patterns in peanut allergy are age dependent: perspectives of the EuroPrevall study. Allergy. 2015;70(4):391-407.

37. Koid AE, Chapman MD, Hamilton RG, et al. Ara h 6 complements Ara h 2 as an important marker for IgE reactivity to peanut. J Agric Food Chem. 2014;62(1):206-213.

38. Zambrano Ibarra G, Fuentes Aparicio V, Infante Herrero S, Blanca M, Zapatero Remon L. Peanut Allergy in Spanish Children: Comparative Profile of Peanut Allergy versus Tolerance. Int Arch Allergy Immunol. 2019;178(4):370-376.

39. Grabenhenrich $L$, Lange $L$, Härtl $M$, et al. The component-specific to total IgE ratios do not improve peanut and hazelnut allergy diagnoses. J Allergy Clin Immunol. 2016;137(6):17511760.e8. 


\section{Supplemental tables}

Supplemental Table 1. Baseline characteristics of included and excluded patients

\begin{tabular}{|c|c|c|c|c|}
\hline & Included & Excluded & $\mathrm{n}$ available & $P$-value \\
\hline n (\%) & $154(75)$ & $50(25)$ & & \\
\hline Age, mean (SD) & $27(22-38)$ & $24.5(20.3-31)$ & 32 & 0.055 \\
\hline Male gender & $52(34)$ & $19(38)$ & 50 & 0.585 \\
\hline Allergic rhinitis $^{+}$ & $52(75)$ & $11(73)$ & 15 & 0.869 \\
\hline \multicolumn{5}{|l|}{ Sensitization, median (IQR) } \\
\hline $\operatorname{sIgE}$ peanut & $1.65(0.35-11.05)$ & $2.35(0.71-22.40)$ & 23 & 0.377 \\
\hline sIgE Ara h 1 & $0.02(0.00-0.61)$ & $0.00(0.00-0.01)$ & 4 & - \\
\hline sIgE Ara h 2 & $0.16(0.03-3.50)$ & $0.38(0.00-1.37)$ & 19 & 0.424 \\
\hline sIgE Ara h 3 & $0.03(0.01-0.18)$ & $0.00(0.00-0.01)$ & 4 & - \\
\hline sIgE Ara h 6 & $0.07(0.00-3.14)$ & $0.02(0.00-0.53)$ & 6 & - \\
\hline sIgE Ara h 8 & $2.02(0.11-8.57)$ & $1.23(0.01-8.75)$ & 18 & 0.428 \\
\hline DBPCFC result ${ }^{\ddagger}$ & & & 50 & $<0.0005$ \\
\hline Positive & $95(62)$ & $20(40)$ & & \\
\hline Inconclusive & 0 & $18(36)$ & & \\
\hline Negative & $59(38)$ & $12(24)$ & & 0.213 \\
\hline Severity positive DBPCFC & & & 11 & \\
\hline Mueller 0 & $15(16)$ & $2(18)$ & & \\
\hline Mueller 1 & $16(17)$ & $2(18)$ & & \\
\hline Mueller 2 & $32(34)$ & $7(64)$ & & \\
\hline Mueller 3 & $28(30)$ & 0 & & \\
\hline Mueller 4 & $2(2)$ & 0 & & \\
\hline
\end{tabular}

Values are numbers (\%), unless otherwise indicated.

${ }^{+}$Missing data included patients $\mathrm{n}=85$

${ }^{\ddagger}$ One of the reasons for exclusion was an inconclusive DBCPFC result. The proportion of positive and negative DBPCFC results were comparable between groups $(p=0.931)$.

${ }^{\S}$ Missing data included patients $n=2$. Severity of symptoms during the oral food challenge based on the Mueller score with 0 . oral symptoms, 1 . cutaneous symptoms, 2. gastrointestinal symptoms, 3 . respiratory symptoms and 4 . cardiovascular symptoms.

Abbreviations: $D B P C F C$, double-blind placebo-controlled food challenge; $I Q R$, interquartile range; $S D$, standard deviation; slgE, specific IgE.

Supplemental Table 2. Diagnostic value of peanut components in the subset of the cohort with available sIgE to Ara h $6(n=118$ of 154)

\begin{tabular}{ll}
\hline & AUC (95\% CI) \\
\hline sIgE extract & $0.76(0.68-0.85)$ \\
sIgE Ara h 1 & $0.76(0.68-0.84)$ \\
sIgE Ara h 2 & $0.85(0.78-0.92)$ \\
sIgE Ara h 3 & $0.75(0.67-0.84)$ \\
sIgE Ara h 8 & $0.57(0.46-0.68)$ \\
\hline
\end{tabular}

Abbreviations: $A \cup C$, area under the curve; $\mathrm{Cl}$, confidence interval; slgE, specific IgE. 
Chapter 3

Supplemental Table 3. DeLong's test for comparing two correlated ROC curves

\begin{tabular}{lccccc}
\hline & SIgE & sIgE & sIgE & sIgE & sIgE \\
& Ara h 6 & extract & Ara h 1 & Ara h 3 & Ara h 8 \\
\hline sIgE Ara h 2 & 0.87 & $\mathbf{0 . 0 2}$ & $<\mathbf{0 . 0 1}$ & $<\mathbf{0 . 0 1}$ & $<\mathbf{0 . 0 1}$ \\
sIgE Ara h 6 & & $\mathbf{0 . 0 2}$ & $<\mathbf{0 . 0 1}$ & $<\mathbf{0 . 0 1}$ & $<\mathbf{0 . 0 1}$ \\
SIgE extract & & & 0.61 & 0.56 & $<\mathbf{0 . 0 1}$ \\
SIgE Ara h 1 & & & & 0.93 & $<\mathbf{0 . 0 1}$ \\
sIgE Ara h 3 & & & & & $<\mathbf{0 . 0 1}$ \\
sIgE Ara h 8 & & & & & \\
\hline
\end{tabular}

Data are $P$-values corrected for multiple testing using the Benjamini-Hochberg procedure. Significant $P$-values are in boldface.

Abbreviations: slgE, specific lgE. 
Peanut components in adults 
$r$

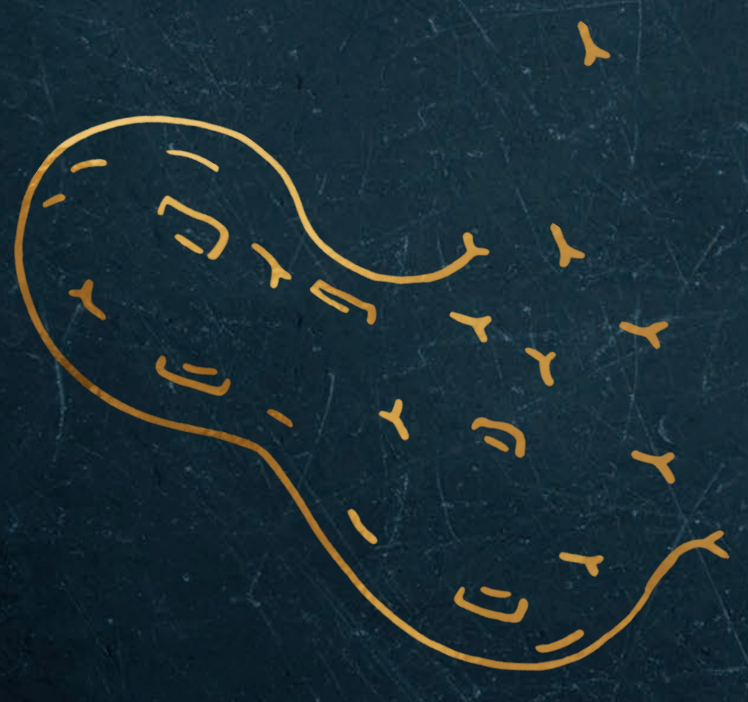

$\downarrow$

$\alpha$ 


\section{Chapter 4}

\section{Three-year follow-up after peanut food challenges: accidental reactions in allergic children and introduction failure in tolerant children}




\section{Abstract}

\section{Background}

Long-term follow-up data after peanut double-blind placebo-controlled food challenges (DBPCFC) are limited. We sought to evaluate 1) accidental allergic reactions to peanut and health-related quality of life (HRQL) in peanut-allergic children and 2) introduction of peanut and HRQL in peanut-tolerant children 3 years after a DBPCFC.

\section{Methods}

We conducted a 3-year follow-up of 83 children who participated in a prospective study in the Netherlands and all underwent a DBPCFC for peanut. Three years after the DBPCFC, all parents and children aged 8 years or older were asked to fill out a questionnaire on accidental allergic reactions, current diet and the Food Allergy Quality of Life Questionnaire-Parent Form.

\section{Results}

In total, parents of 69 (83\%) children with a mean (SD) age of 11.5 (3.5) years completed the questionnaire $3.1(0.4)$ years after a peanut DBPCFC. Seventeen (41\%) of 41 peanut-allergic children had a total of 68 allergic reactions during 3year follow-up. None of 7 out of 17 children with severe lower respiratory symptoms used their epinephrine auto-injector. Nine (32\%) of 28 peanut-tolerant children failed to introduce peanut. HRQL only improved significantly and clinically relevant in peanut-tolerant children after successful peanut introduction.

\section{Conclusion}

Despite adequate diagnostic procedures and guidance, living with peanut allergy remains a challenge during childhood. In peanut-tolerant children peanut introduction often fails. However, peanut introduction seems meaningful to improve HRQL. 


\section{Introduction}

Peanut allergy is one of the most common food allergies among children. ${ }^{1}$ The gold standard to diagnose peanut allergy is a double-blind placebo-controlled food challenge (DBPCFC). ${ }^{2}$ Children and parents are advised to avoid peanut after a positive DBPCFC result or to introduce peanut after a negative DBPCFC result. Limited data exist on the long-term follow-up after a DBPCFC, including the frequency and treatment of accidental allergic reactions, diet and health-related quality of life (HRQL).

Children with confirmed peanut allergy have frequent accidental allergic reactions and often do not recognize and adequately treat allergic symptoms. ${ }^{3-6}$ For children with peanut allergy in Canada, the annual incidence rate of accidental reactions to peanut ranged from 10 to $15 \% .{ }^{4,7}$ However, most children with peanut allergy participating in these studies were diagnosed based on a convincing clinical history and/or sensitization. It is unclear how often children have an allergic reaction after the explicit advice to avoid peanut following a positive DBPCFC result and how these allergic reactions are treated. On the other hand, children with a negative DBPCFC result are advised to consume peanuts regularly to maintain oral tolerance. Children who continue to avoid peanut after outgrowing a peanut allergy may be at risk for recurrence of food allergy. ${ }^{8,9}$ Previous studies showed that children and parents often fail to introduce peanut at home. ${ }^{10-13}$ We found that $38 \%$ of children failed to introduce peanut at home one month after a negative DBPCFC result despite guided introduction including a 7-day introduction schedule, telephonic counseling and a diary. ${ }^{14}$ Children may fail to introduce peanut into the diet due to aversion to the food that long has been eliminated, a false-negative oral food challenge result or fear of persistent allergy. ${ }^{10,14}$ It is unclear how often children fail to introduce peanut years after a negative DBPCFC result including standardized guided introduction.

Children and parents might benefit from a DBPCFC because our previous systematic review reported that HRQL improved up to 6 months after an oral food challenge, irrespective of the outcome. ${ }^{15} \mathrm{HRQL}$ up to years after an oral food challenge and $\mathrm{HRQL}$ after peanut introduction have not been previously reported.

Limited data exist on the long-term follow-up after a DBPCFC, including the frequency and treatment of accidental allergic reactions, diet and HRQL. We sought to evaluate 1) the frequency and nature of accidental allergic reactions and HRQL in peanut-allergic children; 2) peanut introduction and HRQL in peanut-tolerant children. 


\section{Methods}

\section{Participants and procedures}

The current study is a 3-year follow-up of 83 children who participated in a prospective diagnostic study performed in one tertiary care and one secondary care center in the Netherlands between January 2012 and May 2015. The previous study included children aged 3 to 18 years with a suspected peanut allergy based on a previous reaction and/or sensitization. ${ }^{16}$ All children underwent a DBPCFC for peanut. A DBPCFC was considered positive when objective or severe long-lasting subjective symptoms occurred. Children with a positive DBPCFC result $(n=45 ; 54 \%)$ were instructed to eliminate peanut and were educated when and how to use emergency treatment. Children with a negative DBPCFC result followed by a negative or inconclusive open challenge with 10 whole peanuts $(n=38 ; 46 \%)$ were instructed to introduce peanut at home according to a standardized, carefully monitored guided introduction during one month. ${ }^{14}$ Successful short-term introduction into the diet $(n=23 ; 62 \%)$ was defined as parental-reported ingestion of at least $5 \mathrm{~g}$ of whole peanuts or an equivalent at home during the introduction period and eating products containing peanuts as an ingredient on a regular basis ( $\geq 2$ times a month). Among children with failed short-term introduction $(n=14$; $37 \%), 3$ children were considered peanut-allergic because of repetitive and persistent oral itch within 5 minutes after all peanut ingestions. In conclusion, 48 children (58\%) were considered peanut-allergic and 35 children (42\%) were considered peanut-tolerant (Figure 1).

Three years after the DBPCFC for peanut, all parents and children aged 8 years or older were contacted by mail and were asked to fill out an electronic questionnaire on accidental allergic reactions, current diet and HRQL. Families who did not respond were reminded within 2 and/or 4 weeks of the mailing and contacted by telephone. The study was reviewed and approved by the ethical committee of the University Medical Centre Utrecht and written informed consent was obtained from parents and children before enrollment in the original study. 


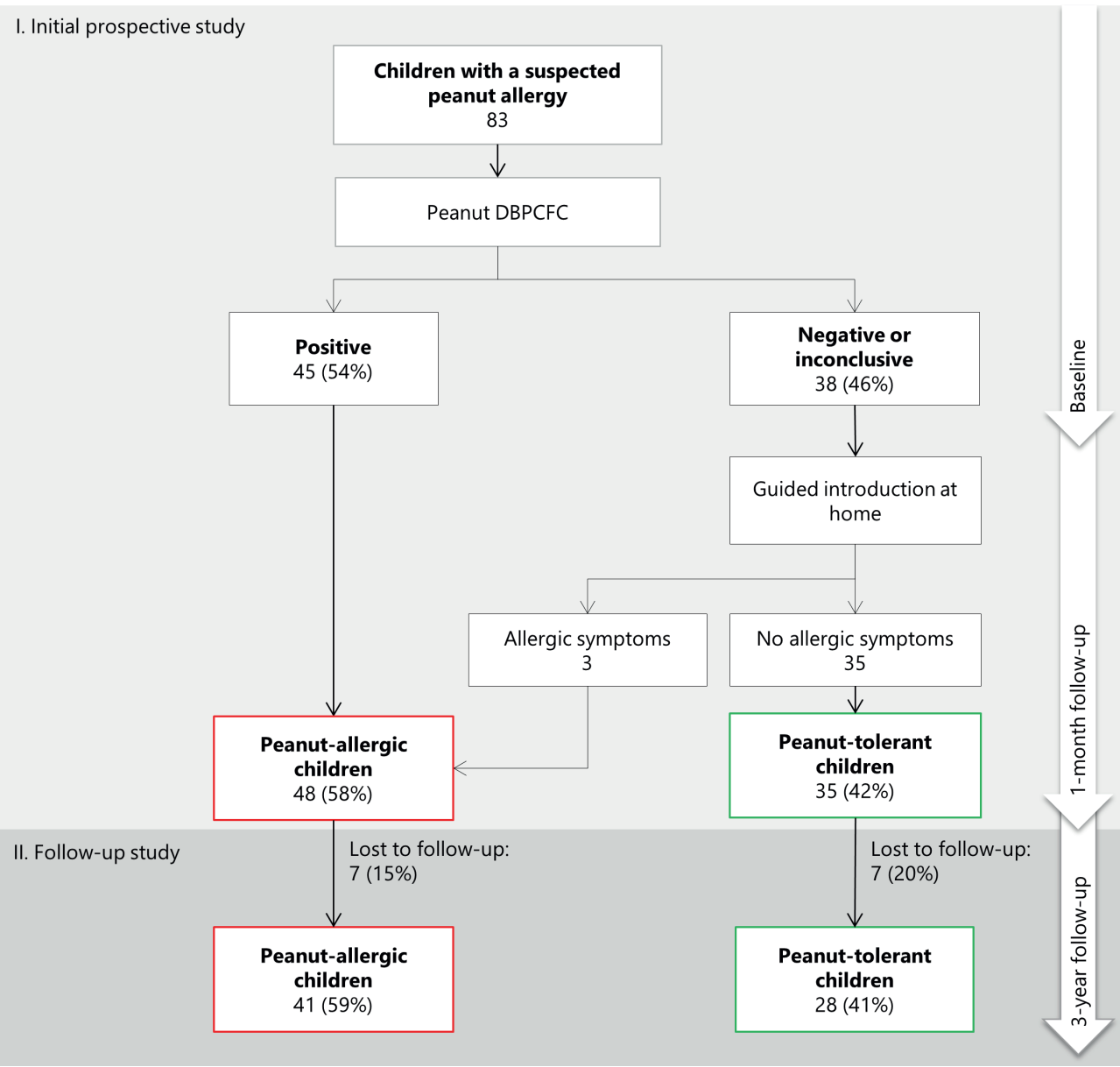

Figure 1. Study flowchart

Values are numbers (\%).

Abbreviations: DBPCFC, double-blind placebo-controlled food challenge.

\section{Accidental allergic reactions in peanut-allergic children}

All parents were asked whether their child has had any accidental allergic reaction to peanut since the DBPCFC for peanut. If any allergic reaction to peanut was reported, parents were asked to report on the most severe allergic reaction. Details collected on the most severe allergic reaction to peanut included the food ingested, estimated amount consumed, time interval from ingestion of the product to reaction, symptoms, duration, potential cofactors and treatment. The symptoms were categorized according to the PRACTALL consensus report. ${ }^{17}$ The severity of symptoms was categorized according to the Sampson classification of anaphylaxis as grade 1 to $5 .^{18}$ Grade 1 to 3 was considered mild or moderate and grade 4 to 5 severe. 


\section{Introduction of peanut in peanut-tolerant children}

Details collected on the current diet included the question whether the child consumed whole peanuts or products with visible peanut (category a), products with peanut as an ingredient (category $b$ ) or products with precautionary allergen labelling e.g. 'may contain' or 'manufactured on a shared line with products containing peanut' (category $c$ ). If children avoided one of the peanut product categories, details on the reasons for avoidance were collected. Successful longterm introduction of peanut was defined as eating category a and/or category $b$ products at least once a month.

\section{Health-related quality of life}

Health-related quality of life (HRQL) was assessed using the Food Allergy Quality of Life Questionnaires (FAQLQ) before and 3 years after the DBPCFC for peanut. The FAQLQ is a self-reported, disease-specific instrument to evaluate the impact of food allergy on patients' HRQL. For this study, the FAQLQ parent form (FAQLQ-PF) for parents of all children, the child form (FAQLQ-CF) for children aged 8 to 13 years and the teenager form (FAQLQ-TF) for children aged 13 to 18 years were used. ${ }^{19-21}$ The FAQLQ-PF contains 30 items and 3 domains (emotional impact, foodrelated anxiety, social dietary limitations), the FAQLQ-CF contains 24 items and 4 domains (allergen avoidance, risk of accidental exposure, emotional impact, dietary restrictions) and the FAQLQ-TF contains 23 items and 3 domains (allergen avoidance, risk of accidental exposure, emotional impact). All items are scored on a seven-point scale ranging from 1 to 7 , with 1 as the best possible score. The relevance of the change in FAQLQ-scores was evaluated using the minimal clinically important difference (MCID) of $0.50 .^{22}$

\section{Statistical analysis}

Data were presented as mean (standard deviation [SD]) or median (interquartile range [IQR]) for continuous data, and numbers (percentages) for categorical variables. Differences between responders and non-responders, between peanutallergic and peanut-tolerant children and between children with and without allergic reactions during follow-up were statistically evaluated by the chi-square test for categorical variables and the independent samples t-test or Mann Whitney $\mathrm{U}$ test for continuous variables with Bonferroni correction for multiple testing. The annual incidence rate of accidental allergic reactions was expressed as the number of allergic reactions divided by the sum of the patient years (time from DBPCFC to follow-up). The difference between FAQLQ scores at baseline and follow-up were compared using the paired t-test. A $P$-value $<0.05$ was considered statistically significant. All analyses were performed using SPSS for Windows (version 25). 


\section{Results}

\section{Patient characteristics}

In total, 69 (83\%) of 83 children completed the questionnaire mean (SD) 3.1 (SD, $0.4)$ years after a positive $(n=41 / 48)$ or negative $(n=28 / 35)$ DBPCFC result (Figure 1$)$. The mean (SD) age of the children at follow-up was 11.5 (3.5) years and $48(70 \%)$ were male (Table 1). Children who completed the questionnaire and children who did not were comparable in age, gender, sensitization, HRQL, DBPCFC results and DBPCFC severity (Supplemental Table 1).

Table 1. Baseline characteristics

\begin{tabular}{|c|c|c|c|c|}
\hline & All children & $\begin{array}{l}\text { Peanut-allergic } \\
\text { children }\end{array}$ & $\begin{array}{l}\text { Peanut-tolerant } \\
\text { children }\end{array}$ & $P$-value \\
\hline $\mathrm{n}$ & 69 & $41(59)$ & $28(41)$ & \\
\hline \multicolumn{5}{|l|}{ Baseline characteristics } \\
\hline Age at DBPCFC, mean (SD) & $8.4(3.5)$ & $8.6(3.4)$ & $8.2(3.7)$ & 0.624 \\
\hline Age at follow-up, mean (SD) & $11.5(3.5)$ & $11.7(3.3)$ & $11.2(3.7)$ & 0.614 \\
\hline Male gender & $48(70)$ & $27(66)$ & $21(75)$ & 0.417 \\
\hline \multicolumn{5}{|l|}{ History of atopy } \\
\hline Asthma & $33(48)$ & $23(56)$ & $10(36)$ & 0.096 \\
\hline Allergic rhinitis & $41(59)$ & $27(66)$ & $14(50)$ & 0.188 \\
\hline Atopic dermatitis & $40(58)$ & $23(56)$ & $17(61)$ & 0.703 \\
\hline $\begin{array}{l}\text { Avoidance other foods than } \\
\text { peanut }\end{array}$ & & & & 0.169 \\
\hline 0 foods & $25(36)$ & $13(32)$ & $12(43)$ & \\
\hline 1 or 2 foods & $10(15)$ & $6(15)$ & $4(14)$ & \\
\hline 3 or 4 foods & $11(16)$ & $4(10)$ & $7(25)$ & \\
\hline 5 or 6 foods & $15(22)$ & $12(29)$ & $3(11)$ & \\
\hline$\geq 7$ foods & $8(12)$ & $6(15)$ & $2(7)$ & \\
\hline
\end{tabular}

Values are numbers (\%), unless otherwise indicated.

Abbreviations: $D B P C F C$, double-blind placebo-controlled food challenge; $n$, number; SD, standard deviation.

\section{Accidental allergic reactions in peanut-allergic children}

Seventeen (41\%) of 41 children with peanut allergy had 68 accidental allergic reactions during the 3 -year follow-up. Three children with peanut allergy (aged < 12 years) experienced 5 or more allergic reactions during the follow-up. Children with peanut allergy and allergic reactions to peanut during follow-up were comparable to with those without accidental allergic reactions in age, gender, history of asthma, duration of elimination diet for peanut, sensitization, elimination diet for other allergens, severity of symptoms during the DBPCFC, eliciting dose of the DBPCFC and dietary advice following the DBPCFC (Supplemental Table 3). The frequency of accidental allergic reactions did not differ between children 12 years or younger and children 13 years or older. Parents of children with any allergic reaction during follow-up were asked to report the characteristics of the most 
severe reaction (Table 2 and 3). All children developed symptoms within 2 hours from ingestion. Most children consumed unpacked foods (8 of 17 children) or products with peanut as an ingredient (6 of 17 children). Five of 17 children had mild or moderate symptoms and used antihistamines at home. Twelve of 17 children reported severe symptoms and used antihistamines $(n=7)$, antihistamines and oral steroids $(n=1)$, a bronchodilator $(n=1)$ or used no medication at all $(n=3)$. More than half of (parents of) children with severe symptoms did not consult a medical professional. Only two children with severe symptoms consulted a general practitioner and one child was admitted to the hospital. Seven of 17 children reported severe lower respiratory symptoms. None of these 7 children used their epinephrine auto-injector.

Table 2. Characteristics of most severe accidental allergic reactions to peanut in 17 (41\%) of 41 peanutallergic children

\begin{tabular}{|c|c|c|}
\hline & & n (\%) \\
\hline \multirow[t]{4}{*}{ Number of reactions } & 1 or 2 & $9(53)$ \\
\hline & 3 or 4 & $4(25)$ \\
\hline & $\geq 5$ & $3(18)$ \\
\hline & Missing data & $1(6)$ \\
\hline \multirow[t]{2}{*}{ Age category } & Children ( $\leq 12$ years) & $11(65)$ \\
\hline & Adolescents (>12 years) & $6(35)$ \\
\hline \multirow[t]{3}{*}{ Contact } & Ingestion & $14(82)$ \\
\hline & In oral cavity & $1(6)$ \\
\hline & Skin contact & $2(12)$ \\
\hline \multirow[t]{2}{*}{ Time from ingestion to symptoms } & $\leq 2$ hours & $17(100)$ \\
\hline & $>2$ hours & 0 \\
\hline \multirow[t]{5}{*}{ Symptoms according to PRACTALL ${ }^{16}$} & Skin & $10(59)$ \\
\hline & Gastro-intestinal & $16(94)$ \\
\hline & Upper respiratory & $8(47)$ \\
\hline & Lower respiratory & $7(41)$ \\
\hline & Cardiovascular/neurologic & $9(53)$ \\
\hline \multirow[t]{6}{*}{ Severity of symptoms ${ }^{+}$} & Mild or moderate & \\
\hline & Grade 1 & $1(6)$ \\
\hline & Grade 2 & $3(18)$ \\
\hline & Grade 3 & $1(6)$ \\
\hline & Severe & \\
\hline & Grade 4 & $12(71)$ \\
\hline \multirow[t]{5}{*}{ Treatment } & No medicines & $3(18)$ \\
\hline & Antihistamines & $12(71)$ \\
\hline & Antihistamines + oral steroids & $1(6)$ \\
\hline & Epinephrine auto-injector & 0 \\
\hline & Bronchodilators & $1(6)$ \\
\hline \multirow[t]{3}{*}{ Consultation with physician } & No consultation & $14(82)$ \\
\hline & GP visit & $2(12)$ \\
\hline & Hospital/ED visit & $1(6)$ \\
\hline
\end{tabular}

${ }^{\dagger}$ According to Sampson classification of anaphylaxis. ${ }^{18}$

Abbrevations: $n$, number. 
Table 3. Characteristics of most severe allergic reaction during follow-up per patient

\begin{tabular}{|c|c|c|c|c|c|}
\hline ID & Product & Label & All symptoms reported $^{+}$ & $\mathbf{S}^{\ddagger}$ & Treatment \\
\hline $1^{\S}$ & Baklava & UNP & $\begin{array}{l}\text { Pruritis, oral itch, feel of oral swelling, nausea, } \\
\text { diarrhea, abdominal pain, dyspnea, feel of } \\
\text { collapse }\end{array}$ & 4 & $\mathrm{AH}$ \\
\hline $2^{\S}$ & $\begin{array}{l}\text { Peanut skin } \\
\text { contact }\end{array}$ & UNP & $\begin{array}{l}\text { Pruritis, swelling eyes, diarrhea, blocked } \\
\text { nose, wheezing }\end{array}$ & 4 & INH \\
\hline 3 & $\begin{array}{l}\text { Japanese } \\
\text { rice crackers }\end{array}$ & ING & $\begin{array}{l}\text { Pruritis, rash, oral itch, nausea, vomiting, } \\
\text { diarrhea, abdominal pain, changed activity }\end{array}$ & 4 & None \\
\hline $4^{\S}$ & Peanut & UNP & $\begin{array}{l}\text { Rash, urticaria, swelling eyes, feel of oral } \\
\text { swelling, nausea, vomiting, abdominal pain, } \\
\text { blocked nose, dyspnea, anxiety }\end{array}$ & 4 & $\mathrm{AH}$ \\
\hline 5 & Chocolate & UNP & $\begin{array}{l}\text { Pruritis, rash, urticaria, diarrhea, blocked } \\
\text { nose }\end{array}$ & 4 & None \\
\hline 6 & Fries & UNP & $\begin{array}{l}\text { Swelling eyes, oral itch, nausea, diarrhea, } \\
\text { abdominal pain }\end{array}$ & 4 & $\mathrm{AH}$ \\
\hline 7 & Cake & UNP & Swelling eyes, vomiting, abdominal pain & 2 & $\mathrm{AH}$ \\
\hline $8^{\S}$ & Biscuit & ING & $\begin{array}{l}\text { Swelling eyes, feel of oral swelling, nausea, } \\
\text { abdominal pain, dysphagia, anxiety }\end{array}$ & 4 & $\mathrm{AH}+\mathrm{OS}$ \\
\hline 9 & Biscuit & ING & Oral itch, nausea, abdominal pain & 2 & $\mathrm{AH}$ \\
\hline $10^{\S}$ & $\begin{array}{l}\text { Pesto with } \\
\text { cashew nut }\end{array}$ & PAL & $\begin{array}{l}\text { Oral itch, feel of oral swelling, nausea, } \\
\text { abdominal pain, sneezing, cough, anxiety }\end{array}$ & 4 & $\mathrm{AH}$ \\
\hline 11 & $\begin{array}{l}\text { Peanut } \\
\text { butter }\end{array}$ & UNP & Oral itch, abdominal pain & 1 & $\mathrm{AH}$ \\
\hline 12 & UNK & UNP & Nausea, diarrhea, abdominal pain & 4 & None \\
\hline 13 & Potato chips & CON & Pruritis, nausea, dizziness, feel of collapse & 4 & $\mathrm{AH}$ \\
\hline 14 & $\begin{array}{l}\text { Peanut } \\
\text { butter }\end{array}$ & ING & Pruritis, rash, swelling eyes, itching eye & 2 & $\mathrm{AH}$ \\
\hline $15^{\S}$ & Biscuit & ING & $\begin{array}{l}\text { Oral itch, nausea, abdominal pain, itching eyes, } \\
\text { feel of oral swelling, hoarseness, cough, } \\
\text { dysphagia, dyspnea, dizziness }\end{array}$ & 4 & $\mathrm{AH}$ \\
\hline $16^{\S}$ & Salty sticks & ING & $\begin{array}{l}\text { Pruritis, rash, urticaria, swelling eyes, itching } \\
\text { eyes, nausea, abdominal pain, blocked nose, } \\
\text { sneezing, dyspnea, changed activity }\end{array}$ & 4 & $\mathrm{AH}$ \\
\hline 17 & Biscuit & PAL & $\begin{array}{l}\text { Pruritis, rash, urticaria, swelling eyes, oral } \\
\text { itch, itching eyes, blocked nose, sneezing, } \\
\text { anxiety }\end{array}$ & 3 & $\mathrm{AH}$ \\
\hline \multicolumn{6}{|c|}{$\begin{array}{l}\text { Patient-reported symptoms. Objective symptoms are in boldface. } \\
\text { Severity of symptoms according to Sampson classification of anaphylaxis. }{ }^{18} \\
\text { Severe lower respiratory symptoms requiring the use of epinephrine. } \\
\text { mentioned as an ingredient; INH, bronchodilator; OS, oral steroids; PAL, precautionary allergen labelling } \\
\text { for peanut; S, severity according to Sampson classification of anaphylaxis; UNP, unpacked foods; UNK, } \\
\text { unknown. }\end{array}$} \\
\hline
\end{tabular}




\section{Unchanged quality of life in peanut-allergic children after follow-up}

Parent-proxy-reported HRQL remained unchanged between baseline and 3-year follow-up in children with peanut allergy (mean difference [MD], 0.05; $P=0.823$ ). There was no significant difference in HRQL between children with or without allergic reactions during follow-up, or between children $\leq 12$ years or $>12$ years of age. On the individual level, HRQL improved in $29 \%$ and deteriorated in $39 \%$ of children with peanut allergy 3 years after the DBPCFC compared with before. HRQL data are presented in Figure 2 and Supplemental Table 4.

\section{Introduction of peanut in peanut-tolerant children}

Long-term introduction of peanut 3 years after the DBPCFC failed in 9 (32\%) of 28 peanut-tolerant children (Figure 4). Five (28\%) children with successful short-term guided introduction failed to introduce peanut on the long-term. The reported reasons for failure were aversion $(n=4)$ or aversion and fear $(n=1)$. Three children (33\%) with failed short-term guided introduction also failed to introduce peanut on the long-term due to aversion $(n=1)$, aversion and fear $(n=1)$ or fear $(n=1)$. One child did not start guided introduction and failed to introduce peanut on the longterm due to fear. The consumption of the different peanut product categories among children with successful and failed introduction is displayed in Figure 3. Only one child (4\%) did not consume products with precautionary allergen labeling (category c) 3 years after a negative DBPCFC.

Parent-proxy-reported HRQL improved significantly between baseline and 3-year follow-up in peanut-tolerant children (MD, $-0.51 ; P=0.025)$ (Supplemental Table 4). This change was considered clinically relevant as the minimal import difference of 0.50 was exceeded. A stratified analysis per long-term introduction outcome in peanut-tolerant children showed that HRQL improved significantly and clinically relevant after successful long-term introduction (MD -0.70; $p=0.013)$ and not after failed long-term introduction (MD, $-0.05 ; P=0.906$ ). In addition, domain HRQL scores improved clinically relevant after successful long-term introduction (Supplemental Table 4). On the individual level, HRQL improved in 52\% and deteriorated in $19 \%$ of peanut-tolerant children 3 years after the DBPCFC compared with before (Figure 2). 


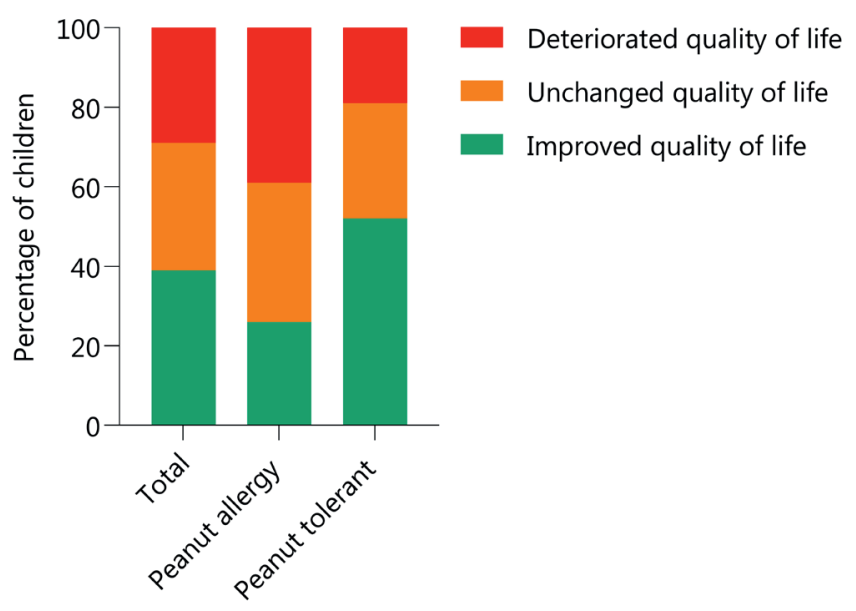

Figure 2. Percentage of children with an improved (change $<-0.5$ ), unchanged (change -0.5 to 0.5 ) or deteriorated (change $>0.5$ ) HRQL 3 years after a DBPCFC for peanut

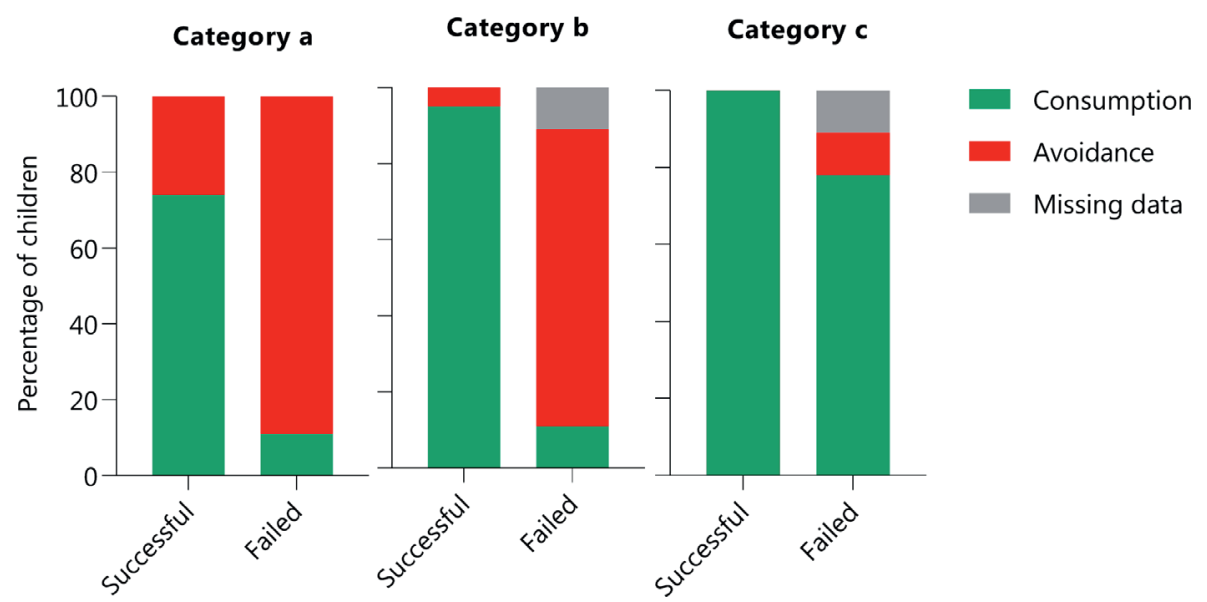

Figure 3. Consumption of peanut in peanut-tolerant children with successful $(n=19)$ or failed $(n=9)$ long-term introduction

Products category $a$, whole or visible peanut; Category b, peanut as an ingredient; Category c, precautionary allergen labelling. 


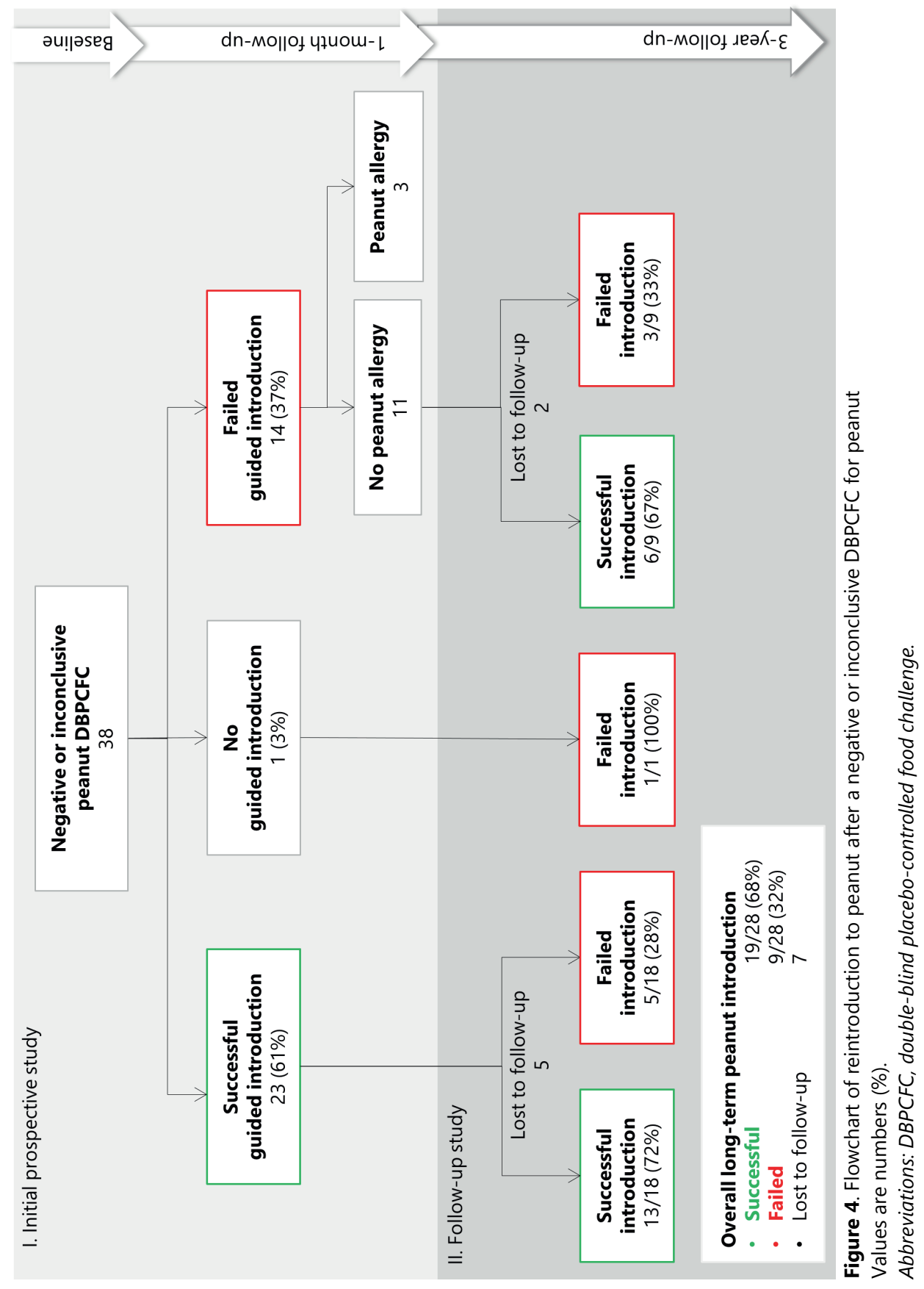




\section{Discussion}

To our knowledge, this is the first study investigating the long-term follow-up after a DBPCFC for peanut in children. In this study we have shown that almost half of peanut-allergic children have accidental reactions during 3 years after a positive DBPCFC result and that severe accidental reactions are not treated with epinephrine. In addition, peanut-tolerant children often experience failure of peanut introduction into the diet 3 years after a negative DBPCFC result. Our results indicate that $\mathrm{HRQL}$ improves in peanut-tolerant children after successful peanut introduction and not after failed peanut introduction or in peanut-allergic children.

The high rate of accidental allergic reactions despite the explicit advice to avoid peanut, as seen in this study, is of clinical concern. Previous studies reported that up to $60 \%$ of children with peanut allergy (diagnosed using clinical history and/or sensitization) had accidental allergic reactions to peanut over a median period of 5 to 6 years. ${ }^{23,24}$ Children may have frequent accidental allergic reactions because foods are often unpacked, labels are misread by children or parents, products are inadequately labelled or because of risky behavior of the child. ${ }^{25,26}$ Another point of concern is that none of the children with a severe accidental allergic reaction requiring epinephrine used an epinephrine auto-injector upon peanut exposure although all children were prescribed an auto-injector and were instructed on how and when to use the device. Children and parents may not have used an epinephrine auto-injector because they were not carrying the device or failed to recognize allergic reactions and anaphylaxis. A recent analysis of the European Anaphylaxis Register demonstrated that more than $85 \%$ of patients with severe anaphylaxis did not use their epinephrine auto-injectors despite clear recommendations. ${ }^{27}$

Previous studies demonstrated a beneficial impact of a positive oral food challenge result on parent-proxy-reported HRQL up to 6 months after the challenge. ${ }^{22,28}$ In the current long-term follow-up study, we found no difference between HRQL at baseline and 3 years after a positive DBPCFC result for peanut. Although we did not measure HRQL between these two time points, our results suggest that the beneficial impact of a positive DBPCFC result on HRQL may wane over time. Psychologists may have a role to support children and their families as psychological or psychosocial interventions have been shown to improve HRQL and reduce feelings of anxiety. ${ }^{29,30}$ Furthermore, children with peanut allergy and their families may benefit from multidisciplinary allergy care including regular clinical consultations with an experienced physician and dietitian to evaluate any accidental ingestion of peanut, continue education on peanut avoidance and the use of emergency medications and adapt counselling to the child's age. ${ }^{31,32}$ 
Our study showed that almost one third of peanut-tolerant children failed to introduce peanut into the diet 3 years after the DBPCFC, despite guidance after the DBPCFC. Guidance after a negative DBPCFC result may not influence introduction of the allergen as suggested by our data and by a recent retrospective study in 128 children after a negative DBPCFC result for cow's milk. ${ }^{33}$ The rate of introduction of cow's milk did not differ between children with or without follow-up by phone or in person. Children failed to introduce peanut into the diet mainly because of aversion to peanut, although alternative products with hidden peanut were offered in the first month after the DBPCFC. Children may dislike peanuts as the taste of peanut has previously been paired with illness, they are not used to the taste and texture of peanuts or because they have long been eliminating peanut. The importance of peanut introduction in peanut-tolerant children is supported by our results that indicate that parent-proxy-reported HRQL improved only in peanut-tolerant children after successful peanut introduction and remained unchanged after failed peanut introduction. Further research and discussion with experts and patient organizations is needed to investigate whether food aversion and anxiety, which were the main reason not to introduce, may be relieved when psychological guidance is provided.

Our study is limited by a relatively small sample size limiting the possibility to evaluate predictors of accidental allergic reactions, introduction failure and HRQL. In addition, due to the small sample size we were not able to analyze childreported HRQL per allergic status and per introduction success or failure group. It has been suggested that parents report better HRQL than children themselves. ${ }^{34}$ Thus, our results on HRQL are limited to the parent perspective and further research is needed to assess the long-term impact of a DBPCFC and peanut introduction on the child's HRQL. Furthermore, we may have underestimated or overestimated the rate and severity of accidental allergic reactions due to recall bias over a 3-year time period. The study is strengthened by a prospective study design, including a well-defined patient cohort of children who all underwent a DBPCFC for peanut and a high response rate after 3-year follow-up.

In conclusion, despite adequate diagnostic procedures and guidance, living with peanut allergy remains a challenge during childhood. In peanut-tolerant children peanut introduction often fails. However, peanut introduction is meaningful to improve health-related quality of life.

\section{Acknowledgments}

We thank Coco Dekkers for performing the data analysis on HRQL. 


\section{References}

1. Nwaru BI, Hickstein L, Panesar SS, et al. The epidemiology of food allergy in Europe: A systematic review and meta-analysis. Allergy. 2014;69(1):62-75.

2. Muraro A, Werfel T, Hoffmann-Sommergruber K, et al. EAACI Food Allergy and Anaphylaxis Guidelines: Diagnosis and management of food allergy. Allergy. 2014;69(8):1008-1025.

3. Versluis A, Knulst AC, Kruizinga AG, et al. Frequency, severity and causes of unexpected allergic reactions to food: A systematic literature review. Clin Exp Allergy. 2015;45(2):347-367.

4. $\quad \mathrm{Yu} J \mathrm{~W}, \mathrm{Kagan} \mathrm{R}$, Verreault $\mathrm{N}$, et al. Accidental ingestions in children with peanut allergy. $J$ Allergy Clin Immunol. 2006;118(2):466-472.

5. Mehl A, Wahn U, Niggemann B. Anaphylactic reactions in children--a questionnaire-based survey in Germany. Allergy. 2005;60(11):1440-1445.

6. Muraro A, Roberts G, Clark A, et al. The management of anaphylaxis in childhood: position paper of the European academy of allergology and clinical immunology. Allergy. 2007;62(8):857-871.

7. Nguyen-Luu NU, Ben-Shoshan M, Alizadehfar R, et al. Inadvertent exposures in children with peanut allergy. Pediatr Allergy Immunol. 2012;23(2):133-139.

8. Busse PJ, Nowak-Wegrzyn AH, Noone SA, Sampson HA, Sicherer SH. Recurrent peanut allergy. N Engl J Med. 2002;347(19):1535-1536.

9. Fleischer DM, Conover-Walker MK, Christie L, Burks AW, Wood RA. Peanut allergy: recurrence and its management. J Allergy Clin Immunol. 2004;114(5):1195-1201.

10. Eigenmann PA, Caubet JC, Zamora SA. Continuing food-avoidance diets after negative food challenges. Pediatr Allergy Immunol. 2006:601-605.

11. Gau J, Wang J. Rate of food introduction after a negative oral food challenge in the pediatric population. J Allergy Clin Immunol Pract. 2017;5(2):475-476.

12. van Erp FC, Boot J, Knulst AC, Pasmans SGM, van der Ent CK, Meijer Y. Reintroduction failure after negative peanut challenges in children. Pediatr Allergy Immunol. 2014;25(6):580-585.

13. van der Valk JPM, van Wijk RG, Vergouwe $Y$, de Jong NW. Failure of introduction of food allergens after negative oral food challenge tests in children. Eur J Pediatr. 2015;174(8):10931099.

14. van Erp FC, Knulst AC, Gorissen DMW, Kok IL, van der Ent CK, Meijer Y. Guided introduction after negative double-blind placebo-controlled peanut challenges in children. J Allergy Clin Immunol Pract. 2017;5(2):489-493.e1.

15. Kansen HM, Le TM, Meijer Y, Flokstra-de Blok BMJ, Welsing PMJ, van der Ent CK, et al. The impact of oral food challenges for food allergy on quality of life: a systematic review. Pediatr Allergy Immunol. 2018;29(5):527-537

16. van Erp FC, Knol EF, Pontoppidan B, Meijer Y, van der Ent CK, Knulst AC. The IgE and basophil responses to Ara h 2 and Ara h 6 are good predictors of peanut allergy in children. $J$ Allergy Clin Immunol. 2017;139(1):358-360.e8.

17. Sampson HA, Wijk G Van, Bindslev-jensen C, Sicherer S. Standardizing double-blind, placebocontrolled oral food challenges: American Academy of Allergy, Asthma \& Immunology European Academy of Allergy and Clinical Immunology PRACTALL consensus report. J Allergy Clin Immunol. 2012;130(6):1260-1274.

18. Sampson HA. Anaphylaxis and emergency treatment. Pediatrics. 2003;111:1601-1608.

19. Flokstra-De Blok BMJ, DunnGalvin a., Vlieg-Boerstra BJ, et al. Development and validation of a self-administered Food Allergy Quality of Life Questionnaire for children. Clin Exp Allergy. 2009;39(1):127-137.

20. Flokstra-de Blok BMJ, DunnGalvin A, Vlieg-Boerstra BJ, et al. Development and validation of the self-administered Food Allergy Quality of Life Questionnaire for adolescents. J Allergy Clin Immunol. 2008;122(1).

21. DunnGalvin A, De BlokFlokstra BMJ, Burks AW, Dubois AEJ, Hourihane JOB. Food allergy QoL 
questionnaire for children aged 0-12 years: Content, construct, and cross-cultural validity. Clin Exp Allergy. 2008;38(6):977-986.

22. Dunngalvin A, Cullinane C, Daly DA, Blok BMJF, Dubois AEJ, Hourihane JOB. Longitudinal validity and responsiveness of the Food Allergy Quality of Life Questionnaire - Parent Form in children $0-12$ years following positive and negative food challenges. Clin Exp Allergy. 2010:476-485.

23. Leek TK Vander, Liu AH, Stefanski K, Blacker B, Bock SA. The natural history of peanut allergy in young children and its association with serum peanut-specific IgE. J Pediatr. 2000;137(6):749755.

24. Sicherer SH, Burks AW, Sampson HA. Clinical features of acute allergic reactions to peanut and tree nuts in children. Pediatrics. 1998;102(1):e6.

25. Blom WM, Michelsen-Huisman $A D$, van Os-Medendorp $H$, et al. Accidental food allergy reactions: Products and undeclared ingredients. J Allergy Clin Immunol. 2018;142(3):865-875.

26. Warren $\mathrm{CM}$, Dyer AA, Otto AK, et al. Food Allergy-Related Risk-Taking and Management Behaviors Among Adolescents and Young Adults. J Allergy Clin Immunol Pract. 2017;5(2):381390.e13.

27. Grabenhenrich LB, Dölle $S$, Ruëff $F$, et al. Epinephrine in Severe Allergic Reactions: The European Anaphylaxis Register. J Allergy Clin Immunol Pract. 2018;6(6):1898-1906.e1.

28. Soller $\mathrm{L}$, Hourihane J, Dunngalvin A. The impact of oral food challenge tests on food allergy health-related quality of life. Allergy. 2014;69(9):1255-1257.

29. Knibb RC, Semper H. Impact of suspected food allergy on emotional distress and family life of parents prior to allergy diagnosis. Pediatr Allergy Immunol. 2013;24(8):798-803.

30. Knibb RC, Hourihane JOB. The psychosocial impact of an activity holiday for young children with severe food allergy: A longitudinal study. Pediatr Allergy Immunol. 2013;24(4):368-375.

31. Venter $\mathrm{C}$, Sicherer SH, Greenhawt M. Management of Peanut Allergy. J Allergy Clin Immunol Pract. 2019;7(2):345-355.e2.

32. Skypala I, De Jong NW, Angier E, et al. Promoting and achieving excellence in the delivery of Integrated Allergy Care: The European Academy of Allergy \& Clinical Immunology competencies for allied health professionals working in allergy. Clin Transl Allergy. 2018;8(1):611.

33. Schrijvers $M$, Hendriks $T$, Mars $H$, Bertrams-Maartens IA. The effect of follow-up after a negative double-blinded placebo-controlled cow's milk challenge on successful reintroduction. Eur $J$ Pediatr. 2019;178(7):1113-1117.

34. Van der Velde JL, Flokstra-de Blok BMJ, Dunngalvin a., Hourihane JOB, Duiverman EJ, Dubois a. EJ. Parents report better health-related quality of life for their food-allergic children than children themselves. Clin Exp Allergy. 2011;41(10):1431-1439. 


\section{Supplemental tables}

Supplemental Table 1. Comparison of children who completed the questionnaire and those who did not

\begin{tabular}{llll}
\hline & $\begin{array}{l}\text { 3-year } \\
\text { follow-up }\end{array}$ & $\begin{array}{l}\text { No 3-year } \\
\text { follow-up }\end{array}$ & P-value $^{\dagger}$ \\
\hline $\mathrm{n}$ (\%) & $69(83)$ & $14(17)$ & \\
Age at follow-up, mean (SD) & $11.5(3.5)$ & $12.1(3.6)$ & 0.547 \\
Male gender & $48(70)$ & $11(79)$ & 0.748 \\
sIgE to peanut, median (IQR) & $4.00(0.78-13.05)$ & $2.15(0.19-17.35)$ & 0.458 \\
sIgE to Ara h 2, median (IQR) & $1.02(0.17-8.95)$ & $0.85(0.05-8.63)$ & 0.401 \\
DBPCFC characteristics & & & \\
FAQLQ-PF score before DBPCFC, mean (SD) & $2.40(0.95)$ & $2.80(1.53)$ & 0.319 \\
Results DBPCFC & & & \\
$\quad$ Positive & $41(59)$ & $7(50)$ & 0.563 \\
$\quad 28(41)$ & $7(50)$ & \\
Pegative & & & \\
Positive DBPCFC & & 0.236 \\
Reaction during DBPCFC & & \\
$\quad$ Grade 1 & $5(12)$ & $2(29)$ & \\
$\quad$ Grade 2 & $19(46)$ & $1(14)$ & \\
$\quad$ Grade 3 & $9(22)$ & $3(43)$ & \\
$\quad$ Grade 4 & $6(15)$ & $1(14)$ & \\
Missing & $2(5)$ & & \\
Negative DBPCFC & & $5(71)$ & \\
Introduction of peanut after one month & $18(64)$ & $2(29)$ & \\
$\quad$ Successful & $9(32)$ & 0 & \\
Failed & $1(4)$ &
\end{tabular}

Values are numbers (\%), unless otherwise indicated.

${ }^{+} P$-values of less than 0.006 taken to be significant according to Bonferroni correction for multiple testing.

${ }^{\ddagger}$ According to Sampson classification of anaphylaxis. ${ }^{17}$

Abbreviations: DBPCFC, double-blind placebo-controlled food challenge; FAQLQ-PF, Food Allergy Quality of Life Questionnaire - Parent Form; IQR, interquartile range; $n$, number; $S D$, standard deviation. 
Supplemental Table 2. Comparison between peanut-allergic children with and without accidental reactions during follow-up

\begin{tabular}{|c|c|c|c|}
\hline & $\begin{array}{l}\text { Accidental } \\
\text { reaction(s) } \\
\mathrm{n}(\%)\end{array}$ & $\begin{array}{l}\text { No accidental } \\
\text { reaction } \\
\mathrm{n}(\%)\end{array}$ & $\boldsymbol{P}_{\text {-value }}{ }^{+}$ \\
\hline $\mathrm{n}(\%)$ & $17(41)$ & $24(59)$ & \\
\hline \multicolumn{4}{|l|}{ Baseline characteristics } \\
\hline Age at follow-up & & & 1.000 \\
\hline 4 to 13 years & $11(65)$ & $16(67)$ & \\
\hline 13 to 18 years & $6(35)$ & $8(33)$ & \\
\hline Male gender & $11(65)$ & $16(67)$ & 1.000 \\
\hline \multicolumn{4}{|l|}{ History of atopy } \\
\hline Duration of peanut elimination diet & & & 0.972 \\
\hline 3 to 8 years & $6(35)$ & $9(38)$ & \\
\hline 8 to 13 years & $7(41)$ & $9(38)$ & \\
\hline Lifelong & $4(24)$ & $6(25)$ & \\
\hline Previous reaction to peanut ${ }^{\ddagger}$ & & & 0.615 \\
\hline Never ingested peanut & $3(18)$ & $7(29)$ & \\
\hline Contact & $3(18)$ & $2(8)$ & \\
\hline Grade 1-3 & $5(29)$ & $9(38)$ & \\
\hline Grade 4-5 & $6(35)$ & $6(25)$ & \\
\hline Elimination diet $>1$ other allergen & $12(71)$ & $16(67)$ & 1.000 \\
\hline History of asthma & $8(47)$ & $15(63)$ & 0.358 \\
\hline \multicolumn{4}{|l|}{ Sensitization before DBPCFC } \\
\hline sIgE to peanut, median (IQR) & 8.3 (2.7 to 21.45$)$ & 7.1 (1.54 to 43.50$)$ & 0.853 \\
\hline sIgE to Ara h 2, median (IQR) & $6.4(2.36$ to 23.85$)$ & $3.45(0.93$ to 32.50$)$ & 0.662 \\
\hline \multicolumn{4}{|l|}{ DBPCFC } \\
\hline FAQLQ-PF score before DBPCFC, mean (SD) & $2.37(1.00)$ & $2.35(1.01)$ & 0.950 \\
\hline Reaction during $\mathrm{DBPCFC}^{\ddagger}$ & & & 0.565 \\
\hline Grade 1-3 & $12(71)$ & $20(83)$ & \\
\hline Grade 4-5 & $3(18)$ & $3(13)$ & \\
\hline Missing & $2(12)$ & $1(4)$ & \\
\hline \multicolumn{4}{|l|}{ LOAEL DBPCFC, median (IQR) } \\
\hline Subjective & 65 (1 to 1950$)$ & $100(20$ to 1000$)$ & 0.609 \\
\hline Objective & $1000(0$ to 2500$)$ & 1000 (38 to 1000$)$ & 0.939 \\
\hline Dietary advice: no products with PAL & $7(41)$ & $6(25)$ & 0.322 \\
\hline
\end{tabular}

${ }^{\dagger} P$-values of less than 0.003 taken to be significant according to Bonferroni correction for multiple testing.

${ }^{*}$ According to Sampson classification of anaphylaxis. ${ }^{17}$

Abbreviations: DBPCFC, double-blind placebo-controlled food challenge; FAQLQ-PF, Food Allergy Quality of Life Questionnaire - Parent Form; IQR, interquartile range; LOAEL, lowest-observed-adverse-effect level; $n$, number; $P A L$, precautionary allergen labelling; $S D$, standard deviation. 
Supplemental Table 3. Domain scores of parent-proxy reported health-related quality of life using the FAQLQ-PF

\begin{tabular}{|c|c|c|c|c|c|}
\hline & & $\begin{array}{l}\text { Before } \\
\text { DBPCFC } \\
\text { (T1) }\end{array}$ & $\begin{array}{l}3 \text { years } \\
\text { after } \\
\text { DBPCFC } \\
\text { (T2) }\end{array}$ & $\begin{array}{l}\text { Delta } \\
\text { score }^{+}\end{array}$ & $\begin{array}{l}P \text { - } \\
\text { value }^{\ddagger}\end{array}$ \\
\hline & Domain & Mean (SD) & Mean (SD) & Mean & \\
\hline \multicolumn{6}{|l|}{ Parent-proxy reported HRQL } \\
\hline \multirow[t]{4}{*}{ All children $(n=44)$} & Overall & $2.38(0.97)$ & $2.17(1.00)$ & -0.22 & 0.187 \\
\hline & FRA & $2.66(1.17)$ & $2.53(1.18)$ & -0.14 & \\
\hline & SDL & $2.41(1.08)$ & $2.02(0.96)$ & -0.38 & \\
\hline & EI & $2.19(1.17)$ & $2.05(1.04)$ & -0.14 & \\
\hline \multirow[t]{4}{*}{ Peanut-allergic children $(n=23)$} & Overall & $2.29(1.02)$ & $2.35(0.80)$ & 0.05 & 0.823 \\
\hline & FRA & $2.50(1.22)$ & $2.66(0.80)$ & 0.16 & \\
\hline & SDL & $2.31(1.17)$ & $2.22(0.85)$ & -0.10 & \\
\hline & EI & $2.13(1.08)$ & $2.24(0.90)$ & 0.11 & \\
\hline \multirow[t]{4}{*}{ Peanut-tolerant children $(n=21)$} & Overall & $2.48(0.94)$ & $1.97(1.18)$ & -0.51 & 0.025 \\
\hline & FRA & $2.84(1.25)$ & $2.38(1.50)$ & -0.46 & \\
\hline & SDL & $2.50(1.00)$ & $1.80(1.05)$ & -0.69 & \\
\hline & EI & $2.25(1.29)$ & $1.84(1.15)$ & -0.40 & \\
\hline \multirow[t]{4}{*}{ Successful introduction $(n=15)$} & Overall & $2.59(1.06)$ & $1.90(1.20)$ & -0.70 & 0.013 \\
\hline & FRA & $2.89(1.38)$ & $2.22(1.35)$ & -0.67 & \\
\hline & SDL & $2.65(1.12)$ & $1.80(1.19)$ & -0.85 & \\
\hline & EI & $2.37(1.47)$ & $1.76(1.17)$ & -0.61 & \\
\hline \multirow[t]{4}{*}{ Failed introduction $(n=6)$} & Overall & $2.21(0.52)$ & $2.16(1.20)$ & -0.05 & 0.906 \\
\hline & FRA & $2.71(0.94)$ & $2.77(1.89)$ & 0.06 & \\
\hline & SDL & $2.11(0.51)$ & $1.81(0.71)$ & -0.30 & \\
\hline & EI & $1.94(0.72)$ & $2.04(1.18)$ & 0.10 & \\
\hline \multicolumn{6}{|l|}{ Child-reported HRQL } \\
\hline Child Form $(n=6)$ & Overall & $3.72(1.82)$ & $3.29(1.72)$ & -0.42 & 0.648 \\
\hline Teen Form $(n=3)$ & Overall & $4.54(1.15)$ & $3.85(1.26)$ & -0.69 & 0.270 \\
\hline Child Form (T1) + Teen Form (T2) $(n=14)$ & Overall & $3.48(1.26)$ & $2.85(1.14)$ & NA & NA \\
\hline None $(T 1)+$ Child Form $(T 2)(n=18)$ & Overall & NA & $3.40(1.75)$ & NA & NA \\
\hline
\end{tabular}




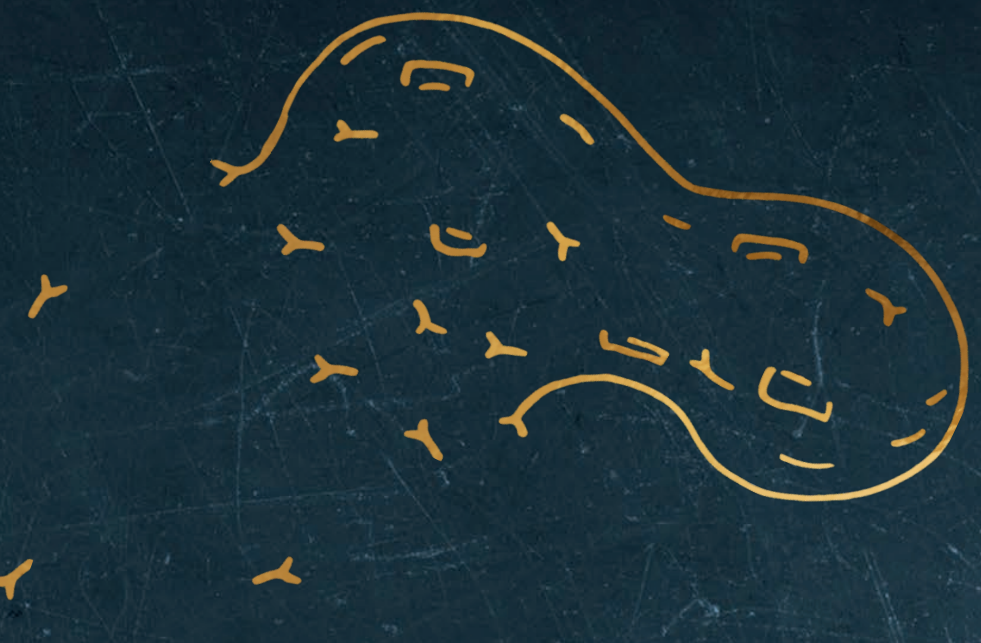

$+$

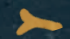




\section{Chapter 5}

\section{Risk factors for atopic diseases and recurrent respiratory tract infections in children}




\section{Abstract}

\section{Background}

The simultaneous increased prevalence of atopic diseases and decreased prevalence of infectious diseases might point to a link between the two entities. Past work mainly focused on either atopic diseases or recurrent infections. We aim to investigate whether demographic, environmental and family history risk factors for atopic diseases (i.e. asthma, allergic rhinitis, atopic dermatitis and/or food allergy) differ from risk factors for recurrent respiratory tract infections (RRTI) in children.

\section{Methods}

Cross-sectional data were used from 5517 children aged 1-18 years who participated in an Electronic Portal for children with asthma, allergies or infections between 2011 and 2019. Univariable/multivariable logistic regression analyses were performed to determine risk factors for any atopic disease and RRTI.

\section{Results}

Children aged $\geq 5$ years were more likely to have any atopic disease (adjusted OR, $\mathrm{OR}, 1.50-2.77$ ) and less likely to have RRTI (adjusted OR, 0.68-0.84) compared to children aged < 5 years. Female sex (adjusted OR 0.72; 95\% CI 0.63-0.81), low birth weight (adjusted OR 0.74; 95\% CI 0.57-0.97) and dog ownership (adjusted OR 0.79; 95\% CI 0.66-0.95) reduced the odds of any atopic disease, but not of RRTI. Day care attendance (adjusted OR 1.22; 95\% CI 1.02-1.47) was associated with RRTI, but not with atopic diseases. A family history of asthma, allergic rhinitis, atopic dermatitis and RRTI was significantly associated with the same entity in children, with an adjusted OR varying from 1.58 (95\% CI 1.35-1.85) in allergic rhinitis to 2.20 (95\% CI 1.85-2.61) in asthma.

\section{Conclusion}

Risk factors for atopic diseases are distinct from risk factors for RRTI, suggesting that the changing prevalence of both entities is not related to shared risk factors. 


\section{Introduction}

Atopic diseases are common diseases in childhood. The prevalence of asthma, allergic rhinitis, atopic dermatitis and food allergy has been estimated at approximately $20 \%, 15 \%, 8 \%$ and $6 \%$, respectively. ${ }^{1,2}$ The prevalence of atopic diseases has been steadily rising in the past decades in the Western world. ${ }^{3}$ Simultaneously, the prevalence of infectious diseases has decreased. ${ }^{4}$ These two trends in prevalence have been linked, leading to the hypothesis that a decline in infections or a lack of exposure to microbes could have an etiological role in the increased prevalence of atopic diseases. The known geographical variation in the prevalence of atopic diseases is in line with this hypothesis: a high prevalence in industrialized, urban environments concurrent with low microbial exposure, and a low prevalence in the developing or rural world concurrent with high microbial exposure. ${ }^{5}$ It is known that early, repeated exposure to infectious agents stimulates the immune system to develop regulatory T-cells, and hereby the development of atopic diseases may be prevented. ${ }^{6}$

Environmental factors associated with a reduced risk of atopic diseases are often described as being associated with an increased risk of infections, such as day care attendance, growing up in a rural environment, having older siblings and having pets. ${ }^{7-10}$ A large number of epidemiological studies have described that other environmental factors, such as maternal smoking during pregnancy, postnatal exposure to cigarette smoke, air pollution and a family history of atopic diseases, are associated with an increased prevalence of atopic diseases. ${ }^{11}$

Although the opposing trends in the prevalence of atopic diseases and infections have been linked, studies investigating demographic, environmental and family history risk factors for both atopic diseases and recurrent infections in conjunction are lacking. Large studies investigating a broad range of risk factors for atopic diseases and recurrent infections that enable adjustment for each other may lead to a better understanding of the development of these disease entities in children. A large set of data on this topic is available in the so-called Electronic Portal developed by the UMC Utrecht. This is a standardized data collection tool used in multiple centres across the Netherlands that includes data of several validated questionnaires on atopic diseases and recurrent respiratory tract infections in childhood. ${ }^{12}$ The primary aim of this study was to investigate how risk factors associated with any atopic disease (i.e. asthma, allergic rhinitis, atopic dermatitis and/or food allergy) are different from risk factors associated with recurrent respiratory tract infections (RRTI) in children. Our secondary aim was to investigate how these risk factors are associated with individual atopic disease entities. 


\section{Methods}

\section{Domain and data collection}

We performed a cross-sectional questionnaire-based study among children aged 1 to 18 years old who participated in the Electronic Portal between June 2011 and September 2019. The Electronic Portal is a web-based application established by a nationwide collaborative network of Dutch caregivers. The details of the Electronic Portal have been previously published. ${ }^{12}$ Children were included in the Electronic Portal as part of a first outpatient visit for respiratory or allergic symptoms in a participating secondary care $(n=9)$ or tertiary care $(n=1)$ centre or as part of the WHISTLER birth cohort study. ${ }^{13}$ No exclusion criteria were specified. Of the 9,558 children who were invited to participate in the Electronic Portal, 5517 (58\%) children started the questionnaire and were evaluated in this study. Informed consent was obtained from all parents and/or children (as applicable) before enrolment. The study was reviewed and approved by the medical ethics committee of the University Medical Centre Utrecht (No. 10/348).

\section{Outcome definitions}

Information on the presence of atopic diseases and RRTI was extracted from the answers to the questionnaires in the Electronic Portal (Supplemental Table 1). The definition of asthma, allergic rhinitis and atopic dermatitis was adopted from the ISAAC questionnaires. ${ }^{14}$ Food allergy was defined as suggestive allergic symptoms within 2 hours after ingestion of the suspected food. Recurrent respiratory tract infections (RRTI) was defined as having a minimum number of upper or lower respiratory infection per year. ${ }^{15}$ The minimum number depended on the child's age (Supplemental Table 1).

\section{Demographic, environmental and family history factors}

All risk factors were selected based on prior research and clinical expertise of the authors. ${ }^{7-11}$ Information on the presence of the selected risk factors was extracted from answers to the questionnaires in the Electronic Portal. Included demographic factors were age, sex, gestational age, birth weight, maternal and paternal ethnicity and education level. Included environmental factors were exclusive breastfeeding, pacifier use, having siblings, number of older siblings, day care attendance in the first year of life, living environment (i.e. rural or urban), having a dog, having a cat, maternal smoking during pregnancy, indoor smoking, adoption, vaccination status (i.e. complete age-appropriate vaccinations according to the Dutch vaccination schedule), flooring in the house and flooring in the child's bedroom (i.e. solid or carpet). Included factors on family history were having a family history (i.e. one or both parents and/or sibling(s) with the disease) of asthma, allergic rhinitis, atopic dermatitis, food allergy or recurrent respiratory tract infections. 


\section{Statistical analysis}

Descriptive statistics including numbers (percentages) and medians (interquartile range) were used to describe the study population. Data on all risk factors and outcomes were complete in $55 \%$ of the children and $92 \%$ of the values. Missing values were imputed by using multiple imputations ( 20 iterations) using SPSS. First, the crude associations between risk factors and the disease outcome (i.e. any of the atopic diseases or RRTI) were assessed using univariable logistic regression analyses. Second, the adjusted associations between the risk factors and outcome were assessed using multivariable logistic regression analyses. The multivariable regression analyses included all risk factors (no prior selection based on p-values), the child's comorbidity and the analyses were controlled for inclusion setting (i.e. secondary care, tertiary care or birth cohort). The same analyses were performed per individual atopic disease entity. Results of the univariable and multivariable regression analyses were expressed as respectively crude odds ratios and adjusted odds ratios (OR) with $95 \%$ confidence intervals $(95 \% \mathrm{CI})$. A p-value of $<0.05$ was considered statistically significant. Data were analysed using SPSS version 25.0 for Windows (SPPS, INC, Chicago, IL, USA).

\section{Results}

\section{Patients}

The median age of the 5,517 included children was 6 years (interquartile range 3 to 10 ) and $43 \%$ were girls (Table 1 ). Children were included after referral to a secondary care centre (56\%), a tertiary care centre (30\%) or as part of the WHISTLER birth cohort (14\%). Sixty-two percent of children had one or more atopic disease(s), $42 \%$ had recurrent respiratory tract infections (RRTI) and $23 \%$ had no atopic diseases or RRTI (Figure 1). 
Table 1. Baseline characteristics of the cohort $(n=5517)$

n (\%)

\begin{tabular}{|c|c|c|}
\hline \multicolumn{3}{|l|}{ Demographics } \\
\hline Age in years, median (IQR) & & $6.00(3-10)$ \\
\hline Female sex & & $2370(43)$ \\
\hline \multirow{3}{*}{ Gestational age } & $<37$ weeks & $607(11)$ \\
\hline & 37-43 weeks & $4807(87)$ \\
\hline & $\geq 43$ weeks & $103(2)$ \\
\hline Low birth weight & & $429(8)$ \\
\hline Dutch maternal ethnicity & & $5116(93)$ \\
\hline Dutch paternal ethnicity & & $4999(91)$ \\
\hline \multirow[t]{3}{*}{ Maternal education level } & Low & $528(10)$ \\
\hline & Middle & $1888(34)$ \\
\hline & High & $3101(56)$ \\
\hline \multirow[t]{3}{*}{ Paternal education level } & Low & $612(11)$ \\
\hline & Middle & $1944(35)$ \\
\hline & High & $2961(54)$ \\
\hline \multicolumn{3}{|l|}{ Environment } \\
\hline \multirow[t]{3}{*}{ Exclusive breastfeeding } & Never & $3583(65)$ \\
\hline & $<6$ months & $948(17)$ \\
\hline & $\geq 6$ months & $987(18)$ \\
\hline Pacifier use & & $3600(65)$ \\
\hline Having siblings & & $4349(79)$ \\
\hline Day care attendance & & $4688(85)$ \\
\hline Urban living environment & & $2360(43)$ \\
\hline Having a pet dog & & $972(18)$ \\
\hline Having a pet cat & & $971(18)$ \\
\hline Maternal smoking during pregnancy & & $221(4)$ \\
\hline Indoor smoking & & $287(5)$ \\
\hline Adopted & & $71(1)$ \\
\hline Vaccinated & & $4946(90)$ \\
\hline \multicolumn{3}{|l|}{ Family history } \\
\hline Asthma & & $2230(41)$ \\
\hline Allergic rhinitis & & $3610(65)$ \\
\hline Atopic dermatitis & & $3312(60)$ \\
\hline Food allergy & & $1926(35)$ \\
\hline RRTI & & $3103(56)$ \\
\hline \multicolumn{3}{|l|}{ Disease outcome child } \\
\hline Asthma & & $958(17)$ \\
\hline Allergic rhinitis & & $1919(35)$ \\
\hline Atopic dermatitis & & $2083(38)$ \\
\hline Food allergy & & $552(10)$ \\
\hline RRTI & & $2336(42)$ \\
\hline \multicolumn{3}{|l|}{ Combined disease outcome child } \\
\hline Any atopic disease & & $3408(62)$ \\
\hline Both any atopic disease and RRTI & & $1523(28)$ \\
\hline No atopic disease or RRTI & & $1295(23)$ \\
\hline
\end{tabular}

Percentages do not always add up to 100 due to rounding.

Abbreviations: IQR, interquartile range; $n$, number; RRTI, recurrent respiratory tract infections. 

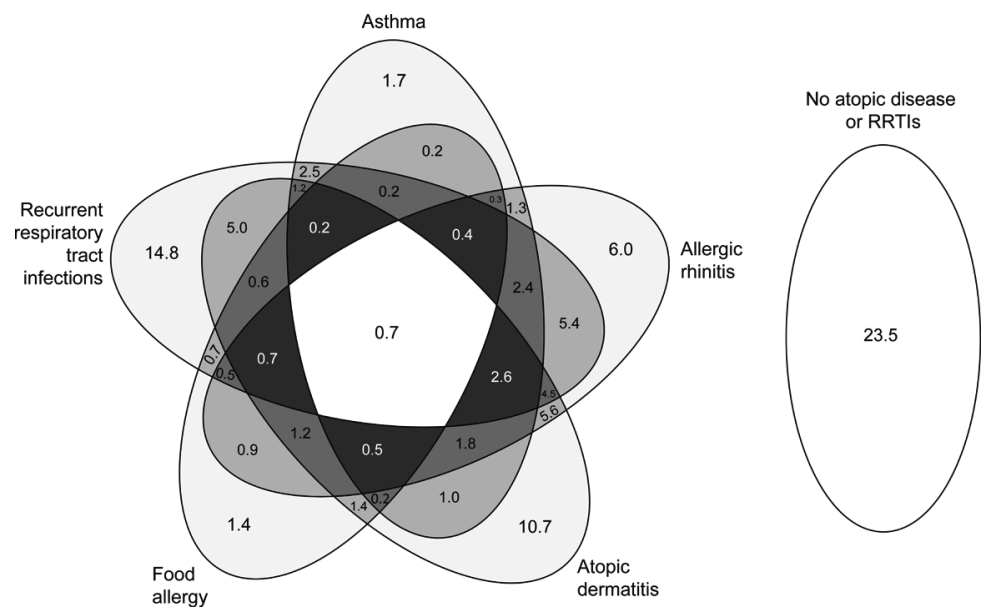

Figure 1. Venn diagram displaying the proportion of atopic diseases and respiratory tract infections in all included children

\section{Risk factors for atopic diseases and recurrent respiratory tract infections}

Table 2 and Figure 2 report the results from the multivariable regression analyses, exploring the association between demographic, environmental and family history risk factors and the presence of any atopic disease or RRTI. Table 3 reports the results from the multivariable regression analyses per individual atopic disease entity. The results of the univariable analyses are presented in Supplemental Table 2 and 3.

\section{Demographic factors}

Several demographic factors were associated with any atopic disease, while only age and gestational age were associated with RRTI (Table 2; Figure 2A). Children aged 5 years and older had a higher chance of any atopic disease (adjusted Odds Ratio, OR, 1.50 to 2.77), but a lower chance of RRTI (adjusted OR, 0.68 to 0.84 ), compared to children aged $<5$ years. Female sex (adjusted OR, 0.72; 95\% CI, 0.63-0.81) and low birth weight (adjusted OR, 0.74; 95\% CI, 0.57-0.97) were associated with a reduced odds of any atopic disease. Children born between 37 and 43 weeks of gestation had a lower chance of RRTI (adjusted OR, 0.79; 95\% CI, 0.62-0.99), compared to children born preterm.

In the analyses per individual atopic disease entity, children aged 5 years and older had a higher chance of asthma (adjusted OR, 3.07 to 5.21) and allergic rhinitis (adjusted OR, 2.20 to 6.65) compared to children $<5$ years (Table 3). Children aged 5 years and older had a lower chance of atopic dermatitis (adjusted OR, 0.44 to 0.77 ) compared to children aged $<5$ years. Female sex was associated with a reduced odds of asthma (adjusted OR, 0.83; 95\% CI, 0.70-0.97) and allergic rhinitis (adjusted OR, $0.70 ; 95 \% \mathrm{CI}, 0.61-0.81)$. Low birth weight was associated with a reduced odds of allergic rhinitis (adjusted OR, 0.72; 95\% CI, 0.54-0.96) only. 
Table 2. Independent risk factors for respectively any atopic disease and RRTI

\begin{tabular}{|c|c|c|c|c|}
\hline & \multicolumn{2}{|c|}{ Any atopic disease } & \multicolumn{2}{|l|}{ RRTI } \\
\hline & $\begin{array}{l}\text { Adjusted OR } \\
(95 \% \mathrm{CI})\end{array}$ & $P$ value $^{+}$ & $\begin{array}{l}\text { Adjusted OR } \\
(95 \% \text { CI) }\end{array}$ & $P$ value $^{+}$ \\
\hline \multicolumn{5}{|l|}{ Demographics } \\
\hline \multicolumn{5}{|l|}{ Age (in years) } \\
\hline 5 to 8 (vs. 1 to 4$)$ & $1.50(1.28-1.75)$ & $<0.001$ & $0.84(0.71-1.01)$ & 0.06 \\
\hline 9 to 12 (vs. 1 to 4 ) & $2.04(1.71-2.44)$ & $<0.001$ & $0.69(0.57-0.83)$ & $<0.001$ \\
\hline 13 to 17 (vs. 1 to 4 ) & $2.77(2.20-3.50)$ & $<0.001$ & $0.68(0.53-0.86)$ & $<0.01$ \\
\hline Female sex & $0.72(0.63-0.81)$ & $<0.001$ & $1.04(0.91-1.19)$ & 0.54 \\
\hline \multicolumn{5}{|l|}{ Gestational age (weeks) } \\
\hline 37-43 weeks (vs. < 37) & $0.98(0.78-1.22)$ & 0.84 & $0.79(0.62-0.99)$ & $<0.05$ \\
\hline$\geq 43$ weeks $($ vs. $<37)$ & $0.91(0.54-1.54)$ & 0.72 & $0.68(0.40-1.15)$ & 0.15 \\
\hline Low birth weight (vs. normal) & $0.74(0.57-0.97)$ & 0.03 & $0.90(0.69-1.17)$ & 0.44 \\
\hline Non-Dutch mother (vs. Dutch) & $0.93(0.68-1.26)$ & 0.63 & $0.99(0.71-1.37)$ & 0.95 \\
\hline Non-Dutch father (vs. Dutch) & $1.43(1.10-1.87)$ & $<0.01$ & $1.03(0.80-1.33)$ & 0.80 \\
\hline \multicolumn{5}{|l|}{ Education level mother } \\
\hline Middle (vs. low) & $1.35(1.05-1.74)$ & 0.02 & $0.83(0.65-1.07)$ & 0.16 \\
\hline High (vs. low) & $1.27(0.98-1.66)$ & 0.08 & $0.78(0.59-1.03)$ & 0.08 \\
\hline \multicolumn{5}{|l|}{ Education level father } \\
\hline Middle (vs. low) & $1.02(0.82-1.29)$ & 0.84 & $0.85(0.68-1.07)$ & 0.16 \\
\hline High (vs. low) & $1.03(0.81-1.30)$ & 0.82 & $0.83(0.65-1.06)$ & 0.14 \\
\hline \multicolumn{5}{|l|}{ Environment } \\
\hline \multicolumn{5}{|l|}{ Exclusive breastfeeding } \\
\hline$<6$ months (vs. never) & $1.02(0.85-1.21)$ & 0.87 & $0.86(0.72-1.03)$ & 0.10 \\
\hline$\geq 6$ months (vs. never) & $1.08(0.90-1.28)$ & 0.41 & $0.96(0.81-1.13)$ & 0.61 \\
\hline Pacifier use & $1.04(0.91-1.19)$ & 0.57 & $1.10(0.96-1.25)$ & 0.17 \\
\hline Having siblings & $0.88(0.72-1.09)$ & 0.25 & $1.01(0.79-1.29)$ & 0.92 \\
\hline \multicolumn{5}{|l|}{ Number of siblings } \\
\hline 1 (vs. 0) & $0.94(0.80-1.11)$ & 0.48 & $1.01(0.86-1.19)$ & 0.89 \\
\hline 2 (vs. 0) & $0.81(0.64-1.04)$ & 0.10 & $1.12(0.88-1.43)$ & 0.36 \\
\hline$\geq 3$ (vs. 0) & $0.77(0.40-1.45)$ & 0.41 & $0.79(0.49-1.28)$ & 0.34 \\
\hline Day care attendance & $1.11(0.93-1.34)$ & 0.24 & $1.22(1.02-1.47)$ & $<0.05$ \\
\hline Urban living environment (vs. rural) & $0.97(0.85-1.11)$ & 0.63 & $0.92(0.81-1.05)$ & 0.23 \\
\hline Having a pet dog & $0.79(0.66-0.95)$ & 0.01 & $1.24(1.04-1.48)$ & $<0.05$ \\
\hline Having a pet cat & $0.85(0.72-1.00)$ & 0.05 & $1.09(0.92-1.28)$ & 0.34 \\
\hline Maternal smoking during pregnancy & $1.01(0.72-1.42)$ & 0.96 & $1.14(0.80-1.62)$ & 0.47 \\
\hline Indoor smoking & $1.27(0.92-1.77)$ & 0.15 & $1.20(0.88-1.63)$ & 0.26 \\
\hline Adopted & $1.65(0.91-3.00)$ & 0.10 & $0.53(0.28-1.01)$ & 0.05 \\
\hline Vaccinated & $0.94(0.76-1.16)$ & 0.56 & $1.15(0.93-1.42)$ & 0.19 \\
\hline Carpet floor house (vs. solid) & $1.96(1.10-3.48)$ & 0.02 & $0.89(0.55-1.44)$ & 0.64 \\
\hline Carpet floor bedroom child (vs. solid) & $0.82(0.69-0.98)$ & 0.03 & $0.95(0.79-1.13)$ & 0.55 \\
\hline \multicolumn{5}{|l|}{ Family history } \\
\hline Asthma & $1.24(1.07-1.42)$ & $<0.01$ & $1.03(0.90-1.18)$ & 0.70 \\
\hline Allergic rhinitis & $1.30(1.13-1.50)$ & $<0.001$ & $0.91(0.78-1.06)$ & 0.23 \\
\hline Atopic dermatitis & $1.66(1.45-1.90)$ & $<0.001$ & $1.03(0.90-1.19)$ & 0.64 \\
\hline Food allergy & $1.29(1.09-1.52)$ & $<0.01$ & $0.91(0.79-1.06)$ & 0.23 \\
\hline RRTI & $0.91(0.80-1.04)$ & 0.18 & $1.71(1.50-1.96)$ & $<0.001$ \\
\hline
\end{tabular}


Table 2. Independent risk factors for respectively any atopic disease and RRTI (continued)

\begin{tabular}{lllll}
\hline & $\begin{array}{l}\text { Any atopic disease } \\
\text { Adjusted OR } \\
(95 \% \mathrm{CI})\end{array}$ & $P$ value $^{+}$ & $\begin{array}{l}\text { RRTI } \\
\text { Adjusted OR } \\
(95 \% \mathrm{CI})\end{array}$ & $P$ value $^{+}$ \\
\hline Comorbidities & & & & \\
Asthma & NA & $2.01(1.68-2.39)$ & $<\mathbf{0 . 0 0 1}$ \\
Allergic rhinitis & NA & $1.39(1.19-1.63)$ & $<\mathbf{0 . 0 0 1}$ \\
Atopic dermatitis & NA & $0.70(0.61-0.81)$ & $<\mathbf{0 . 0 0 1}$ \\
Food allergy & NA & & $0.62(0.49-0.77)$ & $<\mathbf{0 . 0 0 1}$ \\
RRTI & $0.94(0.82-1.09)$ & 0.44 & NA & \\
\hline
\end{tabular}

Analyses were mutually adjusted within the model, for centre (secondary care, tertiary care or birth cohort) and for the child's comorbidities. Significant $\mathrm{P}$ values are in bold.

Abbreviations: $\mathrm{Cl}$, confidence interval; $O R$, odds ratio; $R R T I$, recurrent respiratory tract infections. 
Chapter 5

\section{Demographics}

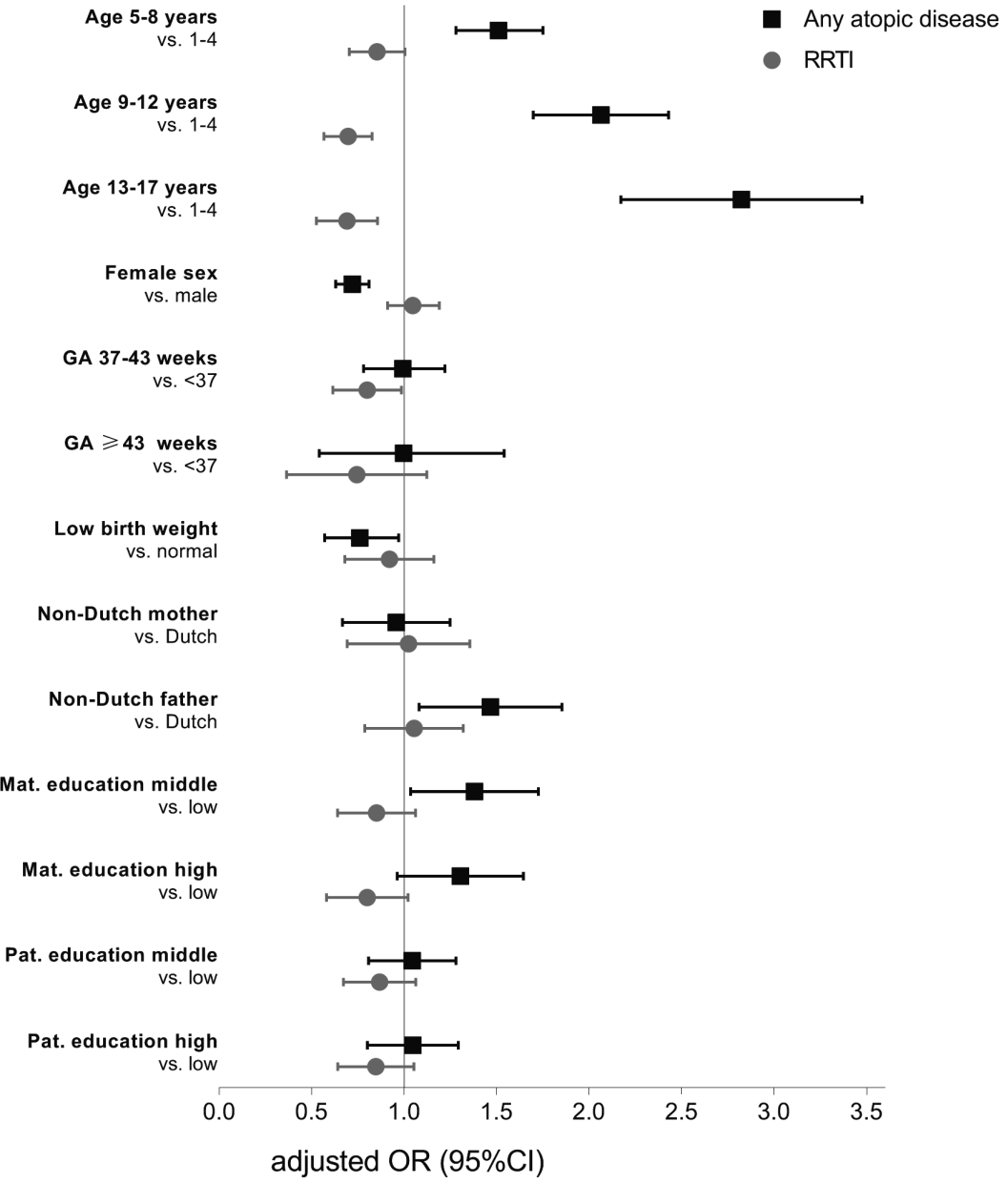

Figure 2A. Association between demographic risk factors and any atopic disease and RRTI Abbreviations: GA, gestational age; mat, maternal; OR, odds ratio; pat, paternal; RRTI, recurrent respiratory tract infections. 


\section{Environment}

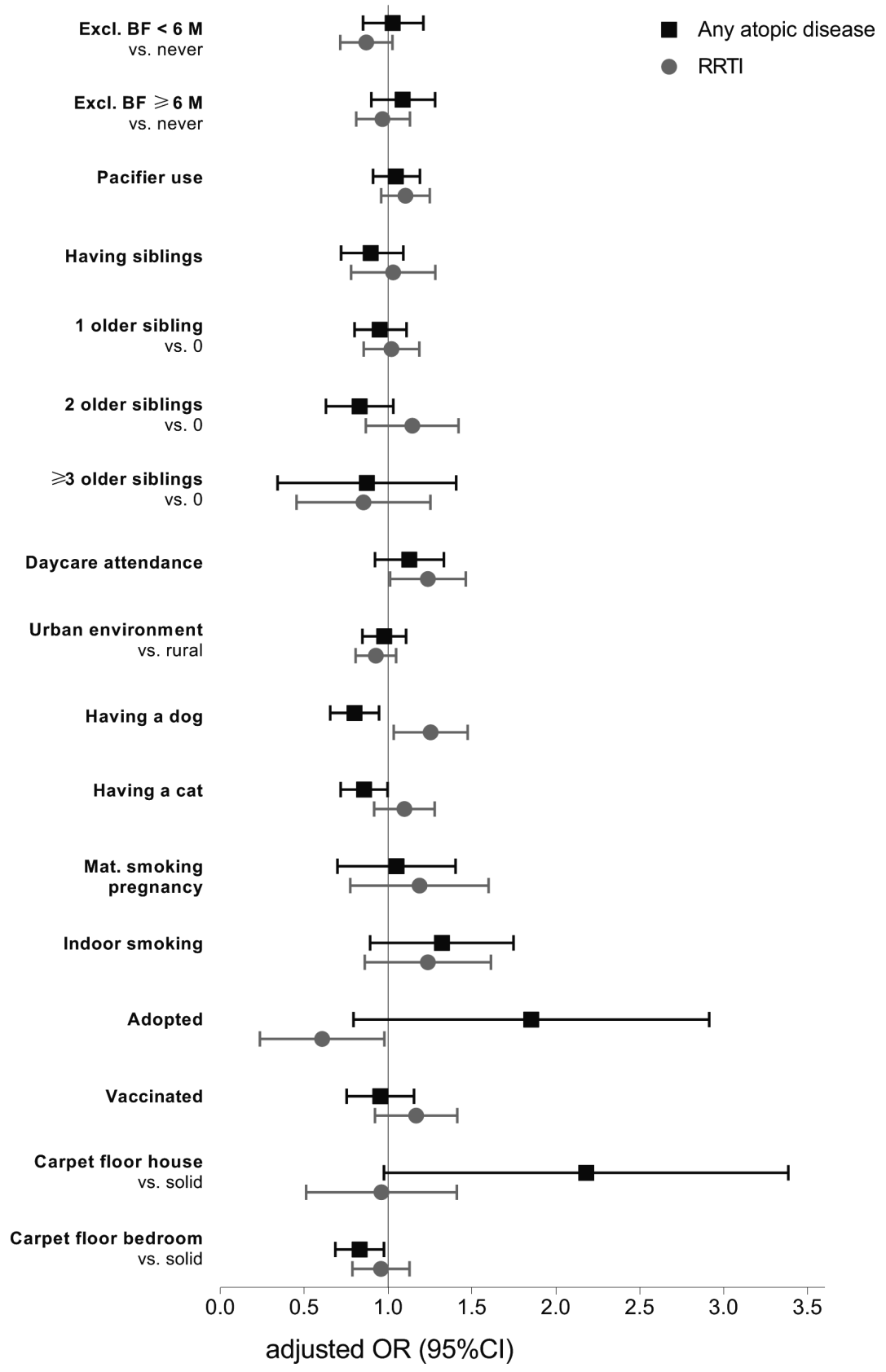

Figure 2B. Association between environmental risk factors and any atopic disease and RRTI Abbreviations: excl. $B F$, exclusive breastfeeding; mat, maternal; OR, odds ratio; RRTI, recurrent respiratory tract infections. 

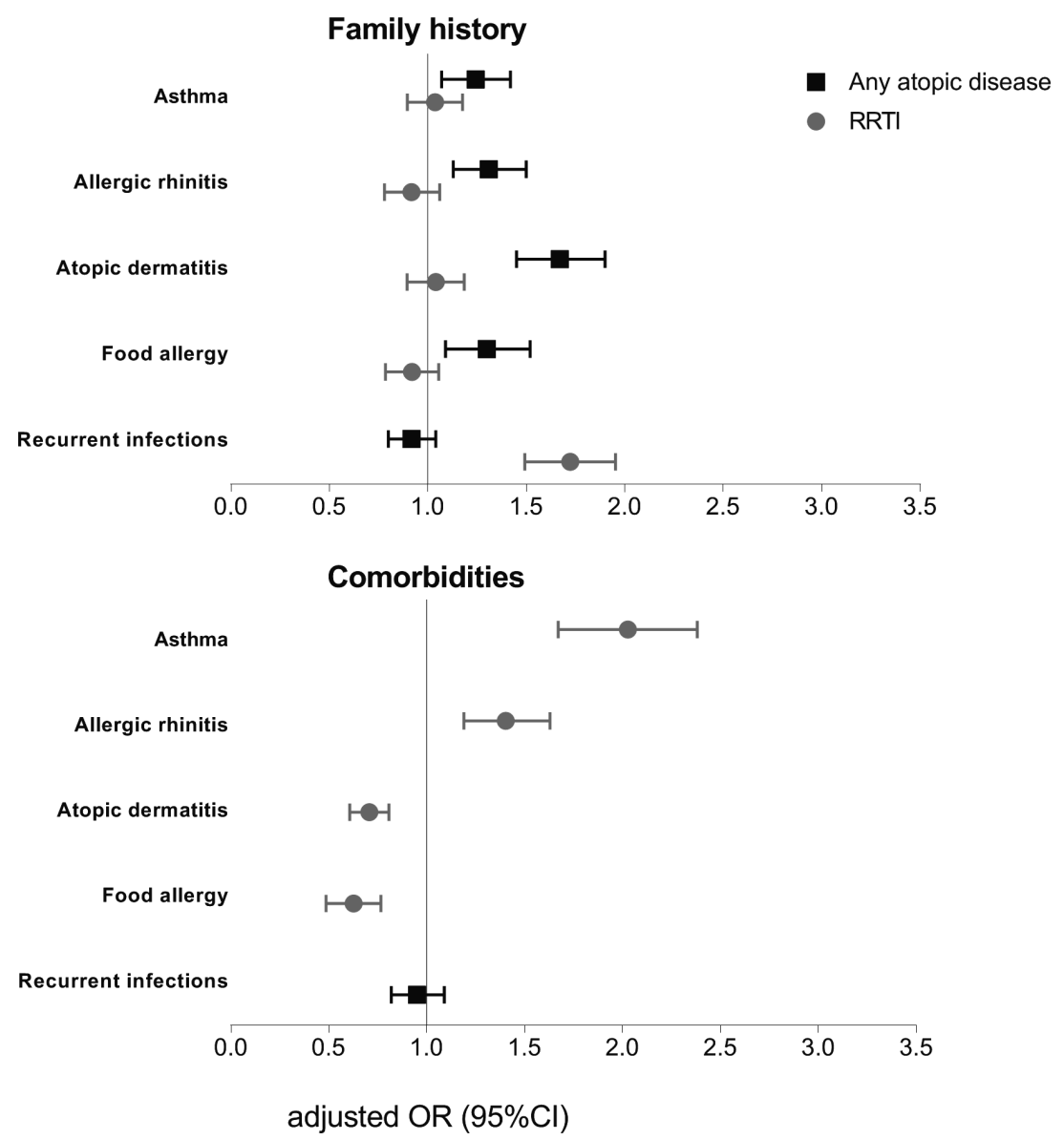

Figure $\mathbf{2 C}$. Association between family history and comorbidity risk factors and any atopic disease and RRTI

Abbreviations: $O R$, odds ratio; RRTI, recurrent respiratory tract infections.

\section{Environmental factors}

Children who had a pet dog (adjusted OR, 0.79; 95\% CI, 0.66-0.95) had a lower chance of any atopic disease, but a slightly higher chance of RRTI (adjusted OR, 1.24; $95 \%$ $\mathrm{CI}, 1.04-1.48$ ). Day care attendance increased the odds of RRTI (adjusted OR, 1.22; $95 \% \mathrm{CI}, 1.02-1.47$ ), but was not associated with any atopic disease (Table 2; Figure 2B).

In the analyses per individual atopic disease entity, children who had a pet dog had a lower chance of food allergy (adjusted OR, 0.52; 95\% CI, 0.38-0.72) (Table 3). Furthermore, children who had a pet cat had a lower chance of allergic rhinitis (adjusted OR, $0.71 ; 95 \% \mathrm{CI}, 0.59-0.87$ ). In addition, children with 1 or 2 siblings had a lower chance of allergic rhinitis (adjusted OR, 0.84; 95\% CI, 0.71-0.99 and adjusted $\mathrm{OR}, 0.71 ; 95 \%$ CI 0.55-0.91, respectively). Children who were exclusively breastfed for 
6 months or longer had a higher chance of food allergy (adjusted OR, 1.34; $95 \% \mathrm{CI}$, 1.04-1.72). An unexpected finding was that we observed an inverse association between maternal smoking during pregnancy and atopic dermatitis (adjusted OR $0.57 ; 95 \%$ CI 0.40-0.82).

\section{Family history}

The associations between family history and disease outcomes were disease-specific. Children who reported a family history of one of the atopic diseases were more likely to have any atopic disease, but were not likely to have more RRTI (Table 2; Figure 2C). Children who reported a family history of recurrent infections were more likely to have RRTI (adjusted OR, 1.71; $95 \%$ CI, 1.50-1.96), but were not more likely to have any atopic disease.

In the analyses per individual atopic disease entity, the disease-specific association between a family history and disease outcome was further confirmed for asthma, allergic rhinitis and atopic dermatitis (adjusted OR 2.20, 1.58, 1.72, respectively).

\section{Comorbidities}

A child's history of RRTI was not associated with having any atopic disease (adjusted OR, 0.94; 95\% CI, 0.82-1.09) (Table 2; Figure 2C). However, children who reported a history of asthma or allergic rhinitis were more likely to have RRTI (adjusted OR, 2.01; 95\% CI, 1.68-2.39, and adjusted OR, 1.39; 95\% CI, 1.19-1.63, respectively). On the contrary, a history of atopic dermatitis or food allergy reduced the odds of having RRTI (adjusted OR, 0.70; 95\% CI, 0.61-0.81, and adjusted OR, $0.62,95 \%$ CI $0.49-0.77)$.

In the analysis per individual atopic disease entity, a child's history of another atopic disease increased the odds of the outcome atopic disease (adjusted OR ranging between 1.34 and 2.11) (Table 3). Although a child's history of RRTI was not associated with having any atopic diseases, a history of RRTI was associated with individual atopic diseases entities. Children who reported RRTI were more likely to have asthma (adjusted OR, 1.99; 95\% CI, 1.67-2.37) and allergic rhinitis (adjusted $\mathrm{OR}, 1.38 ; 95 \% \mathrm{CI}, 1.18-1.61$ ), but less likely to have atopic dermatitis (adjusted OR, $0.70 ; 95 \% \mathrm{CI}, 0.61-0.81$ ) and food allergy (adjusted OR, 0.63; 95\% CI, 0.50-0.80). 


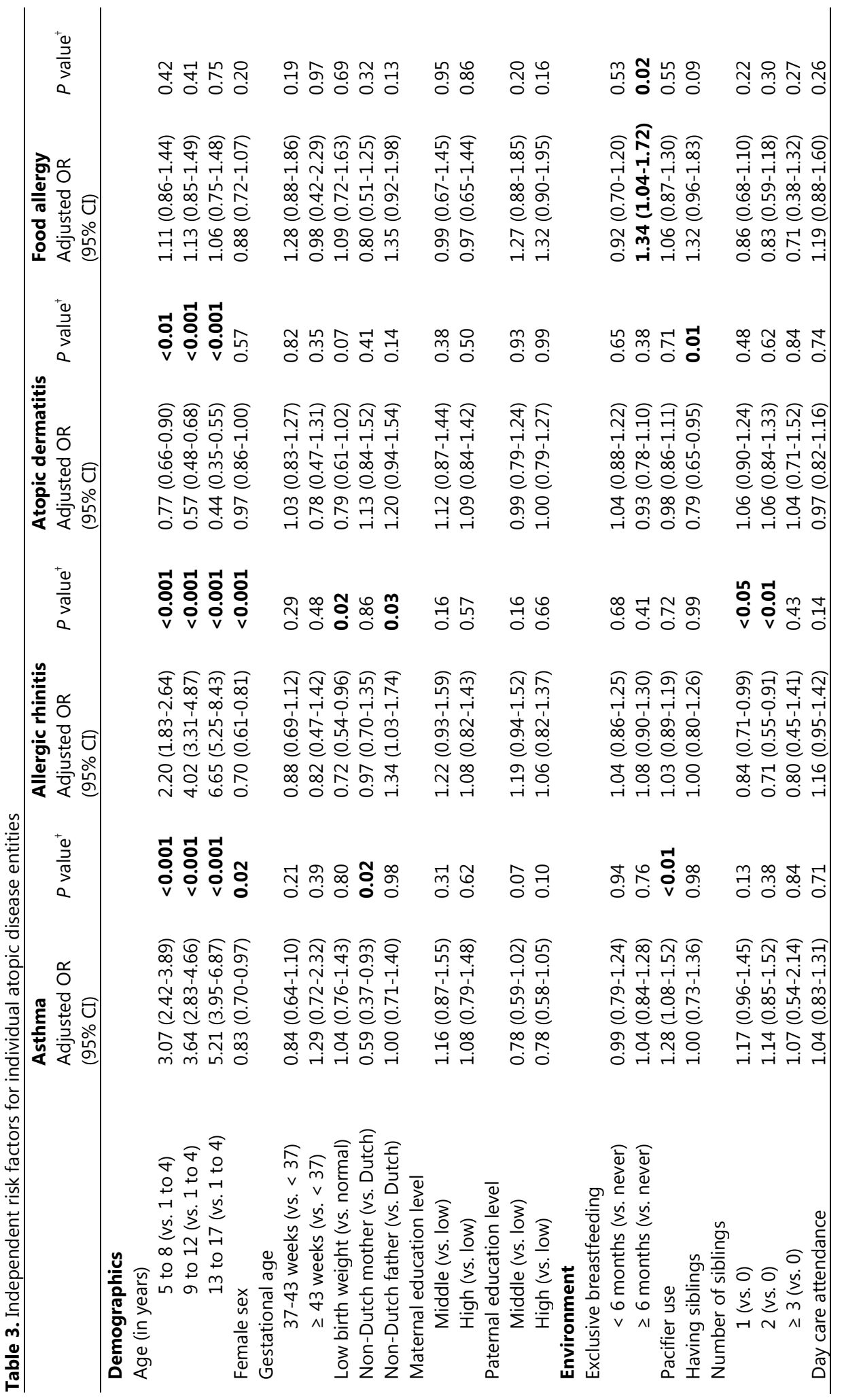




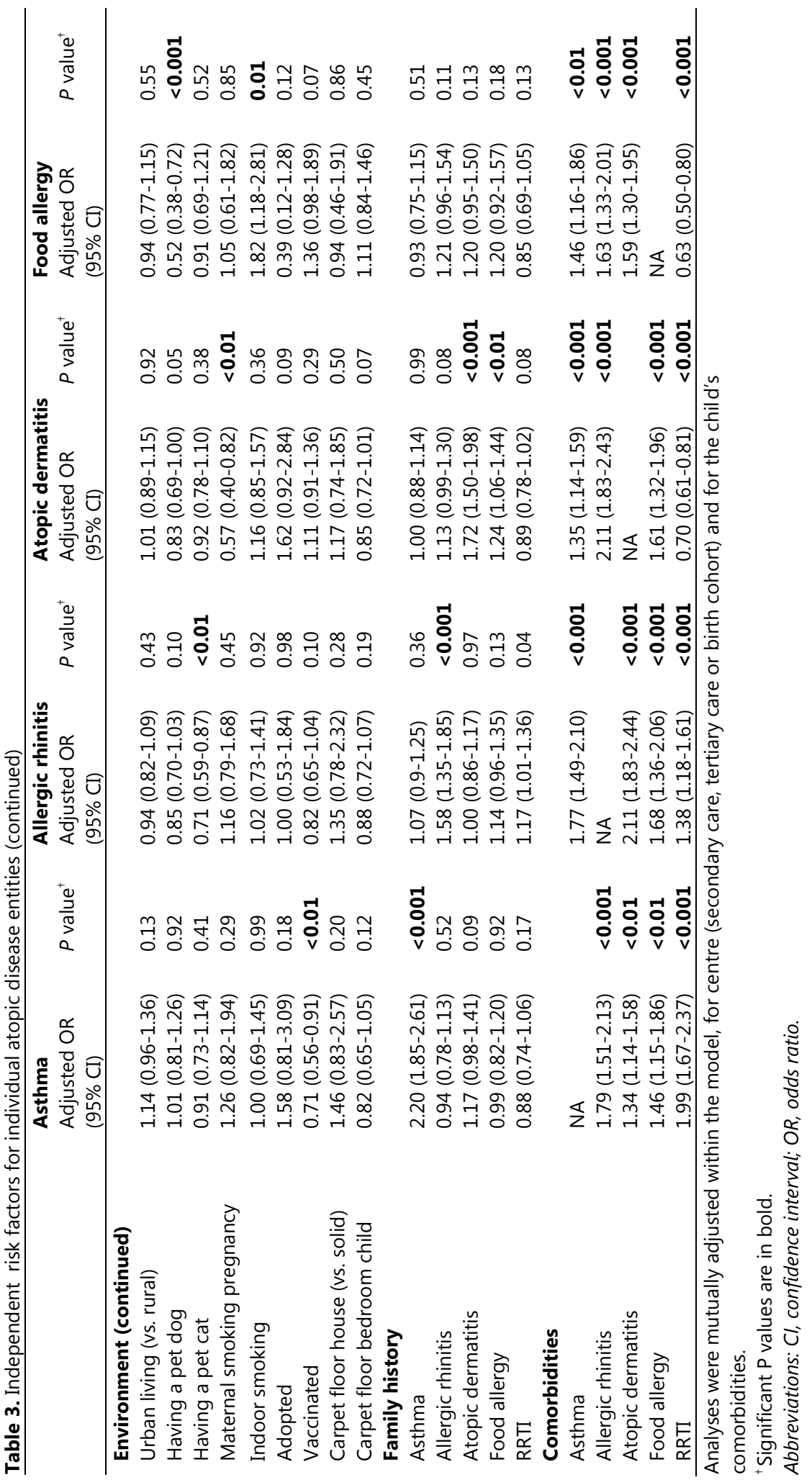




\section{Discussion}

Here, we present an overview of demographic, environmental and family history risk factors for atopic diseases compared to risk factors for recurrent respiratory tract infections (RRTI) in a large cohort of Dutch children. Our data show that risk factors for atopic diseases and RRTI differ. As an example, girls, children with a low birthweight and children with a pet dog were more likely to have atopic diseases, but were not more likely to have RRTI. Furthermore, a corresponding family history was a disease-specific risk factor for atopic diseases and for RRTI.

Children aged 5 years and older had a higher chance of asthma and allergic rhinitis compared to children younger than 5 years, while older children had a lower chance of atopic dermatitis. These findings are consistent with the so called 'atopic march'. ${ }^{16}$ The atopic march is thought to start with atopic dermatitis, followed by food allergy, asthma and allergic rhinitis. Furthermore, female sex lowered the likelihood of atopic diseases as has been previously acknowledged. ${ }^{17}$ Epidemiological studies have reported a predominance of atopic diseases in males before puberty and in females after puberty, possibly explained by hormonal influences or sex-specific genetic, environmental or social factors. ${ }^{18}$ We did not observe an association between sex and RRTI, although previous studies suggest that males are more susceptible to most types of respiratory tract infections. ${ }^{19}$ The possible protective effect of low birthweight on atopic disease has been previously reported ${ }^{20}$, and may be explained by differences in immune system development, gastrointestinal tract permeability and exposure to antigens between children with low and normal birthweight.

We found an increased risk of food allergy in children who were exclusively breastfed for more than 6 months. These children may have introduced allergenic foods later in life. Our data consolidate existing evidence that early introduction of allergenic foods prevents the development of food allergies. ${ }^{21}$ Day care attendance increased the risk of RRTI as has been acknowledged in previous research. ${ }^{9}$ The finding that dog ownership was associated with a reduced risk of atopic diseases confirms evidence that having a pet dog protects against atopic diseases and sensitization. ${ }^{7}$ The possible protective effect of pet ownership might be explained by an immunomodulatory effect of exposure to allergens, endotoxins or bacteria ${ }^{7}$ or by affecting DNA methylation. ${ }^{22}$ However, the protective effect of pet ownership may be partly attributed to selective avoidance of pets in households with allergic family members (reverse causality). ${ }^{23}$

The association between family history and the child's outcome was disease-specific, i.e. children with asthma, allergic rhinitis, atopic dermatitis or RRTI were significantly more likely to have a parent and/or sibling reporting the same condition. Especially 
the association between family history and RRTI is intriguing and requires further investigation, as it has not been described before. A family history of RRTI is often overlooked although it is particularly important to identify children at increased risk of RRTI and children with genetic disorders or immune deficiencies. ${ }^{24}$ Given the rarity of single-gene immunodeficiency diseases ${ }^{25}$, it is suggestive that this association can be more readily explained by a polygenic inheritance pattern. Host genetic factors have been implicated in respiratory infections of varying aetiology, but no consistent associations are observed. ${ }^{26}$ Most likely, gene-environment interactions play a role in the pathogenesis of both atopic diseases and RRTI. Regardless of the aetiology of the observed association between family history and disease phenotype, our results do emphasize the importance of assessing the family history when confronted with a child with suspected atopic disease or RRTI.

The association between respiratory infections and atopic diseases has been studied extensively. It has been suggested that children with atopic diseases may be more susceptible to respiratory infections due to airway inflammation and impaired innate immune function. ${ }^{27,28}$ On the contrary, other studies have proposed that infections may stimulate a patient's protective immunity and hereby reduce the risk of atopic diseases. ${ }^{29}$ In our cohort, children with a history of RRTI had a higher chance of asthma and allergic rhinitis, but a lower chance of atopic dermatitis and food allergy. Although a causal relationship cannot be demonstrated in this cross-sectional study, these data suggest that the association between RRTI and atopic diseases may be different per individual atopic disease entity.

There are a number of limitations to our study. First, this was a questionnaire-based survey. Thus, we measured the self-reported prevalence of atopic diseases and RRTI without objectively assessing clinical parameters such as lung capacity by spirometry. However, we did use validated and widely used instruments including the ISAAC questionnaires. ${ }^{14} \mathrm{~A}$ second limitation to our study is the cross-sectional study design. As we measured the prevalence rather than the incidence of diseases, it remains unknown whether the identified factors are a risk factor involved in the aetiology of the diseases. This study is strengthened by standardized data collection in a large group of children from both a birth cohort and hospital setting.

To conclude, our findings indicate that the demographic, environmental and family history risk factors for atopic diseases are distinct from the risk factors for RRTI. Thus, the changing prevalence of both disease entities might not be related to shared risk factors. 


\section{Acknowledgments}

The authors would like to thank the members of the Expert Network, Monique Gorissen, Bart van Ewijk, Walter Balemans, Maartje van Velzen, Frank Eskes, Gordon Slabbers, René van Gent, and participant of the Electronic Portal, M. Stadermann, for their collaboration and work within their centres. 


\section{References}

1. Asher M, Montefort S, Bjorksten B, et al. Worldwide time trends in the prevalence of symptoms of asthma, allergic rhinoconjunctivitis, and eczema in childhood. Lancet. 2006;368(9537):733-743.

2. Nwaru BI, Hickstein L, Panesar SS, et al. The epidemiology of food allergy in Europe: A systematic review and meta-analysis. Allergy. 2014;69(1):62-75.

3. Platts-Mills TAE. The allergy epidemics: 1870-2010. J Allergy Clin Immunol. 2015;136(1):3-13.

4. Troeger C, Blacker B, Khalil IA, et al. Estimates of the global, regional, and national morbidity, mortality, and aetiologies of lower respiratory infections in 195 countries, 1990-2016: a systematic analysis for the Global Burden of Disease Study 2016. Lancet Infect Dis. 2018;18(11):1191-1210.

5. Bach J. The effect of infections on susceptibility to autoimmune and allergic diseases. $N$ Engl J Med. 2002;347(12):911-920.

6. Yazdanbakhsh M, Kremsner PG, Van Ree R. Allergy, parasites and the hygiene hypothesis. Science. 2002;296(5567):490-494.

7. Mandhane PJ, Sears MR, Poulton R, et al. Cats and dogs and the risk of atopy in childhood and adulthood. J Allergy Clin Immunol. 2009;124(4):745-750.e4.

8. Benn CS, Melbye M, Wohlfahrt J, Björkstén B, Aaby P. Cohort study of sibling effect, infectious diseases, and risk of atopic dermatitis during first 18 months of life. $\mathrm{Br}$ Med $\mathrm{J}$. 2004;328(7450):1223-1226.

9. Hagerhed-Engman L, Bornehag CG, Sundell J, Åberg N. Day-care attendance and increased risk for respiratory and allergic symptoms in preschool age. Allergy Eur J Allergy Clin Immunol. 2006;61(4):447-453.

10. Toivonen L, Karppinen S, Schuez-Havupalo L, et al. Burden of recurrent respiratory tract infections in children: A prospective cohort study. Pediatr Infect Dis J. 2016;35(12):e362-e369.

11. Gallant MJ, Ellis AK. What can we learn about predictors of atopy from birth cohorts and cord blood biomarkers? Ann Allergy, Asthma Immunol. 2018;120(2):138-144.

12. Zomer-Kooijker K, van Erp FC, Balemans WAF, van Ewijk BE, van der Ent CK. The expert network and electronic portal for children with respiratory and allergic symptoms: Rationale and design. BMC Pediatr. 2013;13(1):9.

13. Katier N, Uiterwaal CSPM, De Jong BM, et al. The Wheezing Illnesses Study Leidsche Rijn (WHISTLER): Rationale and design. Eur J Epidemiol. 2004;19(9):895-903.

14. Asher MI, Keil U, Anderson HR, et al. International study of asthma and allergies in childhood (ISAAC): Rationale and methods. Eur Respir J. 1995;8(3):483-491.

15. Grüber $C$, Keil T, Kulig M, et al. History of respiratory infections in the first $12 \mathrm{yr}$ among children from a birth cohort. Pediatr Allergy Immunol. 2008;19(6):505-512.

16. Hill DA, Spergel JM. The Atopic March: Critical Evidence and Clinical Relevance. Ann Allergy Asthma Immunol. 2018;120(2):131-137.

17. Pali-Schöll I, Jensen-Jarolim E. Gender aspects in food allergy. Curr Opin Allergy Clin Immunol. 2019;19(3):249-255.

18. Chen W, Mempel M, Schober W, Behrendt H, Ring J. Gender difference, sex hormones, and immediate type hypersensitivity reactions. Allergy Eur J Allergy Clin Immunol. 2008;63(11):14181427.

19. Falagas ME, Mourtzoukou EG, Vardakas KZ. Sex differences in the incidence and severity of respiratory tract infections. Respir Med. 2007;101(9):1845-1863.

20. Siltanen $M$, Wehkalampi $K$, Hovi $P$, et al. Preterm birth reduces the incidence of atopy in adulthood. J Allergy Clin Immunol. 2011;127(4):935-942.

21. Du Toit $\mathrm{G}$, Roberts $\mathrm{G}$, Sayre $\mathrm{PH}$, et al. Randomized trial of peanut consumption in infants at risk for peanut allergy. N Engl J Med. 2015;372(9):803-813.

22. Qi C, Jiang Y, Yang I V., et al. Nasal DNA methylation profiling of asthma and rhinitis. J Allergy Clin Immunol. 2020:1-9. 


\section{Chapter 5}

23. Svanes C, Zock JP, Antó J, et al. Do asthma and allergy influence subsequent pet keeping? An analysis of childhood and adulthood. J Allergy Clin Immunol. 2006;118(3):691-698.

24. de Benedictis FM, Bush A. Recurrent lower respiratory tract infections in children. BMJ. 2018;362:k2698.

25. Picard C, Bobby Gaspar H, Al-Herz W, et al. International Union of Immunological Societies: 2017 Primary Immunodeficiency Diseases Committee Report on Inborn Errors of Immunity. J Clin Immunol. 2018;38(1):96-128.

26. Patarčić $\mathrm{I}$, Gelemanović $\mathrm{A}$, Kirin $\mathrm{M}$, et al. The role of host genetic factors in respiratory tract infectious diseases: Systematic review, meta-analyses and field synopsis. Sci Rep. 2015;5:1-10.

26. Patarčić I, Gelemanović A, Kirin $M$, et al. The role of host genetic factors in respiratory tract infectious diseases: Systematic review, meta-analyses and field synopsis. Sci Rep. 2015;5:1-10.

27. Busse WW, Lemanske Jr. RF, Gern JE. The Role of Viral Respiratory Infections in Asthma and Asthma Exacerbations. Lancet. 2010;376(9743):826-834.

28. Maurer M. Does allergy impair innate immunity? Leads and lessons from atopic dermatitis. Allergy. 2010;65:1351-1356.

29. Cruz AA, Cooper PJ, Figueiredo CA. Global issues in allergy and immunology: Parasitic infections and allergy. J Allergy Clin Immunol. 2018;140(5):1217-1228. 


\section{Supplemental tables}

Supplemental Table 1. Definition of asthma, allergic rhinitis, atopic dermatitis, food allergy and recurrent respiratory tract infections

\begin{tabular}{|c|c|c|}
\hline Outcome & Age category & Definition based on questions in the Electronic Portal \\
\hline Asthma & $1-18$ years & $\begin{array}{l}\text { Both questions have been answered with 'yes': } \\
\text { 1. Have you/has your child had wheezing or whistling in the chest } \\
\text { at any time in the last } 12 \text { months? } \\
\text { 2. Have you/has your child ever had asthma? }\end{array}$ \\
\hline $\begin{array}{l}\text { Allergic } \\
\text { rhinitis }\end{array}$ & $1-18$ years & $\begin{array}{l}\text { Both questions have been answered with 'yes': } \\
\text { 1. Have you/has your child ever had symptoms of sneezing, } \\
\text { running or blocked nose while not having a cold or the flu? } \\
\text { 2. Has these symptoms in the last } 12 \text { months been accompanied } \\
\text { by itchy-watery eyes? }\end{array}$ \\
\hline $\begin{array}{l}\text { Atopic } \\
\text { dermatitis }\end{array}$ & $1-18$ years & $\begin{array}{l}\text { Both questions have been answered with 'yes': } \\
\text { 1. Have you/has your child ever had an itchy rash which was } \\
\text { coming and going for at least } 6 \text { months? } \\
\text { 2. Has this rash been present during the last } 12 \text { months? }\end{array}$ \\
\hline Food allergy & $1-18$ years & $\begin{array}{l}\text { All three criteria have been fulfilled: } \\
\text { 1. Have you/has your child food allergy or do you think you } \\
\text { have/your child has food allergy? } \\
\text { 2. Have you/has your child developed symptoms within } 2 \text { hours } \\
\text { after eating the food? } \\
\text { 3. Did you/your child have one or more of the following } \\
\text { symptoms? } \\
\text { - Hives } \\
\text { - } \quad \text { Oral allergy (tingling in mouth or throat) } \\
\text { - Angio-oedema (swelling of mouth/tongue/lips and/or } \\
\text { - } \quad \text { Lowes) } \\
\text { - } \quad \text { squeaky respiration and/or shortness of breath) } \\
\quad \text { Upper respiratory symptoms (swelling of throat, } \\
\quad \text { soreness, coughing, running nose, blocked nose, } \\
\text { - } \quad \text { Voming, itchy/red/tearing eyes, feeling of swollen } \\
\text { - } \quad \text { Drowsiness } \\
\text { - Anaphylaxis (loss of consciousness) }\end{array}$ \\
\hline $\begin{array}{l}\text { Recurrent } \\
\text { respiratory }\end{array}$ & $1-2$ years & $\begin{array}{l}\geq 11 \text { upper respiratory infections per year and/or } \geq 2 \text { lower } \\
\text { respiratory infections in the past year }\end{array}$ \\
\hline $\begin{array}{l}\text { tract } \\
\text { infections }\end{array}$ & $2-5$ years & $\begin{array}{l}\geq 8 \text { upper respiratory infections per year and/or } \geq 2 \text { lower } \\
\text { respiratory infections in the past year }\end{array}$ \\
\hline (RRTI) & $\begin{array}{l}5-10 \text { years } \\
>10 \text { years }\end{array}$ & $\begin{array}{l}\geq 6 \text { upper respiratory infections per year and/or } \geq 2 \text { lower } \\
\text { respiratory infections in the past year } \\
\geq 4 \text { upper respiratory infections per year and/or } \geq 2 \text { lower } \\
\text { respiratory infections in the past year }\end{array}$ \\
\hline
\end{tabular}


Supplemental Table 2. Crude associations between risk factors and respectively any atopic disease and RRTI

\begin{tabular}{|c|c|c|c|c|}
\hline & \multicolumn{2}{|c|}{ Any atopic disease } & \multicolumn{2}{|l|}{ RRTI } \\
\hline & $\begin{array}{l}\text { Crude OR } \\
(95 \% \text { CI })\end{array}$ & $P$ value $^{+}$ & $\begin{array}{l}\text { Crude OR } \\
(95 \% \mathrm{CI})\end{array}$ & $P$ value $^{+}$ \\
\hline \multicolumn{5}{|l|}{ Demographics } \\
\hline \multicolumn{5}{|l|}{ Age (in years) } \\
\hline 5 to 8 (vs. 1 to 4$)$ & $1.15(1.00-1.32)$ & 0.05 & $0.87(0.75-1.01)$ & 0.07 \\
\hline 9 to 12 (vs. 1 to 4 ) & $1.47(1.26-1.72)$ & $<0.001$ & $0.78(0.66-0.91)$ & $<0.01$ \\
\hline 13 to 17 (vs. 1 to 4 ) & $2.89(2.34-3.58)$ & $<0.001$ & $1.15(0.94-1.41)$ & 0.17 \\
\hline Female sex & $0.71(0.63-0.80)$ & $<0.001$ & $0.92(0.82-1.03)$ & 0.15 \\
\hline \multicolumn{5}{|l|}{ Gestational age } \\
\hline $37-43$ weeks $($ vs. < 37) & $0.99(0.82-1.18)$ & 0.88 & $0.71(0.59-0.85)$ & $<0.001$ \\
\hline$\geq 43$ weeks (vs. < 37) & $1.12(0.71-1.77)$ & 0.63 & $0.67(0.42-1.06)$ & 0.08 \\
\hline Low birth weight (vs. normal) & $0.86(0.70-1.06)$ & 0.15 & $1.24(1.01-1.53)$ & 0.04 \\
\hline Non-Dutch mother (vs. Dutch) & $1.08(0.85-1.36)$ & 0.55 & $0.86(0.66-1.13)$ & 0.28 \\
\hline Non-Dutch father (vs. Dutch) & $1.32(1.08-1.62)$ & $<0.01$ & $0.96(0.78-1.18)$ & 0.72 \\
\hline \multicolumn{5}{|l|}{ Education level mother } \\
\hline Middle (vs. low) & $1.28(1.03-1.59)$ & 0.02 & $0.79(0.64-0.98)$ & 0.03 \\
\hline High (vs. low) & $0.99(0.80-1.22)$ & 0.93 & $0.56(0.46-0.69)$ & $<0.001$ \\
\hline \multicolumn{5}{|l|}{ Education level father } \\
\hline Middle (vs. low) & $1.00(0.82-1.22)$ & 0.98 & $0.70(0.58-0.85)$ & $<0.001$ \\
\hline High (vs. low) & $0.89(0.73-1.07)$ & 0.21 & $0.56(0.46-0.68)$ & $<0.001$ \\
\hline \multicolumn{5}{|l|}{ Environment } \\
\hline \multicolumn{5}{|l|}{ Exclusive breastfeeding } \\
\hline$<6$ months (vs. never) & $1.02(0.87-1.19)$ & 0.83 & $0.79(0.68-0.93)$ & $<0.01$ \\
\hline$\geq 6$ months (vs. never) & $1.08(0.92-1.26)$ & 0.35 & $0.83(0.71-0.96)$ & 0.02 \\
\hline Pacifier use & $1.08(0.96-1.22)$ & 0.18 & $1.14(1.01-1.29)$ & 0.03 \\
\hline Having siblings & $0.93(0.79-1.08)$ & 0.32 & $1.05(0.85-1.29)$ & 0.65 \\
\hline \multicolumn{5}{|l|}{ Number of older siblings } \\
\hline 1 (vs. 0 ) & $0.86(0.76-0.98)$ & 0.02 & $1.05(0.92-1.21)$ & 0.47 \\
\hline 2 (vs. 0 ) & $0.79(0.64-0.96)$ & 0.02 & $1.18(0.96-1.45)$ & 0.12 \\
\hline$\geq 3($ vs. 0$)$ & $0.86(0.54-1.35)$ & 0.50 & $1.01(0.56-1.82)$ & 0.97 \\
\hline Day care attendance & $1.03(0.88-1.20)$ & 0.75 & $1.02(0.87-1.19)$ & 0.83 \\
\hline Urban living environment (vs. rural) & $0.75(0.67-0.84)$ & $<0.001$ & $0.73(0.65-0.82)$ & $<0.001$ \\
\hline Having a pet dog & $0.98(0.84-1.15)$ & 0.81 & $1.50(1.28-1.77)$ & $<0.001$ \\
\hline Having a pet cat & $0.75(0.65-0.87)$ & $<0.001$ & $1.02(0.87-1.19)$ & 0.83 \\
\hline Maternal smoking during pregnancy & $0.92(0.68-1.24)$ & 0.58 & $1.68(1.22-2.31)$ & $<0.01$ \\
\hline Indoor smoking & $1.26(0.95-1.67)$ & 0.10 & $1.68(1.28-2.20)$ & $<0.001$ \\
\hline Adopted & $1.14(0.67-1.91)$ & 0.63 & $0.50(0.28-0.90)$ & 0.02 \\
\hline Vaccinated & $0.79(0.65-0.96)$ & 0.02 & $0.96(0.79-1.16)$ & 0.64 \\
\hline Carpet floor house (vs. solid) & $2.06(1.24-3.43)$ & $<0.01$ & $1.01(0.65-1.58)$ & 0.95 \\
\hline Carpet floor bedroom child (vs. solid) & $0.79(0.68-0.92)$ & $<0.01$ & $0.85(0.73-1.00)$ & 0.06 \\
\hline \multicolumn{5}{|l|}{ Family history } \\
\hline Asthma & $1.67(1.47-1.88)$ & $<0.001$ & $1.29(1.15-1.45)$ & $<0.001$ \\
\hline Allergic rhinitis & $1.70(1.51-1.91)$ & $<0.001$ & $0.99(0.87-1.13)$ & 0.92 \\
\hline Atopic dermatitis & $1.94(1.72-2.19)$ & $<0.001$ & $1.10(0.98-1.23)$ & 0.12 \\
\hline Food allergy & $1.74(1.51-2.00)$ & $<0.001$ & $1.09(0.95-1.24)$ & 0.22 \\
\hline RRTI & $1.15(1.02-1.30)$ & 0.02 & $1.98(1.75-2.24)$ & $<0.001$ \\
\hline
\end{tabular}


Supplemental Table 2. Crude associations between risk factors and respectively any atopic disease and RRTI (continued)

\begin{tabular}{|c|c|c|c|c|}
\hline & \multicolumn{2}{|c|}{ Any atopic disease } & \multicolumn{2}{|l|}{ RRTI } \\
\hline & $\begin{array}{l}\text { Crude OR } \\
(95 \% \mathrm{CI})\end{array}$ & $P$ value ${ }^{+}$ & $\begin{array}{l}\text { Crude OR } \\
(95 \% \mathrm{CI})\end{array}$ & $P$ value ${ }^{\dagger}$ \\
\hline \multicolumn{5}{|l|}{ Comorbidities } \\
\hline Asthma & NA & & $2.30(1.97-2.68)$ & $<0.001$ \\
\hline Allergic rhinitis & NA & & $1.55(1.36-1.76)$ & $<0.001$ \\
\hline Atopic dermatitis & NA & & $0.91(0.81-1.03)$ & 0.14 \\
\hline Food allergy & NA & & $0.91(0.74-1.11)$ & 0.36 \\
\hline RRTI & $1.29(1.13-1.46)$ & $<0.001$ & NA & \\
\hline
\end{tabular}

${ }^{+}$Significant $P$ values are in bold.

Abbreviations: $\mathrm{Cl}$, confidence interval; OR, odds ratio. 


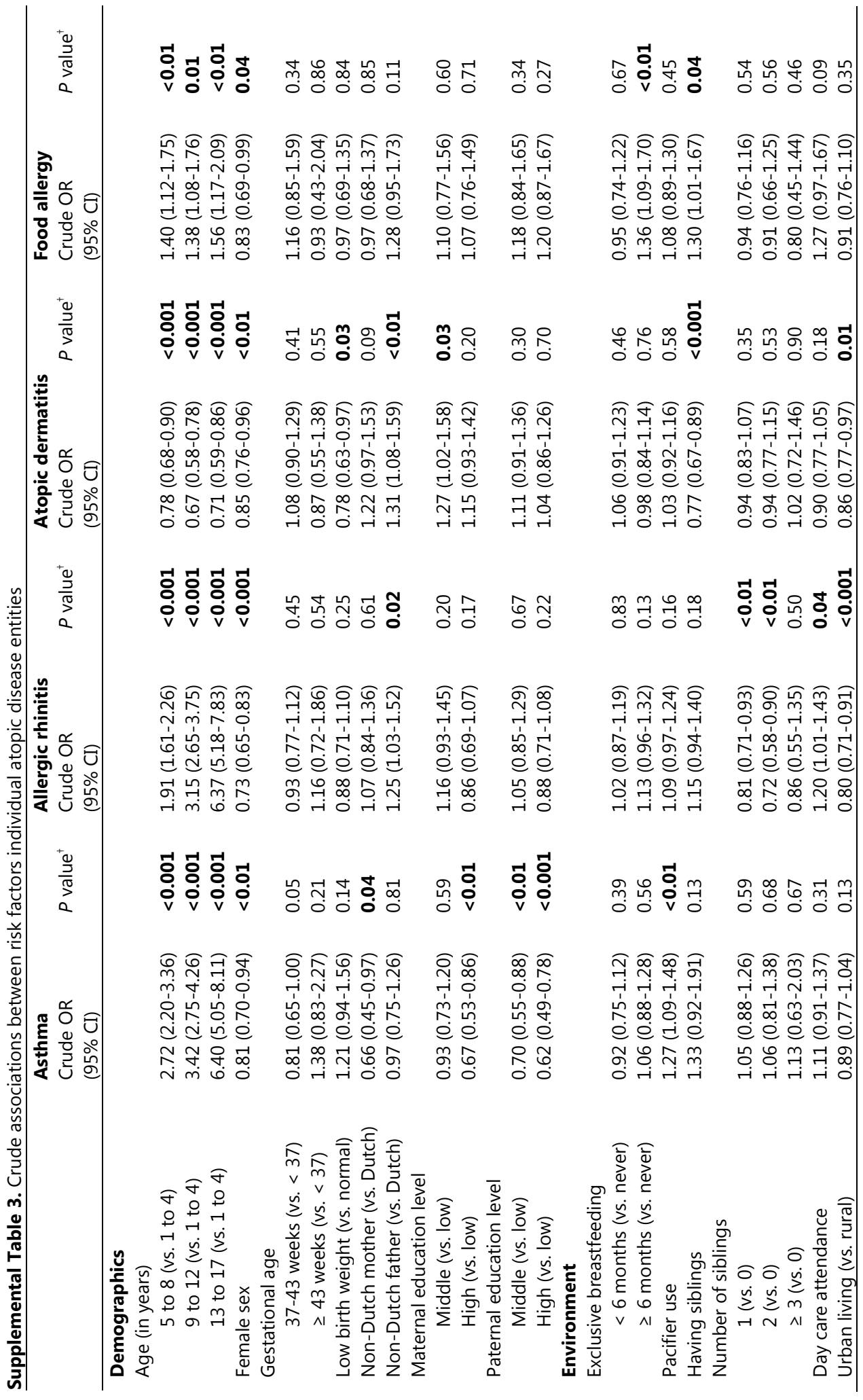




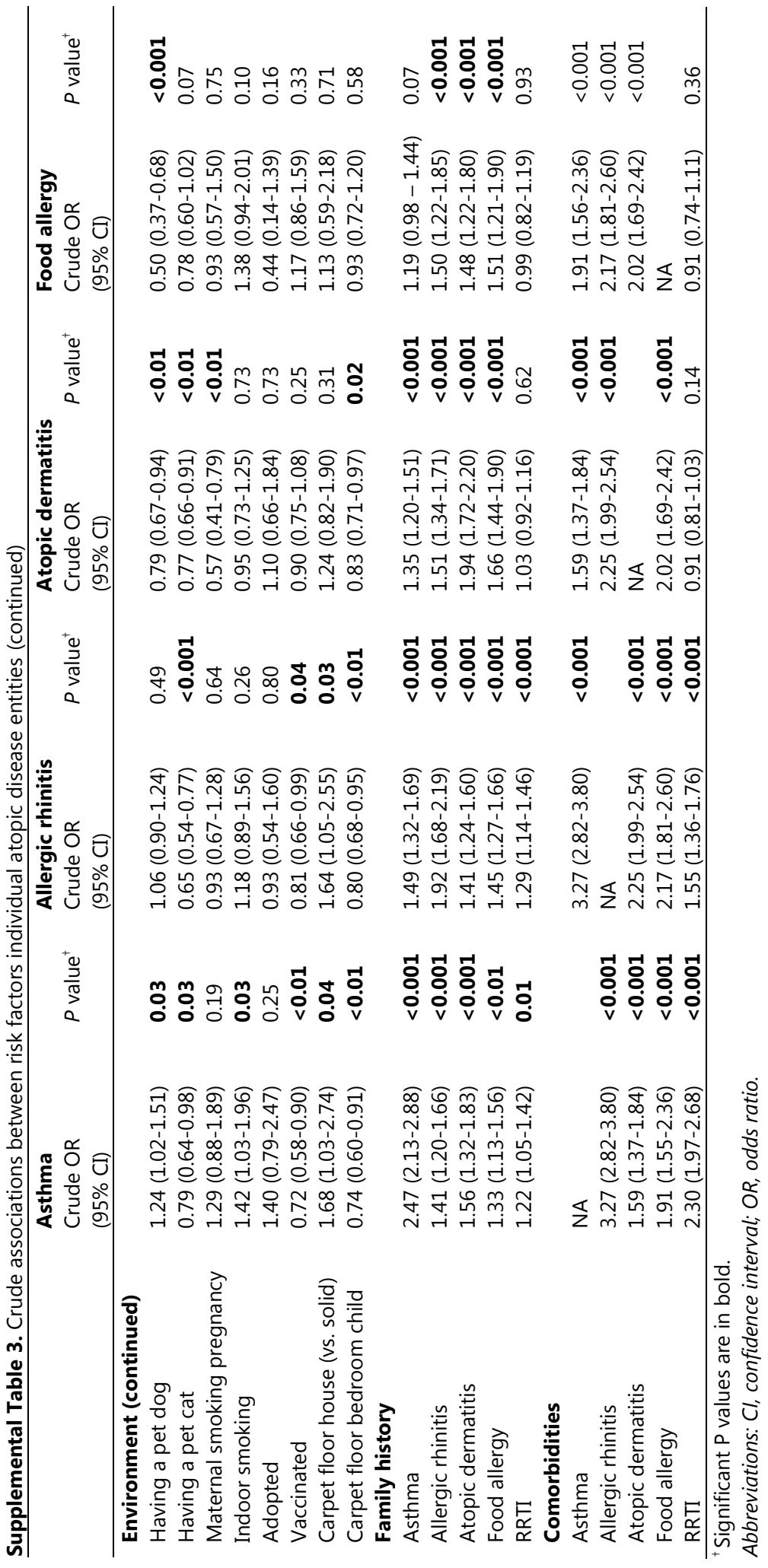




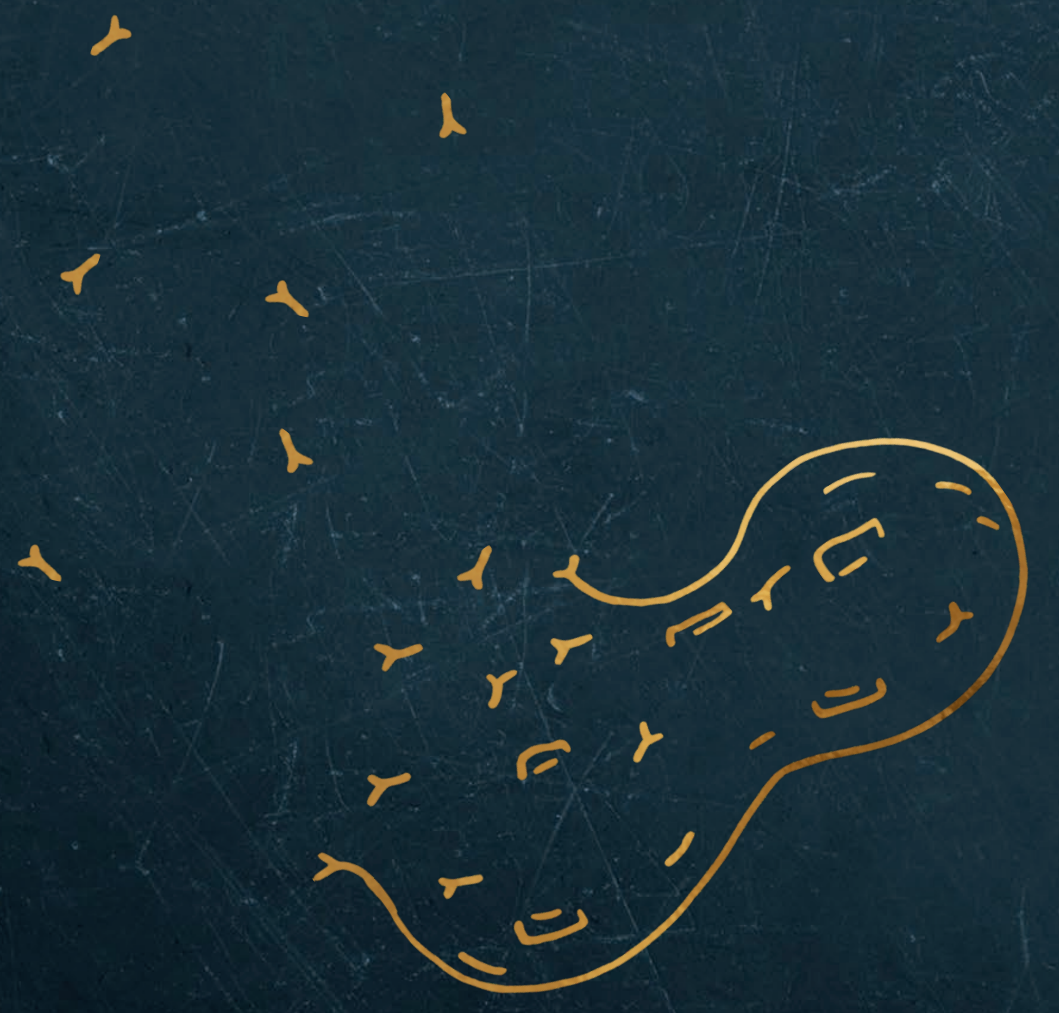




\section{Chapter 6}

\section{The impact of oral food challenges for food allergy on quality of life: a systematic review}




\section{Abstract}

\section{Background}

Food allergy significantly impairs health-related quality of life (HRQL). Currently, it is still unknown whether diagnostic interventions for food allergy improve HRQL. We aim to assess the impact of diagnostic interventions for food allergy on HRQL.

\section{Methods}

A systematic search was performed in MEDLINE, Embase, Cochrane Library and CINAHL focused on patients with a (suspected) food allergy who underwent diagnostic interventions (i.e. skin prick test, specific IgE or oral food challenges $(\mathrm{OFC})$ ), and in whom HRQL was assessed. The mean difference between HRQL before and after the diagnostic intervention was calculated. A minimal clinically important difference of 0.5 was considered clinically relevant for the Food Allergy Quality of Life Questionnaire.

\section{Results}

Seven of 1465 original identified publications were included in which the impact of an OFC on HRQL was investigated (total patients $n=1370$ ). No other diagnostic interventions were investigated. Food allergy specific parent-reported HRQL improved significantly after an OFC irrespective of the outcome in children with a suspected food allergy in two publications. The change was considered clinically relevant in one of two publications. In addition, parent-reported HRQL improved after an OFC to assess the eliciting dose in children with a confirmed food allergy. The parental burden was significantly reduced after an OFC to assess resolution of food allergy. A meta-analysis could not be performed due to the limited numbers of, and considerable heterogeneity between, eligible publications.

\section{Conclusion}

An OFC is associated with an improved food allergy specific HRQL and a reduced parental burden of food allergy. 


\section{Introduction}

The prevalence of food allergy is thought to be increasing during recent decades. ${ }^{1}$ Previous studies have shown that up to $35 \%$ of the population reports adverse reactions to food, while between $1 \%$ to $3 \%$ has a food allergy confirmed by an oral food challenge (OFC). ${ }^{2,3}$

Currently no curative treatment for food allergy is available. Patients are advised to follow an elimination diet and to carry emergency medication to avoid or treat possible life-threatening allergic reactions. ${ }^{4}$ Hence, patients with a food allergy are faced with dietary and social restrictions. Due to these restrictions, and fear of an allergic reaction, patients with a food allergy have a significantly impaired food allergy specific health-related quality of life (HRQL) ${ }^{5,6}$ In addition, patients with a food allergy reported poorer generic HRQL than the general population and patients with diabetes mellitus type 1 but better generic HRQL than patients with rheumatoid arthritis, asthma and irritable bowel syndrome. ${ }^{7}$ This may be explained by the fact that patients with a food allergy live with constant vigilance and fear of an allergic reaction although they do not have daily chronic symptoms like patients with rheumatoid arthritis, asthma and irritable bowel syndrome. ${ }^{8}$

An accurate diagnosis of food allergy is highly important to minimize unnecessary elimination diets in non-allergic patients on the one hand and avoid allergic reactions in patients with a food allergy on the other hand. Currently the diagnostic process for food allergy consists of a careful clinical and dietary history and sensitization tests including the level of specific IgE (sIgE) to the suspected food and/or a skin prick test (SPT). ${ }^{9}$ However, these sensitization tests have a relatively low specificity depending on the allergen. ${ }^{10}$ The current reference standard to assess a food allergy, the threshold and the severity of the clinical reaction is an oral food challenge (OFC). In short, increasing amounts of food allergen or placebo are given with close monitoring in a hospital setting with emergency equipment. ${ }^{11,12}$

The increasing prevalence of food allergy and the significant impact of food allergy on HRQL of affected patients give cause for careful consideration of current diagnostic strategies. A comprehensive assessment on the impact of diagnostic interventions for food allergy on HRQL of patients is important as these diagnostic interventions might improve HRQL. Therefore, the aim of this review is to provide a systematic synthesis of the current evidence on the impact of diagnostic interventions for food allergy on HRQL. 


\section{Methods}

\section{Search strategy}

This systematic review was conducted according to a previously developed protocol registered on the international prospective register of systematic reviews (PROSPERO) and reported according to the PRISMA checklist. ${ }^{13,14}$

We developed an extensive search strategy to identify all publications relevant to our research question from electronic bibliographic databases using keywords and Medical Subject Headings. The search combined keywords and synonyms for the domain (patients with a suspected or a confirmed food allergy), the determinant (diagnostic interventions for food allergy: specific IgE (sIgE), skin prick testing (SPT), oral food challenges (OFC), or component resolved diagnostics), and the outcome (food allergy specific or generic HRQL outcome measures). The search strategy was initially developed for the MEDLINE database and then adapted for use on other databases. The full search strategy is published in the supplementary material (Supplemental 1). Four databases were searched from inception until July 6th 2017: MEDLINE, EMBASE, the Cochrane Library and CINAHL. All identified citations were imported into Rayyan for de-duplication and title and abstract screening. ${ }^{15}$ All identified publications were screened by two authors (HK, FE) independently. Subsequently, all potentially relevant articles were screened full text by the same two authors independently and assessed for eligibility. The references and citations of all the publications that were screened full text were reviewed to identify any additional relevant sources. The citations were analyzed using Scopus. ${ }^{16}$ The reasons for exclusion of the publications that were screened full text are listed in the supplemental material (Supplemental Table 1). Any discrepancies between two authors were resolved by discussion and consensus, or by consulting a third reviewer $(\mathrm{TL})$ if necessary.

\section{Eligibility criteria}

We included publications in English, Dutch, German, French or Spanish and did not restrict on publication year. We excluded publications if no original outcome data were reported, such as other systematic reviews, meta-analyses or editorials. We included publications of children or adults with a suspected or a confirmed food allergy if a diagnostic intervention for a food allergy was performed and HRQL scores were measured or could be calculated. In patients with a suspected food allergy the aim of the diagnostic intervention was to confirm or exclude the diagnosis of a food allergy while the aim of diagnostic interventions in patients with a confirmed food allergy was either to assess the threshold, the severity or the resolution of a food allergy. Publications were only included if a validated food allergy specific or generic HRQL instrument was used to measure a change before 
and after a diagnostic intervention, or a difference in patients with or without a diagnostic intervention. We contacted the study authors of abstract publications and original publications with incomplete HRQL outcome data to obtain additional information on the study methods and the original data. When we were not able to acquire further details on abstract publications, these publications were excluded. A complete overview of inclusion and exclusion criteria is displayed in the supplemental material (Supplemental Table 2).

\section{Quality of paper assessment}

The included publications were assessed for risk of bias in duplicate (HK, FE) according to a modified version of the Quality in Prognostic Studies (QUIPS) tool (Supplemental Table 3). ${ }^{17}$ The QUIPS tool considers six domains of potential bias and every domain comprises several prompting items to consider. All items were scored (yes, partly, no or unsure) by two authors independently. Subsequently, the six domains of the individual publications were graded for the risk of bias and last, each publication as well as the six domains of all publications was graded for the overall risk of bias (high, moderate or low). Any discrepancies between two authors were again resolved by discussion and consensus, or by consulting a third reviewer $(\mathrm{TL})$ if necessary.

\section{Outcome assessment}

HRQL can be measured using disease-specific or generic HRQL questionnaires. Disease-specific HRQL questionnaires are able to measure food allergy related impairments, such as the dietary and social restrictions, and the fear of allergic reactions. In contrast to disease-specific HRQL questionnaires, generic HRQL questionnaires facilitate direct comparison to other populations. We included publications that evaluated disease-specific or generic HRQL before and after a diagnostic intervention, and publications that evaluated the difference in diseasespecific or generic HRQL in patients with or without a diagnostic intervention.

Disease-specific questionnaires used to evaluate HRQL in food allergic patients in the eligible publications included the self-administered Food Allergy Quality of Life Questionnaire (FAQLQ) with age-specific adaptations: the Child Form (FAQLQ-CF) for children 8 to 12 years of age, the Teenager Form (FAQLQ-TF) for adolescents 13-17 years of age, and the Adult Form (FAQLQ-AF) for adults $\geq 18$ years of age. In addition, the Parent Form (FAQLQ-PF) was available to measure parent-reported HRQL of children 0-12 years of age. The FAQLQ-CF contains 24 items and 4 domains (Allergen Avoidance, Risk of Accidental Exposure, Emotional Impact, Dietary restrictions), the FAQLQ-TF contains 23 items and 3 domains (Allergen Avoidance, Risk of Accidental Exposure, Emotional Impact), the FAQLQ-AF contains 29 items and 4 domains (Allergen Avoidance, Risk of Accidental Exposure, Emotional Impact, Food Allergy-related Health), and the FAQLQ-PF contains 30 
items and 3 domains (Emotional Impact, Food Anxiety, Social Dietary Limitations). The FAQLQ-items are scored on a seven-point scale. The longitudinal validity and responsiveness of the FAQLQ has been demonstrated. ${ }^{18,19}$ In addition, the Food Allergy Quality of Life Parental Burden Questionnaire (FAQL-PB) was used to assess the effect of a child with food allergy on caregiver HRQL. The FAQL-PB is a validated questionnaire which contains 17 items. $^{20}$

Generic questionnaires used to evaluate HRQL in the eligible publications were the Paediatric Quality of Life Inventory 4.0 (PedsQL ${ }^{\mathrm{TM}}$ 4.0) in children and the World Health Organization generic Quality of Life scale (WHOQOL-BREF) in parents. The PedsQL ${ }^{\mathrm{TM}} 4.0$ is a validated generic HRQL questionnaire for children 8 to 12 years of age which contains 23 items and 4 domains (Physical, Emotional, Social, School). The WHOQOL-BREF is a validated generic HRQL questionnaire for adults which contains 26 items and 4 domains (Physical, Psychological, Social, Environmental).

\section{Data analysis}

Full details on the publications, patients, diagnostic intervention(s), and outcome (HRQL questionnaire) were gathered. Publications in patients with a suspected food allergy were analyzed separately from publications in patients with a confirmed food allergy because HRQL is associated with perceived disease severity. ${ }^{21}$ Furthermore, the impact of an OFC on HRQL might be profoundly different in patients with a suspected or a confirmed food allergy because the aim of an OFC in patients with a suspected food allergy is to confirm or exclude the diagnosis of a food allergy while the aim of an OFC in patients with a confirmed food allergy is either to assess the threshold, the severity or the resolution of a food allergy.

For publications using the Food Allergy Quality of Life Questionnaires (FAQLQ) we calculated the mean difference (MD) before and after the diagnostic intervention with a $95 \%$ confidence interval $(95 \% \mathrm{CI})$. If the $95 \%$ CI of the MD was not available in the original publication and could not be provided by the study authors we computed this value using the SD of the difference scores. ${ }^{22}$ To compute the SD of the difference scores in paired data the correlation coefficient $(r)$ between prescores and post-scores is required. We assumed a correlation of $r=0.5$, and performed a sensitivity analysis to evaluate the impact of this assumption using a range of plausible correlation ( $r=0.2$ and $r=0.8)$. The MD score of the FAQLQ is meaningful as this change score can be interpreted using the minimal clinically important difference (MCID). The MCID is the smallest change score that is considered clinically relevant. ${ }^{23}$ The MCID for the FAQLQ is 0.5 as estimated previously using a distribution-based method. ${ }^{18}$

For publications using other HRQL questionnaires than the FAQLQ, or if the MD of the FAQLQ could not be calculated, we estimated the standardized mean difference (SMD) before and after the diagnostic intervention with a 95\% CI using Cohen's 
statistics for paired data. ${ }^{24}$ Again, the correlation coefficient $r$ was imputed to calculate the SD within groups if needed. Based on Cohen's criteria, a SMD of 0.2 is considered small, 0.5 is moderate and $>0.8$ is large. $^{24}$

The MD and SMD were calculated in such a way that their direction was positive. Thus, a positive MD or SMD indicated an improved HRQL. If three or more publications reported HRQL outcomes on the same questionnaire in comparable groups of patients with comparable diagnostic interventions, the results were pooled using the random effects model. ${ }^{25}$

For publications with a cross-sectional study design, a difference in mean HRQL scores between patients who underwent an OFC and those who did not undergo an OFC was evaluated using the two-sided independent t-test.

All data were extracted using standardized pre-piloted data extraction forms in Microsoft Excel 2010, and forest plots were created using GraphPad Prism 7.02 (GraphPad Software, Inc, San Diego, CA)

\section{Results}

\section{Selection of eligible publications}

The search results are summarized in a flowchart in Figure 1. We selected 31 of 1465 original identified publications for the full text eligibility screening. Seven eligible publications were included in the final systematic review. Reasons for exclusion after the full text screening were: no diagnostic intervention was investigated $(n=10)$, the publication did not report original data $(n=4)$, only nonallergic patients were included $(n=3)$, the health-related quality of life (HRQL) questionnaire was not validated $(n=3)$, no HRQL questionnaire was used $(n=2)$ or no change or difference in HRQL was assessed $(n=2)$. No new relevant sources were identified by checking references and analyzing citations. 


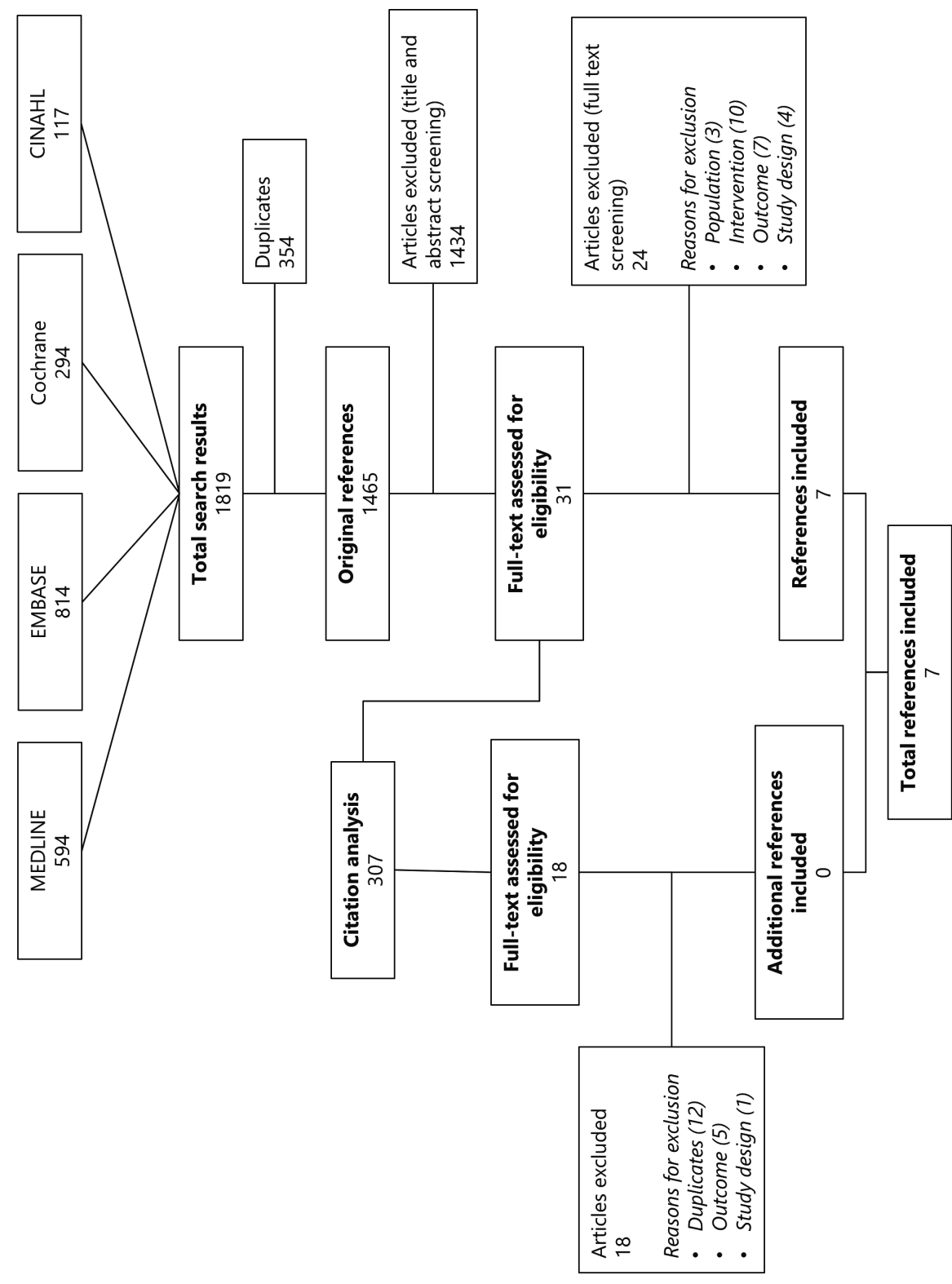

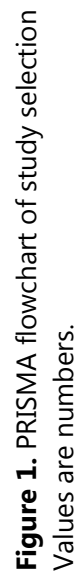




\section{Characteristics of included publications}

The characteristics of the seven included publications are summarized in Table 1. Overall, 1370 patients (ranging between 54 and 420 per study) were recruited between 2007 and 2016 in tertiary care. ${ }^{18,26-30}$ All included publications investigated the impact of an OFC on HRQL. No publications were identified in which the impact of other diagnostic interventions on HRQL was investigated.

Four of seven included publications assessed HRQL in patients with a suspected food allergy. ${ }^{18,19,27,29}$ Three of these four publications compared HRQL before and after an OFC. One of the four publications compared the HRQL between patients who underwent OFC and patients who were on the waiting list for OFC or who were considered food allergic by a physician. ${ }^{19}$

Two of seven included publications assessed HRQL before and after an OFC in patients with a confirmed food allergy. The aim of the OFC was to evaluate the eliciting dose ${ }^{26}$ or to assess resolution of food allergy. ${ }^{30}$ In one of these two publications HRQL was also assessed in patients that did not undergo an OFC. These patients were considered food allergic by a physician. ${ }^{30}$

Finally, one of seven included publications assessed HRQL in patients with a confirmed food allergy at a single point in time in both patients previously diagnosed through an OFC and in patients considered food allergic but who did not undergo an OFC. ${ }^{28}$

The risk of bias was considered high in three ${ }^{28-30}$, moderate in three ${ }^{18,19,26}$ and low in one publication ${ }^{27}$ (Table 2). The complete results of the risk of bias assessment are published in the supplemental material (Supplemental Table 4). 


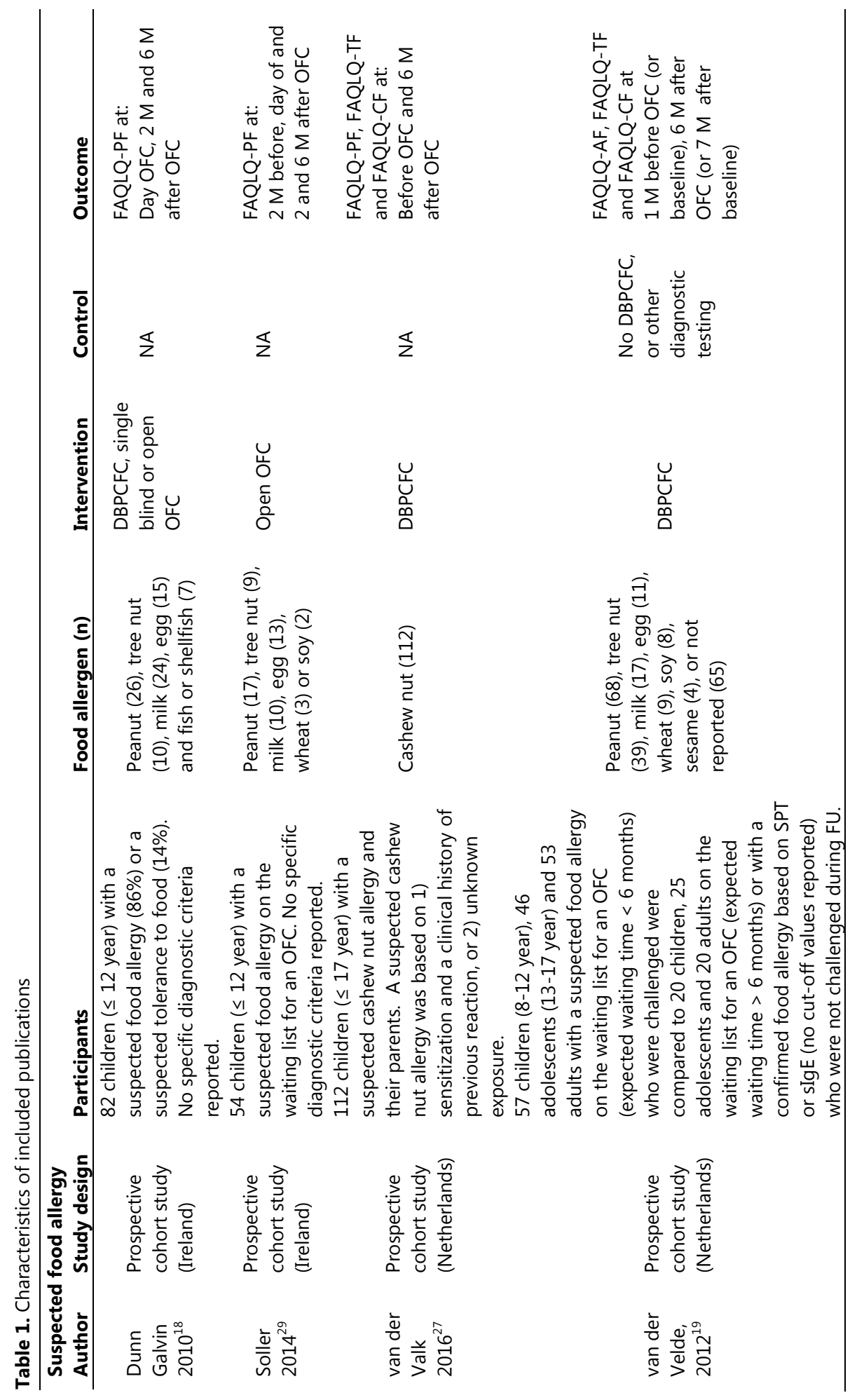




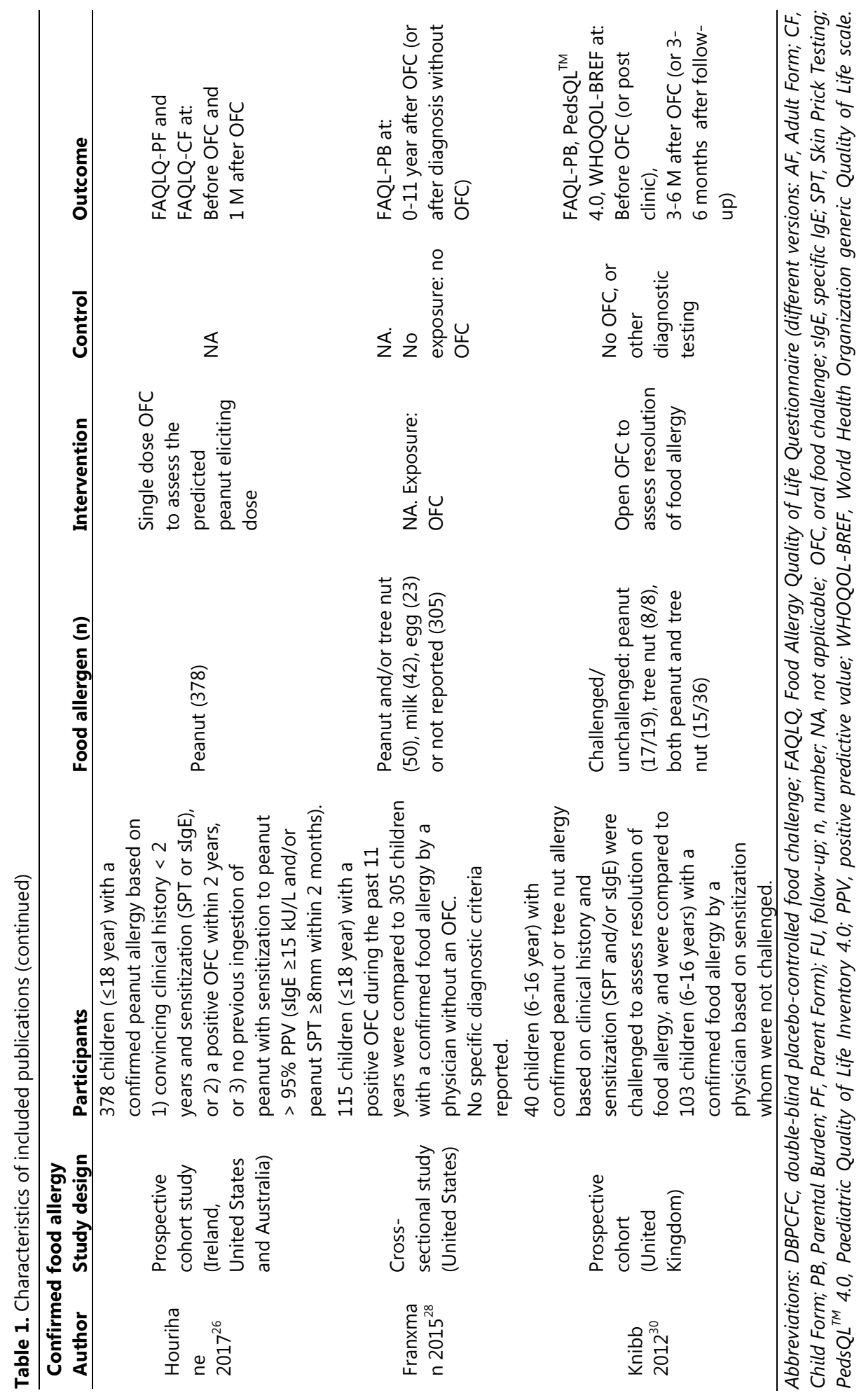


Table 2. Risk of bias assessment

\begin{tabular}{|c|c|c|c|c|c|c|c|c|}
\hline & Hourihane & $\begin{array}{l}\text { Van der } \\
\text { Valk }\end{array}$ & $\begin{array}{l}\text { Franx } \\
\text { man }\end{array}$ & Soller & Knibb & $\begin{array}{l}\text { Van der } \\
\text { Velde }\end{array}$ & $\begin{array}{l}\text { Dunn } \\
\text { Galvin }\end{array}$ & $\begin{array}{l}\text { Overall } \\
\text { risk of } \\
\text { bias }\end{array}$ \\
\hline & 2017 & 2016 & 2015 & 2014 & 2012 & 2012 & 2010 & \\
\hline $\begin{array}{l}\text { Study } \\
\text { participation }\end{array}$ & L & L & $\mathrm{H}$ & M & M & L & L & M \\
\hline $\begin{array}{l}\text { Study Attrition } \\
\text { Diagnostic }\end{array}$ & L & M & $\mathrm{H}$ & M & $\mathrm{H}$ & $\mathrm{H}$ & M & $\mathrm{H}$ \\
\hline $\begin{array}{l}\text { Intervention } \\
\text { Measurement }\end{array}$ & L & L & M & L & M & L & $\mathrm{H}$ & M \\
\hline $\begin{array}{l}\text { Outcome } \\
\text { Measurement }\end{array}$ & L & L & L & L & L & L & L & L \\
\hline $\begin{array}{l}\text { Study } \\
\text { Confounding } \\
\text { Statistical }\end{array}$ & M & L & L & $\mathrm{H}$ & $M$ & L & $M$ & $M$ \\
\hline $\begin{array}{l}\text { Analysis and } \\
\text { Reporting }\end{array}$ & $\mathrm{H}$ & L & L & M & L & L & L & M \\
\hline $\begin{array}{l}\text { Overall risk of } \\
\text { bias }\end{array}$ & $M$ & L & $\mathrm{H}$ & $\mathrm{H}$ & $\mathrm{H}$ & M & M & \\
\hline
\end{tabular}

Abbreviations: $H$, high; L, low; $M$, moderate.

\section{Quality of life in patients with a suspected food allergy}

In patients with a suspected food allergy who underwent an OFC with positive, negative and inconclusive outcomes combined, food allergy specific HRQL significantly improved after an OFC in four of eight groups in three publications: in both children and adults in one publication ${ }^{19}$, and in parents in two publications ${ }^{18,29}$ (Figure 2a). The improved HRQL was only clinically relevant in parents in one publication, with a $95 \% \mathrm{CI}$ of the mean difference (MD) exceeding the minimal clinically important difference (MCID). ${ }^{29}$

Subgroup analyses were also performed for the different OFC outcomes, i.e. positive, negative and inconclusive OFC. This showed that in patients with a positive OFC outcome (i.e. food allergic patients), parent-reported food allergy specific HRQL significantly improved after an OFC in two of three publications and this change was clinically relevant in one publication (Figure $2 b$ ). ${ }^{18,29}$ In patients with a negative OFC outcome (i.e. non-allergic patients), food allergy specific HRQL significantly improved after an OFC in six of eight groups in four publications: again in parents included in two publications ${ }^{18,29}$, but also in adults, adolescents and children in one publication ${ }^{19}$, and in adolescents in another publication (Figure 2c). ${ }^{27}$ The improved HRQL was only clinically relevant in one publication in which parent-reported HRQL was assessed. ${ }^{29}$ In patients with an inconclusive OFC outcome food allergy specific HRQL did not improve after an OFC (Figure 2d). ${ }^{19}$

In two publications HRQL after the OFC was followed up in time, and was measured at 2 and 6 months after the OFC. These two publications showed that after a negative OFC the parent-reported HRQL further improved between two and six 
months and this improvement was significant and clinically relevant (Supplemental Figure 1). ${ }^{18,29}$

In addition to the inclusion of patients that underwent an OFC, one publication also included patients that did not undergo an OFC. ${ }^{19}$ All patients who underwent an OFC were suspected of having a food allergy, while the patients who did not undergo an OFC were either suspected of having a food allergy and were on the waiting list for OFC or were already diagnosed with a food allergy by a physician (Table 1). No significant difference was observed between food allergy specific HRQL at baseline and after 7 months in children, and adolescents (Supplemental Figure 2a). Furthermore, no significant difference was observed between the MD in HRQL in patients that underwent an OFC compared to the patients that did not undergo an OFC (Supplemental Figure 3).

\section{Domain specific quality of life in patients with a suspected food allergy}

The domain-specific parent-reported HRQL values are shown in Figure 3, and domain-specific HRQL values in children and adolescents in Supplemental Figure 4. In one publication no domain-specific HRQL values were available. ${ }^{19}$

In parents of children with all OFC outcomes combined, parent-reported HRQL significantly improved after an OFC in all three domains in two of three publications but this change was clinically relevant only in the domains of 'food anxiety' and 'social and dietary impact' in one publication (Figure 3a). ${ }^{18,29}$ In children and adolescents with all OFC outcomes combined, food allergy specific HRQL improved in the domain of allergen avoidance in children and in the domain of emotional impact in adolescents in one publication, although not clinically relevant (Supplemental Figure 4a). ${ }^{27}$

In parents of children with a positive OFC outcome (i.e. food-allergic patients), parent-reported food allergy specific HRQL significantly improved significantly after an OFC in the domain of 'emotional impact' in two of three publications ${ }^{18,29}$ and this change was clinically relevant in one publication. ${ }^{18}$ In one of these two publications, food allergy specific HRQL significantly improved after an OFC in the domains of 'social and dietary impact' and 'food anxiety'. ${ }^{29}$ This change in HRQL was clinically relevant in the domain of 'social and dietary impact' only. In children and adolescents with a positive OFC outcome, food allergy specific HRQL did not improve after a positive OFC outcome (Supplemental Figure 4b).

In parents of children with a negative OFC outcome (i.e. non-allergic patients), parent-reported food allergy specific HRQL significantly improved after an OFC in all three domains in two of three publications ${ }^{18,29}$, and this change was clinically relevant in all domains in one publication ${ }^{29}$ and in the domain of 'social and dietary 
impact' in the other publication ${ }^{18}$ (Figure 3c). Furthermore, HRQL significantly improved in the domains of 'risk accidental exposure' and 'emotional impact' in adolescents included in one publication although not clinically relevant (Supplemental Figure 4c). ${ }^{27}$

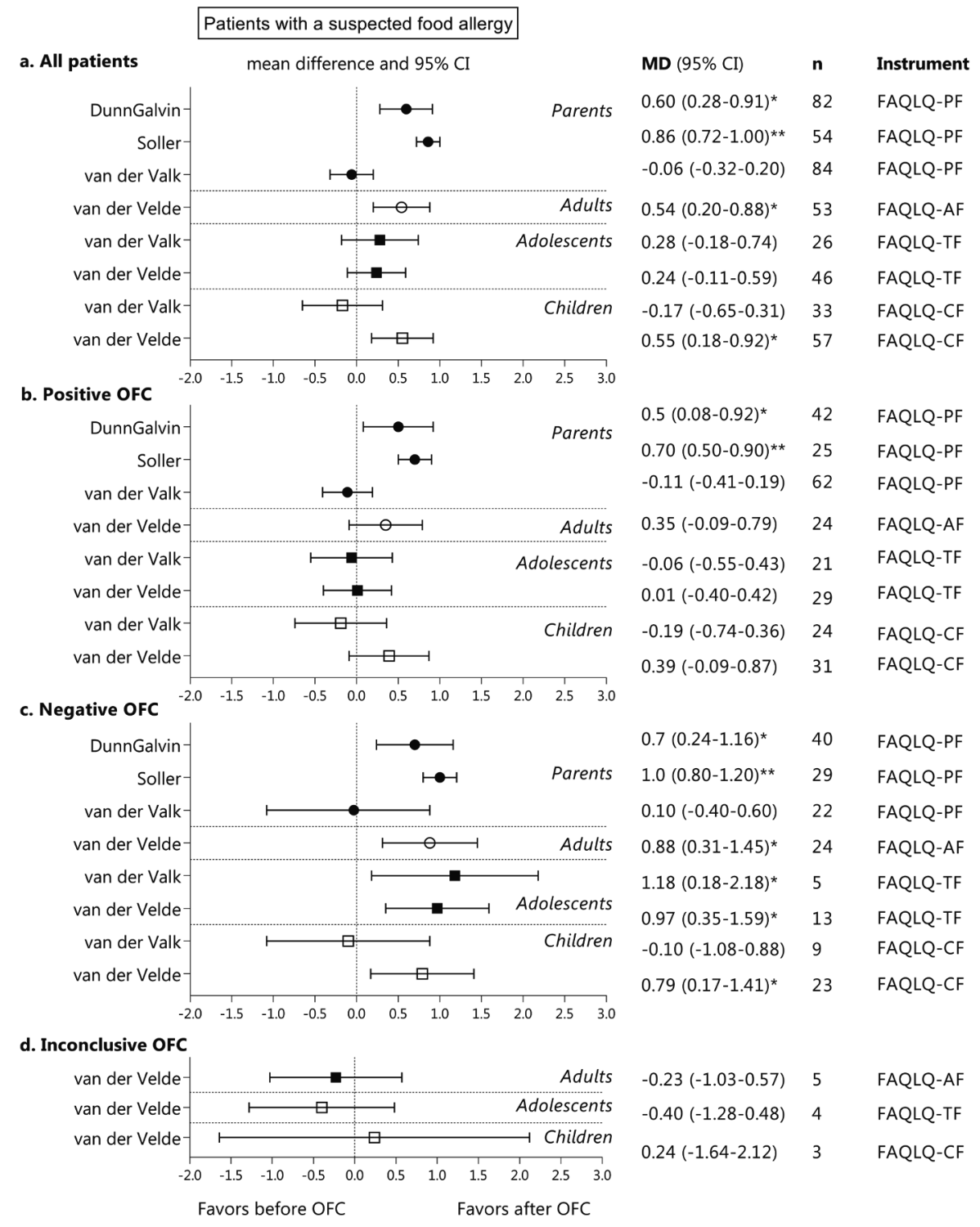

Figure 2. Health-related quality of life before and after an oral food challenge in patients with a suspected food allergy

Legend see Figure 3. 


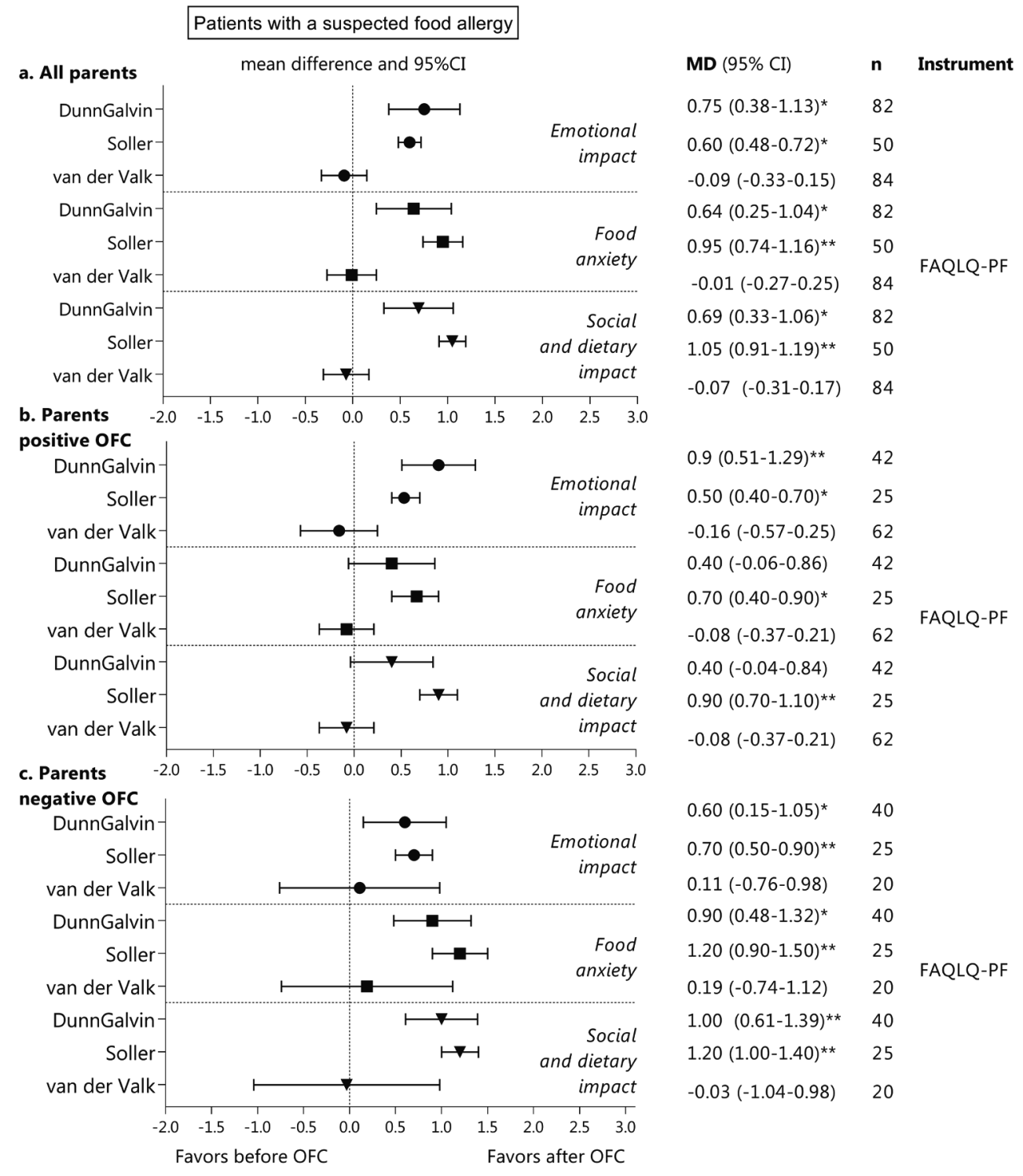

Figure 3. Domain-specific health-related quality of life before and after an oral food challenge in patients with a suspected food allergy

* Significant change in health-related quality of life.

** Significant and clinically relevant change in health-related quality of life.

Abbreviations: $\mathrm{Cl}$, confidence interval; FAQL, Food Allergy Quality of Life Questionnaire (different versions: $A F$, adult form; $C F$, child form; TF, teenager form; $P F$, parent form); $M D$, mean difference; $n$, number; OFC, oral food challenge. 


\section{Quality of life in patients with a confirmed food allergy}

In patients with a confirmed food allergy who underwent an OFC parent-reported food allergy specific HRQL and HRQL in children significantly improved after a single dose OFC in one publication (Figure 4). ${ }^{26}$ The standardized mean difference (SMD) was very large. The aim of the OFC in this publication was to assess the $\mathrm{ED}_{05}$, which is the dose that elicits an allergic reaction in $5 \%$ of the allergic subjects (Table 1). The MD and domain-specific HRQL values were not available.

The parental burden was just significantly reduced after an OFC in patients with a confirmed food allergy included in one publication. ${ }^{30}$ The effect size was small. Generic HRQL in both parents and children did not improve after an OFC (Figure 4). The domain-specific HRQL values were not available.

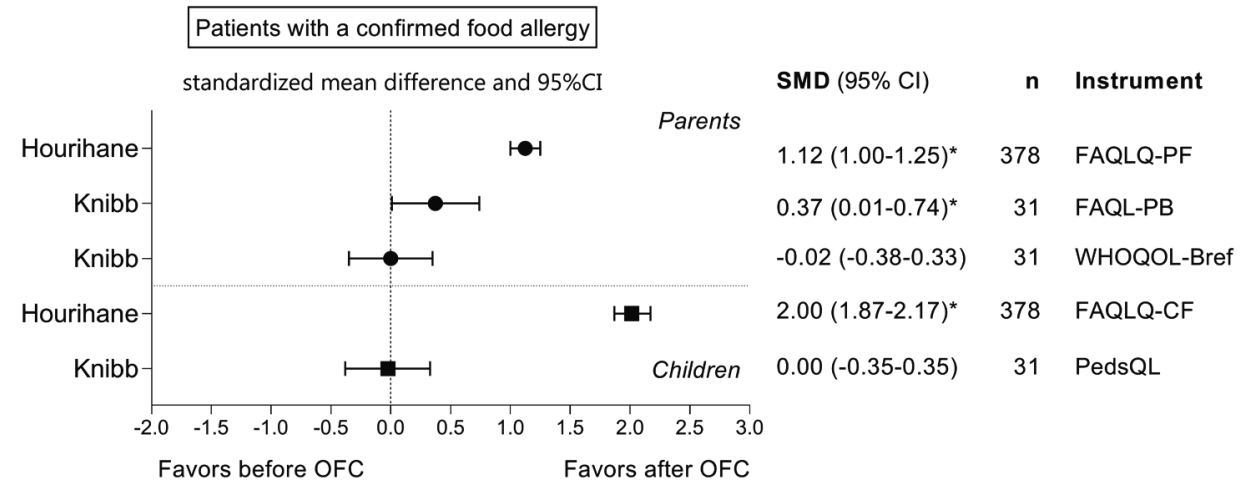

Figure 4: Health-related quality of life and the parental burden before and after an oral food challenge in patients with a confirmed food allergy

The OFC was performed to evaluate the peanut eliciting dose (Hourihane), or to assess resolution of food allergy (Knibb).

Abbreviations: $\mathrm{Cl}$, confidence interval; $F A Q L(Q)$, Food Allergy Quality of Life (Questionnaire) (different versions: CF, Child Form; PB, Parental Burden; PF, Parent Form); n, number; PedsQL ${ }^{T M}$ 4.0, Paediatric Quality of Life Inventory 4.0; SMD, standardized mean difference; WHOQOL-BREF, World Health Organization generic Quality of Life scale.

In addition to the inclusion of patients that underwent an OFC, one publication included patients that did not undergo an OFC. ${ }^{30}$ All patients who underwent an OFC were suspected of resolution of their food allergy, while the patients who did not undergo an OFC were considered food allergic by a physician (Table 1). In parents of patients that did not undergo an OFC, no significant differences in the parental burden and generic HRQL were observed between baseline and after 3-6 months (Supplemental Figure 2b). Furthermore, no significant difference was observed between the SMD in the parental burden and generic HRQL in patients that underwent an OFC compared to patients that did not undergo an OFC (Supplemental Figure 5). 
Finally, a lower parental burden (better HRQL) was observed in parents of children with a food allergy confirmed with an OFC compared to parents of children with a food allergy confirmed without an OFC in one publication (mean FAQL-PB after an OFC 1.5 (95\% CI 1.37-1.62) and mean FAQL-PB without an OFC 1.88 (95\% CI 1.791.95); $\mathrm{p}<0.0001){ }^{28}$

\section{Meta-analysis}

A meta-analysis was considered inappropriate due to the limited number of publications available, the profound differences between the included populations and the different outcome measurements (HRQL questionnaires) that were used in the included publications.

\section{Discussion}

We present the first systematic review that evaluates whether oral food challenges (OFC) for food allergy affect health-related quality of life (HRQL) in patients with a suspected or a confirmed food allergy. Our findings indicate that an OFC is associated with an improved food allergy specific HRQL. In the majority of included publications food allergy specific HRQL improved after an OFC ${ }^{18,19,26,29}$, and not in patients that did not undergo an OFC. ${ }^{19,30}$ The parental burden was just significantly reduced after an OFC, and did not change in patients that did not undergo an OFC. No information was available about whether other diagnostic interventions affect HRQL.

Our results show that an OFC is associated with a significantly improved parentreported HRQL after an OFC. ${ }^{18,26,29}$ An OFC might have a beneficial effect because the challenge procedure clarifies the severity of the food allergy, reduces anxiety as parents and patients experience the exposure to the food allergen in a controlled environment and learn how to handle in case of severe reaction in daily practice. ${ }^{31-}$ ${ }^{33}$ In addition, an accurate food allergy diagnosis might decrease uncertainty after an OFC which is confirmed by the lack of HRQL improvement in patients after an OFC with an inconclusive outcome. Food allergy specific HRQL continued to improve between two and six months after an OFC in parents included by DunnGalvin et al. but not in Soller et al. This difference might be explained by several modifying factors that varied between the publications, such as the maintenance of regular clinical contact, guided food reintroduction, and other differences in management strategies after the OFC.

It must be noted that, in contrast to the publications by DunnGalvin and Soller, parent-reported food allergy specific HRQL did not improve after an OFC in patients included by van der Valk et al. ${ }^{27}$ This discrepant result might be explained 
by differences between the populations that were studied. The children included by van der Valk et al were all suspected of having a cashew nut allergy, while the children included by DunnGalvin et al and Soller et al were suspected of other allergies like a peanut, cow's milk or hen's egg allergy. Previous research has demonstrated that the type of food allergen is associated with HRQL in children and adults. ${ }^{21,34}$ Children with a suspected cashew nut allergy might experience no improved HRQL after an OFC because cashew nut is probably easier to avoid than other food allergens such as peanut, cow's milk or hen's egg. This hypothesis is supported by the fact that the baseline HRQL value in patients included by van der Valk was much lower - indicating a better HRQL - compared to the baseline value in patients included by DunnGalvin et al. The baseline HRQL value in parents included by Soller et al. was not reported.

The publications by van der Velde et al. and Knibb et al. assessed food allergy specific HRQL in patients with and without an OFC, and observed a significantly improved HRQL and reduced parental burden in patients with an OFC but not in patients without an OFC. ${ }^{19,30}$ However, there was no significant difference between the MD in food allergy specific HRQL or the parental burden in patients that underwent an OFC compared to the patients that did not undergo an OFC. These results should be interpreted with caution because in both publications patients were not randomized to the OFC, thus confounding may have biased the results as patients in who an OFC was performed were not fully comparable to those that did not undergo an OFC (Table 1). It is not surprising that HRQL did not improve in patients on the waiting list for an OFC as these patients remain uncertain regarding their food allergic status. In the publication by Knibb et al. an OFC was only performed in patients if resolution of food allergy was considered plausible by the physician and therefore baseline HRQL was better and the parental burden was lower in patients who underwent an OFC compared to those who did not undergo an OFC. Furthermore, the patients that underwent an OFC were significantly older which might have contributed to the improvement in HRQL in this group as an older age is associated with a better HRQL. 35,36

We summarized the literature on the effect of diagnostic interventions on HRQL in patients with a suspected or a confirmed food allergy. The interpretation of our review is limited as no diagnostic randomized trials have been performed, which are needed to adequately assess the true effect of a diagnostic intervention on HRQL without bias. ${ }^{37}$ However, such a study design is hardly feasible as an OFC is the reference standard to diagnose a food allergy. In addition, we were unable to perform a meta-analysis to summarize the results of the included publications, or to analyze subgroups of patients with different characteristics, due to the limited number of eligible publications and the differences in HRQL questionnaires that were used. Furthermore, there were a limited number of eligible publications that 
satisfied inclusion criteria and the majority of the included publications were at high or moderate risk of bias. Finally, our results are based on calculations using an assumed correlation coefficient ( $r$ ) of 0.5 . However, a sensitivity analysis with a range of plausible $r$ values hardly changed our results (Supplemental Table 5).

In conclusion, we found that an oral food challenge (OFC) is associated with an improved food allergy specific health-related quality of life (HRQL) and a reduced parental burden of food allergy. Further prospective HRQL research is necessary to support the findings of our review and investigate the impact of other diagnostic interventions on HRQL.

\section{Acknowledgments}

We would like to thank Harmieke van Os-Medendorp for her statistical advice. 


\section{References}

1. Allen KJ, Koplin JJ. The Epidemiology of IgE-Mediated Food Allergy and Anaphylaxis. Immunol Allergy Clin North Am. 2012;32(1):35-50.

2. Rona RJ, Keil T, Summers $C$, et al. The prevalence of food allergy: A meta-analysis. J Allergy Clin Immunol. 2007;120(3):638-646.

3. Nwaru BI, Hickstein L, Panesar SS, et al. The epidemiology of food allergy in Europe: A systematic review and meta-analysis. Allergy. 2014;69(1):62-75.

4. Muraro A, Dubois AE, Dunngalvin A, et al. EAACI food allergy and anaphylaxis guidelines. Food allergy health-related quality of life measures. Allergy. 2014;69(7):845-853.

5. DunnGalvin A, Dubois AE, Flokstra-de Blok BM, Hourihane JO. The effects of food allergy on quality of life. Chem Immunol Allergy. 2015;101:235-252.

6. Antolín-Amérigo D, Manso L, Caminati M, et al. Quality of life in patients with food allergy. Clin Mol Allergy. 2016;14(1):4.

7. Flokstra-De Blok BMJ, Van Der Velde JL, Vlieg-Boerstra BJ, et al. Health-related quality of life of food allergic patients measured with generic and disease-specific questionnaires. Allergy. 2010;65(8):1031-1038.

8. Flokstra-de Blok B, Dubois A, Vlieg-Boerstra B, et al. Health-related quality of life of food allergic patients: comparison with the general population and other diseases. Allergy. 2010;65(2):238-244.

9. Muraro A, Werfel T, Hoffmann-Sommergruber K, et al. EAACI Food Allergy and Anaphylaxis Guidelines: Diagnosis and management of food allergy. Allergy. 2014;69(8):1008-1025.

10. Chokshi NY, Sicherer SH, Chokshi NY, Sicherer SH. Interpreting IgE sensitization tests in food allergy. Expert Rev Clin Immunol. 2016;12(4):389-403.

11. Sampson HA, Wijk G Van, Bindslev-jensen C, Sicherer S. Standardizing double-blind, placebocontrolled oral food challenges: American Academy of Allergy, Asthma \& Immunology European Academy of Allergy and Clinical Immunology PRACTALL consensus report. J Allergy Clin Immunol. 2012;130(6):1260-1274.

12. Vlieg-Boerstra BJ, Herpertz I, Pasker L, et al. Validation of novel recipes for double-blind, placebo-controlled food challenges in children and adults. Allergy Eur J Allergy Clin Immunol. 2011;66(7):948-954.

13. Kansen H, Erp F Van, Le T, Meijer Y, Ent C Van Der. PROSPERO International prospective register of systematic reviews A systematic review of the cost-effectiveness and impact on quality of life of diagnostic testing in patients with suspected food allergy. 2017:1-6.

14. Moher D, Liberati A, Tetzlaff J, Altmann D. The PRISMA Group. Preferred Reporting Items for Systematic Reviews and Meta-Analyses: The PRISMA Statement. Ann Intern Med. 2009;151(4):264-269.

15. Ouzzani M. Rayyan — a web and mobile app for systematic reviews. Syst Rev. 2016;5:1-10.

16. Falagas ME, Pitsouni EI, Malietzis GA, Pappas G. Comparison of PubMed, Scopus, Web of Science, and Google Scholar: strengths and weaknesses. FASEB J. 2017;22(2):338-342.

17. Hayden JA, Coté $P$, Bombardier $C$. Evaluation of the quality of prognosis studies in systematic reviews. Ann Intern Med. 2006;144(6):427-437.

18. Dunngalvin A, Cullinane C, Daly DA, Blok BMJF, Dubois AEJ, Hourihane JOB. Longitudinal validity and responsiveness of the Food Allergy Quality of Life Questionnaire - Parent Form in children $0-12$ years following positive and negative food challenges. Clin Exp Allergy. 2010:476-485. 
19. Van Der Velde JL, Flokstra-De Blok BMJ, De Groot H, et al. Food allergy-related quality of life after double-blind, placebo-controlled food challenges in adults, adolescents, and children. J Allergy Clin Immunol. 2012;130(5):1136-1143.e2.

20. Cohen BL, Noone S, Muñoz-Furlong A, Sicherer SH. Development of a questionnaire to measure quality of life in families with a child with food allergy. I Allergy Clin Immunol. 2004;114(5):1159-1163.

21. Saleh-Langenberg J, Goossens NJ, Flokstra-De Blok BMJ, et al. Predictors of health-related quality of life of European food-allergic patients. Allergy. 2015;70(6):616-624.

22. Borenstein $M$, Hedges L V, Higgins JPT, Rothstein HR. Effect Sizes Based on Means. In: Introduction to Meta-Analysis. Chichester: John Wiley \& Sons Ltd; 2009:21-32.

23. Revicki D, Hays RD, Cella D, Sloan J. Recommended methods for determining responsiveness and minimally important differences for patient-reported outcomes. J Clin Epidemiol. 2008;61(2):102-109.

24. Cohen J. Statistical Power Analysis for the Behavioral Sciences. New York: Academic Press; 1977.

25. Haidich A. Meta-analysis in medical research. Hippokratia. 2010;14(Suppl 1):29-37.

26. Hourihane JOB, Allen KJ, Shreffler WG, et al. Peanut Allergen Threshold Study (PATS): Novel single-dose oral food challenge study to validate eliciting doses in children with peanut allergy. J Allergy Clin Immunol. 2017;139(5):1583-1590.

27. van der Valk JPM, Gerth van Wijk R, Flokstra-de Blok BMJ, et al. No difference in health-related quality of life, after a food challenge with cashew nut in children participating in a clinical trial. Pediatr Allergy Immunol. 2016;27(8):812-817.

28. Franxman TJ, Howe L, Teich E, Greenhawt MJ. Oral Food Challenge and Food Allergy Quality of Life in Caregivers of Children with Food Allergy. J Allergy Clin Immunol Pract. 2014;3(1):50-56.

29. Soller L, Hourihane J, Dunngalvin A. The impact of oral food challenge tests on food allergy health-related quality of life. Allergy. 2014;69(9):1255-1257.

30. Knibb RC, Ibrahim NF, Stiefel G, et al. The psychological impact of diagnostic food challenges to confirm the resolution of peanut or tree nut allergy. Clin Exp Allergy. 2012;42(3):451-459.

31. Zijlstra WT, Flinterman $A E$, Soeters $L$, et al. Parental anxiety before and after food challenges in children with suspected peanut and hazelnut allergy. Pediatr Allergy Immunol. 2010;21:439-445.

32. Nguyen $M$, Wainstein BK, Hu W, Ziegler JB. Parental satisfaction with oral peanut food challenges; perception of outcomes and impact on management of peanut allergy. Pediatr Allergy Immunol. 2010;21(8):1119-1126.

33. Kemp AS, Allen CW, Campbell DE. Parental perceptions in egg allergy: Does egg challenge make a difference? Pediatr Allergy Immunol. 2009;20(7):648-653.

34. Howe L, Franxman $T$, Teich $E$, Greenhawt M. What affects quality of life among caregivers of food-allergic children? Ann Allergy Asthma Immunol. 2014;113(1):69-74.e2.

35. Marklund B, Ahlstedt S, Nordström G. Health-related quality of life in food hypersensitive schoolchildren and their families: parents' perceptions. Health Qual Life Outcomes. 2006;4:48.

36. Wassenberg J, Cochard MM, Dunngalvin A, et al. Parent perceived quality of life is agedependent in children with food allergy. Pediatr Allergy Immunol. 2012;23(5):412-419.

37. Rodger M, Ramsay $\mathrm{T}$, Fergusson D. Diagnostic randomized controlled trials: the final frontier. Trials. 2012;13(1):1. 


\section{Supplemental information}

\section{Supplemental 1: Search strategy MEDLINE}

Date search $6^{\text {th }}$ of July 2017

1. foodallerg*[Title/Abstract]

2. "food allergy" [Title/Abstract]

3. "food allergies"[Title/Abstract]

4. "food hypersensitivity"[Title/Abstract]

5. "food hypersensitivities"[Title/Abstract]

6. peanut*[Title/Abstract]

7. Arachis[Title/Abstract]

8. milk*[Title/Abstract]

9. egg*[Title/Abstract]

10. fish*[Title/Abstract]

11. shellfish*[Title/Abstract]

12. "tree nut"[Title/Abstract]

13. hazelnut*[Title/Abstract]

14. walnut*[Title/Abstract]

15. macadamia*[Title/Abstract]

16. cashew*[Title/Abstract]

17. pistachio*[Title/Abstract]

18. wheat*[Title/Abstract]

19. sesame*[Title/Abstract]

20. soy*[Title/Abstract]

21. food hypersensitivity[MeSH]

22. Arachis $[\mathrm{MeSH}])$

23. 1 or 2 or 3 or 4 or 5 or 6 or 7 or 8 or 9 or 10 or 11 or 12 or 13 or 14 or 15 or 16 or 17 or 18 or 19 or 20 or 21 or 22

24. (CRD[Title/Abstract]

25. Component*[Title/Abstract]

26. Allergen*[Title/Abstract]

27. Ara[Title/Abstract]

28. Extract[Title/Abstract]

29. IgE[Title/Abstract]

30. slgE[Title/Abstract]

31. "skin prick test"[Title/Abstract]

32. "skin prick testing" [Title/Abstract]

33. SPT[Title/Abstract]

34. challenge[Title/Abstract]

35. DBPCFC[Title/Abstract]

36. double-blind[Title/Abstract]

37. "double blind"[Title/Abstract]

38. placebo-controlled [Title/Abstract]

39. "placebo controlled"[Title/Abstract]

40. OFC[Title/Abstract]

41. Oral[Title/Abstract])

42. 24 or 25 or 26 or 27 or 28 or 29 or 30 or 31 or 32 or 33 or 34 or 35 or 36 or 37 or 38 or 39 or 40 or 41

43. "quality of life"[Title/Abstract]

44. "quality-of-life"[Title/Abstract]

45. QoL[Title/Abstract]

46. HRQL[Title/Abstract]
47. HRQoL[Title/Abstract]

48. FAQLQ*[Title/Abstract]

49. quality of life[MeSH]

50. EQ-5D[Title/Abstract]

51. EQ5D[Title/Abstract]

52. "EQ 5D"[Title/Abstract]

53. EuroQol-5D[Title/Abstract]

54. “EuroQol 5D" [Title/Abstract]

55. HUI[Title/Abstract]

56. "Health Utilities Index"[Title/Abstract]

57. SF-6D[Title/Abstract]

58. "SF 6D"[Title/Abstract]

59. "short form-6D" [Title/Abstract]

60. SF-36[Title/Abstract]

61. "SF 36 "[Title/Abstract]

62. "short form 36 " [Title/Abstract]

63. SF-12[Title/Abstract]

64. "SF 12" [Title/Abstract]

65. "short form 12"[Title/Abstract]

66. "short form health survey"[Title/Abstract]

67. "State-Trait Anxiety Inventory"[Title/Abstract]

68. STAI[Title/Abstract])

69. 43 or 44 or 45 or 46 or 47 or 48 or 49 or 50 or 51 or 52 or 53 or 54 or 55 or 56 or 57 or 58 or 59 or 60 or 61 or 62 or 63 or 64 or 65 or 66 or 67 or 68

70. 23 and 42 and 69

\section{EMBASE}

Date search $6^{\text {th }}$ of July 2017

1. foodallerg*:ab,ti

2. 'food allergy':ab,ti

3. 'food allergies':ab,ti

4. 'food hypersensitivity':ab,ti

5. 'food hypersensitivities':ab,ti

6. peanut*:ab,ti

7. Arachis:ab,ti

8. milk:ab,ti

9. egg*:ab,ti

10. fish*:ab,ti

11. shellfish*:ab,ti

12. 'tree nut':ab,ti

13. hazelnut*:ab,ti

14. walnut*:ab,ti

15. macadamia*:ab,ti

16. cashew*:ab,ti

17. pistachio*:ab,ti

18. wheat*:ab,ti

19. sesame*:ab,ti

20. soy*:ab,ti

21. 'food allergy'/exp 
22. 'arachis'/exp)

23. 1 or 2 or 3 or 4 or 5 or 6 or 7 or 8 or 9 or 10 or 11 or 12 or 13 or 14 or 15 or 16 or 17 or 18 or 19 or 20 or 21 or 22

24. crd:ab,ti

25. component*:ab,ti

26. allergen*:ab,ti

27. ara:ab,ti

28. extract:ab,ti

29. ige:ab,ti

30. sige:ab,ti

31. 'skin prick test':ab,ti

32. 'skin prick testing':ab,ti

33. spt:ab,ti

34. challenge:ab,ti

35. dbpcfc:ab,ti

36. 'double-blind':ab,ti

37. 'double blind':ab,ti

38. 'placebo-controlled':ab,ti

39. 'placebo controlled':ab,ti

40. ofc:ab,ti

41. oral:ab,ti

42. 24 or 25 or 26 or 27 or 28 or 29 or 30 or 31 or 32 or 33 or 34 or 35 or 36 or 37 or 38 or 39 or 40 or 41

43. 'quality of life':ab,ti

44. 'quality-of-life':ab,ti

45. qol:ab,ti

46. hrql:ab,ti

47. hrqol:ab,ti

48. faqlq*:ab,ti

49. 'quality of life'/exp

50. 'quality of life':ab,ti

51. EQ-5D:ab,ti

52. EQ5D:ab,ti

53. 'EQ 5D':ab,ti

54. EuroQol-5D:ab,ti

55. 'EuroQol 5D':ab,ti

56. HUI:ab,ti

57. 'Health Utilities Index':ab,ti

58. SF-6D:ab,ti

59. 'SF $6 D$ ':ab,ti

60. 'short form-6D':ab,ti

61. SF-36:ab,ti

62. 'SF 36 ':ab,ti

63. 'short form 36 ':ab,ti

64. SF-12:ab,ti

65. 'SF 12 ':ab,ti

66. 'short form 12 ':ab,ti

67. 'short form health survey':ab,ti

68. 'State-Trait Anxiety Inventory':ab,ti

69. STAl:ab,ti

70. 43 or 44 or 45 or 46 or 47 or 48 or 49 or 50 or 51 or 52 or 53 or 54 or 55 or 56 or 57 or 58 or 59 or 60 or 61 or 62 or 63 or 64 or 65 or 66 or 67 or 68 or 69

71. 23 and 42 and 70 and [embase]/lim not [medline]/lim

\section{Cochrane Library}

Date search $6^{\text {th }}$ of July 2017

1. foodallerg*:ab,ti,kw

2. "food allergy":ab,ti,kw

3. "food allergies":ab,ti,kw

4. "food hypersensitivity":ab,ti,kw

5. "food hypersensitivities":ab,ti,kw

6. peanut*:ab,ti,kw

7. Arachis:ab,ti,kw

8. milk:ab,ti,kw

9. egg*:ab,ti,kw

10. fish*:ab,ti,kw

11. shellfish*:ab,ti,kw

12. "tree nut":ab,ti,kw

13. hazelnut*:ab,ti,kw

14. walnut*:ab,ti,kw

15. macadamia*:ab,ti, kw

16. cashew*:ab,ti,kw

17. pistachio*:ab,ti,kw

18. wheat*:ab,ti,kw

19. sesame*:ab,ti,kw

20. soy*:ab,ti,kw)

21. 1 or 2 or 3 or 4 or 5 or 6 or 7 or 8 or 9 or 10 or 11 or 12 or 13 or 14 or 15 or 16 or 17 or 18 or 19 or 20

22. CRD:ab,ti,kw

23. Component*:ab,ti,kw

24. Allergen*:ab,ti,kw

25. Ara:ab,ti,kw

26. Extract:ab,ti,kw

27. ige:ab,ti,kw

28. sige:ab,ti,kw

29. "skin prick test":ab,ti,kw

30. "skin prick testing":ab,ti,kw

31. spt:ab,ti,kw

32. challenge:ab,ti,kw

33. dbpcfc:ab,ti,kw

34. "double-blind":ab,ti,kw

35. 'double blind':ab,ti,kw

36. "placebo-controlled":ab,ti,kw

37. "placebo controlled":ab,ti,kw

38. ofc:ab,ti,kw

39. oral:ab,ti,kw

40. 22 or 23 or 24 or 25 or 27 or 27 or 28 or 29 or 30 or 31 or 32 or 33 or 34 or 35 or 36 or 37 or 38 or 39

41. "quality of life":ti,ab,kw

42. "quality-of-life":ti,ab,kw

43. QoL:ti,ab,kw

44. HRQL:ti,ab,kw

45. HRQoL:ti,ab,kw

46. FAQLQ*:ti,ab,kw

47. EQ-5D:ti,ab,kw

48. EQ5D:ti,ab,kw

49. "EQ 5D" :ti,ab,kw

50. EuroQol-5D:ti,ab,kw 
51. “EuroQol 5D” :ti,ab,kw

52. HUI:ti,ab,kw

53. "Health Utilities Index" :ti,ab,kw

54. SF-6D:ti,ab,kw

55. "SF 6D" :ti,ab,kw

56. "short form-6D" :ti,ab,kw

57. SF-36:ti,ab,kw

58. "SF 36 " :ti,ab,kw

59. "short form 36 " :ti,ab, kw

60. SF-12:ti,ab,kw

61. "SF 12" :ti,ab,kw

62. "short form 12" :ti,ab,kw

63. "short form health survey" :ti,ab,kw

64. "State-Trait Anxiety Inventory":ti,ab,kw

65. STAl:ti,ab,kw

66. 41 or 42 or 43 or 44 or 45 or 46 or 47 or 48 or 49 or 50 or 51 or 52 or 53 or 54 or 55 or 56 or 57 or 58 or 59 or 60 or 61 or 62 or 63 or 64 or 65

67. 21 and 40 and 66

\section{CINAHL}

Date search $6^{\text {th }}$ of July 2017

1. foodallergy

2. foodallergies

3. foodallergen

4. "food allergy"

5. "food allergies"

6. peanut

7. milk

8. egg

9. fish

10. shellfish

11. "tree nut"

12. hazelnut

13. walnut

14. macadamia

15. cashew

16. pistachio

17. wheat

18. sesame

19. soy

20. "food hypersensitivity"

21. Arachis

22. (MH "Food Hypersensitivity")

23. 1 or 2 or 3 or 4 or 5 or 6 or 7 or 8 or 9 or 10 or 11 or 12 or 13 or 14 or 15 or 16 or 17 or 18 or 19 or 20 or 21 or 22

24. CRD

25. Component

26. Allergen

27. Ara

28. Extract

29. $\operatorname{IgE}$

30. slgE

31. "skin prick test"

32. "skin prick testing"

33. SPT
34. challenge

35. DBPCFC

36. double-blind

37. "double blind"

38. placebo-controlled

39. "placebo controlled"

40. OFC

41. Oral

42. 24 or 24 or 26 or 27 or 28 or 29 or 30 or 31 or 32 or 33 or 34 or 35 or 36 or 37 or 38 or 39 or 40 or 41

43. "quality of life"

44. "quality-of-life"

45. QoL OR HRQL

46. HRQoL

47. FAQLQ

48. (MH "Quality of Life+")

49. EQ-5D

50. EQ5D

51. "EQ 5D"

52. EuroQol-5D

53. "EuroQol 5D"

54. HUI

55. "Health Utilities Index"

56. SF-6D

57. "SF 6D"

58. "short form-6D"

59. SF-36

60. "SF 36"

61. "short form 36"

62. SF-12

63. "SF 12"

64. "short form 12"

65. "short form health survey"

66. "State-Trait Anxiety Inventory"

67. STAI

68. 43 or 44 or 45 or 46 or 47 or 48 or 49 or 50 or 51 or 52 or 53 or 54 or 55 or 56 or 57 or 58 or 59 or 60 or 61 or 62 or 63 or 64 or 65 or 66 or 67

69. 23 and 42 and 68 


\section{Supplemental figures}

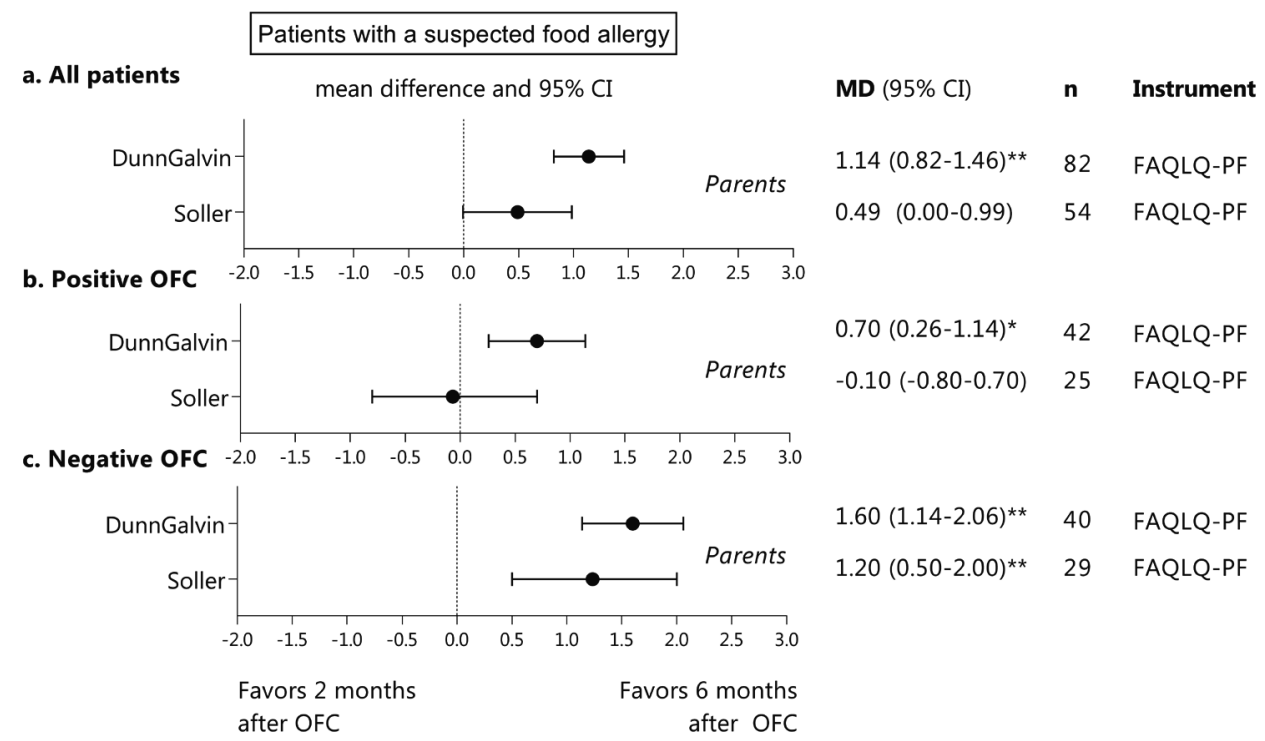

Supplemental Figure 1. Health-related quality of life after an oral food challenge in patients with a suspected food allergy 2 and 6 months after an OFC

* significant change in HRQL.

** significant and clinically relevant change in HRQL.

Abbreviations: Cl, confidence interval; FAQLQ-PF, Food Allergy Quality of Life Questionnaire-Parent Form; $M D$, mean difference; $n$, number; OFC, oral food challenge. 


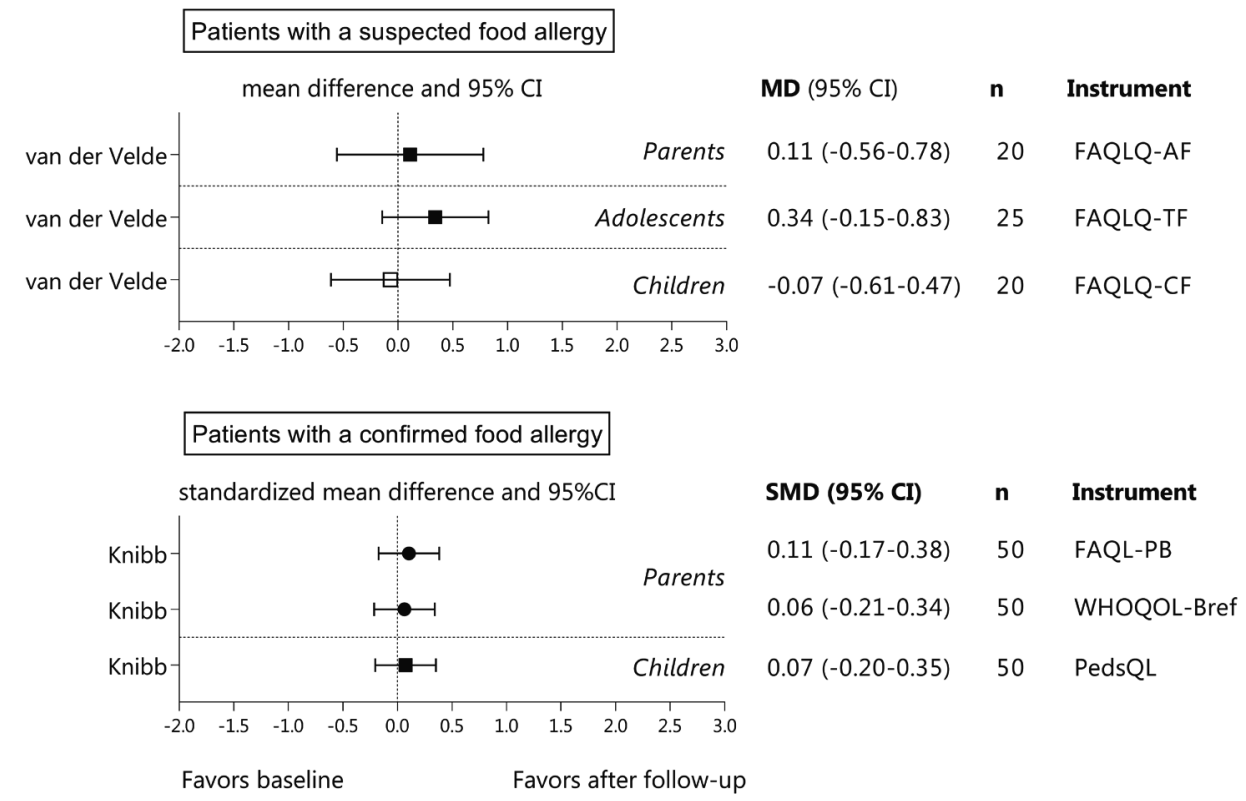

Supplemental Figure 2. Health related quality of life and parental burden in patients without an OFC at baseline and after follow-up

Abbreviations: $C l$, confidence interval; $F A Q L(Q)$, Food Allergy Quality of Life (Questionnaire) (different versions: AF, Adult Form; CF, Child Form; PB, Parental Burden; TF, Teen Form); MD, mean difference; $n$, number; $S M D$, standardized mean difference.

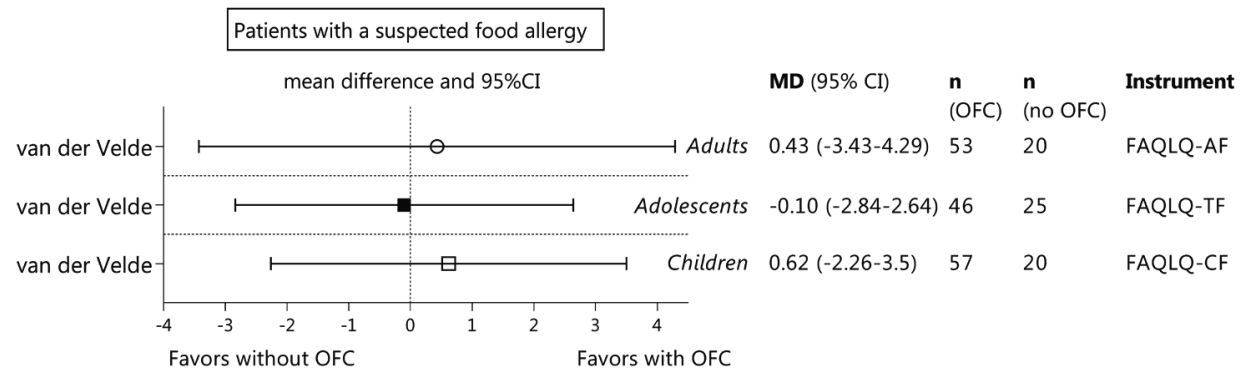

Supplemental Figure 3. Health-related quality of life with or without an oral food challenge in patients with a suspected food allergy

An OFC was performed in patients with a suspected food allergy. An OFC was not performed in patients with a suspected food allergy on the waiting list for an OFC, or in patients considered food allergic by a physician.

* significant change in HRQL.

Abbreviations: $\mathrm{Cl}, \mathrm{Cl}$, confidence interval; FAQLQ, Food Allergy Quality of Life Questionnaire (different versions: AF, Adult Form; CF, Child Form; TF, Teen Form); MD, mean difference; $n$, number; OFC, oral food challenge. 


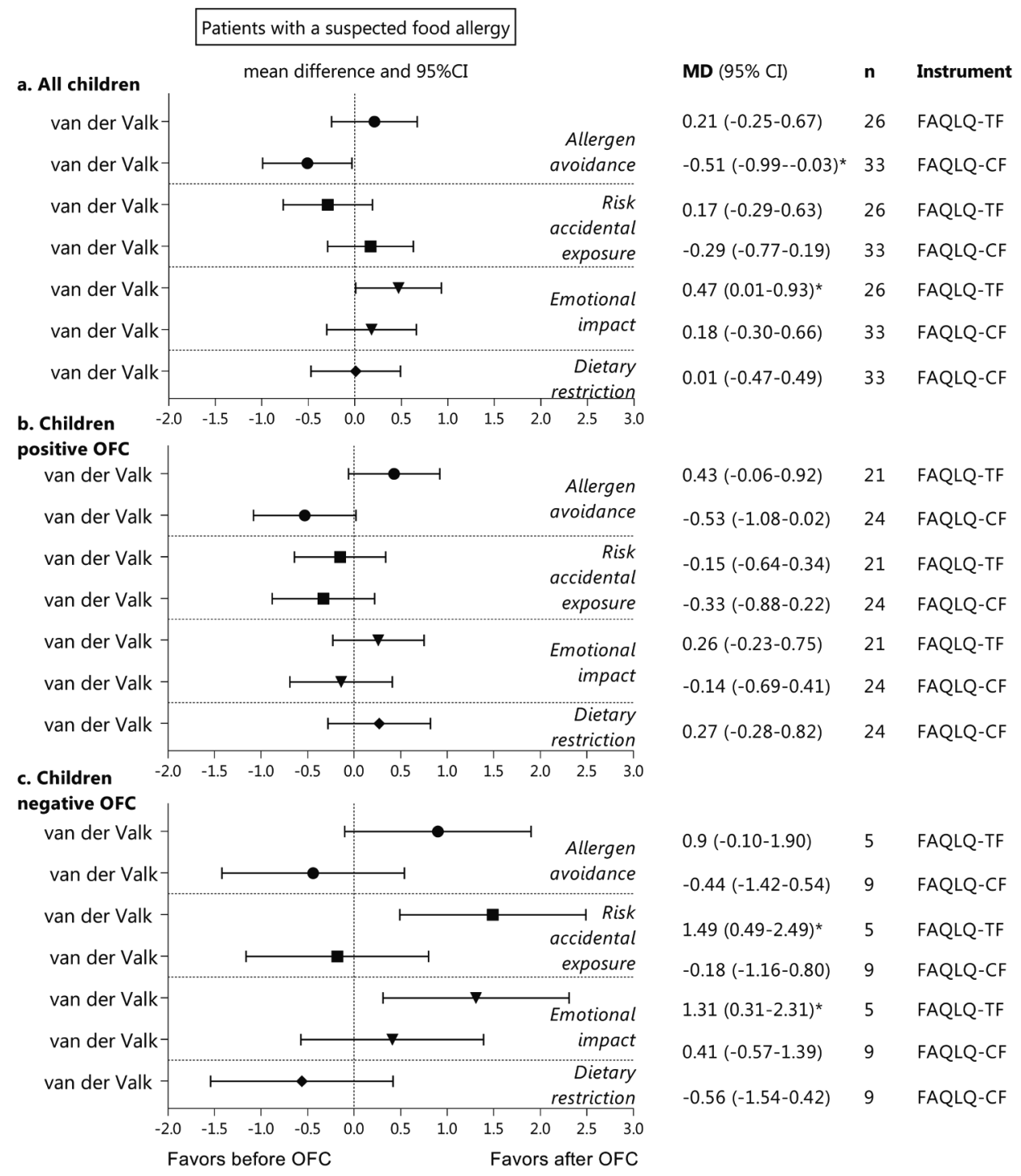

Supplemental Figure 4. Domain specific health-related quality of life before and after an oral food challenge in children and adolescents with a suspected food allergy * significant change in HRQL.

Abbreviations: $\mathrm{Cl}, \mathrm{Cl}$, confidence interval; FAQLQ, Food Allergy Quality of Life Questionnaire (different versions: CF, Child Form; TF, Teen Form); HRQL, health-related quality of life; MD, mean difference; $n$, number; OFC, oral food challenge. 


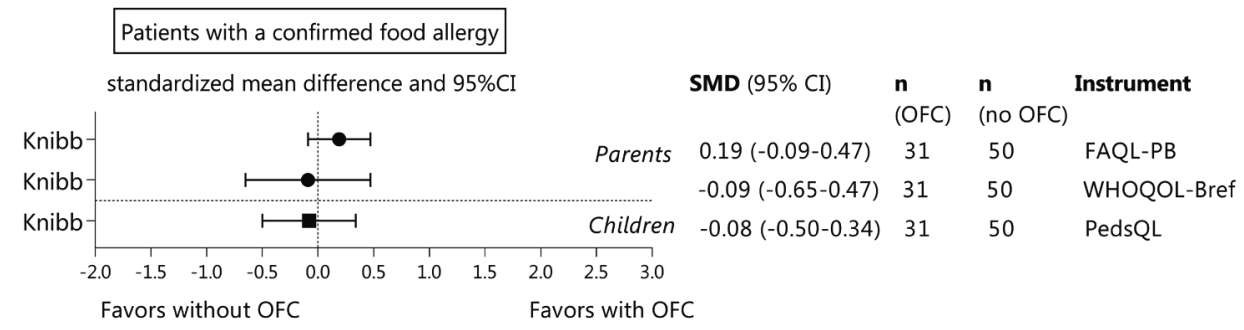

Supplemental Figure 5. Health-related quality of life and the parental burden with or without an oral food challenge in patients with a confirmed food allergy

An OFC was performed in patients with a confirmed food allergy to evaluate resolution of food allergy. An OFC was not performed in patients considered food allergic by a physician.

Abbreviations: Cl, confidence interval; FAQL-PB, Food Allergy Quality of Life-Parental Burden; MD, mean difference; n, number; PedsQL ${ }^{T M}$ 4.0, Paediatric Quality of Life Inventory 4.0; SMD, standardized mean difference; WHOQOL-BREF, World Health Organization generic Quality of Life scale. 


\section{Supplemental tables}

Supplemental Table 1. Reasons for exclusion

\begin{tabular}{|c|c|c|c|c|}
\hline \multicolumn{5}{|c|}{ Reasons for exclusion of publications identified through database searching (until 06-07-2017) } \\
\hline First author & Year & $\mathbf{n}$ & $\begin{array}{l}\text { Category of } \\
\text { exclusion }\end{array}$ & Reason for exclusion \\
\hline Soller $^{1}$ & 2017 & 1 & Intervention & No diagnostic intervention investigated \\
\hline Westwood $^{2}$ & 2016 & 1 & Study design & Review \\
\hline Uehara $^{3}$ & 2016 & 1 & Outcome & Quality of life questionnaire not validated \\
\hline Danchin $^{4}$ & 2016 & 1 & Intervention & No diagnostic intervention investigated \\
\hline Greenhawt $^{5}$ & 2016 & 1 & Outcome & Quality of life questionnaire not validated \\
\hline Kajornrattana $^{6}$ & 2016 & 1 & Intervention & No diagnostic intervention investigated \\
\hline Couch $^{7}$ & 2016 & 1 & Outcome & $\begin{array}{l}\text { Timing of questionnaire (not before and after } \\
\text { intervention) }\end{array}$ \\
\hline Protudjer $^{8}$ & 2015 & 1 & Intervention & No diagnostic intervention investigated \\
\hline Venter $^{9}$ & 2015 & 1 & Intervention & No diagnostic intervention investigated \\
\hline Soller ${ }^{1}$ & 2014 & 1 & Intervention & No diagnostic intervention investigated \\
\hline Howe $^{10}$ & 2014 & 1 & Intervention & No diagnostic intervention investigated \\
\hline $\mathrm{Le}^{11}$ & 2013 & 1 & Intervention & No diagnostic intervention investigated \\
\hline Zurzolo $^{12}$ & 2013 & 4 & Study design & Study protocol \\
\hline Knibb $^{13}$ & 2013 & 2 & Intervention & No diagnostic intervention investigated \\
\hline Brown $^{14}$ & 2012 & 1 & Population & Tolerant patients (after negative oral food challenge) \\
\hline Finger ${ }^{15}$ & 2012 & 1 & Outcome & $\begin{array}{l}\text { Timing of questionnaire (not before and after } \\
\text { intervention) }\end{array}$ \\
\hline Kirste $^{16}$ & 2012 & 1 & Study design & $\begin{array}{l}\text { Conference abstract, no further details could be retrieved } \\
\text { either as full text publication or after contacting study } \\
\text { authors }\end{array}$ \\
\hline Dubois $^{17}$ & 2011 & 1 & Study design & Invited speaker presentation \\
\hline Rotiroti $^{18}$ & 2011 & 1 & Outcome & Quality of life questionnaire not validated \\
\hline Paterakis $^{19}$ & 2011 & 1 & Outcome & $\begin{array}{l}\text { No quality of life questionnaire (State-Trait Anxiety } \\
\text { Inventory) }\end{array}$ \\
\hline Velde, van der ${ }^{20}$ & 2010 & 2 & Outcome & $\begin{array}{l}\text { Quality of life questionnaire used with informed and blind } \\
\text { administration }\end{array}$ \\
\hline Flammarion $^{21}$ & 2009 & 1 & Population & Tolerant patients (after negative oral food challenge) \\
\hline Sohi $^{22}$ & 2009 & 1 & Population & Tolerant patients (after negative oral food challenge) \\
\hline Zijlstra ${ }^{23}$ & 2009 & 1 & Outcome & $\begin{array}{l}\text { No quality of life questionnaire (State-Trait Anxiety } \\
\text { Inventory) }\end{array}$ \\
\hline Ostblom ${ }^{24}$ & 2008 & 1 & Intervention & No diagnostic intervention investigated \\
\hline
\end{tabular}


Supplemental Table 1. Reasons for exclusion (continued)

\begin{tabular}{|c|c|c|c|c|}
\hline \multicolumn{5}{|c|}{$\begin{array}{l}\text { Reasons for exclusion of publications identified through citations analysis of all full text screened } \\
\text { publications (until 06-07-2017) }\end{array}$} \\
\hline First author & Year & $\mathbf{n}$ & $\begin{array}{l}\text { Category of } \\
\text { exclusion }\end{array}$ & Reason for exclusion \\
\hline Polloni $i^{25}$ & 2017 & 1 & Outcome & $\begin{array}{l}\text { No quality of life questionnaire (State-Trait Anxiety } \\
\text { Inventory) }\end{array}$ \\
\hline Valk, van der ${ }^{26}$ & 2016 & 1 & Duplicate & Already included in systematic review \\
\hline Danchin $^{4}$ & 2016 & 1 & Duplicate & Excluded during full text screening of database search \\
\hline Davis $^{27}$ & 2015 & 1 & Outcome & Quality of life questionnaire not validated \\
\hline Venter $^{9}$ & 2015 & 1 & Duplicate & Excluded during data analysis \\
\hline Franxman ${ }^{28}$ & 2015 & 1 & Duplicate & Already included in systematic review \\
\hline Protudjer $^{8}$ & 2015 & 1 & Duplicate & Excluded during full text screening of database search \\
\hline Venter $^{9}$ & 2015 & 1 & Duplicate & Already included in systematic review \\
\hline Soller ${ }^{29}$ & 2014 & 1 & Duplicate & Already included in systematic review \\
\hline Reese & 2013 & 1 & Study design & Editorial \\
\hline Herbert ${ }^{30}$ & 2013 & 1 & Outcome & $\begin{array}{l}\text { No quality of life questionnaire (multiple anxiety } \\
\text { questionnaires) }\end{array}$ \\
\hline $\mathrm{Knibb}^{31}$ & 2013 & 1 & Outcome & $\begin{array}{l}\text { No quality of life questionnaire (Hospital Anxiety and } \\
\text { Depression Scale) }\end{array}$ \\
\hline Velde, van der ${ }^{32}$ & 2012 & 2 & Duplicate & Already included in systematic review \\
\hline Knibb $^{33}$ & 2012 & 2 & Duplicate & Already included in systematic review \\
\hline Flammarion $^{21}$ & 2010 & 1 & Duplicate & Excluded during full text screening of database search \\
\hline Kemp $^{34}$ & 2009 & 1 & Outcome & Quality of life questionnaire not validated \\
\hline
\end{tabular}


Supplemental Table 2. Inclusion and exclusion criteria

\begin{tabular}{|c|c|c|}
\hline \multicolumn{2}{|c|}{ Inclusion criteria } & Exclusion criteria \\
\hline $\begin{array}{l}\text { Study } \\
\text { design }\end{array}$ & $\begin{array}{l}\text { - Studies in Dutch, English, German, } \\
\text { French or Spanish }\end{array}$ & $\begin{array}{l}\text { - } \text { Systematic reviews } \\
\text { - } \text { Meta-analysis } \\
\text { - } \text { Research protocols } \\
\text { - } \quad \text { Editorials }\end{array}$ \\
\hline Population & $\begin{array}{ll}\text { - } & \text { All age } \\
\text { - } & \text { Suspected or confirmed food allergy } \\
\text { - } & \text { Any setting (primary, secondary and } \\
\text { tertiary care) }\end{array}$ & $\begin{array}{l}\text { - } \quad \text { Animal studies } \\
\text { - } \quad \text { Only non-allergic } \\
\text { patients included }\end{array}$ \\
\hline Intervention & $\begin{array}{l}\text { - } \quad \text { Specific IgE using allergen extract } \\
\text { - } \quad \text { Skin prick testing } \\
\text { - } \quad \text { Oral food challenges (double blind } \\
\text { placebo controlled food challenges } \\
\text { and open food challenges) } \\
\text { - Component resolved diagnostics }\end{array}$ & \\
\hline Outcome & $\begin{array}{l}\text { - Changes in disease-specific or generic } \\
\text { quality of life using a validated } \\
\text { instrument before and after } \\
\text { diagnostic intervention. } \\
\text { Difference in disease-specific or } \\
\text { generic quality of life using a } \\
\text { validated instrument in patients with } \\
\text { or without diagnostic intervention }\end{array}$ & $\begin{array}{l}\text { - No validated disease- } \\
\text { specific or generic } \\
\text { quality of life instrument } \\
\text { used }\end{array}$ \\
\hline
\end{tabular}


Supplemental Table 3a. Criteria for quality assessment Modified version of the Quality in Prognostic Studies (QUIPS) too ${ }^{35}$

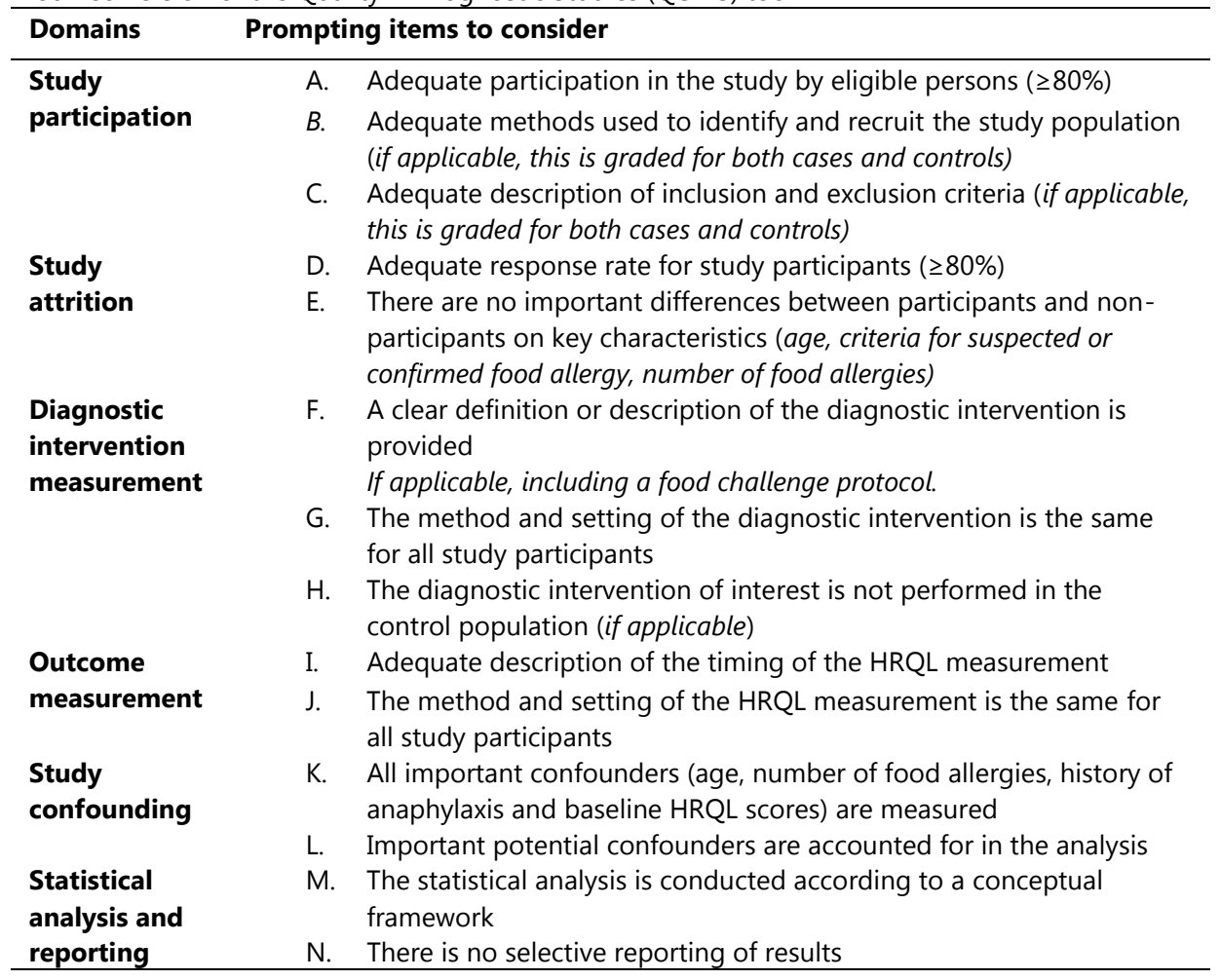

Supplemental Table $\mathbf{3 b}$. Grading of quality assessment

\begin{tabular}{llll}
\hline & High risk of bias & $\begin{array}{l}\text { Moderate risk } \\
\text { of bias }\end{array}$ & Low risk of bias \\
\hline $\begin{array}{l}\text { Total risk of bias } \\
\text { per domain in } \\
\text { one publication }\end{array}$ & $\begin{array}{l}\geq 50 \% \text { of items 'no' or } \\
\text { 'unsure', } 0 \% \text { 'yes', and } \\
\leq 50 \% \text { of items 'partial' }\end{array}$ & $\begin{array}{l}\geq 50 \% \text { of items 'yes', } 0 \% \\
\text { 'no' or 'unsure', and } \\
\leq 50 \% \text { of items 'partial' }\end{array}$ \\
$\begin{array}{l}\text { Total risk of bias } \\
\text { in one } \\
\text { publication }\end{array}$ & $\begin{array}{l}\geq 25 \% \text { of domains at high } \\
\text { risk of bias or } \geq 66 \% \text { of } \\
\text { domains at moderate or }\end{array}$ & $\begin{array}{l}\text { No low or high } \\
\text { risk of bias }\end{array}$ & $\begin{array}{l}\geq 66 \% \text { of domains } \\
\text { considered at low risk of } \\
\text { bias and } 0 \% \text { high }\end{array}$ \\
$\begin{array}{l}\text { Total risk of bias } \\
\text { per domain in all } \\
\text { publications }\end{array}$ & $\begin{array}{l}\geq 25 \% \text { of publications at } \\
\text { high risk of bias for this } \\
\text { domain or } \geq 66 \% \text { of }\end{array}$ & & $\geq 66 \%$ of publications at \\
& $\begin{array}{l}\text { publications at moderate } \\
\text { or high risk }\end{array}$ & low risk of bias and $0 \%$ \\
& & high \\
\hline
\end{tabular}


Supplemental Table 4. Risk of bias assessment

\begin{tabular}{|c|c|c|}
\hline \multicolumn{3}{|l|}{ Hourihane $2017^{36}$} \\
\hline Study participation & & $\mathrm{L}$ \\
\hline $\begin{array}{l}\text { Method used to identify } \\
\text { population (cases) }\end{array}$ & Consecutive enrollment & yes \\
\hline $\begin{array}{l}\text { Method used to identify } \\
\text { population (controls) }\end{array}$ & NA & \\
\hline $\begin{array}{l}\text { In- and exclusion criteria are } \\
\text { adequately described (cases) }\end{array}$ & Inclusion and exclusion criteria are described. & yes \\
\hline $\begin{array}{l}\text { In- and exclusion criteria are } \\
\text { adequately described (controls) }\end{array}$ & NA & \\
\hline $\begin{array}{l}\text { Adequate participation by eligible } \\
\text { individuals ( } \geq 80 \%)\end{array}$ & The participation rate $74 \%$. & partial \\
\hline Study attrition & & $\mathrm{L}$ \\
\hline Adequate response rate $(\geq 80 \%)$ & The response rate is $99 \%$. & yes \\
\hline $\begin{array}{l}\text { Differences between participants } \\
\text { and non-participants }\end{array}$ & $\begin{array}{l}\text { There were no significant differences between participants } \\
\text { and non-participants concerning age }(p=0.62) \text { and } \\
\text { inclusion criteria for diagnosing peanut allergy }(p=0.17) \text {. } \\
\text { Single or multiple food allergies were not compared. }\end{array}$ & partial \\
\hline \multicolumn{2}{|c|}{ Diagnostic intervention measurement } & $\mathrm{L}$ \\
\hline $\begin{array}{l}\text { Clear definition of diagnostic } \\
\text { intervention }\end{array}$ & $\begin{array}{l}\text { A single dose peanut food challenge is performed in all } \\
\text { cases, according to a clear described protocol. Criteria for a } \\
\text { positive food challenge are reported. }\end{array}$ & yes \\
\hline $\begin{array}{l}\text { Methods the same for all study } \\
\text { participants }\end{array}$ & $\begin{array}{l}\text { Yes, the same recipe and food challenge protocol is used } \\
\text { for all participants. }\end{array}$ & yes \\
\hline $\begin{array}{l}\text { Diagnostic intervention not } \\
\text { performed in control population }\end{array}$ & NA & \\
\hline Outcome measurement & & $\mathrm{L}$ \\
\hline $\begin{array}{l}\text { Timing of quality of life } \\
\text { measurement }\end{array}$ & $\begin{array}{l}\text { The time point of the HRQL questionnaire before the OFC } \\
\text { was not described. The follow-up questionnaire is one } \\
\text { month after the OFC. }\end{array}$ & partial \\
\hline $\begin{array}{l}\text { Methods the same for all study } \\
\text { participants }\end{array}$ & $\begin{array}{l}\text { Questionnaires were self-administered by parents (FAQLQ- } \\
\text { PF 0-12 years) or children (FAQLQ-CF } 8-17 \text { years). It was } \\
\text { not described which parent should fill out the } \\
\text { questionnaire. }\end{array}$ & Yes \\
\hline \multicolumn{2}{|l|}{ Study confounding } & $\mathrm{M}$ \\
\hline All important confounders are & Yes: age. & partial \\
\hline measured & $\begin{array}{l}\text { No: number of food allergies, history of anaphylaxis and } \\
\text { baseline quality of life scores. }\end{array}$ & \\
\hline All important confounders are & Yes: age. & partial \\
\hline accounted for in the analyses & $\begin{array}{l}\text { No: number of food allergies, history of anaphylaxis and } \\
\text { baseline quality of life scores. }\end{array}$ & \\
\hline \multicolumn{2}{|l|}{$\begin{array}{l}\text { Statistical analysis and } \\
\text { reporting }\end{array}$} & $\mathrm{H}$ \\
\hline $\begin{array}{l}\text { Analysis conducted according to a } \\
\text { conceptual framework }\end{array}$ & $\begin{array}{l}\text { Statistical analysis is appropriate and based on a } \\
\text { conceptual framework. The methods for model building are } \\
\text { unclear. }\end{array}$ & partial \\
\hline Full outcome data are available & $\begin{array}{l}\text { No full outcome data are reported. Mean quality of life } \\
\text { scores before and after the diagnostic interventions or } \\
\text { mean differences are not reported, only an effect size. }\end{array}$ & no \\
\hline Overall risk of bias (publication) & Moderate & \\
\hline
\end{tabular}

Abbreviations: FAQLQ, Food Allergy Quality of Life Questionnaire (different versions: PF, parent form and $C F$, child form); $H$, high; L, low; M, moderate; NA, not applicable; OFC, oral food challenge. 
Supplemental Table 4. Risk of bias assessment (continued)

\begin{tabular}{|c|c|c|}
\hline \multicolumn{3}{|l|}{ van der Valk $2016^{26}$} \\
\hline Study participation & & $\mathrm{L}$ \\
\hline $\begin{array}{l}\text { Method used to identify } \\
\text { population (cases) }\end{array}$ & Consecutive enrollment. & yes \\
\hline $\begin{array}{l}\text { Method used to identify } \\
\text { population (controls) }\end{array}$ & NA & \\
\hline $\begin{array}{l}\text { In- and exclusion criteria are } \\
\text { adequately described (cases) }\end{array}$ & Inclusion and exclusion criteria are described. & yes \\
\hline $\begin{array}{l}\text { In- and exclusion criteria are } \\
\text { adequately described (controls) }\end{array}$ & NA & \\
\hline $\begin{array}{l}\text { Adequate participation by eligible } \\
\text { individuals }(\geq 80 \%)\end{array}$ & The participation rate of the initial study is around $40 \%$. & partial \\
\hline Study attrition & & M \\
\hline Adequate response rate ( $\geq 80 \%)$ & The response rate is $63 \%$. & partial \\
\hline $\begin{array}{l}\text { Differences between participants } \\
\text { and non-participants }\end{array}$ & $\begin{array}{l}\text { There was no significant difference in gender }(p=0.92) \text {, age } \\
(p=0.74) \text { and history of cashew nut allergy }(p=0.12) \text {, among } \\
\text { others. Differences in number of other food allergies were } \\
\text { not reported. }\end{array}$ & partial \\
\hline \multicolumn{2}{|c|}{ Diagnostic intervention measurement } & $\mathrm{L}$ \\
\hline $\begin{array}{l}\text { Clear definition of diagnostic } \\
\text { intervention }\end{array}$ & $\begin{array}{l}\text { A DBPCFC is performed in all cases, according to a clear } \\
\text { described protocol. }\end{array}$ & yes \\
\hline $\begin{array}{l}\text { Methods the same for all study } \\
\text { participants }\end{array}$ & $\begin{array}{l}\text { Yes, all study participants were challenged according to the } \\
\text { same DBPCFC protocol. }\end{array}$ & yes \\
\hline $\begin{array}{l}\text { Diagnostic intervention not } \\
\text { performed in control population }\end{array}$ & NA & \\
\hline Outcome measurement & & L \\
\hline $\begin{array}{l}\text { Timing of quality of life } \\
\text { measurement }\end{array}$ & $\begin{array}{l}\text { The time point of the HRQL questionnaire before the OFC } \\
\text { was not described. The follow-up questionnaire is six } \\
\text { months after the OFC. }\end{array}$ & partial \\
\hline $\begin{array}{l}\text { Methods the same for all study } \\
\text { participants }\end{array}$ & $\begin{array}{l}\text { Yes, age appropriate questionnaires were used in all } \\
\text { patients. No details on methods of administration or which } \\
\text { parent should fill out the questionnaire. }\end{array}$ & yes \\
\hline Study confounding & & $\mathrm{L}$ \\
\hline $\begin{array}{l}\text { All important confounders are } \\
\text { measured }\end{array}$ & $\begin{array}{l}\text { Yes: age, single or multiple food allergies and baseline } \\
\text { quality of life scores. } \\
\text { No: history of anaphylaxis. }\end{array}$ & partial \\
\hline $\begin{array}{l}\text { All important confounders are } \\
\text { accounted for in the analyses }\end{array}$ & $\begin{array}{l}\text { Yes: age, single or multiple food allergies and baseline } \\
\text { quality of life scores. } \\
\text { No: history of anaphylaxis. }\end{array}$ & yes \\
\hline $\begin{array}{l}\text { Statistical analysis and } \\
\text { reporting }\end{array}$ & & L \\
\hline $\begin{array}{l}\text { Analysis conducted according to a } \\
\text { conceptual framework }\end{array}$ & $\begin{array}{l}\text { Statistical analysis, including model building, is appropriate } \\
\text { and based on a conceptual framework. }\end{array}$ & yes \\
\hline Full outcome data are available & Full outcome data are reported. & yes \\
\hline
\end{tabular}

Overall risk of bias (publication) Low

Abbreviations: DBPCFC, double-blind placebo-controlled food challenge; HRQL, health-related quality of life; $H$, high; $L$, low; $M$, moderate; $N A$, not applicable; OFC, oral food challenge. 
Supplemental Table 4. Risk of bias assessment (continued)

\begin{tabular}{l}
\hline Franxman $\mathbf{2 0 1 5}^{28}$ \\
\hline Study participation \\
Method used to identify \\
population (cases)
\end{tabular}

Method used to identify population (controls) In- and exclusion criteria are adequately described (cases) In- and exclusion criteria are adequately described (controls) Adequate participation by eligible individuals ( $\geq 80 \%$ )

\section{Study attrition}

Adequate response rate ( $\geq 80 \%$ )

Differences between participants and non-participants

\section{Diagnostic intervention \\ measurement}

Clear definition of diagnostic intervention

Methods the same for all study participants

Diagnostic intervention not performed in control population

\section{Outcome measurement}

Timing of quality of life

measurement

Methods the same for all study participants

\section{Study confounding}

All important confounders are measured

All important confounders are accounted for in the analyses

\section{Statistical analysis and} reporting

Analysis conducted according to a conceptual framework

Full outcome data are available
No consecutive enrollment. Patients who had undergone an OFC were identified from a divisional database, with additional cases identified by review of International Classification of Disease coding, Current Procedural Terminology coding for an OFC, and chart review of the clinic schedules.

Sampling and recruitment of the control cohort is not described.

Inclusion criteria are not adequately described, while exclusion criteria are.

Inclusion and exclusion criteria of the control population are not described.

The participation rate is $34 \%$. For the control group, the participation rate is unknown.

The response rate for cases is less than $50 \%$. The response rate for controls is unknown.

Not described.

H

no

unsure

It is not described whether open or double blind challenges were performed and no food challenge protocol was provided.

Not described.

unsure

Yes, the control population did not undergo an OFC.

yes

L

Patients participated at a random time point $\leq 11$ years after an OFC (median duration in months given), or $\leq 11$ years after a food allergy diagnosis without an OFC.

Caregivers were contacted via mail, and via follow-up telephone calls. The surveys were administered to the control group within the same time frame as for the OFC group with the same contact methodology.

Yes: age, number of food allergies and history of anaphylaxis. No: baseline quality of life scores.

Yes: age, history of anaphylaxis. No: number of food allergies and baseline quality of life scores.

partial partial

yes

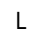

Statistical analysis, including model building, is appropriate and based on a conceptual framework.

Full outcome data are reported.

yes

yes

Overall risk of bias (publication) High

Abbreviations: $H$, high; $L$, low; $M$, moderate; OFC, oral food challenge. 
Supplemental Table 4. Risk of bias assessment (continued)

Soller $\mathbf{2 0 1 4} \mathbf{2}^{29}$
Method used to identify
population (cases)
Method used to identify
population (controls)
In- and exclusion criteria are
adequately described (cases)
In- and exclusion criteria are
adequately described (controls)
Adequate participation by eligible
individuals ( $\geq 80 \%$ )

\section{Study attrition}

Adequate response rate ( $\geq 80 \%$ )

Differences between participants and non-participants

\section{Diagnostic intervention}

\section{measurement}

Clear definition of diagnostic intervention

Methods the same for all study participants

Diagnostic intervention not performed in control population

\section{Outcome measurement}

Timing of quality of life measurement

Methods the same for all study participants

\section{Study confounding}

All important confounders are measured

All important confounders are accounted for in the analyses

\section{Statistical analysis and}

reporting

Analysis conducted according to a conceptual framework

Full outcome data are available
No consecutive enrollment. Participants were randomly selected from the food challenge waiting list.

NA

Inclusion and exclusion are not described.

no

NA

The response rate on questionnaires at all time-points is $92 \%$ of all eligible patients. Therefor also initial study participation can be considered adequate.

The response rate is $92 \%$.

yes

Not described.

unsure

L

partial

An open OFC is performed, without a clear described protocol. Criteria for a positive or negative food challenge are described.

All food challenges were open and performed in the same allergy center.

NA

yes

2 months before the OFC, on the day of the OFC, and 2 $\mathrm{L}$

yes months and 6 months after the OFC.

The methods and setting of outcome measurement (quality of life questionnaires administered) was not described.

Yes: age, baseline quality of life scores.

No: number of food allergies, history of anaphylaxis.

No (important) confounders were considered in the final analysis.

partial

$\mathrm{H}$

partial

no

M

Statistical analysis is appropriate and based on a

partial conceptual framework. The methods for model building are unclear.

No full outcome data are reported, only differences in

partial quality of life scores.

Overall risk of bias (publication) High

Abbreviations: $H$, high; $L$, low; $M$, moderate; NA, not applicable; OFC, oral food challenge. 
Supplemental Table 4. Risk of bias assessment (continued)

\begin{tabular}{|c|c|c|}
\hline \multicolumn{3}{|l|}{ Knibb 2012 $2^{33}$} \\
\hline Study participation & & M \\
\hline $\begin{array}{l}\text { Method used to identify } \\
\text { population (cases) }\end{array}$ & $\begin{array}{l}\text { No details described on the recruitment of the study } \\
\text { participants. }\end{array}$ & no \\
\hline $\begin{array}{l}\text { Method used to identify } \\
\text { population (controls) }\end{array}$ & $\begin{array}{l}\text { No details described on the recruitment of the study } \\
\text { participants. }\end{array}$ & no \\
\hline $\begin{array}{l}\text { In- and exclusion criteria are } \\
\text { adequately described (cases) }\end{array}$ & Inclusion and exclusion criteria are described. & yes \\
\hline $\begin{array}{l}\text { In- and exclusion criteria are } \\
\text { adequately described (controls) }\end{array}$ & Inclusion and exclusion criteria are described. & yes \\
\hline $\begin{array}{l}\text { Adequate participation by eligible } \\
\text { individuals }(\geq 80 \%)\end{array}$ & The participation rate of eligible patients is $59 \%$. & partial \\
\hline Study attrition & & $\mathrm{H}$ \\
\hline Adequate response rate ( $\geq 80 \%)$ & The response rate is $79 \%$. & partial \\
\hline $\begin{array}{l}\text { Differences between participants } \\
\text { and non-participants }\end{array}$ & Not described. & unsure \\
\hline $\begin{array}{l}\text { Diagnostic intervention } \\
\text { measurement }\end{array}$ & & M \\
\hline $\begin{array}{l}\text { Clear definition of diagnostic } \\
\text { intervention }\end{array}$ & $\begin{array}{l}\text { An open OFC was performed, without a clear described } \\
\text { protocol. No criteria for positive or negative OFCs were } \\
\text { described. }\end{array}$ & partial \\
\hline $\begin{array}{l}\text { Methods the same for all study } \\
\text { participants }\end{array}$ & $\begin{array}{l}\text { All challenges were open OFCs. No further details were } \\
\text { provided. }\end{array}$ & unsure \\
\hline $\begin{array}{l}\text { Diagnostic intervention not } \\
\text { performed in control population }\end{array}$ & $\begin{array}{l}\text { Yes, the control population did not undergo an oral food } \\
\text { chall. }\end{array}$ & yes \\
\hline Outcome measurement & & $\mathrm{L}$ \\
\hline $\begin{array}{l}\text { Timing of quality of life } \\
\text { measurement }\end{array}$ & $\begin{array}{l}\text { Baseline (following a clinic appointment) and follow-up (3-6 } \\
\text { months following OFC, or in the case of non-challenged } \\
\text { families, 3-6 months following the return of baseline } \\
\text { questionnaire) }\end{array}$ & yes \\
\hline $\begin{array}{l}\text { Methods the same for all study } \\
\text { participants }\end{array}$ & $\begin{array}{l}\text { Children and mothers completed baseline and 3-6 months } \\
\text { follow-up questionnaires at home. Questionnaires during } \\
\text { oral food challenge were completed in the clinical setting. }\end{array}$ & yes \\
\hline Study confounding & & M \\
\hline $\begin{array}{l}\text { All important confounders are } \\
\text { measured }\end{array}$ & Yes, all important confounder data are available at baseline. & Yes \\
\hline $\begin{array}{l}\text { All important confounders are } \\
\text { accounted for in the analyses }\end{array}$ & $\begin{array}{l}\text { No (important) confounders were considered in the final } \\
\text { analysis. }\end{array}$ & no \\
\hline $\begin{array}{l}\text { Statistical analysis and } \\
\text { reporting }\end{array}$ & & $\mathrm{L}$ \\
\hline $\begin{array}{l}\text { Analysis conducted according to a } \\
\text { conceptual framework }\end{array}$ & $\begin{array}{l}\text { Statistical analysis is appropriate and based on a } \\
\text { conceptual framework. No model is built. }\end{array}$ & yes \\
\hline Full outcome data are available & Full outcome data are reported. & yes \\
\hline Overall risk of bias (publication) & High & \\
\hline
\end{tabular}

Abbreviations: $H$, high; $L$, low; $M$, moderate; OFC, oral food challenge. 
Supplemental Table 4. Risk of bias assessment (continued)

van der Velde $\mathbf{2 0 1 2}^{32}$
Study participation
pethod used to identify

Method used to identify
population (controls)

population (controls)

In- and exclusion criteria are adequately described (cases)

In- and exclusion criteria are adequately described (controls)

Adequate participation by eligible individuals ( $\geq 80 \%$ )

\section{Study attrition}

Adequate response rate ( $\geq 80 \%$ )

Differences between participants

and non-participants

\section{Diagnostic intervention \\ measurement}

Clear definition of diagnostic intervention

Methods the same for all study participants

Diagnostic intervention not

performed in control population

\section{Outcome measurement}

Timing of quality of life

measurement

Methods the same for all study participants

\section{Study confounding}

All important confounders are measured

All important confounders are accounted for in the analyses

\section{Statistical analysis and reporting}

Analysis conducted according to a conceptual framework

Full outcome data are available
All patients visiting the clinic with suspected food allergy $\mathrm{L}$ yes were placed on the waiting list for a DBPCFC on a firstcome, first-served basis. Participants who were expected to have a DBPCFC within 6 months were included in the experimental group (cases).

Participants on the waiting list who were not expected to

partial have a DBPCFC within 6 months were included in the control group. Additionally, participants recruited through Dutch food allergy support organizations were included in the control group when they reported a physician-

diagnosed food allergy (skin prick test, sIgE). Methods for recruitment through the support organization are unclear. Inclusion and exclusion criteria are described.

Inclusion and exclusion criteria are described.

yes

The participation rate is $76 \%$.

partial

The response rate is $74 \%$.

Not described.

\section{$\mathrm{H}$ \\ partial}

unsure

A DBPCFC is performed in all cases according to a clear yes described protocol.

The two centers collaborate on uniform methodology of DBPCFC procedures.

Yes, the control population did not undergo an OFC.

Challenged cases: 1 month before (baseline) and 6 months after (follow-up) a DBPCFC. Unchallenged cases: twice with a 7-month interval without undergoing a DBPCFC.

All questionnaires were sent home and the same questionnaires were used in all study participants.

Yes, all important confounder data are available.

Yes: age, number of food allergies and baseline quality of life scores.

yes

No: history of anaphylaxis.

$$
\mathrm{L}
$$

yes and based on a conceptual framework.

Full outcome data are reported.

yes

Overall risk of bias (publication) Moderate

Abbreviations: DBPCFC, double-blind placebo-controlled food challenge; $H$, high; $L$, low; $M$, moderate; NA, not applicable; OFC, oral food challenge. 
Supplemental Table 4. Risk of bias assessment (continued)

\begin{tabular}{|c|c|c|}
\hline \multicolumn{3}{|l|}{ Dunn Galvin $2010^{37}$} \\
\hline Study participation & & $\mathrm{L}$ \\
\hline $\begin{array}{l}\text { Method used to identify } \\
\text { population (cases) }\end{array}$ & $\begin{array}{l}\text { No consecutive enrollment, but a random sample of } \\
\text { participants recruited during specialist pediatric allergy } \\
\text { clinic. No recruitment details are reported. }\end{array}$ & no \\
\hline $\begin{array}{l}\text { Method used to identify } \\
\text { population (controls) }\end{array}$ & NA & \\
\hline $\begin{array}{l}\text { In- and exclusion criteria are } \\
\text { adequately described (cases) }\end{array}$ & Inclusion and exclusion criteria are described. & yes \\
\hline $\begin{array}{l}\text { In- and exclusion criteria are } \\
\text { adequately described (controls) }\end{array}$ & NA & \\
\hline $\begin{array}{l}\text { Adequate participation by eligible } \\
\text { individuals }(\geq 80 \%)\end{array}$ & The participation rate is $94 \%$. & yes \\
\hline Study attrition & & $\mathrm{M}$ \\
\hline Adequate response rate $(\geq 80 \%)$ & The response rate is $96 \%$. & yes \\
\hline $\begin{array}{l}\text { Differences between participants } \\
\text { and non-participants }\end{array}$ & Not described. & unsure \\
\hline $\begin{array}{l}\text { Diagnostic intervention } \\
\text { measurement }\end{array}$ & & $\mathrm{H}$ \\
\hline $\begin{array}{l}\text { Clear definition of diagnostic } \\
\text { intervention }\end{array}$ & Not described. & no \\
\hline $\begin{array}{l}\text { Methods the same for all study } \\
\text { participants }\end{array}$ & Not described. & no \\
\hline $\begin{array}{l}\text { Diagnostic intervention not } \\
\text { performed in control population }\end{array}$ & NA & \\
\hline Outcome measurement & & $\mathrm{L}$ \\
\hline $\begin{array}{l}\text { Timing of quality of life } \\
\text { measurement }\end{array}$ & $\begin{array}{l}\text { Before the OFC (morning of the OFC) and at } 2 \text { and } 6 \\
\text { months after the OFC. }\end{array}$ & yes \\
\hline $\begin{array}{l}\text { Methods the same for all study } \\
\text { participants }\end{array}$ & $\begin{array}{l}\text { Mothers were asked to fill-out the questionnaire in the } \\
\text { Children's Day Unit on the morning of the OFC. } \\
\text { Questionnaires were again completed at home } 2 \text { months } \\
\text { and } 6 \text { months after the OFC. }\end{array}$ & yes \\
\hline Study confounding & & M \\
\hline $\begin{array}{l}\text { All important confounders are } \\
\text { measured }\end{array}$ & $\begin{array}{l}\text { Yes: age, number of food allergies, history of anaphylaxis } \\
\text { and baseline quality of life scores. }\end{array}$ & yes \\
\hline $\begin{array}{l}\text { All important confounders are } \\
\text { accounted for in the analyses }\end{array}$ & $\begin{array}{l}\text { No (important) confounders were considered in the final } \\
\text { analysis. }\end{array}$ & no \\
\hline $\begin{array}{l}\text { Statistical analysis and } \\
\text { reporting }\end{array}$ & & L \\
\hline $\begin{array}{l}\text { Analysis conducted according to a } \\
\text { conceptual framework }\end{array}$ & $\begin{array}{l}\text { It is unclear what variables were taken into account in the } \\
\text { final analysis. }\end{array}$ & partial \\
\hline Full outcome data are available & Full outcome data are reported. & yes \\
\hline Overall risk of bias (publication) & Moderate & \\
\hline
\end{tabular}

Abbreviations: $H$, high; $L$, low; $M$, moderate; NA, not applicable; OFC, oral food challenge. 
Supplemental Table 5a. Sensitivity analysis correlation coefficient (studies including patients with suspected food allergy)

\begin{tabular}{|c|c|c|c|c|c|c|c|c|c|}
\hline \multirow{3}{*}{$\begin{array}{l}\text { DunnGalvin } \\
\text { FAQLQ-PF (0-2M) }\end{array}$} & \multicolumn{3}{|c|}{$r=0.5$} & \multicolumn{3}{|c|}{$r=0.2$} & \multicolumn{3}{|c|}{$r=0.8$} \\
\hline & \multirow[t]{2}{*}{$\mathrm{MD}$} & \multicolumn{2}{|c|}{$95 \% \mathrm{CI}$} & \multirow[t]{2}{*}{$\mathrm{MD}$} & \multicolumn{2}{|c|}{$95 \% \mathrm{CI}$} & \multirow[t]{2}{*}{$\mathrm{MD}$} & \multicolumn{2}{|c|}{$95 \% \mathrm{CI}$} \\
\hline & & & & & & & & & \\
\hline Total & $0.60^{*}$ & 0.28 & 0.91 & $0.60^{*}$ & 0.20 & 0.99 & $0.60^{*}$ & 0.40 & 0.8 \\
\hline Positive patients & $0.50^{*}$ & 0.08 & 0.92 & 0.50 & -0.04 & 1.04 & $.50^{\star}$ & 0.23 & 0.77 \\
\hline Negative patients & $0.70^{*}$ & 0.24 & 1.16 & $0.70^{*}$ & 0.11 & 1.29 & $0.70^{*}$ & 0.41 & 0.99 \\
\hline All patients (EI) & $0.75^{*}$ & 0.46 & 1.05 & $0.75^{*}$ & 0.38 & 1.13 & $0.75^{\star *}$ & 0.57 & 0.94 \\
\hline All patients (FA) & $0.64^{*}$ & 0.33 & 0.95 & $0.64^{*}$ & 0.25 & 1.04 & $0.64^{*}$ & 0.45 & 0.84 \\
\hline All patients (SDI) & $0.69 *$ & 0.40 & 0.98 & $0.69 *$ & 0.33 & 1.06 & $0.69 * \star$ & 0.50 & 0.88 \\
\hline Positive patients (EI) & $0.90^{* *}$ & 0.51 & 1.29 & $0.90^{*}$ & 0.4 & 1.4 & $0.90^{\star *}$ & 0.65 & 1.15 \\
\hline Positive patients & 0.40 & -0.06 & 0.86 & 0.40 & -0.18 & 0.98 & $0.40^{*}$ & 0.11 & 0.69 \\
\hline Positive patients (SDI) & 0.40 & -0.04 & 0.84 & 0.40 & -0.14 & 0.94 & $.40^{*}$ & 0.11 & 0.69 \\
\hline Negative patien & $0.60^{*}$ & 0.15 & 1.05 & $0.60^{*}$ & 0.03 & 1.17 & $0.60^{*}$ & 0.31 & 0.89 \\
\hline Negat & $0.90 *$ & 0.48 & 1.32 & $0.90 *$ & 0.37 & 1.43 & 0.9 & 0.63 & 1.17 \\
\hline Negative patients (S & $1.00^{* *}$ & 0.62 & 1.38 & $1.00 * *$ & 0.52 & 1.48 & 1.00 ** & 0.76 & 1.24 \\
\hline \multicolumn{10}{|l|}{ FAQLQ-PF (2-6M) } \\
\hline Total & $1.14^{\star \star}$ & 0.82 & 1.46 & $1.14^{\star \star}$ & 0.74 & 1.54 & $1.14^{\star \star}$ & 0.94 & 1.34 \\
\hline Positive patients & $0.70^{*}$ & 0.26 & 1.14 & $0.70 *$ & 0.14 & 1.26 & $0.70 *$ & 0.42 & 0.98 \\
\hline Neg & 1.60 ** & 1.14 & 2.06 & $1.60 * *$ & 1.01 & 2.19 & 1.60 ** & 1.31 & 1.89 \\
\hline All $p$ & $1.19^{* *}$ & 0.89 & 1.419 & $1.19 * *$ & 0.81 & 1.57 & 1.1 & 1.00 & 1.38 \\
\hline All $p$ & $0.99 * *$ & 0.67 & 1.31 & $0.99 * *$ & 0.58 & 1.40 & 0.99 & 0.78 & 1.19 \\
\hline All $p$ & $1.14^{* *}$ & 0.87 & 1.41 & $1.14^{\star *}$ & 0.79 & 1.48 & ** & 0.96 & 1.31 \\
\hline & & 0.42 & 8 & $0.80 *$ & 0.32 & 1.28 & & 0.56 & 1.04 \\
\hline Posi & * & 0.02 & 0.98 & .50 & -0.11 & 1.11 & * & 0.19 & 0.81 \\
\hline & $60^{*}$ & 0.22 & 0.98 & $60 *$ & 0.12 & 08 & $60^{*}$ & 0.36 & 0.84 \\
\hline Nega & $1.60^{\star *}$ & 1.18 & 2.02 & $1.60^{\star \star}$ & 1.07 & 2.13 & $60^{\star \star}$ & 1.33 & 1.87 \\
\hline Nega & $1.50 * *$ & 1.03 & 1.92 & $1.50^{\star \star \star}$ & 0.97 & 2.03 & $1.50^{\star *}$ & 1.23 & 1.77 \\
\hline Negative & $1.70 * *$ & 1.33 & 2.07 & 1.70 ** & 1.23 & 2.17 & $1.70^{* *}$ & 1.46 & 1.94 \\
\hline \multirow[t]{2}{*}{ Soller } & \multicolumn{3}{|c|}{$r=0.5$} & \multicolumn{3}{|c|}{$r=0.2$} & \multicolumn{3}{|c|}{$r=0.8$} \\
\hline & MD & \multicolumn{2}{|c|}{$95 \% \mathrm{CI}$} & MD & \multicolumn{2}{|c|}{$95 \% \mathrm{CI}$} & MD & \multicolumn{2}{|c|}{$95 \% \mathrm{CI}$} \\
\hline \multicolumn{10}{|l|}{ FAQLQ-PF (0-2M) } \\
\hline Total & $0.86^{\star *}$ & 0.719 & 1.003 & $0.86^{\star \star}$ & 0.68 & 1.04 & $0.86^{\star \star}$ & 0.77 & 0.95 \\
\hline All patients (EI) & $0.60^{\star}$ & 0.48 & 0.72 & $0.60^{\star}$ & 0.46 & 0.75 & $0.60^{\star \star}$ & 0.51 & 0.70 \\
\hline All patients (FA) & $0.95^{\star *}$ & 0.74 & 1.16 & $0.95^{* *}$ & 0.68 & 1.22 & $0.95^{\star *}$ & 0.82 & 1.08 \\
\hline All patients (SDI) & $1.05^{\star *}$ & 0.91 & 1.19 & $1.05^{\star *}$ & 0.87 & 1.23 & $1.05^{* *}$ & 0.96 & 1.14 \\
\hline \multicolumn{10}{|l|}{ FAQLQ-PF (2-6M) } \\
\hline Total & & -0.01 & 0.99 & 0.49 & -0.14 & 1.17 & $0.49^{*}$ & 0.178 & 0.81 \\
\hline & & 0.08 & 0.92 & 0.50 & -0.04 & 1.04 & $0.50^{*}$ & 0.23 & 0.77 \\
\hline & $0.70^{*}$ & 0.12 & 1.28 & 0.70 & -0.02 & 1.42 & $0.70^{*}$ & 0.32 & 1.08 \\
\hline All patients (SDI) & $0.55^{*}$ & 0.02 & 1.08 & 0.55 & -0.12 & 1.22 & $0.55^{*}$ & 0.21 & 0.89 \\
\hline
\end{tabular}

* significant change in $\mathrm{HRQL}$

** significant and clinically relevant change in $\mathrm{HRQL}$

Abbreviations: $\mathrm{Cl}$, confidence interval; FAQLQ-PF, Food Allergy Quality of Life Questionnaire Parent Form (different domains: El, Emotional Impact; FA, Food Anxiety; SDI, Social and Dietary Impact); M, months; $M D$, mean difference; $r$, correlation coefficient. 
Supplemental Table 5a. Sensitivity analysis correlation coefficient (studies including patients with suspected food allergy) (continued)

\begin{tabular}{|c|c|c|c|c|c|c|c|c|c|}
\hline \multirow[t]{2}{*}{ van der Valk } & \multicolumn{3}{|c|}{$r=0.5$} & \multicolumn{3}{|c|}{$r=0.2$} & \multicolumn{3}{|c|}{$r=0.8$} \\
\hline & \multirow[t]{2}{*}{$\mathrm{MD}$} & \multicolumn{2}{|c|}{$95 \% \mathrm{CI}$} & \multirow[t]{2}{*}{$\mathrm{MD}$} & \multicolumn{2}{|c|}{$95 \% \mathrm{CI}$} & \multirow[t]{2}{*}{$\mathrm{MD}$} & \multicolumn{2}{|c|}{$95 \% \mathrm{CI}$} \\
\hline \multicolumn{7}{|l|}{ FAQLQ-PF (0-6M) } & & & \\
\hline Total & -0.06 & -0.32 & 0.2 & -0.06 & -0.38 & 0.26 & -0.06 & -0.22 & 0.1 \\
\hline Positive patients & -0.11 & -0.41 & 0.19 & -0.11 & -0.48 & 0.26 & -0.11 & -0.31 & 0.09 \\
\hline Negative patients & 0.1 & -0.4 & 0.6 & 0.1 & -0.52 & 0.72 & 0.1 & -0.24 & 0.44 \\
\hline All patients (EI) & -0.09 & -0.33 & 0.15 & -0.09 & -0.39 & 0.21 & -0.09 & -0.24 & 0.06 \\
\hline All patients (FA) & -0.01 & -0.27 & 0.25 & -0.01 & -0.34 & 0.32 & -0.01 & -0.18 & 0.16 \\
\hline All patients (SDI) & -0.07 & -0.31 & 0.17 & -0.07 & -0.37 & 0.23 & -0.07 & -0.22 & 0.08 \\
\hline Positive patients (EI) & -0.16 & -0.57 & 0.25 & -0.16 & -0.59 & 0.27 & -0.16 & -0.54 & 0.22 \\
\hline Positive patients & -0.08 & -0.37 & & -0.08 & -0.39 & & & -0.34 & 0.18 \\
\hline Positive patien & -0.08 & -0.37 & 0.21 & -0.08 & -0.39 & 0.23 & -0.08 & -0.34 & 0.18 \\
\hline Negat & & -0.76 & & 0.11 & -0.79 & & 0.11 & -0.71 & 0.93 \\
\hline Negat & & -0.74 & & 0.19 & -0.77 & 15 & 0.19 & -0.69 & 1.07 \\
\hline Negative patients (S & -0.03 & -1.04 & & -0.03 & -1.07 & 1.01 & -0.03 & -0.99 & 0.93 \\
\hline \multicolumn{10}{|l|}{ FAQLQ-TF (0-6M) } \\
\hline Total & & -0.18 & & & -0.3 & 0.86 & 0.28 & -0.02 & 0.58 \\
\hline Posit & -0.06 & -0.55 & 0.43 & -0.06 & -0.67 & 0.55 & -0.06 & -0.37 & 0.25 \\
\hline Nega & $1.18^{*}$ & 0.18 & & 1.18 & -0.06 & & & & 1.87 \\
\hline All pa & & -0.25 & & 0 & -0.37 & & .21 & -0.09 & 0.51 \\
\hline & & -0.29 & & & -0.41 & & & -0.13 & 0.47 \\
\hline & $0.47^{\star}$ & 0.01 & & 47 & -0.11 & 1.0 & $7^{*}$ & 0.17 & 0.77 \\
\hline & & & & & -0.18 & 1.04 & $0.43^{*}$ & 0.12 & 0.74 \\
\hline Posit & -0.15 & -0.64 & & -0.15 & -0.76 & 0.46 & -0.15 & -0.46 & 0.16 \\
\hline & 0.26 & -0.23 & 0.75 & 0.26 & -0.35 & 0.87 & 0.26 & -0.05 & 0.57 \\
\hline & 0.9 & -0.1 & & 0.9 & -0.34 & 2.14 & $0.9^{*}$ & 0.21 & 1.59 \\
\hline Negati & $1.49 *$ & 0.49 & & $1.49 *$ & 0.25 & & $1.49 * \star$ & 0.8 & 2.18 \\
\hline Negative patients $R$ & & & & & & & & & 2.00 \\
\hline \multicolumn{10}{|l|}{ FAQLQ-CF (0-6M) } \\
\hline Total & -0.17 & -0.65 & 0.31 & -0.17 & -0.78 & 0.44 & -0.17 & -0.48 & 0.14 \\
\hline Positive pat & -0.19 & -0.74 & 0.36 & -0.19 & -0.88 & 0.5 & -0.19 & -0.54 & 0.16 \\
\hline Negative patients & -0.1 & -1.08 & 0.88 & -0.1 & -1.34 & 1.14 & -0.1 & -0.72 & 0.52 \\
\hline All patients (AA) & -0.51 & -0.99 & -0.03 & -0.51 & -1.12 & 0.1 & -0.51 & -0.82 & -0.2 \\
\hline & -0.29 & -0.77 & & -0.29 & -0.9 & 0.32 & -0.29 & -0.6 & 0.02 \\
\hline & 0.18 & -0.3 & & & -0.43 & 0.79 & 0.18 & -0.13 & 0.49 \\
\hline All pa & & -0.47 & & & -0.6 & 0.62 & & -0.3 & 0.32 \\
\hline & -0.53 & -1.08 & & & -1.22 & & & -0.88 & -0.18 \\
\hline Positive patients (RAE) & -0.33 & -0.88 & 0.22 & -0.33 & -1.02 & 0.36 & -0.33 & -0.68 & 0.02 \\
\hline Positive patients (EI) & -0.14 & -0.69 & 0.41 & -0.14 & -0.83 & 0.55 & -0.14 & -0.49 & 0.21 \\
\hline Positive patients(DR) & 0.27 & -0.28 & 0.82 & 0.27 & -0.42 & 0.96 & 0.27 & -0.08 & 0.62 \\
\hline Negative patients (AA) & -0.44 & -1.42 & 0.54 & -0.44 & -1.68 & 0.8 & -0.44 & -1.06 & 0.18 \\
\hline Negative patients RAE) & -0.18 & -1.16 & 0.8 & -0.18 & -1.42 & 1.06 & -0.18 & -0.8 & 0.44 \\
\hline & 0.41 & -0.57 & 1.39 & 0.41 & -0.83 & 1.65 & 0.41 & -0.21 & 1.03 \\
\hline Negative patients (DR) & -0.56 & -1.54 & 0.42 & -0.56 & -1.8 & 0.68 & -0.56 & -1.18 & 0.06 \\
\hline
\end{tabular}

${ }^{*}$ significant change in $\mathrm{HRQL}$

** significant and clinically relevant change in HRQL

Abbreviations: see next page. 
Supplemental Table 5a. Sensitivity analysis correlation coefficient (studies including patients with suspected food allergy) (continued)

\begin{tabular}{|c|c|c|c|c|c|c|c|c|c|}
\hline \multirow{3}{*}{$\begin{array}{l}\text { Van der Velde } \\
\text { FAQLQ-CF (0-6M) }\end{array}$} & \multicolumn{3}{|c|}{$r=0.5$} & \multicolumn{3}{|c|}{$r=0.2$} & \multicolumn{3}{|c|}{$r=0.8$} \\
\hline & \multirow[t]{2}{*}{ MD } & \multicolumn{2}{|c|}{$95 \%$ CI } & \multirow[t]{2}{*}{ MD } & \multicolumn{2}{|c|}{$95 \%$ CI } & \multirow[t]{2}{*}{ MD } & \multicolumn{2}{|c|}{$95 \% \mathrm{CI}$} \\
\hline & & & & & & & & & \\
\hline Total (with OFC) & $0.55^{*}$ & 0.18 & 0.92 & $0.55^{\star}$ & 0.08 & 1.02 & $0.55^{\star}$ & 0.32 & 0.78 \\
\hline Positive (with OFC) & 0.39 & -0.09 & 0.87 & 0.39 & -0.22 & 1.00 & $0.39^{*}$ & 0.08 & 0.70 \\
\hline Negative (with OFC) & $0.79^{*}$ & 0.17 & 1.41 & $0.79^{*}$ & 0.01 & 1.57 & $0.79^{*}$ & 0.40 & 1.18 \\
\hline Inconclusive (with OFC) & 0.24 & -1.64 & 2.12 & 0.24 & -2.14 & 2.62 & 0.24 & -0.95 & 1.43 \\
\hline Total (without OFC) & -0.07 & -0.61 & 0.47 & -0.07 & -0.76 & 0.62 & -0.07 & -0.41 & 0.27 \\
\hline $\begin{array}{l}\text { Total (with vs. without } \\
\text { OFC) }\end{array}$ & 0.62 & -2.26 & 3.50 & 0.62 & -2.26 & 3.50 & 0.62 & -2.26 & 3.50 \\
\hline FAQLQ-TF (0-6M) & & & & & & & & & \\
\hline Total (with OFC) & 0.24 & -0.11 & 0.59 & 0.24 & -0.19 & 0.67 & $0.24^{*}$ & 0.01 & 0.47 \\
\hline Positive (with OFC) & 0.01 & -0.40 & 0.42 & 0.01 & -0.51 & 0.53 & 0.01 & -0.25 & 0.27 \\
\hline Negative (with OFC) & $0.97^{*}$ & 0.35 & 1.59 & $0.97^{*}$ & 0.20 & 1.74 & $0.97^{* *}$ & 0.54 & 1.40 \\
\hline Inconclusive (with OFC) & -0.4 & -1.28 & 0.48 & -0.40 & -1.49 & 0.69 & -0.40 & -1.01 & 0.21 \\
\hline Total (without OFC) & 0.34 & -0.15 & 0.83 & 0.34 & -0.27 & 0.95 & $0.34^{*}$ & 0.03 & 0.65 \\
\hline $\begin{array}{l}\text { Total (with vs. without } \\
\text { OFC) }\end{array}$ & -0.1 & -2.84 & 2.64 & -0.10 & -2.87 & 2.67 & -0.10 & -2.74 & 2.54 \\
\hline FAQLQ-AF (0-6M) & & & & & & & & & \\
\hline Total (with OFC) & $0.54^{*}$ & 0.20 & 0.88 & $0.54^{*}$ & 0.11 & 0.97 & $0.54^{*}$ & 0.32 & 0.76 \\
\hline Positive (with OFC) & 0.35 & -0.09 & 0.79 & 0.35 & -0.20 & 0.90 & $0.35^{*}$ & 0.06 & 0.64 \\
\hline Negative (with OFC) & $0.88^{*}$ & 0.31 & 1.45 & $0.88^{*}$ & 0.16 & 1.60 & $0.88^{* *}$ & 0.52 & 1.24 \\
\hline Inconclusive (with OFC) & -0.23 & -1.03 & 0.57 & -0.23 & -1.23 & 0.77 & -0.23 & -0.76 & 0.30 \\
\hline Total (without OFC) & 0.11 & -0.56 & 0.78 & 0.11 & -0.74 & 0.96 & 0.11 & -0.31 & 0.53 \\
\hline $\begin{array}{l}\text { Total (with vs. without } \\
\text { OFC) }\end{array}$ & 0.43 & -3.43 & 4.29 & 0.43 & -3.44 & 4.30 & 0.43 & -3.36 & 4.22 \\
\hline
\end{tabular}

* significant change in HRQL

** significant and clinically relevant change in HRQL

Abbreviations: Cl, confidence interval; FAQLQ, Food Allergy Quality of Life Questionnaire (different versions: AF, Adult Form; CF, Child Form; TF, Teen Form; PF, Parent Form and different domains: AA, Allergen Avoidance; DR, Dietary Restrictions; El, Emotional Impact; FA, Food Anxiety; RAE, Risk of Accidental Exposure; SDI, Social and Dietary Impact); M, months; MD, mean difference; OFC, oral food challenge; $r$, correlation coefficient. 
Supplemental Table 5a. Sensitivity analysis correlation coefficient (studies including patients with suspected food allergy) (continued)

\begin{tabular}{|c|c|c|c|c|c|c|c|c|c|}
\hline \multirow[t]{2}{*}{ van der Valk } & \multicolumn{3}{|c|}{$r=0.5$} & \multicolumn{3}{|c|}{$r=0.2$} & \multicolumn{3}{|c|}{$r=0.8$} \\
\hline & \multirow[t]{2}{*}{ MD } & \multicolumn{2}{|c|}{$95 \% \mathrm{CI}$} & \multirow[t]{2}{*}{$\mathrm{MD}$} & \multicolumn{2}{|c|}{$95 \% \mathrm{CI}$} & \multirow[t]{2}{*}{ MD } & \multicolumn{2}{|c|}{$95 \% \mathrm{CI}$} \\
\hline \multicolumn{7}{|l|}{ FAQLQ-PF (0-6M) } & & & \\
\hline Total & -0.06 & -0.32 & 0.2 & -0.06 & -0.38 & 0.26 & -0.06 & -0.22 & 0.1 \\
\hline Positive patients & -0.11 & -0.41 & 0.19 & -0.11 & -0.48 & 0.26 & -0.11 & -0.31 & 0.09 \\
\hline Negative patients & 0.1 & -0.4 & 0.6 & 0.1 & -0.52 & 0.72 & 0.1 & -0.24 & 0.44 \\
\hline All patients (EI) & -0.09 & -0.33 & 0.15 & -0.09 & -0.39 & 0.21 & -0.09 & -0.24 & 0.06 \\
\hline All patients (FA) & -0.01 & -0.27 & 0.25 & -0.01 & -0.34 & 0.32 & -0.01 & -0.18 & 0.16 \\
\hline All patients (SDI) & -0.07 & -0.31 & 0.17 & -0.07 & -0.37 & 0.23 & -0.07 & -0.22 & 0.08 \\
\hline Positive patients (EI) & -0.16 & -0.57 & 0.25 & -0.16 & -0.59 & 0.27 & -0.16 & -0.54 & 0.22 \\
\hline Positive patients & -0.08 & -0.37 & & & & & & -0.34 & 0.18 \\
\hline Positive patients & -0.08 & -0.37 & & -0.08 & -0.39 & 0.23 & -0.08 & 0.34 & 0.18 \\
\hline Negati & 0.11 & -0.76 & & & -0.79 & & & -0.71 & 0.93 \\
\hline Negat & & -0.74 & & 0.19 & -0.77 & 15 & 0.19 & -0.69 & .07 \\
\hline Negative patients (S & -0.03 & -1.04 & & -0.03 & -1.07 & 1.01 & -0.03 & -0.99 & 0.93 \\
\hline \multicolumn{10}{|l|}{ FAQLQ-TF (0-6M) } \\
\hline Total & & -0.18 & & & -0.3 & 0.86 & 0.28 & -0.02 & 0.58 \\
\hline Posit & -0.06 & -0.55 & 0.43 & -0.06 & -0.67 & 0.55 & -0.06 & -0.37 & 0.25 \\
\hline Nega & $1.18^{*}$ & 0.18 & & 1.18 & -0.06 & & & 0.49 & 1.87 \\
\hline All pa & & -0.25 & & & -0.37 & & & -0.09 & 51 \\
\hline & & -0.29 & & & -0.41 & & & -0.13 & 0.47 \\
\hline & $0.47^{\star}$ & 0.01 & & 47 & -0.11 & 1 & $7^{*}$ & 0.17 & 0.77 \\
\hline & 0.43 & -0.06 & & & -0.18 & 1.04 & $0.43^{*}$ & 0.12 & 0.74 \\
\hline Positi & -0.15 & -0.64 & & -0.15 & -0.76 & 0.46 & -0.15 & -0.46 & 0.16 \\
\hline & 0.26 & -0.23 & 0.75 & 0.26 & -0.35 & 0.87 & 0.26 & -0.05 & 0.57 \\
\hline & 0.9 & -0.1 & & 0.9 & -0.34 & 2.14 & $0.9^{*}$ & 0.21 & 1.59 \\
\hline Negati & $1.49 *$ & 0.49 & & $1.49 *$ & 0.25 & 2.73 & $1.49 * \star$ & 0.8 & 2.18 \\
\hline Negative patients $R$ & & & & & & & & 0.62 & 2.00 \\
\hline \multicolumn{10}{|l|}{ FAQLQ-CF (0-6M) } \\
\hline Total & -0.17 & -0.65 & 0.31 & -0.17 & -0.78 & 0.44 & -0.17 & -0.48 & 0.14 \\
\hline Positive patients & -0.19 & -0.74 & 0.36 & -0.19 & -0.88 & 0.5 & -0.19 & -0.54 & 0.16 \\
\hline Negative patients & -0.1 & -1.08 & 0.88 & -0.1 & -1.34 & 1.14 & -0.1 & -0.72 & 0.52 \\
\hline All patients (AA) & -0.51 & -0.99 & -0.03 & -0.51 & -1.12 & 0.1 & -0.51 & -0.82 & -0.2 \\
\hline & -0.29 & -0.77 & & -0.29 & -0.9 & 0.32 & -0.29 & -0.6 & 0.02 \\
\hline & 0.18 & -0.3 & & & -0.43 & 0.79 & 0.18 & -0.13 & 0.49 \\
\hline All pa & & -0.47 & & & -0.6 & 0.62 & & -0.3 & 0.32 \\
\hline & -0.53 & -1.08 & & & -1.22 & & & -0.88 & -0.18 \\
\hline Positive patients (RAE) & -0.33 & -0.88 & 0.22 & -0.33 & -1.02 & 0.36 & -0.33 & -0.68 & 0.02 \\
\hline Positive patients (EI) & -0.14 & -0.69 & 0.41 & -0.14 & -0.83 & 0.55 & -0.14 & -0.49 & 0.21 \\
\hline Positive patients(DR) & 0.27 & -0.28 & 0.82 & 0.27 & -0.42 & 0.96 & 0.27 & -0.08 & 0.62 \\
\hline Negative patients (AA) & -0.44 & -1.42 & 0.54 & -0.44 & -1.68 & 0.8 & -0.44 & -1.06 & 0.18 \\
\hline Negative patients RAE) & -0.18 & -1.16 & 0.8 & -0.18 & -1.42 & 1.06 & -0.18 & -0.8 & 0.44 \\
\hline & 0.41 & -0.57 & 1.39 & 0.41 & -0.83 & 1.65 & 0.41 & -0.21 & 1.03 \\
\hline Negative patients (DR) & -0.56 & -1.54 & 0.42 & -0.56 & -1.8 & 0.68 & -0.56 & -1.18 & 0.06 \\
\hline
\end{tabular}

${ }^{*}$ significant change in $\mathrm{HRQL}$

** significant and clinically relevant change in HRQL

Abbreviations: see next page. 
Supplemental Table 5a. Sensitivity analysis correlation coefficient (studies including patients with suspected food allergy) (continued)

\begin{tabular}{|c|c|c|c|c|c|c|c|c|c|}
\hline \multirow{3}{*}{$\begin{array}{l}\text { Van der Velde } \\
\text { FAQLQ-CF (0-6M) }\end{array}$} & \multicolumn{3}{|c|}{$r=0.5$} & \multicolumn{3}{|c|}{$r=0.2$} & \multicolumn{3}{|c|}{$r=0.8$} \\
\hline & \multirow[t]{2}{*}{$\mathrm{MD}$} & \multicolumn{2}{|c|}{$95 \% \mathrm{CI}$} & \multirow[t]{2}{*}{$\mathrm{MD}$} & \multicolumn{2}{|c|}{$95 \% \mathrm{CI}$} & \multirow[t]{2}{*}{$\mathrm{MD}$} & \multicolumn{2}{|c|}{$95 \%$ CI } \\
\hline & & & & & & & & & \\
\hline Total (with OFC) & $0.55^{*}$ & 0.18 & 0.92 & $0.55^{\star}$ & 0.08 & 1.02 & $0.55^{\star}$ & 0.32 & 0.78 \\
\hline Positive (with OFC) & 0.39 & -0.09 & 0.87 & 0.39 & -0.22 & 1.00 & $0.39^{*}$ & 0.08 & 0.70 \\
\hline Negative (with OFC) & $0.79^{*}$ & 0.17 & 1.41 & $0.79^{*}$ & 0.01 & 1.57 & $0.79^{*}$ & 0.40 & 1.18 \\
\hline Inconclusive (with OFC) & 0.24 & -1.64 & 2.12 & 0.24 & -2.14 & 2.62 & 0.24 & -0.95 & 1.43 \\
\hline Total (without OFC) & -0.07 & -0.61 & 0.47 & -0.07 & -0.76 & 0.62 & -0.07 & -0.41 & 0.27 \\
\hline $\begin{array}{l}\text { Total (with vs. without } \\
\text { OFC) }\end{array}$ & 0.62 & -2.26 & 3.50 & 0.62 & -2.26 & 3.50 & 0.62 & -2.26 & 3.50 \\
\hline FAQLQ-TF (0-6M) & & & & & & & & & \\
\hline Total (with OFC) & 0.24 & -0.11 & 0.59 & 0.24 & -0.19 & 0.67 & $0.24^{*}$ & 0.01 & 0.47 \\
\hline Positive (with OFC) & 0.01 & -0.40 & 0.42 & 0.01 & -0.51 & 0.53 & 0.01 & -0.25 & 0.27 \\
\hline Negative (with OFC) & $0.97^{*}$ & 0.35 & 1.59 & $0.97^{*}$ & 0.20 & 1.74 & $0.97^{* *}$ & 0.54 & 1.40 \\
\hline Inconclusive (with OFC) & -0.4 & -1.28 & 0.48 & -0.40 & -1.49 & 0.69 & -0.40 & -1.01 & 0.21 \\
\hline Total (without OFC) & 0.34 & -0.15 & 0.83 & 0.34 & -0.27 & 0.95 & $0.34^{*}$ & 0.03 & 0.65 \\
\hline $\begin{array}{l}\text { Total (with vs. without } \\
\text { OFC) }\end{array}$ & -0.1 & -2.84 & 2.64 & -0.10 & -2.87 & 2.67 & -0.10 & -2.74 & 2.54 \\
\hline FAQLQ-AF (0-6M) & & & & & & & & & \\
\hline Total (with OFC) & $0.54^{*}$ & 0.20 & 0.88 & $0.54^{*}$ & 0.11 & 0.97 & $0.54^{*}$ & 0.32 & 0.76 \\
\hline Positive (with OFC) & 0.35 & -0.09 & 0.79 & 0.35 & -0.20 & 0.90 & $0.35^{*}$ & 0.06 & 0.64 \\
\hline Negative (with OFC) & $0.88^{*}$ & 0.31 & 1.45 & $0.88^{*}$ & 0.16 & 1.60 & $0.88^{* *}$ & 0.52 & 1.24 \\
\hline Inconclusive (with OFC) & -0.23 & -1.03 & 0.57 & -0.23 & -1.23 & 0.77 & -0.23 & -0.76 & 0.30 \\
\hline Total (without OFC) & 0.11 & -0.56 & 0.78 & 0.11 & -0.74 & 0.96 & 0.11 & -0.31 & 0.53 \\
\hline $\begin{array}{l}\text { Total (with vs. without } \\
\text { OFC) }\end{array}$ & 0.43 & -3.43 & 4.29 & 0.43 & -3.44 & 4.30 & 0.43 & -3.36 & 4.22 \\
\hline
\end{tabular}

* significant change in HRQL

** significant and clinically relevant change in HRQL

Abbreviations: Cl, confidence interval; FAQLQ, Food Allergy Quality of Life Questionnaire (different versions: AF, Adult Form; CF, Child Form; TF, Teen Form; PF, Parent Form and different domains: AA, Allergen Avoidance; DR, Dietary Restrictions; El, Emotional Impact; FA, Food Anxiety; RAE, Risk of Accidental Exposure; SDI, Social and Dietary Impact); M, months; MD, mean difference; OFC, oral food challenge; $r$, correlation coefficient. 
Supplemental Table 5b. Sensitivity analysis correlation coefficient (studies including patients with confirmed food allergy)

\begin{tabular}{|c|c|c|c|c|c|c|c|c|c|}
\hline \multirow[t]{2}{*}{ Hourihane } & \multicolumn{3}{|c|}{$r=0.5$} & \multicolumn{3}{|c|}{$r=0.2$} & \multicolumn{3}{|c|}{$r=0.8$} \\
\hline & \multirow[t]{2}{*}{ SMD } & \multicolumn{2}{|c|}{$95 \% \mathrm{CI}$} & \multirow[t]{2}{*}{ SMD } & \multicolumn{2}{|c|}{$95 \%$ CI } & \multirow[t]{2}{*}{ SMD } & \multicolumn{2}{|c|}{$95 \% \mathrm{CI}$} \\
\hline \multicolumn{7}{|l|}{ FAQLQ-PF (0-1M) } & & & \\
\hline Total & $1.12^{*}$ & 1.00 & 1.25 & $1.12^{*}$ & 0.96 & 1.29 & $1.12^{*}$ & 1.04 & 1.21 \\
\hline \multicolumn{10}{|l|}{ FAQLQ-CF (0-1M) } \\
\hline Total & $2.00 *$ & 1.83 & 2.17 & $2.00^{*}$ & 1.78 & 2.22 & $2.00^{*}$ & 1.89 & 2.11 \\
\hline \multirow[t]{2}{*}{ Knibb } & \multicolumn{3}{|c|}{$r=0.5$} & \multicolumn{3}{|c|}{$r=0.2$} & \multicolumn{3}{|c|}{$r=0.8$} \\
\hline & SMD & \multicolumn{2}{|c|}{$95 \% \mathrm{CI}$} & SMD & \multicolumn{2}{|c|}{$95 \% \mathrm{CI}$} & SMD & \multicolumn{2}{|c|}{$95 \% \mathrm{CI}$} \\
\hline \multicolumn{10}{|l|}{ FAQL-PB (0-3 to $6 \mathrm{M})$} \\
\hline Total (with OFC) & 0.37 & 0.01 & 0.37 & -0.09 & -0.09 & 0.83 & 0.37 & 0.14 & 0.60 \\
\hline Total (without OFC) & 0.11 & -0.17 & 0.11 & -0.25 & -0.25 & 0.46 & 0.11 & -0.07 & 0.28 \\
\hline Total (with vs. without OFC) & 0.19 & -0.09 & 0.19 & -0.16 & -0.16 & 0.54 & 0.19 & 0.01 & 0.37 \\
\hline \multicolumn{10}{|l|}{ PedsQL ${ }^{\mathrm{TM}} 4.0$ (0-3 to $\left.6 \mathrm{M}\right)$} \\
\hline Total (with OFC) & 0.00 & -0.35 & 0.00 & -0.45 & -0.45 & 0.45 & 0.00 & -0.22 & 0.22 \\
\hline Total (without OFC) & 0.07 & -0.20 & 0.07 & -0.28 & -0.28 & 0.43 & 0.07 & -0.10 & 0.25 \\
\hline Total (with vs. without OFC) & -0.08 & -0.50 & -0.08 & -0.61 & -0.61 & 0.45 & -0.08 & -0.34 & 0.19 \\
\hline \multicolumn{10}{|c|}{ WHOQOL-BREF (0-3 to 6M) } \\
\hline Total (with OFC) & -0.02 & -0.38 & -0.02 & -0.47 & -0.47 & 0.42 & -0.02 & -0.25 & 0.20 \\
\hline Total (without OFC) & 0.06 & -0.21 & 0.06 & -0.29 & -0.29 & 0.42 & 0.06 & -0.12 & 0.24 \\
\hline Total (with vs. without OFC) & -0.09 & -0.65 & -0.09 & -0.80 & -0.80 & 0.62 & -0.09 & -0.44 & 0.27 \\
\hline
\end{tabular}

* significant change in $\mathrm{HRQL}$

Abbreviations: $\mathrm{Cl}$, confidence interval; FAQLQ, Food Allergy Quality of Life Questionnaire; PB, Parental Burden; PF, Parent Form; CF, Child Form; M, months; OFC, oral food challenge; PedsQL ${ }^{T M} 4.0$, Paediatric Quality of Life Inventory 4.0; $r$, correlation coefficient; SMD, standardized mean difference. WHOQOL$B R E F$, World Health Organization generic Quality of Life scale. 


\section{Supplemental references}

1. Soller L, Mill C, Wong T, et al. Determinants of Quality of life (QOL) Among Children Undergoing Oral Food Challenge (OFC) in Canada. J Allergy Clin Immunol. 2017;139(2):AB57.

2. Westwood M, Ramaekers B, Lang $\mathrm{S}$, et al. ImmunoCAP ISAC and microtest for multiplex allergen testing in people with difficult to manage allergic disease: A systematic review and cost analysis. Health Technol Assess (Rockv). 2016;(67):1-178.

3. Uehara H, Fujii Y, Sekimoto K, et al. Concerns and expectations of pediatric patients and parents with regards to oral food challenges. Allergy. 2016;71:118-272.

4. Danchin M, De Bono N, Allen K, Tang M, Hiscock H. Managing simple food allergy in community settings: A pilot study investigating a new model of care. J Paediatr Child Health. 2016;52(3):315-320.

5. Greenhawt MJ, Couch CE, Franxman TJ, Galvin AD. The relationship between self-efficacy, quality of life, and oral food challenge. J Allergy Clin Immunol. 2016;137(2):AB135.

6. Kajornrattana T, Sangsupawanich P, Yuenyongviwat A. Impact of parent-reported food allergies on children's growth and quality of life of the caregivers. J Allergy Clin Immunol. 2016;137(2):AB255.

7. Couch C, Franxman T, Greenhawt M. The economic effect and outcome of delaying oral food challenges. Ann Allergy Asthma Immunol. 2016;116(5):420-424.

8. Protudjer JLP, Jansson S a., Ostblom E, et al. Health-related quality of life in children with objectively diagnosed staple food allergy assessed with a disease-specific questionnaire. Acta Paediatr Int J Paediatr. 2015;104(10):1047-1054.

9. Venter $\mathrm{C}$, Sommer I, Moonesinghe $\mathrm{H}$, et al. Health-Related Quality of Life in children with perceived and diagnosed food hypersensitivity. Pediatr Allergy Immunol. 2015;26(2):126-132.

10. Howe L, Franxman T, Teich E, Greenhawt M. What affects quality of life among caregivers of food-allergic children? Ann Allergy Asthma Immunol. 2014;113(1):69-74.e2.

11. Le T-M, Flokstra-De Blok BMJ, Van Hoffen E, et al. Quality of life is more impaired in patients seeking medical care for food allergy. Int Arch Allergy Immunol. 2013;162(4):335-339.

12. Zurzolo GA, Allen KJ, Taylor SL, et al. Peanut Allergen Threshold Study (PATS): validation of eliciting doses using a novel single-dose challenge protocol. Allergy Asthma Clin Immunol. 2013;9(1):35.

13. Knibb RC, Ibrahim NF, Petley R, et al. Validation of the Paediatric Food Allergy Quality of Life Questionnaire (PFA-QL). Pediatr Allergy Immunol. 2013;24(3):288-292.

14. Brown T, Boyle R, Gore C, Warner J, Cox H. Effects of oral food challenges to selected nut(s) on quality of life and risk. Clin Exp Allergy. 2012;42:1815-1862.

15. Finger A, Pfannenstiel C, Schonfelder A, Frank F, Buderus S, Lange L. Improvement of quality of life in children with milk and egg allergy after proof of tolerance to baked products. Allergy. 2012;96:1-97.

16. Kirste L, Takaro TK, Kuzeljevic B, Wong T, Chan ES. Outcome Rather Than the Type of Diagnostic Intervention Predicts Improvement in Health-Related Quality of Life Score Among Children With Food Allergy Between 0-12 Years. J Allergy Clin Immunol. 2012;129(2):AB132.

17. Dubois AT. Benefits of effective diagnosis on impact of quality of life. Clin Transl Allergy. 2011;1(Suppl 1):2011.

18. Rotiroti G, Gardner J, Coppens K, Parker J. Usefulness of peanut and hazelnut molecular components for the diagnosis of nut allergy. Our experience in clinical practice. Clin Transl Allergy. 2011;1.

19. Paterakis M, Mudd K, Holbrook T, Page G, Gross D, Wood R. Do Peanut Oral Food Challenges Produce More Stress? Self-reported Anxiety Measurement Of Food Allergic Children And Their Parents Prior To Oral Food Challenges. J Allergy Clin Immunol. 2011;127(2):AB76.

20. van der Velde J, Flokstra-de Blok B , Vlieg-Boerstra J, Oude Elberink J, Duiverman E DA. Should Previous Responses Be Shown to Study Objects; A Comparison of Informed versus Blind 
Administration of the Food Allergy Quality of Life Questionnaire-Adult Form (FAQLQ-AF). J Allergy Clin Immunol. 2015;123(2):S25.

21. Flammarion S, Santos C, Romero D. Changes in diet and life of children with food allergies after a negative food challenge. Allergy. 2010;65:791-804.

22. Sohi D, Mkandawire $C$, James A, et al. Outcome of food challenges in children. Clin Exp Allergy. 2009;39(12):1937-1958.

23. Zijlstra WT, Flinterman $A E$, Soeters $L$, et al. Parental anxiety before and after food challenges in children with suspected peanut and hazelnut allergy. Pediatr Allergy Immunol. 2010;21:439-445.

24. Ostblom E, Egmar A-C, Gardulf A, Lilja G, Wickman M. The impact of food hypersensitivity reported in 9-year-old children by their parents on health-related quality of life. Allergy. 2008;63(2):211-218.

25. Polloni L, Ferruzza E, Ronconi L, et al. Assessment of children's nutritional attitudes before oral food challenges to identify patients at risk of food reintroduction failure: a prospective study. Allergy. 2017;72(5):731-736.

26. van der Valk JPM, Gerth van Wijk R, Flokstra-de Blok BMJ, et al. No difference in health-related quality of life, after a food challenge with cashew nut in children participating in a clinical trial. Pediatr Allergy Immunol. 2016;27(8):812-817.

27. Davis N, Egan M, Sicherer SH. Factors resulting in deferral of diagnostic oral food challenges. J Allergy Clin Immunol Pract. 2015;3(5):811-812.e1.

28. Franxman TJ, Howe L, Teich E, Greenhawt MJ. Oral Food Challenge and Food Allergy Quality of Life in Caregivers of Children with Food Allergy. J Allergy Clin Immunol Pract. 2014;3(1):50-56.

29. Soller L, Hourihane J, Dunngalvin A. The impact of oral food challenge tests on food allergy health-related quality of life. Allergy. 2014;69(9):1255-1257.

30. Herbert $\mathrm{L}$, Dahlquist LM, Bollinger ME. Maternal intolerance of uncertainty, anxiety, and adherence with food challenge referrals. J Health Psychol. 2013;18(9):1209-1219.

31. Knibb RC, Semper H. Impact of suspected food allergy on emotional distress and family life of parents prior to allergy diagnosis. Pediatr Allergy Immunol. 2013;24(8):798-803.

32. Van Der Velde JL, Flokstra-De Blok BMJ, De Groot $\mathrm{H}$, et al. Food allergy-related quality of life after double-blind, placebo-controlled food challenges in adults, adolescents, and children. $J$ Allergy Clin Immunol. 2012;130(5):1136-1143.e2.

33. Knibb RC, Ibrahim NF, Stiefel G, et al. The psychological impact of diagnostic food challenges to confirm the resolution of peanut or tree nut allergy. Clin Exp Allergy. 2012;42(3):451-459.

34. Kemp AS, Allen CW, Campbell DE. Parental perceptions in egg allergy: Does egg challenge make a difference? Pediatr Allergy Immunol. 2009;20(7):648-653.

35. Hayden JA, Coté $P$, Bombardier $C$. Evaluation of the quality of prognosis studies in systematic reviews. Ann Intern Med. 2006;144(6):427-437.

36. Hourihane JOB, Allen KJ, Shreffler WG, et al. Peanut Allergen Threshold Study (PATS): Novel single-dose oral food challenge study to validate eliciting doses in children with peanut allergy. J Allergy Clin Immunol. 2017;139(5):1583-1590.

37. Dunngalvin A, Cullinane C, Daly DA, Blok BMJF, Dubois AEJ, Hourihane JOB. Longitudinal validity and responsiveness of the Food Allergy Quality of Life Questionnaire - Parent Form in children $0-12$ years following positive and negative food challenges. Clin Exp Allergy. 2010:476-485. 


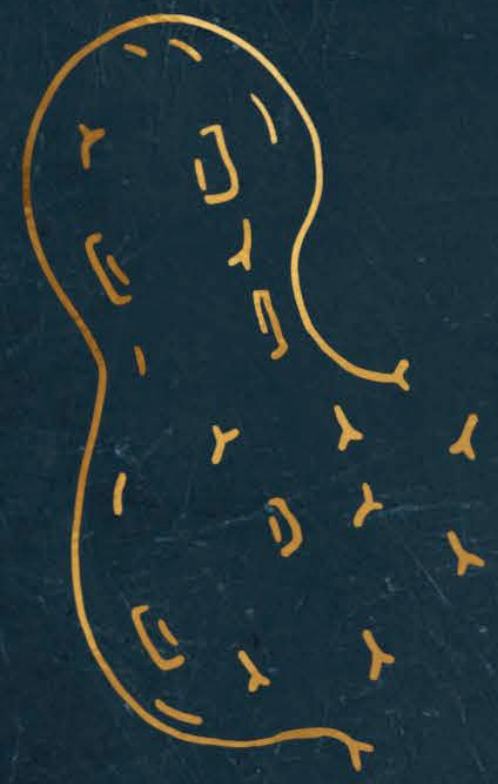

$+1$

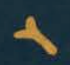

1

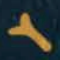




\section{Chapter 7}

\section{Prevalence and predictors of uncontrolled asthma in children referred for asthma and other atopic diseases}




\section{Abstract}

\section{Background}

Uncontrolled asthma in children is still highly prevalent despite the availability of effective asthma treatment. We investigated 1) the prevalence of uncontrolled asthma among children referred for asthma and referred for atopic diseases other than asthma (ie food allergy, allergic rhinitis, atopic dermatitis) to secondary care;

2) the predictors associated with uncontrolled asthma.

\section{Methods}

All children (4 to 18 years) referred for asthma or other atopic diseases than asthma to 8 secondary care centers in the Netherlands were invited for an Electronic Portal (EP). The EP is a web-based application with several validated questionnaires including the ISAAC questionnaires and the Asthma Control Test (ACT). Children were eligible for inclusion in this study when their parents reported in the EP that their child had asthma diagnosed by a physician. The ACT was used to assess asthma control. Multiple predictors of asthma control (patient, asthma and atopic characteristics) were evaluated by univariable and multivariable logistic regression analyses.

\section{Results}

We included 408 children: 259 children (63\%) with asthma referred for asthma and 149 children (37\%) with asthma referred for atopic diseases other than asthma. Thirty-nine percent of all children had uncontrolled asthma: $47 \%$ of children referred for asthma and $26 \%$ of children referred for atopic diseases other than asthma. Predictors associated with uncontrolled asthma were a family history of asthma (odds ratio [OR] 2.08; 95\% confidence interval [95\% CI] 1.34 to 3.24), recurrent upper and lower respiratory tract infections in the past year (OR 2.40; 95\% CI 1.52 to 3.81 and OR $2.00 ; 95 \%$ CI 1.25 to 3.23 , respectively).

\section{Conclusions}

Uncontrolled asthma is highly prevalent in children with asthma referred to secondary care, even if children are primarily referred for atopic diseases other than asthma. Thus, attention should be paid to asthma control in this population 


\section{Introduction}

Asthma is one of the most common chronic inflammatory diseases in the Western world, and a notable cause of morbidity in children. ${ }^{1}$ Good asthma control is the main management goal according to current asthma management guidelines, as this decreases the risk of asthma exacerbations and improves quality of life. ${ }^{2}$ However, uncontrolled asthma is still highly prevalent despite the availability of effective asthma treatment. ${ }^{3}$ In order to achieve adequate asthma control in children with asthma it is necessary to gain insight into the current prevalence of uncontrolled asthma among children and the associated predictors.

Several predictors of uncontrolled asthma in children have been previously described such as age, male gender, maternal education level, exposure to indoor smoking, pet ownership, high use of short-acting $\beta 2$-agonists, incorrect inhaler technique, poor adherence to asthma medication and the presence of co-existing diseases. ${ }^{2,4,5}$ Many children with asthma have co-existing atopic diseases - including food allergy, allergic rhinitis and atopic dermatitis - or recurrent respiratory tract infections. Food allergy has been reported in around $25 \%$ of children with asthma, allergic rhinitis in $60 \%$, atopic dermatitis in $45 \%$ and recurrent respiratory tract infections in $60 \% .{ }^{6-10}$ Atopic comorbidities and recurrent respiratory tract infections are associated with increased asthma morbidity. Children with asthma and food allergy have increased asthma symptoms and a significantly lower lung function compared to asthmatic children without food allergy. ${ }^{6,11}$ Children with asthma and allergic rhinitis have a lower level of asthma control compared to asthmatic children without allergic rhinitis. ${ }^{12,13}$ In addition, the presence of allergic rhinitis increases the risk of asthma exacerbations, emergency visits and hospitalizations. Furthermore, recurrent respiratory tract infections are associated with asthma exacerbations in children and a lower level of asthma control. ${ }^{14}$

Although predictors of uncontrolled asthma have been previously investigated in various studies, there are still some gaps in knowledge. The majority of research investigating asthma control is conducted among children referred for asthma ${ }^{4,15}$ or among children in the general population ${ }^{5,6,8,12}$ but not among children that are referred with atopic diseases other than asthma to secondary care. The aim of this cross-sectional study was 1 ) to investigate the prevalence of uncontrolled asthma among children referred for asthma and among children referred for atopic diseases other than asthma, and 2) to investigate the predictors, including atopic comorbidities, associated with uncontrolled asthma among these children. 


\section{Methods}

\section{Domain and data collection}

We conducted a cross-sectional study among children aged 4 to 18 years who were referred to a participating secondary care center $(n=8)$ in the Netherlands between June 2011 and December 2017. All children who were referred for asthma or for atopic diseases other than asthma (i.e. food allergy, allergic rhinitis, atopic dermatitis) were invited to fill in an Electronic Portal (EP) as part of their outpatient visit. There were no exclusion criteria for participation in the EP. Children were eligible for inclusion in this study when their parents reported in the EP that their child had a physician-diagnosed asthma. The structure of the EP have been published previously. ${ }^{16}$ In brief, the EP is a web-based application for children and their parents which has been developed and used by a nationwide collaborative network of Dutch caregivers in primary-, secondary- and tertiary health care. The aim of the EP is to collect information on asthma, atopic comorbidities and recurrent respiratory tract infections using validated questionnaires including the ISAAC core questionnaires for wheezing, allergic rhinitis, and atopic dermatitis, the Asthma Control Test, the Pediatric Asthma Quality of Life Questionnaire and the RAND general health questionnaire. ${ }^{17-22}$ The study has been approved by the Medical Ethics Committee of the University Medical Center Utrecht (No. 10/348) and all parents and/or children gave informed consent in the EP.

\section{Outcome}

Asthma control during the past month was assessed using the standardized and validated Child-Asthma Control Test (C-ACT) in children 4 to 12 years of age and the Asthma Control Test (ACT) in children $\geq 12$ years of age. ${ }^{18,19}$ The final asthma control score is the summed score ranging from 5 through 27 (C-ACT) or 5 through 25 (ACT) with higher scores indicating better asthma control. A score of $<20$ indicates uncontrolled asthma. ${ }^{18,19}$

\section{Potential predictors of asthma control}

Potential predictors of asthma control were selected based on clinical knowledge and prior research: age (4 to 12 years or 12 to 18 years), gender, maternal education level (categories low, middle or high), a family history of asthma in one or both parent(s), current smoke exposure, current pet ownership, use of daily inhaled corticosteroids (categories 'no', 'yes, adherent' or 'yes, non-adherent'), food allergy, allergic rhinitis, atopic dermatitis, recurrent upper respiratory tract infections in the past year, recurrent lower respiratory tract infections in the past year and the referral reason ('referral for asthma' or 'referral for atopic diseases other than asthma'). Food allergy was defined as self-reported relevant allergic complaints (urticaria, oral allergy symptoms, angioedema, upper or lower 
respiratory complaints, vomiting, a loss of consciousness or anaphylaxis) within two hours after ingestion of a food (yes or no). Allergic rhinitis was defined as having problems with sneezing, or a runny, or a blocked nose when the child did not have a cold or the flu, accompanied by itchy-watery eyes in the past 12 months (yes or no). Atopic dermatitis was defined as the presence of an itchy rash for six months or longer ever and complaints of an itchy rash in the past 12 months (yes or no). Recurrent upper respiratory tract infections included middle ear infections, croup, a cold or laryngitis and was defined as $\geq 8$ episodes/year for children aged 2 to 5 years, $\geq 6$ for ages $5-10$ years and $\geq 4$ for ages 10 years or older. Recurrent lower respiratory tract infections included severe bronchitis or pneumonia and was defined as $\geq 2$ episodes/year for children of all ages. Definitions of all predictors are displayed in Supplemental Table 1.

Adherence to asthma medication was assessed among all parents of children using daily inhaled corticosteroids by the Medication Adherence Rating Scale (MARS). ${ }^{23}$ The MARS is a 5 -item questionnaire on medication use behavior. Children with a total MARS score $\geq 21$ were considered adherent. ${ }^{24}$

\section{Statistical analysis}

Differences between children with complete and incomplete data and differences between children referred for asthma and referred for atopic diseases other than asthma were statistically evaluated by the chi-square test for categorical variables, the unpaired t-test for normally distributed continuous variables and the MannWhitney $U$ tests for non-normally distributed continuous variables with Bonferroni correction for multiple testing. Univariable and multivariable logistic regression analyses of all potential predictors as independent variables and asthma control (well-controlled versus uncontrolled) as the dependent variable were performed. We manually removed predictors one by one based on the $P$-value $(p>0.20$ based on the likelihood ratio test) and change in model fit (Akaike information criterion). ${ }^{25}$ The Odds Ratios (ORs) with 95\% confidence intervals (CIs) were estimated. A Pvalue $<0.05$ was considered statistically significant. In addition to backward selection, forward selection was conducted to evaluate whether the identified predictors were robust based on the sample. Both methods identified the same predictors. All analyses were performed in SPSS Statistics for Windows (Version 25.0. Armonk, NY: IMB Corp). 


\section{Results}

\section{Patients}

The study population flowchart is displayed in Figure 1. A total of 1676 children aged 4 to 18 years were invited for the Electronic Portal and 1556 (93\%) completed the screening questionnaire. Parents of 579 children reported their child to have asthma diagnosed by a physician. Asthma diagnosis was established by a pediatrician in 284 (49.1\%) children, by a general practitioner in 235 (40.6\%) children and by a pulmonologist in $60(10.4 \%)$ children. Complete data on the ACT and all predictors were available in 408 of 579 children (70\%). These children did not differ in the level of asthma control, the frequencies of atopic comorbidities and the frequencies of other predictors (data not shown). Only children with complete data were included in the analysis of asthma control.

\section{Asthma control in children referred for asthma or atopic diseases other than asthma}

Clinical characteristics of 408 children with asthma are displayed in Table 1 , stratified by the referral reason: 259 children (63\%) with asthma were referred for asthma and 149 children (37\%) with asthma were referred for atopic diseases other than asthma including food allergy, allergic rhinitis and atopic dermatitis. Children had a mean (SD) age of 10.6 (3.5) years and were predominantly Dutch (83\%). Uncontrolled asthma (ACT score < 20) was found in 160 children (39\%): in 121 of 259 children (47\%) referred for asthma and in 39 of 149 children (26\%) referred for atopic diseases other than asthma. Children referred for asthma were more likely to use daily inhaled corticosteroids compared to children referred for atopic diseases other than asthma ( $80 \%$ versus $56 \%$, respectively), and were less likely to report at least on other atopic comorbidity ( $61 \%$ versus $89 \%$, respectively). The proportion of atopic comorbidities in children in both referral groups is displayed in Figure 2. Almost $40 \%$ of children in both referral groups reported recurrent upper respiratory tract infections in the past year. 


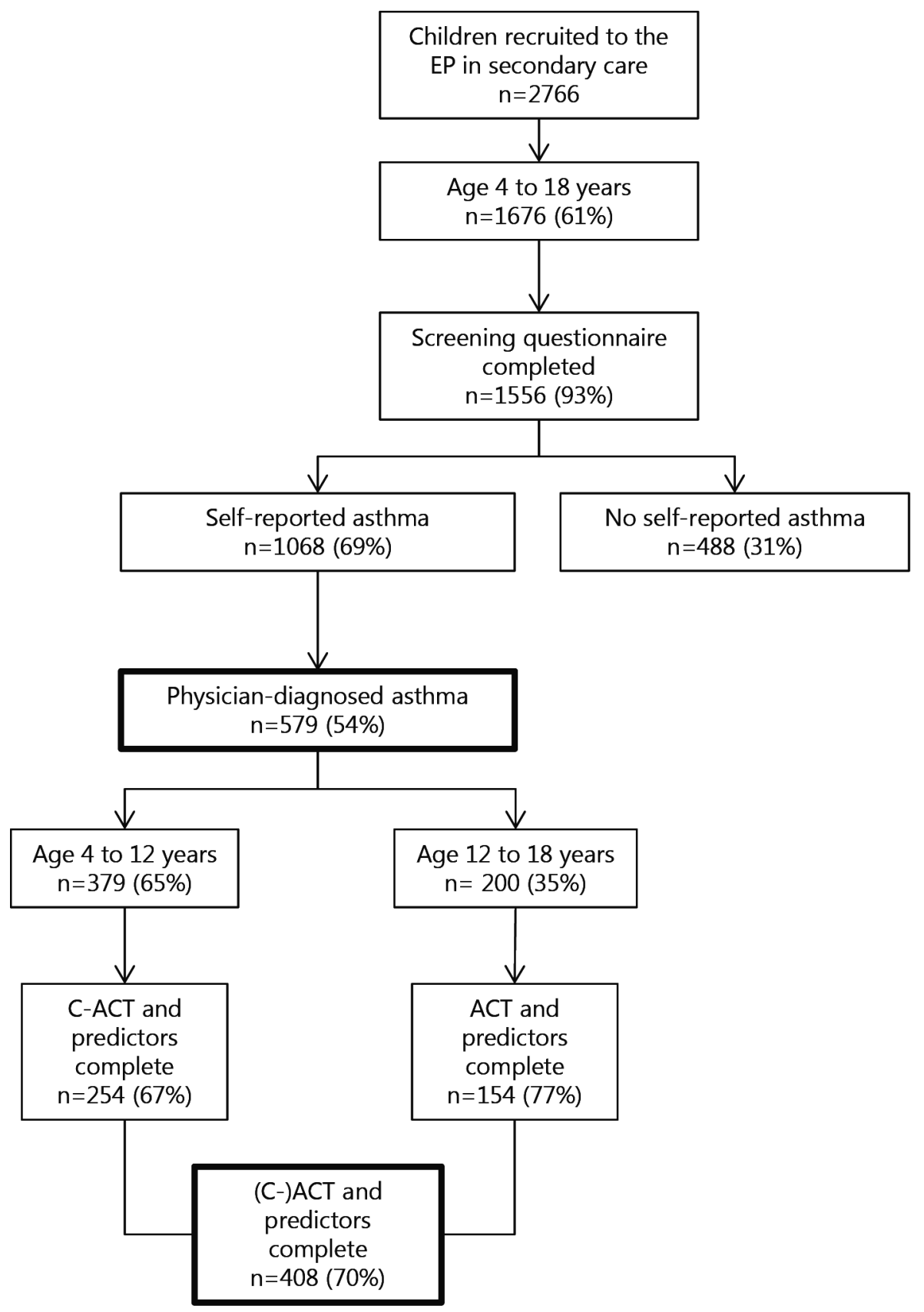

Figure 1. Study population flowchart

Abbreviations: (C-)ACT, (Child) Asthma Control Test; EP, Electronic Portal; n, number. 
Table 1. Clinical characteristics of children with asthma

\begin{tabular}{|c|c|c|c|c|}
\hline & \multirow[t]{2}{*}{ All children } & \multicolumn{2}{|c|}{ Referral reason } & \multirow[t]{2}{*}{$P$-value } \\
\hline & & Asthma & $\begin{array}{l}\text { Atopic disease } \\
\text { other than } \\
\text { asthma }\end{array}$ & \\
\hline $\mathrm{n}(\%)$ & $408(100)$ & $259(63)$ & $149(37)$ & \\
\hline \multicolumn{5}{|l|}{ Demographic characteristics } \\
\hline Age in years, mean (SD) & $10.6(3.5)$ & $10.3(3.4)$ & $11.2(3.6)$ & 0.021 \\
\hline Male gender & $238(58)$ & $160(62)$ & $78(52)$ & 0.076 \\
\hline Both parents Dutch & $340(83)$ & $210(81)$ & $130(87)$ & 0.129 \\
\hline Family history of asthma & $198(49)$ & $124(48)$ & $74(50)$ & 0.758 \\
\hline Family history of other atopic disease & $331(81)$ & $205(79)$ & $126(85)$ & 0.191 \\
\hline Current smoke exposure & $31(8)$ & $17(7)$ & $14(9)$ & 0.334 \\
\hline Current pet ownership & $165(40)$ & $105(41)$ & $60(40)$ & 1.000 \\
\hline \multicolumn{5}{|l|}{ Asthma characteristics } \\
\hline Wheezing at night (past year) & $173(42)$ & $124(48)$ & $49(33)$ & 0.004 \\
\hline Coughing at night (past year) & $196(48)$ & $132(51)$ & $64(43)$ & 0.124 \\
\hline $\begin{array}{l}\text { Inhaled short-acting } \beta 2 \text {-agonists (past } \\
\text { week) }\end{array}$ & $228(56)$ & $158(61)$ & $70(47)$ & 0.007 \\
\hline Inhaled corticosteroids (daily) $^{\ddagger}$ & & & & $<0.001$ \\
\hline No & $118(29)$ & $53(21)$ & $65(44)$ & \\
\hline Yes, adherent & $213(52)$ & $154(60)$ & $59(40)$ & \\
\hline Yes, non-adherent & 77 (19) & $52(20)$ & $25(16)$ & \\
\hline \multicolumn{5}{|l|}{ (Atopic) comorbidities } \\
\hline Any atopic comorbidity & $291(71)$ & $159(61)$ & $132(89)$ & $<0.001$ \\
\hline Food allergy & $87(21)$ & $30(12)$ & $57(38)$ & $<0.001$ \\
\hline Allergic rhinitis & $200(49)$ & $100(39)$ & $100(67)$ & $<0.001$ \\
\hline Atopic dermatitis & $173(42)$ & $91(35)$ & $82(55)$ & $<0.001$ \\
\hline Recurrent upper RTIs (yes) & $146(36)$ & $89(34)$ & $57(38)$ & 0.454 \\
\hline Recurrent lower RTIs (yes) & $116(28)$ & $82(32)$ & $34(23)$ & 0.068 \\
\hline \multicolumn{5}{|l|}{ Quality of life } \\
\hline PAQLQ, median (IQR) & $6.1(5.4-6.7)$ & $5.9(5.0-6.6)$ & $6.2(5.6-6.8)$ & 0.003 \\
\hline RAND, mean (SD) & $21(4.6)$ & $21(4.5)$ & $21(4.7)$ & 0.991 \\
\hline \multicolumn{5}{|l|}{ Referral reason } \\
\hline Asthma & $259(64)$ & $259(100)$ & 0 & \\
\hline Food allergy & $73(18)$ & 0 & $73(49)$ & \\
\hline Allergic rhinitis & $38(9)$ & 0 & $38(26)$ & \\
\hline Atopic dermatitis & $27(7)$ & 0 & $27(18)$ & \\
\hline Other, none of the above & $11(3)$ & 0 & $11(7)$ & \\
\hline
\end{tabular}

Values are numbers (\%), unless otherwise stated.

${ }^{+}$Statistical comparisons between referral groups with $P$-values of less than 0.003 taken to be significant according to Bonferroni correction for multiple testing.

${ }^{\ddagger}$ Medication adherence was assessed by the Medication Adherence Rating Scale (MARS). ${ }^{23}$ Children with a total MARS score $\geq 21$ were considered adherent and a score $<21$ were considered non-adherent. ${ }^{24}$

Abbreviations: IQR, interquartile range; $R T I s$, respiratory tract infections; $n$, number; $N A$, not applicable; $S D$, standard deviation. 
Children referred for asthma $(n=259)$
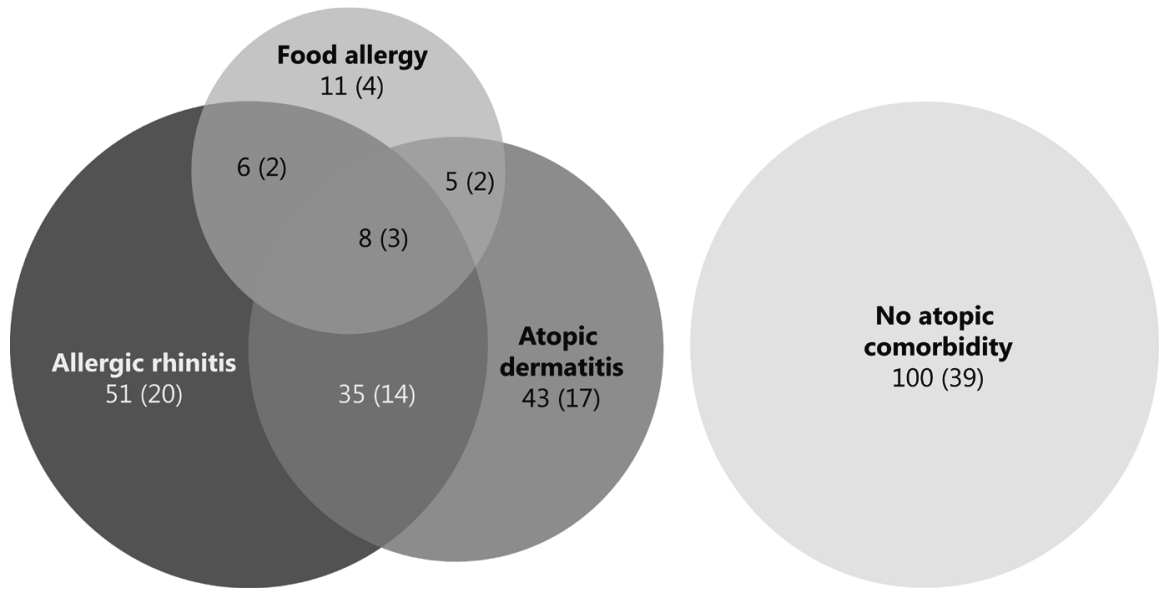

Children referred for atopic diseases other than asthma $(n=149)$

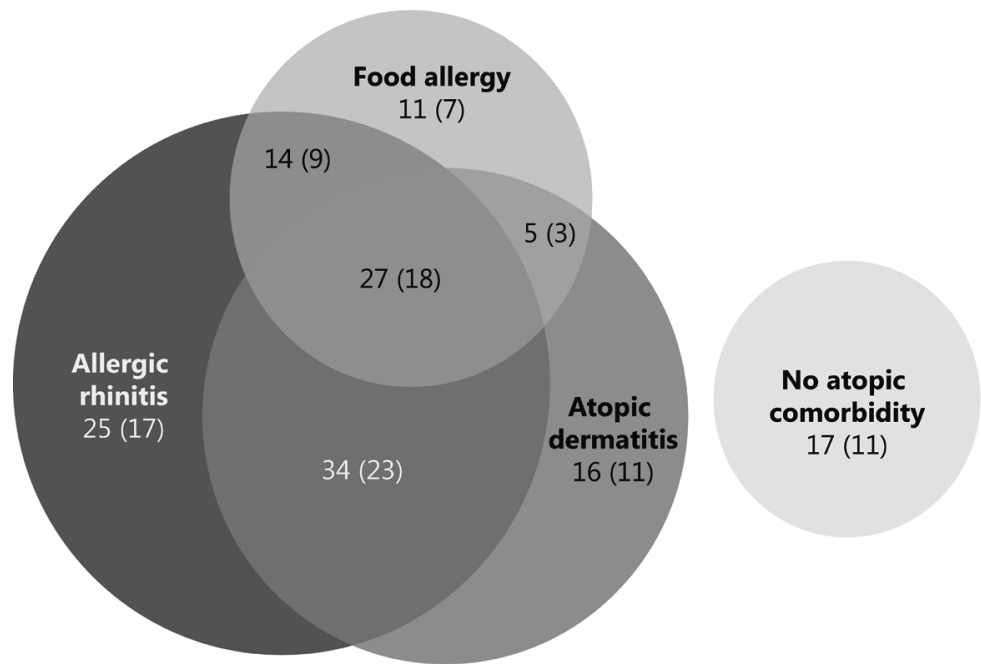

Figure 2. Venn diagram displaying the proportion of atopic comorbidities among children with asthma stratified by referral reason

Values are numbers (\%).

\section{Predictors of asthma control}

Univariable logistic regression analyses showed that a family history of asthma, the use of daily inhaled corticosteroids in children who were considered non-adherent, recurrent upper and lower respiratory tract infections in the past year and a referral for asthma were associated with uncontrolled asthma (Table 2). In a multivariable logistic regression analysis, a family history of asthma (odds ratio [OR] 2.08; $95 \% \mathrm{CI}$ 1.34 to 3.24), recurrent upper respiratory tract infections in the past year (OR 2.40; $95 \% \mathrm{CI} 1.52$ to 3.81 ), recurrent lower respiratory tract infections in the past year (OR 
2.00; $95 \%$ CI 1.25 to 3.23 ) and a referral for asthma (OR 2.60; $95 \%$ CI 1.61 to 4.26 ) were independently and significantly associated with uncontrolled asthma (Table 2).

Uncontrolled asthma was observed in $47 \%$ of children with a family history of asthma versus $32 \%$ of children without a family history of asthma, in $51 \%$ of children with recurrent upper RTIs versus $32 \%$ of children without recurrent upper RTIs and in 53\% of children with recurrent lower RTIs versus $34 \%$ of children without recurrent lower RTIs. The full multivariable logistic regression analysis is presented in Supplemental Table 2. Atopic comorbidities, including food allergy, allergic rhinitis and atopic dermatitis, were not associated with uncontrolled asthma.

Table 2. Univariable and fitted multivariable logistic regression analysis, with uncontrolled asthma (ACT score $<20$ ) as the outcome, in 408 children with asthma

\begin{tabular}{|c|c|c|c|c|}
\hline & \multicolumn{2}{|c|}{ Univariable analysis } & \multicolumn{2}{|c|}{ Fitted multivariable analysis $^{\dagger}$} \\
\hline & $\begin{array}{l}\text { Crude } \\
\text { OR }(95 \% \text { CI })\end{array}$ & $P$-value & $\begin{array}{l}\text { Adjusted } \\
\text { OR }(95 \% \text { CI })\end{array}$ & $P$-value \\
\hline Age category & & 0.616 & & \\
\hline 4 to 12 year & $\operatorname{Ref}$ & & & \\
\hline 12 to 18 year & $0.90(0.60$ to 1.36$)$ & & & \\
\hline Male gender & $1.45(0.97$ to 2.18$)$ & 0.074 & $1.50(0.96$ to 2.37$)$ & 0.073 \\
\hline Maternal education level & & 0.196 & & 0.099 \\
\hline Low & $\operatorname{Ref}$ & & $\operatorname{Ref}$ & \\
\hline Middle & $1.52(0.81$ to 2.91$)$ & & $1.60(0.81$ to 3.23$)$ & \\
\hline High & $1.06(0.57$ to 2.03$)$ & & $0.98(0.50$ to 1.98$)$ & \\
\hline Family history of asthma & 1.81 (1.21 to 2.72$)$ & 0.004 & 2.08 (1.34 to 3.24$)$ & 0.001 \\
\hline Current smoke exposure & $1.73(0.82$ to 3.63$)$ & 0.146 & & \\
\hline Current pet ownership & $1.42(0.95$ to 2.13$)$ & 0.087 & 1.51 (0.97 to 2.36$)$ & 0.066 \\
\hline Inhaled corticosteroids (daily) $^{\ddagger}$ & & 0.027 & & 0.147 \\
\hline No & $\operatorname{Ref}$ & & $\operatorname{Ref}$ & \\
\hline Yes, adherent & 1.54 (0.96 to 2.50$)$ & & 1.05 (0.62 to 1.79$)$ & \\
\hline Yes, non-adherent & 2.22 (1.23 to 4.04$)$ & & $1.78(0.93$ to 3.44$)$ & \\
\hline Food allergy & $0.52(0.30$ to 0.86$)$ & 0.011 & & \\
\hline Allergic rhinitis & $0.87(0.58$ to 1.29$)$ & 0.486 & & \\
\hline Atopic dermatitis & 0.85 (0.57 to 1.27$)$ & 0.430 & & \\
\hline Recurrent upper RTIs (yes) & $2.20(1.45$ to 3.34$)$ & $<0.001$ & $2.40(1.52$ to 3.81$)$ & $<0.001$ \\
\hline Recurrent lower RTIs (yes) & 2.27 (1.47 to 3.53$)$ & $<0.001$ & 2.00 (1.25 to 3.23$)$ & 0.004 \\
\hline Referral reason & & $<0.001$ & & $<0.001$ \\
\hline $\begin{array}{l}\text { Atopic diseases } \\
\text { other than asthma }\end{array}$ & $\operatorname{Ref}$ & & $\operatorname{Ref}$ & \\
\hline Asthma & 2.47 (1.60 to 3.87$)$ & & 2.60 (1.61 to 4.26$)$ & \\
\hline
\end{tabular}

${ }^{\dagger}$ Fitted multivariable logistic regression model after backward selection.

${ }^{\ddagger}$ Medication adherence was assessed by the Medication Adherence Rating Scale (MARS). ${ }^{23}$ Children with a total MARS score $\geq 21$ were considered adherent and a score $<21$ were considered non-adherent. ${ }^{24}$ Abbreviations: ACT, Asthma Control Test; $C l$, confidence interval; RTIs, respiratory tract infections; $n$, number; OR, odds ratio; Ref, Reference category. 


\section{Discussion}

Our study indicates that a high percentage of uncontrolled asthma is not only observed in children with asthma who were referred for asthma to secondary care (47\%) but uncontrolled asthma is also highly prevalent in children with asthma who were referred for atopic diseases other than asthma (26\%). The prevalence of uncontrolled asthma in our population of children with asthma referred for atopic diseases other than asthma is notably higher than the prevalence observed in children with asthma among the general population (15\%). ${ }^{5}$ In addition, only $56 \%$ of children with asthma referred for atopic diseases other than asthma were prescribed daily inhaled corticosteroids suggesting these children were inappropriately treated. ${ }^{26}$ Our results suggest that assessment of asthma control and optimization of asthma treatment are not only important for children referred for asthma, but also for children primarily referred for allergic complaints other than asthma.

We found a family history of asthma, recurrent upper and lower respiratory tract infections in the past year to be independently associated with uncontrolled asthma. Children with a family history of asthma might have more frequent uncontrolled asthma due to increased asthma severity, as suggested in previous research. ${ }^{27}$ Furthermore, children with a family history of asthma might have more frequent uncontrolled asthma as parents with asthma may be less concerned about the asthma symptoms of their child as they are familiar with asthma symptoms themselves. Finally, recurrent upper and lower respiratory tract infections were associated with uncontrolled asthma. This observation is concordant with previous studies that showed that respiratory tract infections can have a profound effect on loss of control and asthma exacerbations in both children and adults. ${ }^{28}$ Furthermore, asthma attacks are often triggered by respiratory tract infections. Another explanation for the observed association between respiratory tract infections and uncontrolled asthma might be that asthma has been misdiagnosed. ${ }^{29}$ Previous studies indicate that parent-reported physiciandiagnosed asthma in children is often not confirmed upon lung function testing and respiratory symptoms were due to e.g. viral illnesses. ${ }^{29,30}$

Atopic comorbidities, i.e. food allergy, allergic rhinitis and atopic dermatitis, were not independently associated with asthma control in our population of children referred with respiratory or allergic complaints. Previous research in children with asthma reported an increased risk of uncontrolled asthma and increased asthma morbidity (i.e. hospitalization, use of controller medication) in children with asthma and food allergy ${ }^{6}$ or allergic rhinitis. ${ }^{4,5}$ We might not have detected an association between atopic comorbidities and asthma control because the atopic comorbidities 
were self-reported. Furthermore, most studies focused on an individual comorbidity and included additional relevant predictors associated with the comorbidity in the analysis, e.g. the season of the questionnaire, the severity of the atopic comorbidity or medication use. The sample size of our study was too small to consider all these additional relevant predictors per atopic comorbidity in the regression model. This may explain why the association was not found.

The major limitation of the present study is that all data were self-reported, lacking objective data such as lung function testing or specific IgE measurement. The diagnosis of asthma was based on a parent-reported physician-diagnosis and the diagnoses of atopic comorbidities were mainly based on the ISAAC questionnaire. Therefore, the prevalence of these diseases may have been overestimated ${ }^{31,32}$ or underestimated. ${ }^{33}$ In addition, we only included the children with complete data which may have caused selection bias. However, the children with complete data were comparable to the children with incomplete data in the frequencies of all predictors and the level of asthma control providing confidence that the children included in the study are a fair representation of the overall study population. Finally, we did not assess long-term asthma control and the frequency of asthma exacerbations, emergency department visits and hospitalizations. However, our study is strengthened by the inclusion of a large population of children with parent-reported physician-diagnosed asthma in eight secondary care centers in the Netherlands, rigorous data collection using several validated questionnaires in children and parents, as well as the assessment of asthma, atopic comorbidities and respiratory tract infections simultaneously.

In conclusion, this study demonstrates that assessment of asthma control is not only important for all children referred for asthma to secondary care, but also for children with asthma who are referred for atopic diseases other than asthma. Special attention should be paid to children with asthma who have a family history of asthma, or have a history of recurrent upper and/or lower respiratory tract infections in the past year, as these predictors are associated with uncontrolled asthma.

\section{Acknowledgments}

The authors would like to thank the members of the Expert Network Frank Eskes, René van Gent and Arnold Ketel. Furthermore, the authors would like to thank Paco Welsing for his statistical advice and Lilly Verhagen for her help to define recurrent respiratory tract infections in children. 


\section{References}

1. Eder W, Ege MJ, von Mutius E. The Asthma Epidemic. N Engl J Med. 2006;355(21):2226-2235.

2. Global Initiative for Asthma (GINA). Global Strategy for Asthma Management and Prevention. Available from: https://ginasthma.org (2018). Accessed Aug 8, 2018.

3. Rabe KF, Adachi M, Lai CKW, et al. Worldwide severity and control of asthma in children and adults: The global asthma insights and reality surveys. J Allergy Clin Immunol. 2004;114(1):4047.

4. Oka A, Hirano T, Yamaji Y, et al. Determinants of Incomplete Asthma Control in Patients with Allergic Rhinitis and Asthma. J Allergy Clin Immunol Pract. 2017;5(1):160-164.

5. Sasaki M, Yoshida K, Adachi Y, et al. Factors associated with asthma control in children: Findings from a national Web-based survey. Pediatr Allergy Immunol. 2014;25(8):804-809.

6. Friedlander JL, Sheehan WJ, Baxi SN, Kopel LS. Food Allergy and Increased Asthma Morbidity in a School-Based Inner-City Asthma Study. J Allergy Clin Immunol Pract. 2013;1(5):479-484.

7. Leynaert B, Neukirch F, Demoly P, Bousquet J. Epidemiologic evidence for asthma and rhinitis comorbidity. J Allergy Clin Immunol. 2000;106(5):S201-S205.

8. Ballardini N, Kull I, Lind T, et al. Development and comorbidity of eczema, asthma and rhinitis to age 12 - Data from the BAMSE birth cohort. Allergy. 2012;67(4):537-544.

9. de Oliveira $T B$, Klering $E A$, da Veiga $A B G$. Is recurrent respiratory infection associated with allergic respiratory disease? J Asthma. 2019;56(2):160-166.

10. Tay TR, Hew M. Comorbid "treatable traits" in difficult asthma: Current evidence and clinical evaluation. Allergy. 2018;73(7):1369-1382.

11. Simpson $A B$, Glutting $J$, Yousef E. Food allergy and asthma morbidity in children. Pediatr Pulmonol. 2007;42(6):489-495.

12. Deliu M, Belgrave D, Simpson A, Murray CS, Kerry G, Custovic A. Impact of rhinitis on asthma severity in school-age children. Allergy Eur J Allergy Clin Immunol. 2014;69(11):1515-1521.

13. Bousquet J, Khaltaev N, Cruz AA, et al. Allergic Rhinitis and its Impact on Asthma (ARIA) 2008 update (in collaboration with the World Health Organization, GA2LEN and AllerGen). Allergy Eur J Allergy Clin Immunol. 2008;63(Suppl. 86):8-160.

14. Edwards MR, Strong K, Cameron A, Walton RP, Jackson DJ, Johnston SL. Viral infections in allergy and immunology: How allergic inflammation influences viral infections and illness. $J$ Allergy Clin Immunol. 2017;140(4):909-920.

15. Franklin JM, Grunwell JR, Bruce AC, Smith RC, Fitzpatrick AM. Predictors of emergency department use in children with persistent asthma in metropolitan Atlanta, Georgia. Ann Allergy, Asthma Immunol. 2017;119(2):129-136.

16. Zomer-Kooijker K, van Erp FC, Balemans WAF, van Ewijk BE, van der Ent CK. The expert network and electronic portal for children with respiratory and allergic symptoms: Rationale and design. BMC Pediatr. 2013;13(1):9.

17. Asher MI, Keil U, Anderson HR, et al. International study of asthma and allergies in childhood (ISAAC): Rationale and methods. Eur Respir J. 1995;8(3):483-491.

18. Nathan RA, Sorkness CA, Kosinski M, et al. Development of the Asthma Control Test: A survey for assessing asthma control. J Allergy Clin Immunol. 2004;113:59-65.

19. Liu AH, Zeiger R, Sorkness $C$, et al. Development and cross-sectional validation of the Childhood Asthma Control Test. J Allergy Clin Immunol. 2007;119:817-825.

20. Raat H, Bueving HJ, De Jongste JC, Grol MH, Juniper EF, Van Der Wouden JC. Responsiveness, longitudinal- and cross-sectional construct validity of the Pediatric Asthma Quality of Life Questionnaire (PAQLQ) in Dutch children with asthma. Qual Life Res. 2005;14(1):265-272.

21. Lewis CC, Pantell RH, Kiekhefer GM. Assessment of Children's Health Status: Field Test of New Approaches. Med Care. 1989;27(3 Suppl):S54-S65.

22. Post M, Kuyenhoven M, Verheij M, de Melker R, Hoes A. The Dutch "Rand General Health Rating Index for Children": a questionnaire measuring the general health status of children. Ned 
Tijdschr Geneeskd. 1998;142(49):2680-2683.

23. Cohen JL, Mann DM, Wisnivesky JP, et al. Assessing the validity of self-reported medication adherence among inner-city asthmatic adults: the Medication Adherence Report Scale for Asthma. Ann Allergy, Asthma Immunol. 2009;103(4):325-331.

24. Koster ES, Raaijmakers JAM, Vijverberg SJH, Maitland van der Zee AH. Inhaled corticosteroid adherence in paediatric patients: the PACMAN cohort study. Pharmacoepidemiol Drug Saf. 2011;20:1064-1072.

25. Hilbe JM. Logistic Regression. Springer Berlin Heidelberg, Berlin, Heidelberg; 2011.

26. Bateman ED, Hurd SS, Barnes PJ, et al. Global strategy for asthma management and prevention: GINA executive summary. European Respiratory Journal. 2008;31:143-178

27. Zhao J, He Q, Zhang G, et al. Status of asthma control in children and the effect of parents' knowledge, attitude, and practice (KAP) in China: A multicenter study. Ann Allergy, Asthma Immunol. 2012;109(3):190-194.

28. Busse WW, Lemanske Jr. RF, Gern JE. The Role of Viral Respiratory Infections in Asthma and Asthma Exacerbations. Lancet. 2010;376(9743):826-834.

29. Yang $C L$, Simons $E$, Foty RG, Subbarao $P$, To $T$, Dell SD. Misdiagnosis of asthma in schoolchildren. Pediatr Pulmonol. 2017;52(3):293-302.

30. Looijmans-van den Akker I, van Luijn K, Verheij T. Overdiagnosis of asthma in children in primary care: a retrospective analysis. Br J Gen Pract. 2016;66(644):e152-e157.

31. Hederos CA, Hasselgren M, Hedlin G, Bornehag CG. Comparison of clinically diagnosed asthma with parental assessment of children's asthma in a questionnaire. Pediatr Allergy Immunol. 2007;18(2):135-141.

32. Nwaru BI, Hickstein L, Panesar SS, et al. The epidemiology of food allergy in Europe: A systematic review and meta-analysis. Allergy. 2014;69(1):62-75.

33. Van Gent R, Van Essen-Zandvliet LEM, Rovers MM, Kimpen JLL, De Meere G, Van Der Ent CK. Poor perception of dyspnoea in children with undiagnosed asthma. Eur Respir J. 2007;30(5):887-891. 


\section{Supplemental tables}

Supplemental Table 1. Collection of clinical data using the Electronic Portal

\begin{tabular}{|c|c|c|}
\hline Variable & Question in the Electronic Portal & $\begin{array}{l}\text { Variable categories } \\
\text { (answer option questions } \\
\text { in Electronic Portal) }\end{array}$ \\
\hline $\begin{array}{l}\text { Physician- } \\
\text { diagnosed } \\
\text { asthma }\end{array}$ & $\begin{array}{l}\text { Is your child diagnosed with asthma? } \\
\text { a. Yes, by a general practitioner } \\
\text { b. Yes, by a pediatrician } \\
\text { c. Yes, by a pulmonologist } \\
\text { d. No }\end{array}$ & Yes $(a, b$ or $c)$ or No $(d)$ \\
\hline $\begin{array}{l}\text { Both parents } \\
\text { Dutch }\end{array}$ & 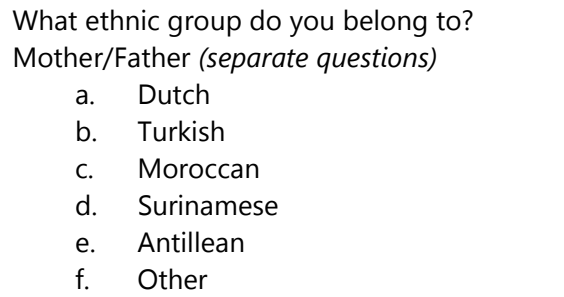 & Yes (both parents a) or No \\
\hline $\begin{array}{l}\text { Maternal } \\
\text { education level }\end{array}$ & $\begin{array}{l}\text { What is the highest level of education you } \\
\text { have completed? } \\
\text { a. Elementary school } \\
\text { b. Lower general secondary } \\
\text { c. Intermediate vocational education } \\
\text { d. Higher general secondary } \\
\text { e. Higher vocational education } \\
\text { f. University }\end{array}$ & $\begin{array}{l}\text { Low (a-b), Middle (c-d) or } \\
\text { High (e-f) }\end{array}$ \\
\hline $\begin{array}{l}\text { Family history of } \\
\text { asthma }\end{array}$ & $\begin{array}{l}\text { Do one or more family members suffer from } \\
\text { asthma, allergic rhinitis, food allergy or } \\
\text { atopic dermatitis? (four separate questions) } \\
\text { a. No } \\
\text { b. Yes, the father of the child } \\
\text { c. Yes, the mother of the child } \\
\text { d. Yes, brother(s) of the child } \\
\text { e. Yes, sister(s) of the child }\end{array}$ & Yes ( $b$ and/or $c$ ) or No \\
\hline $\begin{array}{l}\text { Family history of } \\
\text { other atopic } \\
\text { diseases }\end{array}$ & $\begin{array}{l}\text { Do one or more family members suffer from } \\
\text { food allergy, allergic rhinitis or atopic } \\
\text { dermatitis? (three separate questions) } \\
\text { a. No } \\
\text { b. Yes, the father of the child } \\
\text { c. Yes, the mother of the child } \\
\text { d. Yes, brother(s) of the child } \\
\text { e. Yes, sister(s) of the child }\end{array}$ & Yes ( $b$ and/or $c$ ) or No \\
\hline
\end{tabular}


Supplemental Table 1. Collection of clinical data using the Electronic Portal (continued)

\begin{tabular}{|c|c|c|}
\hline Variable & Question in the Electronic Portal & $\begin{array}{l}\text { Variable categories } \\
\text { (answer option questions } \\
\text { in Electronic Portal) }\end{array}$ \\
\hline $\begin{array}{l}\text { Current smoke } \\
\text { exposure }\end{array}$ & $\begin{array}{l}\text { 1. Does the mother of the child } \\
\text { smoke in the home? } \\
\text { 2. Does the father of the child } \\
\text { smoke in the home? } \\
\text { 3. Do other housemates smoke in } \\
\text { the home? }\end{array}$ & $\begin{array}{l}\text { Yes (question } 1,2 \text { or } 3 \text { yes) } \\
\text { or No }\end{array}$ \\
\hline $\begin{array}{l}\text { Current pet } \\
\text { ownership }\end{array}$ & Do you own a pet? & Yes or no \\
\hline $\begin{array}{l}\text { Wheezing/cough } \\
\text { ing at night } \\
\text { during the past } \\
\text { year }\end{array}$ & $\begin{array}{l}\text { 1. In the past } 12 \text { months, how often, } \\
\text { on average, has your child's sleep } \\
\text { been disturbed due to wheezing? } \\
\text { 2. In the past } 12 \text { months, has your } \\
\text { child had a dry cough at night, } \\
\text { apart from a cough associated } \\
\text { with a cold or a chest infection? }\end{array}$ & $\begin{array}{l}\text { Yes* or No } \\
\text { * Yes if question } 1 \text { is } \\
\text { answered with } \geq 1 \text {, and/or } \\
\text { question } 2 \text { answered with } \\
\text { yes }\end{array}$ \\
\hline $\begin{array}{l}\text { Inhaled short- } \\
\text { acting } \beta 2 \text { - } \\
\text { agonists (past } \\
\text { week) }\end{array}$ & $\begin{array}{l}\text { In the past week, how often has your child } \\
\text { inhaled a short-acting bronchodilator (e.g. } \\
\text { Ventolin, Salbutamol, Bricanyl) on most days } \\
\text { a. Never } \\
\text { b. } \quad 1-2 \text { inhalations on most days } \\
\text { c. } \quad 3-4 \text { inhalations on most days } \\
\text { d. } 5-8 \text { inhalations on most days } \\
\text { e. } 9-12 \text { inhalations on most days } \\
\text { f. } 13-16 \text { inhalations on most days } \\
\text { g. } \geq 16 \text { inhalations on most days }\end{array}$ & Yes (b-g) or No (a) \\
\hline $\begin{array}{l}\text { Inhaled } \\
\text { corticosteroids } \\
\text { (daily) }\end{array}$ & $\begin{array}{l}\text { Has your child used daily inhaled } \\
\text { corticosteroids (e.g. Pulmicort, Flixotide, } \\
\text { Seretide, Qvar)? } \\
\text { a. Yes } \\
\text { b. No } \\
\text { 5-item Medication Adherence Rating Scale } \\
\text { (MARS) }^{23}\end{array}$ & $\begin{array}{l}\text { No }(b) \text {, Yes adherent (a } \\
\text { and MARS } \geq 21 \text { ), Yes non- } \\
\text { adherent (a and MARS } \\
<21 \text { ) }\end{array}$ \\
\hline Food allergy & $\begin{array}{l}\text { 1. Has your child ever had a food } \\
\text { allergy? } \\
\text { 2. Within what time period after } \\
\text { food ingestion did your child } \\
\text { develop symptoms? } \\
\text { 3. What symptoms did your child } \\
\text { experience after food ingestion? }\end{array}$ & $\begin{array}{l}\text { Yes* or No } \\
\text { * Yes if question } 1 \text { is } \\
\text { answered with yes, and } \\
\text { symptoms occurred within } \\
\text { two hours after ingestion } \\
\text { of the food (question 2) } \\
\text { and food allergic } \\
\text { symptoms were relevant } \\
\text { (question 3): urticaria, oral } \\
\text { allergy, angioedema, } \\
\text { upper or lower respiratory } \\
\text { complaints, vomiting, loss } \\
\text { of consciousness or } \\
\text { anaphylaxis. }\end{array}$ \\
\hline
\end{tabular}


Supplemental Table 1. Collection of clinical data using the Electronic Portal (continued)

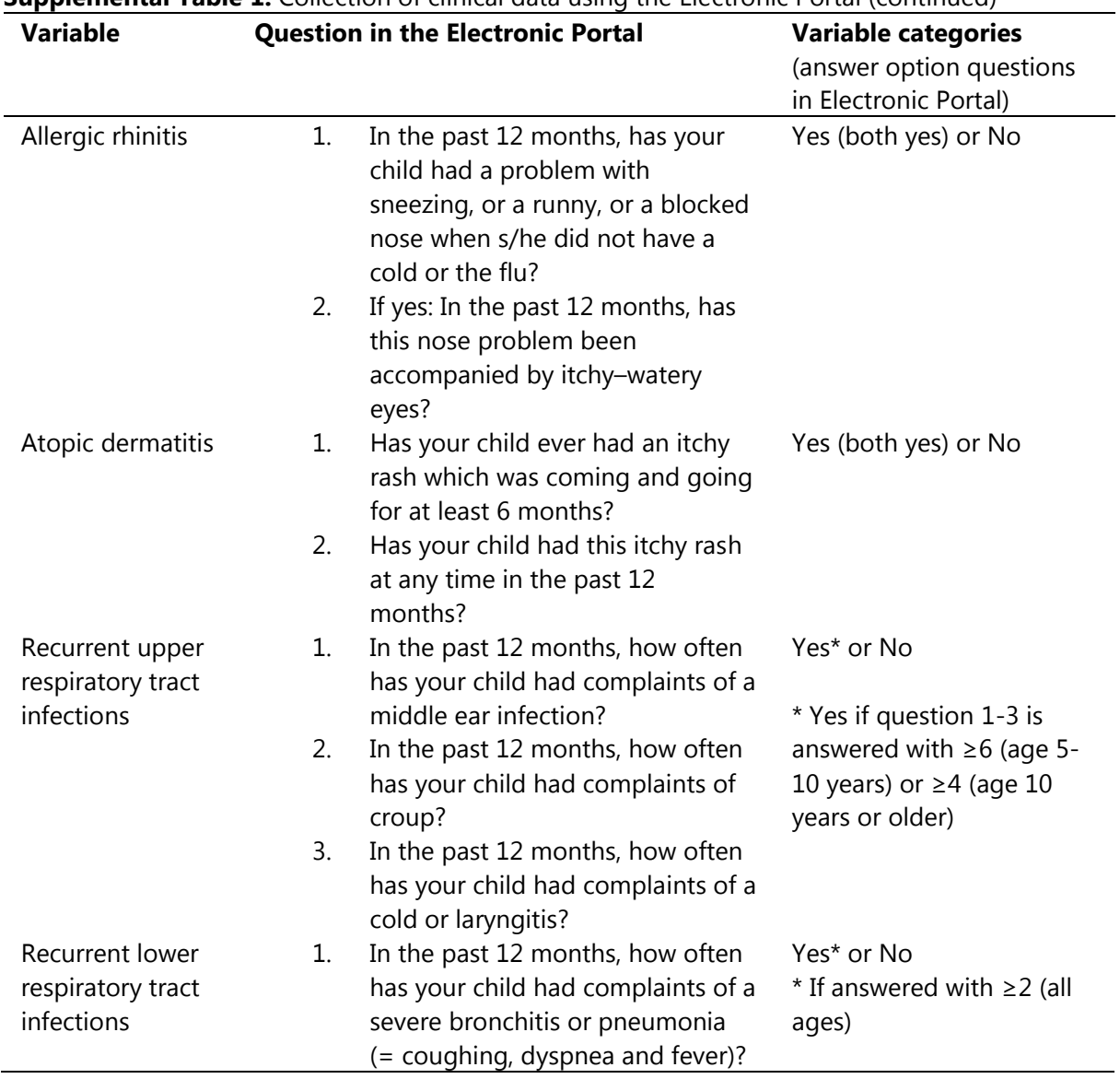


Supplemental Table 2. Full multivariable logistic regression analysis, with uncontrolled asthma (ACT score $<20$ ) as the outcome, in 408 children with asthma

\begin{tabular}{lll}
\hline & $\begin{array}{l}\text { Adjusted } \\
\text { OR (95\% CI) }\end{array}$ & P-value \\
\hline Age category & Ref & 0.739 \\
4 to 12 year & $1.09(0.66$ to 1.79$)$ & \\
12 to 18 year & $1.53(0.97$ to 2.42$)$ & 0.067 \\
$\begin{array}{l}\text { Male gender } \\
\text { Maternal education level }\end{array} \quad$ Ref & 0.110 \\
$\quad$ Low & $1.65(0.82$ to 3.40$)$ & \\
$\quad$ Middle & $1.02(0.51$ to 2.08$)$ & \\
$\quad$ High & $2.07(1.33$ to 3.25$)$ & 0.001 \\
Family history of asthma & $1.53(0.67$ to 3.51$)$ & 0.313 \\
Current smoke exposure & $1.43(0.91$ to 2.26$)$ & 0.123 \\
Current pet ownership & & 0.199 \\
Inhaled corticosteroids (daily) ${ }^{+}$ & Ref & \\
$\quad$ No & $1.09(0.63$ to 1.88$)$ & \\
Yes, adherent & $1.76(0.90$ to 3.45$)$ & \\
Yes, non-adherent & $0.75(0.41$ to 1.33$)$ & 0.320 \\
Food allergy & $0.94(0.58$ to 1.52$)$ & 0.856 \\
Allergic rhinitis & $1.04(0.66$ to 1.65$)$ & 0.798 \\
Atopic dermatitis & $2.40(1.51$ to 3.85$)$ & $<0.001$ \\
Recurrent upper RTIs (yes) & $1.99(1.23$ to 3.24$)$ & 0.005 \\
Recurrent lower RTIs (yes) & & 0.001 \\
Referral reason & $\operatorname{Ref}$ & \\
Atopic diseases other than asthma & $2.45(1.45$ to 4.18$)$ & \\
Asthma & & \\
\hline
\end{tabular}

${ }^{\dagger}$ Medication adherence was assessed by the Medication Adherence Rating Scale (MARS). Children with a total MARS score $\geq 21$ were considered adherent and a score $<21$ were considered non-adherent.

Abbreviations: ACT, Asthma Control Test; $C l$, confidence interval; $R T I s$, respiratory tract infections; OR, odds ratio; Ref, Reference categories. 
Prevalence and predictors of uncontrolled asthma 


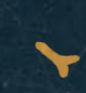

1

1

1

$y$

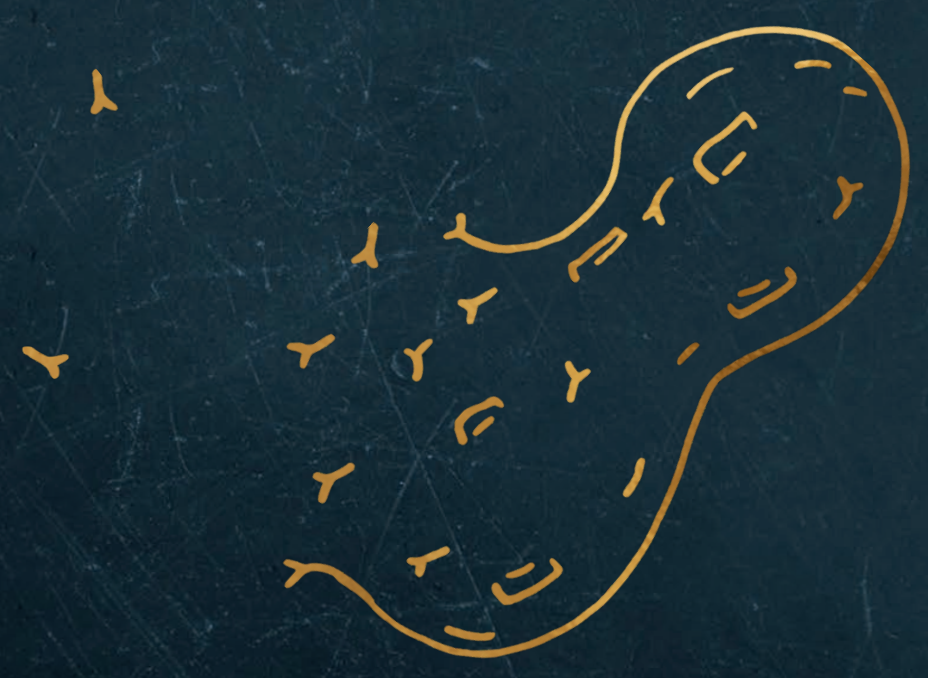




\section{Chapter 8}

\section{Perceived triggers of asthma impair quality of life in children with asthma}




\section{Abstract}

\section{Background}

Data on the impact of the number and nature of perceived asthma triggers on health-related quality of life (HRQL) in children are scarce.

\section{Objective}

To investigate the impact of perceived asthma triggers on both asthma-specific and generic HRQL in children.

\section{Methods}

A cross-sectional study was conducted among children (7 to 18 years) with asthma in secondary and tertiary care. Children were screened with electronic questionnaires regarding respiratory and allergic symptoms. Asthma-specific HRQL was assessed using the Pediatric Asthma Quality of Life Questionnaire (PAQLQ) (score range 1 to 7) and generic HRQL using the RAND questionnaire (score range 7 to 32). The Kruskall-Wallis test and one-way ANOVA was used to test the difference of respectively the PAQLQ and RAND score across the number of perceived asthma triggers $(0,1-2,3-4$, or $\geq 5)$. Univariable and multivariable linear regression analyses were performed to evaluate the association between individual triggers and HRQL.

\section{Results}

527 children with a mean (SD) age of 12.1 (2.9) years were included. Children with a higher number of perceived triggers had significantly lower PAQLQ and RAND scores (i.e. poorer HRQL). The difference in PAQLQ scores was clinically relevant between children with 0 versus $3-4$ or $\geq 5$ triggers and $1-2$ versus $\geq 5$ triggers (mean difference $0.66,1.02$ and 0.63 , respectively). Especially non-allergic triggers (physical exercise, the weather, (cigarette) smoke and emotions) were significantly associated with reduced PAQLQ scores. Emotions and food/drinks were associated with reduced RAND scores.

\section{Conclusion and Clinical relevance}

A higher number of perceived triggers of asthma was associated with reduced HRQL in children with asthma. Especially non-allergic triggers were associated with reduced $H R Q L$. 


\section{Introduction}

Asthma presents as one of the most common chronic inflammatory diseases in children in the developed world, affecting up to $9 \%$ of children. ${ }^{1}$ Asthma causes significant morbidity and has a negative impact on health-related quality of life (HRQL). ${ }^{2} \mathrm{HRQL}$ can be measured with asthma-specific and generic questionnaires. Asthma-specific HRQL reflect the perceived impact of asthma on a patient's life, while generic HRQL allow direct comparison between different diseases. Asthmaspecific HRQL is especially reduced in girls, in children with uncontrolled asthma and in those with frequent or severe asthma exacerbations. ${ }^{3,4}$ Children with asthma report poorer generic HRQL than children in the general population and children with allergic rhinitis, food allergy, atopic dermatitis and diabetes mellitus, but better generic HRQL than children with juvenile chronic arthritis. ${ }^{5,6}$

Asthma symptoms and exacerbations can be elicited by a range of allergic triggers (i.e. pollen, house dust or pets) and non-allergic triggers (i.e. physical exercise, respiratory tract infections, emotions or exposure to tobacco smoke). ${ }^{7}$ Only scarce data on the impact of perceived asthma triggers on HRQL in children are available. A higher number of perceived triggers of asthma can exert a toll on asthma-specific HRQL in children with severe or difficult-to-treat asthma. ${ }^{8,9}$ Data in children with mild or moderate asthma are lacking. Furthermore, it has been shown that asthmaspecific HRQL in children was associated with grass pollen exposure and airway inflammation ${ }^{10}$ and generic HRQL in children was reduced during pollen season. ${ }^{11}$ In adults, previous research has suggested that mainly non-allergic asthma triggers are associated with reduced asthma-specific HRQL. ${ }^{12}$

The aim of this study was to investigate the single and composite impact of perceived allergic and non-allergic triggers of asthma on asthma-specific and generic HRQL in children referred to secondary or tertiary care.

\section{Methods}

\section{Domain and data collection}

A cross-sectional study was performed among children referred with respiratory or allergic complaints to 8 secondary care centers and 1 tertiary care center in the Netherlands between June 2011 and August 2018. Eligible children were aged 7 to 18 years with parent-reported physician-diagnosed asthma. All children were invited for the Electronic Portal (EP) before an outpatient visit. The details of the EP have been previously published. ${ }^{13}$ In short, the EP is a web-based system with electronic questionnaires for children and their parents developed to collect information on asthma, allergic rhinitis, food allergy, atopic dermatitis and recurrent respiratory tract infections. This study was approved by the Medical 
Ethical Review Board of the University Medical Center in Utrecht, the Netherlands, number 10/348, and all parents and/or children gave informed consent before enrolment.

\section{Measurements}

Asthma-specific and generic HRQL

Asthma-specific HRQL in children was assessed using the child-reported Pediatric Asthma Quality of Life Questionnaire (PAQLQ). ${ }^{14}$ The PAQLQ is a validated instrument with 23 items in three domains: symptoms, activity limitation and emotional function. All PAQLQ-items are scored on a 7-point Likert response scale (i.e. $1=$ extremely bothered; $7=$ not bothered) with a 1 -week recall period. The final overall PAQLQ score is the mean score of all 23 items and domain-scores are the means of the items in the individual domains. The mean difference (MD) in PAQLQ scores between patients can be interpreted using the minimal clinically important differences (MCID). The MCID is the smallest change score that is considered clinically relevant. ${ }^{15}$ The MCID for the PAQLQ is 0.5 as estimated previously. ${ }^{16}$

Generic HRQL in children was assessed using the parent-reported RAND questionnaire. ${ }^{17}$ The RAND is a validated general health rating index for children with 7 questions that ask parents to assess the overall health status of their child with a 1-month recall period. The final RAND score is the summed score ranging from 7 to 32 with higher scores indicating better general health. The MCID of the RAND-questionnaire is $5.3 .^{18}$

\section{Perceived triggers of asthma}

Children ( $\geq 12$ years) or parents (children 7 to 12 years) were asked if, in the last 12 months, symptoms of wheezing in the chest were worsened after exposure to one or more of the following triggers: dust, pollen, pets, physical exercise, the weather, cold or the flu, (cigarette) smoke, emotions, food or drinks, detergent, or wool clothing. Dust, pollen and pets were categorized as allergic triggers; all other triggers were categorized as non-allergic triggers.

\section{Clinical data}

Information on patients' demographic and environmental characteristics was obtained using the electronic questionnaires in the EP. Asthma control during the last month was assessed using the Asthma Control Test (ACT). The previously defined cut-off was used to classify children as having well-controlled asthma (ACT score $\geq 20$ ) or uncontrolled asthma $(<20){ }^{19,20}$ Furthermore, the ISAAC core questionnaires for wheezing, allergic rhinitis, and atopic dermatitis were used. ${ }^{21}$ Comorbidities - i.e. allergic rhinitis, food allergy, atopic dermatitis and recurrent 
respiratory tract infections - were defined based on a combination of questions in the EP (Supplemental Table 1).

\section{Statistical analyses}

Children with complete HRQL data, information on perceived asthma triggers and clinical data used as confounders in the multivariable analysis were included in the analysis. Differences between children with complete and incomplete data were statistically evaluated by the chi-square test for categorical variables, the unpaired t-test for normally distributed continuous variables and the Mann-Whitney $U$ tests for non-normally distributed continuous variables with Bonferroni correction for multiple testing. Descriptive statistics were presented as number and percentages, means with standard deviations (SD) for normally distributed data and medians with interquartile ranges (IQR) for non-normally distributed data. The KruskallWallis test was used to compare the non-normally distributed PAQLQ score and one-way analysis of variance (ANOVA) was used to compare the normally distributed RAND score across the total number of perceived asthma triggers $(0,1$ $2,3-4$, or $\geq 5)$, the number of allergic triggers $(0,1,2$ or 3$)$ and the number of nonallergic triggers $(0,1-2,3-4$ or $\geq 5)$. Furthermore, the difference between the mean HRQL scores (mean difference, MD) of children in the different categories of triggers (e.g. 0 versus $1-2$ triggers) was calculated along with the $95 \%$ confidence interval $(95 \% \mathrm{CI})$ to interpret the change score using the minimal clinically important difference (MCID). In addition, the standardized mean difference (SMD) was calculated using Cohen's statistics to estimate the effect size. ${ }^{22}$ Based on Cohen's criteria, a SMD of 0.2 is considered small, 0.5 is moderate and $>0.8$ is large. $^{22}$

The association between the individual perceived asthma triggers and HRQL was estimated using univariable and multivariable linear regression analyses. The multivariable linear regression analyses was performed with all triggers and adjusted for age, sex, living environment, asthma control (well-controlled versus uncontrolled according to the Asthma Control Test) and the presence of allergic rhinitis, food allergy, atopic dermatitis or recurrent respiratory tract infections. The unadjusted and adjusted unstandardized regression coefficients for the difference in the outcome between patients with and without the trigger were estimated along with the $95 \%$ confidence interval and p-value. A p-value of less than 0.05 was considered statistically significant. All analyses were performed in SPSS Statistics for Windows (Version 22.0. Armonk, NY: IMB Corp). 


\section{Results}

\section{Patients' characteristics}

A total of 1869 children aged 7 to 18 years were included in the Electronic Portal in one of the participating secondary $(n=8)$ or tertiary care $(n=1)$ centers: 1743 children (93\%) completed the screening questionnaires, and the parents of 764 children (44\%) reported their child to have physician-diagnosed asthma. A total of 527 children (69\%) had complete data and were included in the analysis (Figure 1). Children included in the analysis were older compared to the children with incomplete data, but did not differ in the frequency of having any perceived trigger of asthma, the level of asthma control, asthma-specific health-related quality of life (HRQL) and generic HRQL (Supplemental Table 2).

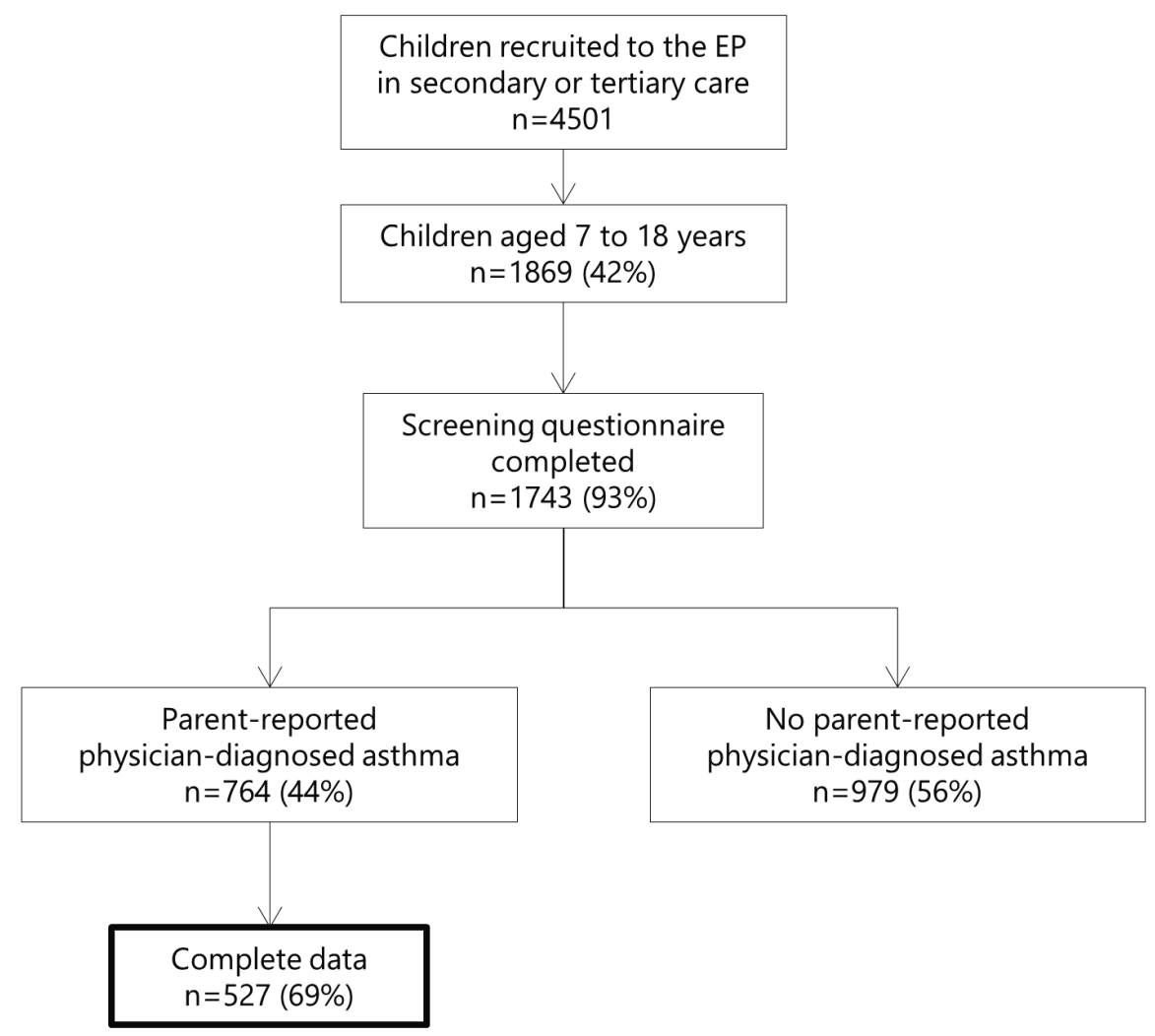

Figure 1. Study population flowchart

Abbreviations: EP, electronic portal; $n$, number. 
Patients' characteristics are presented in Table 1. Children had a mean (SD) age of 12.1 (2.9) years, 58\% were male, $88 \%$ were Dutch and $79 \%$ reported worsening of symptoms of wheezing in the chest after exposure to one or more asthma trigger in the past year. Half of the children (53\%) reported both perceived allergic and non-allergic asthma triggers. Figure 2 shows the proportion of individual triggers. The three most common perceived triggers of asthma were all non-allergic: physical exercise $(n=331 ; 63 \%)$, the weather $(n=269 ; 51 \%)$ and a cold or the flu $(n=268 ; 51 \%)$.

Table 1. Clinical characteristics of children with asthma

\begin{tabular}{|c|c|c|c|}
\hline & & n (\%) & $\begin{array}{l}\mathbf{n} \\
\text { available }\end{array}$ \\
\hline Age in years, mean (SD) & & $12.1(2.9)$ & 527 \\
\hline Male sex & & $304(58)$ & 527 \\
\hline Both parents Dutch & & $458(88)$ & 523 \\
\hline \multirow[t]{3}{*}{ Maternal education level } & Low & $68(13)$ & 524 \\
\hline & Middle & $212(41)$ & 524 \\
\hline & High & $244(47)$ & 524 \\
\hline Any asthma trigger & & $417(79)$ & 527 \\
\hline Both allergic and non-allergic asthma triggers & & $278(53)$ & 527 \\
\hline Only non-allergic asthma triggers & & $129(24)$ & 527 \\
\hline Only allergic asthma triggers & & $10(2)$ & 527 \\
\hline No asthma triggers & & $110(21)$ & 527 \\
\hline \multirow[t]{4}{*}{ Family history of } & Asthma & $239(45)$ & 526 \\
\hline & Allergic rhinitis & $353(67)$ & 525 \\
\hline & Atopic dermatitis & $268(51)$ & 525 \\
\hline & Food allergy & $53(14)$ & 379 \\
\hline \multirow[t]{2}{*}{ Current living environment } & Rural & $318(60)$ & 527 \\
\hline & Urban & $209(40)$ & 527 \\
\hline Pet ownership & & $234(44)$ & 527 \\
\hline Uncontrolled asthma $(\mathrm{ACT}<20)$ & & $200(38)$ & 527 \\
\hline Nocturnal wheezing/coughing in the past year & & $271(51)$ & 527 \\
\hline Daily inhaled corticosteroids & & $264(66)$ & 403 \\
\hline Inhaled short-acting $\beta 2$-agonists in the past week & & $271(51)$ & 527 \\
\hline \multirow[t]{4}{*}{ Comorbidities } & Allergic rhinitis & $296(56)$ & 527 \\
\hline & Atopic dermatitis & $215(41)$ & 527 \\
\hline & Food allergy & $153(29)$ & 527 \\
\hline & RRTI & $250(47)$ & 527 \\
\hline \multirow[t]{2}{*}{ Referred to } & Secondary care & $367(70)$ & 527 \\
\hline & Tertiary care & $160(30)$ & 527 \\
\hline \multirow[t]{6}{*}{ Referral reason } & Asthma & $209(50)$ & 415 \\
\hline & Food allergy & $124(30)$ & 415 \\
\hline & Allergic rhinitis & $32(8)$ & 415 \\
\hline & Atopic dermatitis & $32(8)$ & 415 \\
\hline & RRTI & $10(2)$ & 415 \\
\hline & Other & $19(5)$ & 415 \\
\hline
\end{tabular}

Abbreviations: ACT, Asthma Control Test; $n$, number; RRTI, recurrent respiratory tract infections; $S D$, standard deviation. 


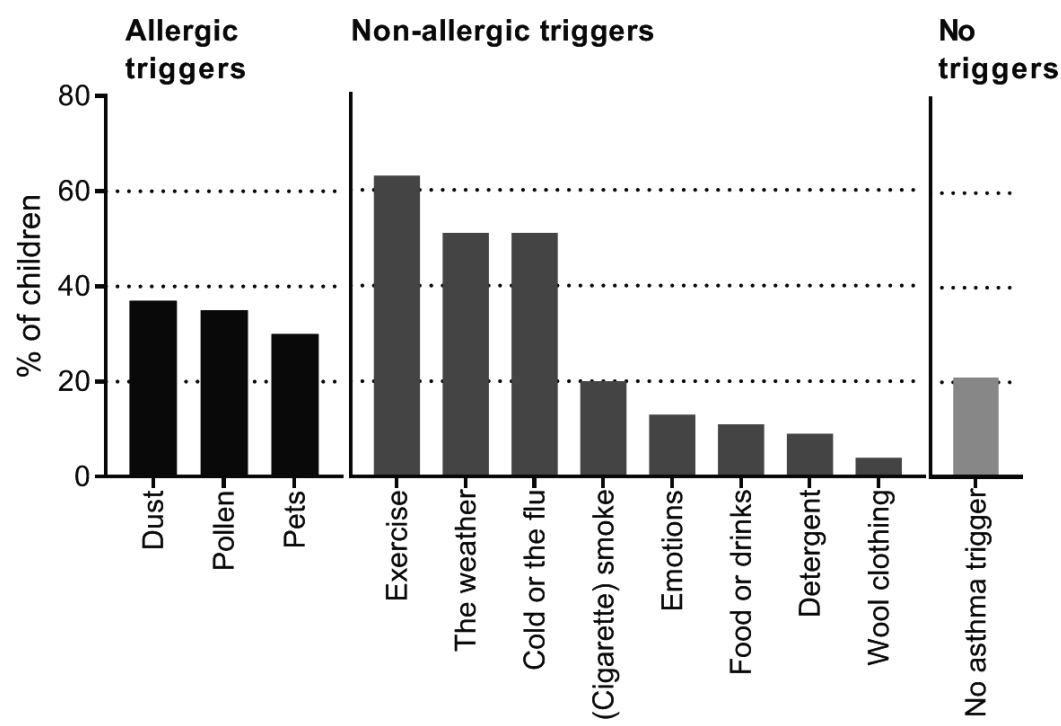

Figure 2. Proportion of asthma triggers in children with asthma $(n=527)$

The association between asthma triggers and asthma-specific HRQL

A higher number of perceived triggers of asthma was associated with reduced asthma-specific HRQL (Figure 3a). Children with a higher number of triggers had significantly lower PAQLQ scores (Table 2). The same association was observed for the domain-specific PAQLQ scores and after stratification by allergic and nonallergic triggers.

The difference of the overall PAQLQ score was considered clinically relevant between children with 0 and 3-4 triggers and 0 and $\geq 5$ triggers (mean difference [MD] 0.66 and 1.02, respectively ) with respectively moderate and large effect size (standardized mean difference [SMD] 0.74 and 1.10). Furthermore, the difference was clinically relevant between children with $1-2$ and $\geq 5$ triggers (MD 0.63) with moderate effect size (SMD 0.65) (Supplemental Table 3). In addition, clinically relevant differences of the domain-specific PAQLQ scores were observed (Supplemental Table 3). Children with only non-allergic asthma triggers had a significantly lower median (IQR) PAQLQ score (i.e. worse HRQL) (6.1 (5.2-6.7); $\mathrm{n}=129)$ compared to children with only allergic asthma triggers $(6.7(6.1-6.9) ; n=10)$ $(p=0.029)$.

\section{The association between asthma triggers and generic HRQL}

A higher number of perceived triggers of asthma was associated with reduced generic HRQL as assessed with the RAND questionnaire (Figure 3b). Children with a higher number of triggers had significantly lower RAND scores (Table 2). The same association was observed if the analysis was stratified by allergic and non-allergic triggers. The difference in RAND scores were not considered clinically relevant as 
the minimal clinically important difference was not exceeded. The effect size was moderate between children with 0 and $\geq 5$ triggers (SMD 0.68) and between children with 1-2 and $\geq 5$ triggers (SMD 0.62) (Supplemental Table 3).

The RAND score was comparable between children with only non-allergic asthma triggers (mean (SD) 21.1 (4.4)) and children with only allergic asthma triggers (22.1 (3); $n=10)(p=0.494)$.

Table 2. The number of asthma triggers and asthma-specific (PAQLQ) and generic (RAND) quality of life scores

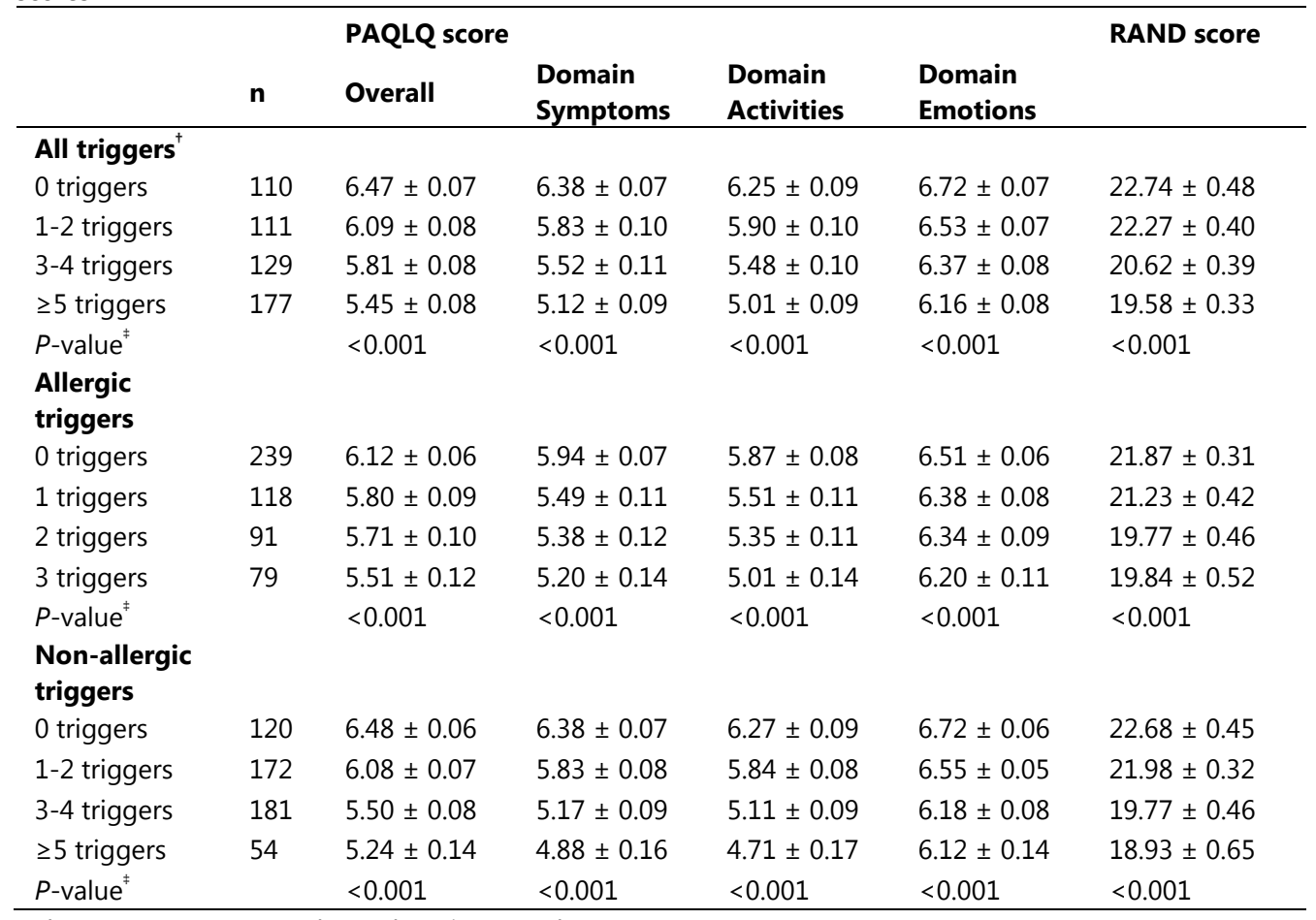

Values are mean $\pm \mathrm{SE}$, unless otherwise stated.

${ }^{+}$All triggers included allergic triggers (dust, pollen, pets) and non-allergic asthma triggers (physical exercise, the weather, cold or the flu, (cigarette) smoke, emotions, food or drinks, detergent, wool clothing).

${ }^{\ddagger}$ Statistical comparisons between trigger categories using Kruskall-Wallis test for the PAQLQ scores and one-way ANOVA for the RAND score with $P$-values of less than 0.004 taken to be significant according to Bonferroni correction for multiple testing.

Abbreviations: n, number; PAQLQ, Pediatric Asthma Quality of Life Questionnaire; RAND, RAND generalhealth rating index; $S E$, standard error. 


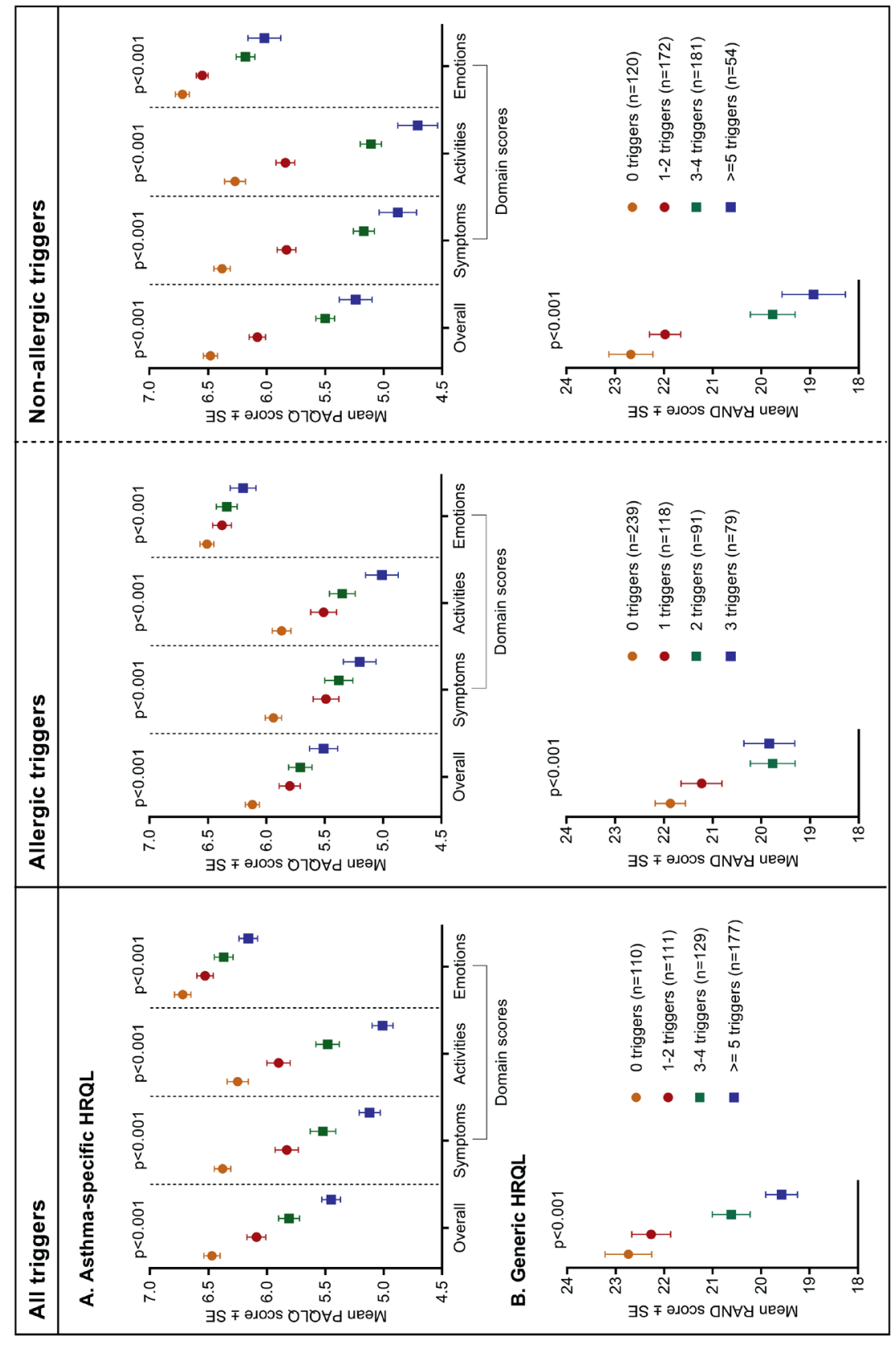

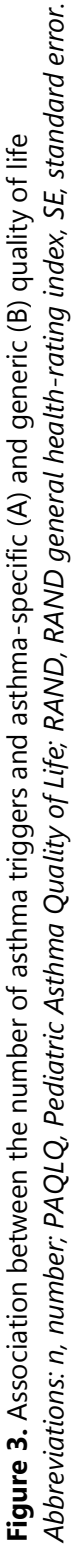




\section{Single and composite effects of asthma triggers on asthma-specific HRQL}

In univariable linear regression analysis, all allergic asthma triggers were associated with a lower overall PAQLQ score (i.e. poorer asthma-specific HRQL) (Table 3a). In addition, all non-allergic asthma triggers except wool clothing were associated with a lower overall PAQLQ score. In the adjusted multivariable analysis, physical exercise, the weather, (cigarette) smoke and emotions were independently associated with a lower overall PAQLQ score. None of the perceived allergic triggers was independently associated with the overall PAQLQ score.

The multivariable analysis was repeated with the domain-specific PAQLQ scores as the outcome (Supplemental Table 4). Physical exercise and (cigarette) smoke were independently associated with a lower symptom domain score. Pets, physical exercise, the weather and (cigarette) smoke were independently associated with a lower activity limitations domain score. The asthma trigger emotions was independently associated with a lower emotional function domain score.

\section{Single and composite effects of asthma triggers on generic HRQL}

In univariable linear regression analysis, all allergic asthma triggers were associated with a lower RAND score (i.e. poorer generic HRQL) (Table 3b). Again, all nonallergic asthma triggers except wool clothing were associated with a lower RAND score.

In the adjusted multivariable analysis, only emotions and food or drinks were independently associated with a lower RAND score. None of the perceived allergic triggers was independently associated with the RAND score. 
Table 3. Linear regression analysis for asthma-specific (a) and generic (b) quality of life

\begin{tabular}{|c|c|c|c|c|c|c|}
\hline \multicolumn{7}{|c|}{ a. Overall PAQLQ score } \\
\hline & \multicolumn{2}{|c|}{ Univariable analysis } & \multicolumn{4}{|c|}{ Multivariable analysis } \\
\hline & $\beta$ & $95 \% \mathrm{CI}$ & $P$-value & $a \beta^{+}$ & $95 \% \mathrm{CI}$ & $P$-value \\
\hline \multicolumn{7}{|l|}{ Allergic triggers } \\
\hline Dust & -0.51 & -0.68 to -0.34 & $<0.001$ & -0.09 & -0.25 to 0.08 & 0.293 \\
\hline Pollen & -0.27 & -0.45 to -0.08 & 0.004 & 0.15 & -0.03 to 0.33 & 0.095 \\
\hline Pets & -0.35 & -0.53 to -0.16 & $<0.001$ & -0.03 & -0.19 to 0.14 & 0.760 \\
\hline \multicolumn{7}{|c|}{ Non-allergic triggers } \\
\hline Physical exercise & -0.70 & -0.87 to -0.53 & $<0.001$ & -0.16 & -0.32 to 0.00 & 0.047 \\
\hline The weather & -0.66 & -0.83 to -0.50 & $<0.001$ & -0.17 & -0.33 to -0.01 & 0.038 \\
\hline Cold or the flu & -0.55 & -0.71 to -0.38 & $<0.001$ & 0.00 & -0.16 to 0.15 & 0.954 \\
\hline (Cigarette) smoke & -0.75 & -0.96 to -0.55 & $<0.001$ & -0.27 & -0.47 to -0.08 & 0.006 \\
\hline Emotions & -0.75 & -1.00 to -0.50 & $<0.001$ & -0.28 & -0.49 to -0.08 & 0.007 \\
\hline Food or drinks & -0.29 & -0.57 to -0.02 & 0.037 & -0.14 & -0.36 to 0.08 & 0.201 \\
\hline Detergent & -0.68 & -0.97 to -0.38 & $<0.001$ & 0.05 & -0.20 to 0.31 & 0.670 \\
\hline Wool clothing & -0.31 & -0.73 to 0.11 & 0.153 & 0.22 & -0.10 to 0.55 & 0.181 \\
\hline
\end{tabular}

\section{b. RAND score}

\begin{tabular}{|c|c|c|c|c|c|c|}
\hline & \multicolumn{2}{|c|}{ Univariable analysis } & \multicolumn{4}{|c|}{ Multivariable analysis } \\
\hline & $\beta$ & $95 \% \mathrm{CI}$ & $P$-value & $a \beta^{+}$ & $95 \% \mathrm{CI}$ & $P$-value \\
\hline \multicolumn{7}{|l|}{ Allergic triggers } \\
\hline Dust & -2.07 & -2.89 to -1.26 & $<0.001$ & -0.65 & -1.55 to 0.26 & 0.162 \\
\hline Pollen & -1.24 & -2.08 to -0.40 & 0.004 & 0.27 & -0.71 to 1.25 & 0.594 \\
\hline Pets & -0.92 & -1.79 to -0.05 & 0.039 & 0.20 & -0.74 to 1.14 & 0.676 \\
\hline \multicolumn{7}{|c|}{ Non-allergic triggers } \\
\hline Physical exercise & -1.46 & -2.28 to -0.63 & 0.001 & 0.46 & -0.41 to 1.34 & 0.299 \\
\hline The weather & -1.88 & -2.67 to -1.09 & $<0.001$ & -0.17 & -1.06 to 0.73 & 0.717 \\
\hline Cold or the flu & -2.21 & -2.99 to -1.42 & $<0.001$ & -0.55 & -1.41 to 0.31 & 0.212 \\
\hline (Cigarette) smoke & -1.79 & -2.78 to -0.79 & $<0.001$ & 0.00 & -1.08 to 1.08 & 0.998 \\
\hline Emotions & -2.75 & -3.94 to -1.56 & $<0.001$ & -1.49 & -2.64 to -0.35 & 0.011 \\
\hline Food or drinks & -2.24 & -3.51 to -0.96 & 0.001 & -1.86 & -3.09 to -0.63 & 0.003 \\
\hline Detergent & -2.10 & -3.49 to -0.72 & 0.003 & -0.06 & -1.47 to 1.35 & 0.930 \\
\hline Wool clothing & -1.02 & -2.99 to 0.96 & 0.312 & 0.85 & -0.98 to 2.67 & 0.362 \\
\hline
\end{tabular}

A negative $\beta$-value indicates a negative impact on $\mathrm{HRQL}$-scores (i.e. poorer $\mathrm{HRQL}$ ).

${ }^{+}$Adjusted for age, gender, living environment, asthma control, allergic rhinitis, food allergy, atopic dermatitis and recurrent respiratory tract infections.

Abbreviations: (a) $\beta$, (adjusted) unstandardized regression coefficient; $\mathrm{Cl}$, confidence interval; PAQLQ, Pediatric Asthma Quality of Life Questionnaire; RAND, RAND general-health rating index.

\section{Discussion}

Our cross-sectional study demonstrated that amongst a broad population of children with asthma, a higher number of perceived allergic and non-allergic asthma triggers was strongly associated with reduced asthma-specific as well as generic health-related quality of life (HRQL). The majority of children (79\%) reported one or more perceived trigger(s) of asthma. Non-allergic asthma triggers were independently associated with reduced HRQL: physical exercise, the weather, 
(cigarette) smoke and emotions with asthma-specific HRQL, and emotions and food/drinks with generic HRQL.

Our study is the first to indicate that not only asthma-specific HRQL was reduced in children with a higher number of asthma triggers but also generic HRQL was reduced. Previous research showed an association between a higher number of perceived triggers of asthma and reduced asthma-specific HRQL in children and adolescents with severe or difficult-to-treat asthma ${ }^{8,9}$, and in children participating in an asthma education program with symptom perception interventions. ${ }^{23} \mathrm{We}$ were able to confirm these findings in children and adolescents with asthma of varying severity in secondary or tertiary care. The impact of asthma triggers on HRQL may be explained by the fact that asthma triggers are associated with greater severity and frequency of asthma exacerbations, emergency treatments, health care visits and hospitalization. ${ }^{12,24}$ In addition, a higher number of asthma triggers may result in an increased perceived burden of asthma. Furthermore, children with a higher number of asthma triggers may try to avoid asthma triggers which may have an additional negative impact on asthma-specific and generic HRQL.

Our study suggests that especially non-allergic triggers were independently associated with HRQL. It can be hypothesized that triggers that are difficult to control, i.e. the weather, (cigarette) smoke and emotions, exert a greater influence on $\mathrm{HRQL}$ than triggers that are more easily controlled or treated. Allergic triggers, i.e. pets, dust or pollen, might exert less influence on HRQL because adequate treatment is available to reduce the symptoms caused by these triggers However, we might not have detected an association between allergic triggers and HRQL as data on allergen sensitization were not available. Interestingly, the asthma trigger emotions was consistently associated with both asthma-specific and generic HRQL in our study population. Strong emotions such as fear, excitement, stress, anger or laughter may cause asthma symptoms due to an abnormal breathing pattern. Furthermore, the asthma trigger emotions might indicate the presence of psychiatric comorbidities, e.g. depression and anxiety. Psychiatric comorbidities are known to be more prevalent among patients with asthma and are associated with worse asthma control, increased asthma exacerbations and emergency visits. ${ }^{7}$

Our results highlight the importance of trigger identification in children with asthma. Physicians should pay attention to the identification of asthma triggers and, in turn, instruct children and parents regarding avoidance strategies or treatment of the identified triggers. For instance, the impact of the trigger physical exercise on HRQL might be reduced when children are advised to use a shortacting $\beta 2$-agonist before exercising ${ }^{7}$ and the impact of the trigger emotions might be reduced when children seek psychological support to reduce emotional stress. Although allergic triggers were not independently associated with HRQL in our 
analysis, we showed that a higher cumulative number of allergic and non-allergic asthma triggers was associated with reduced asthma-specific and generic HRQL. Therefore, specific instructions directed at children with allergic triggers might be beneficial as well. For instance, the impact of the trigger dust on HRQL might be reduced when instructions are given on house cleaning and the impact of the trigger pollen might be reduced when adequate (pharmacological) treatment is initiated.

The major limitation of the present study is that the asthma diagnosis and asthma triggers were self-reported by parents. Furthermore, data on allergen sensitization were not available. However, patient-reported data do provide meaningful insight into the perception of allergic and non-allergic asthma triggers and HRQL among parents and children. Additionally, asthma-specific and generic HRQL cannot be directly compared as asthma-specific HRQL was assessed in children and generic HRQL in parents. Previous research reported a lack of agreement between children's self-reported and parent proxy-reported HRQL and suggested to include both perspectives in HRQL research as both are complementary to each other. ${ }^{25,26}$. In addition, we were not able to assess asthma severity as we did not collect data on the frequency of asthma exacerbations, emergency department visits and hospitalizations. Finally, the cross-sectional design of this study limits the assessment of possible long-term effects. Strengths of this study are the large sample size, reliable data collection in routine secondary and tertiary care using validated questionnaires in children and parents and the assessment of both asthma-specific and generic HRQL.

In summary, we demonstrated that a higher number of perceived allergic and nonallergic triggers of asthma was strongly associated with reduced asthma-specific and generic health-related quality of life (HRQL) in children with asthma. Especially non-allergic asthma triggers were independently associated with reduced HRQL. Accurate identification and, in turn, avoidance and/or treatment of triggers in children with asthma may improve health-related quality of life.

\section{Acknowledgments}

The authors would like to thank the members of the Expert Network: Monique Gorissen, Bart van Ewijk, Walter Balemans, Esther de Vries, Maartje van Velzen, Frank Eskes, Gordon Slabbers and René van Gent and participant of the Electronic Portal: Marike Stadermann for their collaboration and work within their centres. Furthermore, the authors would like to thank Paco Welsing for his statistical advice. 


\section{References}

1. National Health Interview Survey (NHIS) Data. Center for Disease Control and Prevention. https://www.cdc.gov/asthma/most_recent_data.htm. Accessed July 31, 2018.

2. Chen $\mathrm{H}$, Gould MK, Blanc PD, et al. Asthma control, severity, and quality of life: Quantifying the effect of uncontrolled disease. J Allergy Clin Immunol. 2007;120(2):396-402.

3. Stridsman C, Backman H, Eklund BM, Rönmark E, Hedman L. Adolescent girls with asthma have worse asthma control and health-related quality of life than boys-A population based study. Pediatr Pulmonol. 2017;52(7):866-872.

4. Jonsson M, Bergström A, Egmar AC, Hedlin G, Lind T, Kull I. Asthma during adolescence impairs health-related quality of life. J Allergy Clin Immunol Pract. 2016;4(1):144-146.e2.

5. Covaciu C, Bergström A, Lind T, Svartengren M, Kull I. Childhood allergies affect health-related quality of life. J Asthma. 2013;50(5):522-528.

6. Norrby U, Nordholm L, Andersson-Gäre B, Fasth A. Health-related quality of life in children diagnosed with asthma, diabetes, juvenile chronic arthritis or short stature. Acta Paediatr Int $J$ Paediatr. 2006;95(4):450-456.

7. Global Initiative for Asthma (GINA). Global Strategy for Asthma Management and Prevention. Available from: https://ginasthma.org (2018). Accessed Aug 8, 2018.

8. Luskin AT, Chipps BE, Rasouliyan L, Miller DP, Haselkorn T, Dorenbaum A. Impact of Asthma Exacerbations and Asthma Triggers on Asthma-related Quality of Life in Patients with Severe or Difficult-to-Treat Asthma. J Allergy Clin Immunol Pract. 2014;2(5):544-552.

9. Chipps BE, Haselkorn T, Rosén K, Mink DR, Trzaskoma BL, Luskin AT. Asthma Exacerbations and Triggers in Children in TENOR: Impact on Quality of Life. J Allergy Clin Immunol Pract. 2018;6(1):169-176.e2.

10. Roberts G, Mylonopoulou M, Hurley C, Lack G. Impairment in quality of life is directly related to the level of allergen exposure and allergic airway inflammation. Clin Exp Allergy. 2005;35(10):1295-1300.

11. Kiotseridis $\mathrm{H}$, Cilio $\mathrm{CM}$, Bjermer $\mathrm{L}$, et al. Quality of life in children and adolescents with respiratory allergy, assessed with a generic and disease-specific instrument. Clin Respir J. 2013;7(2):168-175.

12. Ritz T, Wittchen HU, Klotsche J, Mühlig S, Riedel O. Asthma trigger reports are associated with low quality of life, exacerbations, and emergency treatments. Ann Am Thorac Soc. 2016;13(2):204-211.

13. Zomer-Kooijker K, van Erp FC, Balemans WAF, van Ewijk BE, van der Ent CK. The expert network and electronic portal for children with respiratory and allergic symptoms: Rationale and design. BMC Pediatr. 2013;13(1):9.

14. Raat H, Bueving HJ, De Jongste JC, Grol MH, Juniper EF, Van Der Wouden JC. Responsiveness, longitudinal- and cross-sectional construct validity of the Pediatric Asthma Quality of Life Questionnaire (PAQLQ) in Dutch children with asthma. Qual Life Res. 2005;14(1):265-272.

15. Revicki $D$, Hays RD, Cella D, Sloan J. Recommended methods for determining responsiveness and minimally important differences for patient-reported outcomes. J Clin Epidemiol. 2008;61(2):102-109.

16. Juniper EF, Guyatt GH, Willan A, Griffith LE. Determining a minimal important change in a disease-specific quality of life questionnaire. J Clin Epidemiol. 1994;47(1):81-87.

17. Post M, Kuyenhoven M, Verheij M, de Melker R, Hoes A. The Dutch "Rand General Health Rating Index for Children": a questionnaire measuring the general health status of children. Ned 
Tijdschr Geneeskd. 1998;142(49):2680-2683.

18. Brouwer CNM, Schilder AGM, Van Stel HF, et al. Reliability and validity of functional health status and health-related quality of life questionnaires in children with recurrent acute otitis media. Qual Life Res. 2007;16(8):1357-1373.

19. Nathan RA, Sorkness CA, Kosinski M, et al. Development of the Asthma Control Test: A survey for assessing asthma control. J Allergy Clin Immunol. 2004;113:59-65.

20. Liu AH, Zeiger $\mathrm{R}$, Sorkness $\mathrm{C}$, et al. Development and cross-sectional validation of the Childhood Asthma Control Test. J Allergy Clin Immunol. 2007;119:817-825.

21. Asher MI, Keil U, Anderson HR, et al. International study of asthma and allergies in childhood (ISAAC): Rationale and methods. Eur Respir J. 1995;8(3):483-491.

22. Cohen J. Statistical Power Analysis for the Behavioral Sciences. New York: Academic Press; 1977.

23. Janssens $T$, Harver A. Effects of Symptom Perception Interventions on Trigger Identification and Quality of Life in Children with Asthma. Pulm Med. 2015;2015:825137.

24. Gautier C, Charpin D. Environmental triggers and avoidance in the management of asthma. J Asthma Allergy. 2017;10:47-56.

25. Silva CM, Barros L, Simões F. Health-related quality of life in paediatric asthma: Childrens and parents perspectives. Psychol Heal Med. 2015;20(8):940-954.

26. Eiser C, Morse R. Can Parents Rate Their Child 's Health-Related Quality of Life? Results of a Systematic Review. Qual Life Res. 2001;10(4):347-357. 


\section{Supplemental tables}

Supplemental Table 1. Collection of clinical data using the Electronic Portal

\begin{tabular}{|c|c|c|}
\hline Variable & Question in the Electronic Portal & $\begin{array}{l}\text { Variable categories } \\
\text { (answer option questions in } \\
\text { Electronic Portal) }\end{array}$ \\
\hline $\begin{array}{l}\text { Physician-diagnosed } \\
\text { asthma }\end{array}$ & $\begin{array}{l}\text { Has your child been diagnosed with } \\
\text { asthma? } \\
\text { a. Yes, by a general practitioner } \\
\text { b. Yes, by a pediatrician } \\
\text { c. Yes, by a pulmonologist } \\
\text { d. No }\end{array}$ & Yes $(a, b$ or $c)$ or No $(d)$ \\
\hline Both parents Dutch & $\begin{array}{l}\text { What ethnic group do you belong to? } \\
\text { Mother/Father (separate questions) } \\
\text { a. Dutch } \\
\text { b. Turkish } \\
\text { c. Moroccan } \\
\text { d. Surinamese } \\
\text { e. Antillean } \\
\text { f. Other }\end{array}$ & Yes (both parents a) or No \\
\hline $\begin{array}{l}\text { Maternal education } \\
\text { level }\end{array}$ & $\begin{array}{l}\text { What is the highest level of education } \\
\text { you have completed? } \\
\text { a. Elementary school } \\
\text { b. Lower general secondary } \\
\text { c. Intermediate vocational } \\
\text { d. Higher general secondary } \\
\text { e. Higher vocational education } \\
\text { f. University }\end{array}$ & $\begin{array}{l}\text { Low }(a-b) \text {, Middle (c-d) or } \\
\text { High }(e-f)\end{array}$ \\
\hline $\begin{array}{l}\text { Family history of } \\
\text { asthma, allergic } \\
\text { rhinitis, food allergy } \\
\text { or atopic dermatitis }\end{array}$ & $\begin{array}{l}\text { Do one or more family members suffer } \\
\text { from asthma, allergic rhinitis, food } \\
\text { allergy or atopic dermatitis? (four } \\
\text { separate questions) } \\
\text { a. No } \\
\text { b. Yes, the father of the child } \\
\text { c. Yes, the mother of the child } \\
\text { d. Yes, brother(s) of the child } \\
\text { e. Yes, sister(s) of the child }\end{array}$ & Yes (b and/or $c$ ) or No \\
\hline $\begin{array}{l}\text { Current living } \\
\text { environment }\end{array}$ & $\begin{array}{l}\text { Can you describe your living } \\
\text { environment? } \\
\begin{array}{l}\text { a. A small town } \\
\text { b. Rural } \\
\text { c. A city center } \\
\text { d. Urban }\end{array}\end{array}$ & Rural $(a-b)$ or Urban $(c-d)$ \\
\hline
\end{tabular}


Chapter 8

Supplemental Table 1. Collection of clinical data using the Electronic Portal (continued)

\begin{tabular}{|c|c|c|}
\hline Variable & Question in the Electronic Portal & $\begin{array}{l}\text { Variable categories } \\
\text { (answer option questions in } \\
\text { Electronic Portal) }\end{array}$ \\
\hline $\begin{array}{l}\text { Nocturnal } \\
\text { wheezing/coughing } \\
\text { in the past year }\end{array}$ & $\begin{array}{l}\text { 1. In the past } 12 \text { months, how often, on } \\
\text { average, has your child's sleep been } \\
\text { disturbed due to wheezing? } \\
\text { a. Never woken by wheezing } \\
\text { b. Less than one night per week } \\
\text { c. One or more nights per week } \\
\text { 2. In the past } 12 \text { months, has your child } \\
\text { had a dry cough at night, apart from a } \\
\text { cough associated with a cold or a chest } \\
\text { infection? }\end{array}$ & $\begin{array}{l}\text { Yes* or No } \\
\text { * Yes if question } 1 \text { is answered } \\
\text { b or c and/or question } 2 \\
\text { answered 'yes' }\end{array}$ \\
\hline $\begin{array}{l}\text { Inhaled short-acting } \\
\beta 2 \text {-agonists in the } \\
\text { past week }\end{array}$ & $\begin{array}{l}\text { In the past week, how often has your } \\
\text { child inhaled a short-acting } \\
\text { bronchodilator (e.g. Ventolin, } \\
\text { Salbutamol, Bricanyl) on most days? } \\
\text { a. Never } \\
\text { b. } 1-2 \text { inhalations on most days } \\
\text { c. } 3-4 \text { inhalations on most days } \\
\text { d. } 5-8 \text { inhalations on most days } \\
\text { e. } 9-12 \text { inhalations on most days } \\
\text { f. } 13-16 \text { inhalations on most } \\
\text { g. days } \\
\text { g. } 16 \text { inhalations on most days }\end{array}$ & Yes $(b-g)$ or No (a) \\
\hline $\begin{array}{l}\text { Daily inhaled } \\
\text { corticosteroids }\end{array}$ & $\begin{array}{l}\text { Has your child used daily inhaled } \\
\text { corticosteroids (e.g. Pulmicort, Flixotide, } \\
\text { Seretide, Qvar)? } \\
\text { a. Yes } \\
\text { b. No }\end{array}$ & Yes (a) or No (b) \\
\hline Allergic rhinitis & $\begin{array}{l}\text { 1. In the past } 12 \text { months, has your child } \\
\text { had a problem with sneezing, or a runny, } \\
\text { or a blocked nose when s/he did not } \\
\text { have a cold or the flu?" } \\
\text { 2. If question } 1 \text { is answered "yes": "In the } \\
\text { past } 12 \text { months, has this nose problem } \\
\text { been accompanied by itchy-watery } \\
\text { eyes?" }\end{array}$ & $\begin{array}{l}\text { Yes (both answered 'yes') or } \\
\text { No }\end{array}$ \\
\hline
\end{tabular}


Supplemental Table 1. Collection of clinical data using the Electronic Portal (continued)

\begin{tabular}{|c|c|c|}
\hline Variable & Question in the Electronic Portal & $\begin{array}{l}\text { Variable categories } \\
\text { (answer option questions in } \\
\text { Electronic Portal) }\end{array}$ \\
\hline Food allergy & $\begin{array}{l}\text { 1. Has your child ever had a food } \\
\text { allergy? } \\
\text { 2. Within what time period after food } \\
\text { ingestion did your child develop } \\
\text { symptoms? } \\
\text { 3. What symptoms did your child } \\
\text { experience after food ingestion? }\end{array}$ & $\begin{array}{l}\text { Yes* or No } \\
\text { * Yes if question } 1 \text { is answered } \\
\text { 'yes', and symptoms occurred } \\
\text { within two hours after } \\
\text { ingestion of the food (question } \\
\text { 2) and food allergic symptoms } \\
\text { were relevant (question 3): } \\
\text { urticaria, oral allergy, } \\
\text { angioedema, upper or lower } \\
\text { respiratory complaints, } \\
\text { vomiting, loss of } \\
\text { consciousness or anaphylaxis. }\end{array}$ \\
\hline Atopic dermatitis & $\begin{array}{l}\text { 1. Has your child ever had an itchy rash } \\
\text { which was coming and going for at least } \\
6 \text { months? } \\
\text { 2. Has your child had this itchy rash at } \\
\text { any time in the past } 12 \text { months? }\end{array}$ & Yes (both answered 'yes') or No \\
\hline $\begin{array}{l}\text { Recurrent } \\
\text { respiratory tract } \\
\text { infections in the past } \\
\text { year }\end{array}$ & $\begin{array}{l}\text { 1. In the past } 12 \text { months, how often has } \\
\text { your child had complaints of a middle } \\
\text { ear infection? } \\
\text { 2. In the past } 12 \text { months, how often has } \\
\text { your child had complaints of croup? } \\
\text { 3. In the past } 12 \text { months, how often has } \\
\text { your child had complaints of a cold or } \\
\text { laryngitis? } \\
\text { 4. In the past } 12 \text { months, how often has } \\
\text { your child had complaints of a severe } \\
\text { bronchitis or pneumonia (= coughing, } \\
\text { dyspnea and fever)? }\end{array}$ & $\begin{array}{l}{ }^{*} \text { Yes if question } 1 \text { through } 3 \text { is } \\
\text { answered } \geq 6 \text { (age } 5 \text { to } 10 \\
\text { years) or } \geq 4 \text { (age } 10 \text { years or } \\
\text { older) and/or question } 4 \text { is } \\
\text { answered } \geq 2 \text { (all ages). }\end{array}$ \\
\hline
\end{tabular}

The questions were translated from Dutch into English.

ISAAC core questionnaires for wheezing

ISAAC core questionnaires for allergic rhinitis

"ISAAC core questionnaires for atopic dermatitis 
Supplemental Table 2. Differences between complete and incomplete cases

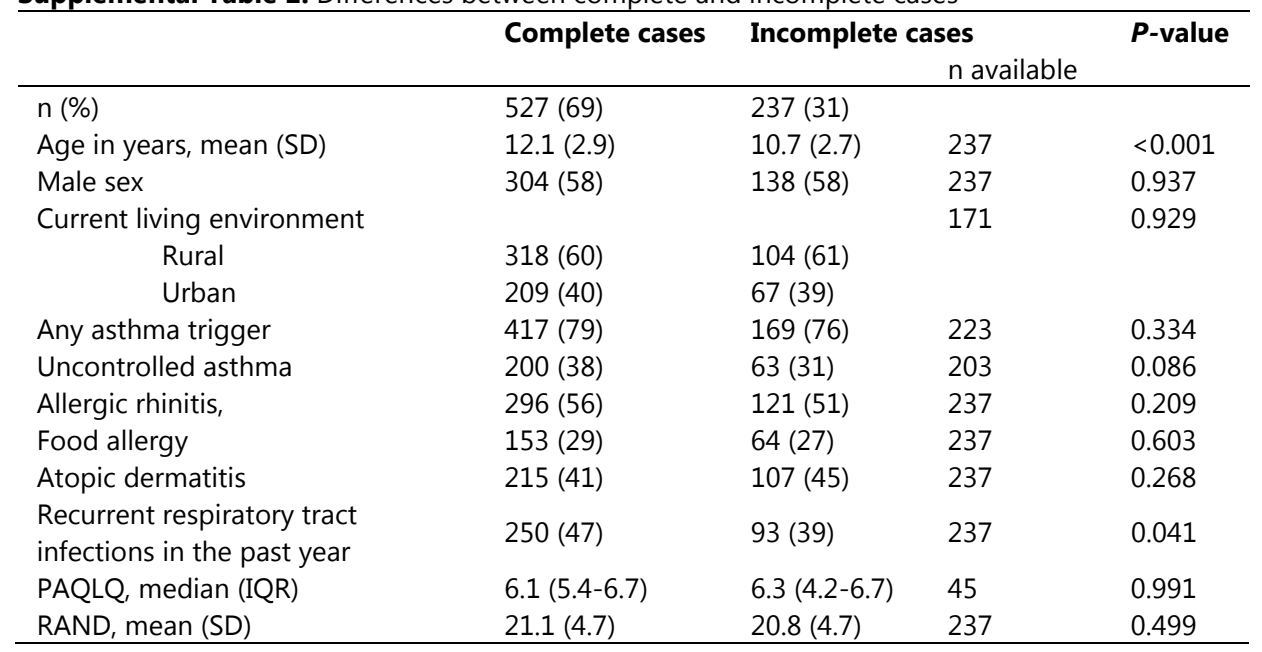

Values are numbers (\%), unless otherwise indicated.

Abbreviations: IQR, interquartile range; $n$, number; PAQLQ, Pediatric Asthma Quality of Life Questionnaire; $R A N D, R A N D$ general-health rating index; $S D$, standard deviation. 
Supplemental Table 3. Differences in asthma-specific (a) and generic (b) quality of life among children within different categories of asthma triggers

\begin{tabular}{|c|c|c|c|c|}
\hline \multicolumn{5}{|l|}{ A. PAQLQ score } \\
\hline & Overall & & Symptoms & \\
\hline & $\mathrm{MD}(95 \% \mathrm{CI})$ & SMD $(95 \%$ CI $)$ & $\mathrm{MD}(95 \% \mathrm{CI})$ & SMD $(95 \%$ CI $)$ \\
\hline $\begin{array}{l}0 \text { versus 3-4 } \\
\text { triggers }\end{array}$ & $\mathbf{0 . 6 6}(0.57$ to 0.76$)$ & 0.74 (0.63 to 0.85$)$ & $\mathbf{0 . 8 6}$ (0.75 to 0.97$)$ & 0.82 (0.71 to 0.93 ) \\
\hline $\begin{array}{l}0 \text { versus } \geq 5 \\
\text { triggers }\end{array}$ & $\mathbf{1 . 0 2}$ (0.93 to 1.10$)$ & 1.10 (1.00 to 1.21$)$ & 1.26 (1.16 to 1.36$)$ & 1.20 (1.09 to 1.31$)$ \\
\hline $\begin{array}{l}1-2 \text { versus } \geq 5 \\
\text { triggers }\end{array}$ & $\mathbf{0 . 6 3}$ (0.54 to 0.73$)$ & 0.65 (0.55 to 0.75$)$ & $\mathbf{0 . 7 1}$ (0.60 to 0.83 ) & 0.62 (0.52 to 0.72$)$ \\
\hline $\begin{array}{l}0 \text { versus 1-2 } \\
\text { triggers }\end{array}$ & 0.38 (0.29 to 0.47$)$ & 0.47 (0.36 to 0.59$)$ & 0.55 (0.44 to 0.65$)$ & 0.59 (0.47 to 0.70$)$ \\
\hline $\begin{array}{l}1-2 \text { versus 3-4 } \\
\text { triggers }\end{array}$ & 0.28 (0.18 to 0.38$)$ & 0.29 (0.18 to 0.40$)$ & 0.31 (0.19 to 0.44$)$ & 0.27 (0.16 to 0.38$)$ \\
\hline \multirow[t]{3}{*}{$\begin{array}{l}3-4 \text { versus } \geq 5 \\
\text { triggers }\end{array}$} & 0.35 (0.25 to 0.45$)$ & 0.35 (0.25 to 0.44$)$ & 0.40 (0.28 to 0.52$)$ & 0.33 (0.23 to 0.42$)$ \\
\hline & Activities & & Emotions & \\
\hline & MD (95\% CI) & SMD $(95 \%$ CI $)$ & $\mathrm{MD}(95 \% \mathrm{CI})$ & $\operatorname{SMD}(95 \% \mathrm{CI})$ \\
\hline $\begin{array}{l}0 \text { versus 3-4 } \\
\text { triggers }\end{array}$ & $\mathbf{0 . 7 7}(0.65$ to 0.88$)$ & 0.70 (0.59 to 0.82 ) & 0.35 (0.26 to 0.44$)$ & 0.43 (0.32 to 0.54$)$ \\
\hline $\begin{array}{l}0 \text { versus } \geq 5 \\
\text { triggers }\end{array}$ & $\mathbf{1 . 2 4}$ (1.13 to 1.35$)$ & 1.07 (0.96 to 1.18 ) & $\mathbf{0 . 5 7}$ (0.48 to 0.65$)$ & 0.62 (0.52 to 0.73$)$ \\
\hline $\begin{array}{l}1-2 \text { versus } \geq 5 \\
\text { triggers }\end{array}$ & $\mathbf{0 . 8 9}$ (0.78 to 1.01$)$ & 0.76 (0.65 to 0.86$)$ & 0.37 (0.28 to 0.46$)$ & $0.40(0.30$ to 0.50$)$ \\
\hline $\begin{array}{l}0 \text { versus } 1-2 \\
\text { triggers }\end{array}$ & 0.35 (0.23 to 0.47$)$ & 0.34 (0.23 to 0.45$)$ & 0.20 (0.11 to 0.28$)$ & $0.26(0.15$ to 0.38$)$ \\
\hline $\begin{array}{l}1-2 \text { versus } 3-4 \\
\text { triggers }\end{array}$ & 0.42 (0.30 to 0.54$)$ & 0.37 (0.26 to 0.48$)$ & 0.15 (0.06 to 0.24$)$ & 0.18 (0.07 to 0.29$)$ \\
\hline $\begin{array}{l}3-4 \text { versus } \geq 5 \\
\text { triggers }\end{array}$ & 0.48 (0.36 to 0.59$)$ & 0.39 (0.30 to 0.49$)$ & 0.22 (0.13 to 0.31$)$ & 0.23 (0.13 to 0.32 ) \\
\hline \multicolumn{5}{|l|}{ B. RAND score } \\
\hline & MD $(95 \%$ CI) & $\operatorname{SMD}(95 \% \mathrm{CI})$ & & \\
\hline $\begin{array}{l}0 \text { versus 3-4 } \\
\text { triggers }\end{array}$ & 2.12 (1.60 to 2.63 ) & $0.45(0.34$ to 0.56$)$ & & \\
\hline $\begin{array}{l}0 \text { versus } \geq 5 \\
\text { triggers }\end{array}$ & 3.16 (2.67 to 3.65$)$ & 0.68 (0.57 to 0.78$)$ & & \\
\hline $\begin{array}{l}1-2 \text { versus } \geq 5 \\
\text { triggers }\end{array}$ & 2.69 (2.26 to 3.13 ) & 0.62 (0.27 to 0.72$)$ & & \\
\hline $\begin{array}{l}0 \text { versus } 1-2 \\
\text { triggers }\end{array}$ & $0.47(-0.06$ to 0.99$)$ & $0.10(-0.01$ to 0.21$)$ & & \\
\hline $\begin{array}{l}1-2 \text { versus } 3-4 \\
\text { triggers }\end{array}$ & 1.65 (1.18 to 2.12 ) & 0.38 (0.27 to 0.49$)$ & & \\
\hline $\begin{array}{l}3-4 \text { versus } \geq 5 \\
\text { triggers }\end{array}$ & 1.04 (0.61 to 1.48$)$ & 0.23 (0.14 to 0.33$)$ & & \\
\hline
\end{tabular}


Supplemental Table 4. Linear regression analysis for asthma-specific quality of life per domain

\begin{tabular}{|c|c|c|c|c|c|c|}
\hline & \multicolumn{3}{|c|}{ Symptoms } & \multicolumn{3}{|c|}{ Activities } \\
\hline & $a \beta^{+}$ & $95 \% \mathrm{CI}$ & $P$-value & $a \beta^{+}$ & $95 \% \mathrm{CI}$ & $P$-value \\
\hline \multicolumn{7}{|l|}{ Allergic triggers } \\
\hline Dust & -0.13 & -0.33 to 0.06 & 0.182 & 0.00 & -0.20 to 0.20 & 0.991 \\
\hline Pollen & 0.15 & -0.06 to 0.36 & 0.173 & 0.20 & -0.02 to 0.42 & 0.069 \\
\hline Pets & 0.01 & -0.20 to 0.21 & 0.950 & -0.23 & -0.44 to -0.02 & 0.030 \\
\hline \multicolumn{7}{|c|}{ Non-allergic triggers } \\
\hline Physical exercise & -0.21 & -0.40 to -0.02 & 0.027 & -0.21 & -0.40 to -0.01 & 0.038 \\
\hline The weather & -0.17 & -0.37 to 0.02 & 0.078 & -0.25 & -0.45 to -0.05 & 0.015 \\
\hline Cold or the flu & -0.09 & -0.27 to 0.10 & 0.349 & 0.01 & -0.18 to 0.21 & 0.887 \\
\hline (Cigarette) smoke & -0.34 & -0.58 to -0.11 & 0.004 & -0.30 & -0.54 to -0.06 & 0.016 \\
\hline Emotions & -0.21 & -0.45 to 0.04 & 0.102 & -0.23 & -0.49 to 0.02 & 0.076 \\
\hline Food or drinks & -0.13 & -0.39 to 0.13 & 0.335 & -0.22 & -0.50 to 0.05 & 0.111 \\
\hline Detergent & 0.04 & -0.27 to 0.34 & 0.805 & -0.14 & -0.45 to 0.18 & 0.398 \\
\hline \multirow[t]{3}{*}{ Wool clothing } & 0.29 & -0.10 to 0.68 & 0.150 & 0.25 & -0.15 to 0.66 & 0.222 \\
\hline & \multicolumn{3}{|c|}{ Emotions } & & & \\
\hline & $a \beta^{+}$ & $95 \% \mathrm{CI}$ & $P$-value & & & \\
\hline \multicolumn{7}{|l|}{ Allergic triggers } \\
\hline Dust & -0.08 & -0.25 to 0.09 & 0.335 & & & \\
\hline Pollen & 0.12 & -0.06 to 0.30 & 0.200 & & & \\
\hline Pets & 0.06 & -0.11 to 0.24 & 0.485 & & & \\
\hline \multicolumn{7}{|c|}{ Non-allergic triggers } \\
\hline Physical exercise & -0.06 & -0.23 to 0.10 & 0.451 & & & \\
\hline The weather & -0.12 & -0.28 to 0.05 & 0.171 & & & \\
\hline Cold or the flu & 0.09 & -0.07 to 0.25 & 0.279 & & & \\
\hline (Cigarette) smoke & -0.17 & -0.37 to 0.04 & 0.106 & & & \\
\hline Emotions & -0.41 & -0.63 to -0.20 & 0.000 & & & \\
\hline Food or drinks & -0.11 & -0.34 to 0.12 & 0.344 & & & \\
\hline Detergent & 0.19 & -0.07 to 0.46 & 0.147 & & & \\
\hline Wool clothing & 0.12 & -0.22 to 0.46 & 0.483 & & & \\
\hline
\end{tabular}


Impact of asthma triggers on quality of life 
<smiles>c1ccccc1</smiles> 


\section{Chapter 9}

General discussion and summary 


\section{Main findings}

The aim of this thesis was to improve the diagnostics and quality of life in patients with atopic diseases, particularly in patients with peanut allergy and asthma.

\section{Part I. The diagnostics of atopic diseases}

- The previously published cutoff level of specific IgE (SIgE) to peanut component Ara h 2 is validated in secondary and tertiary care (chapter 2).

- A diagnostic flowchart based on sIgE to Ara h 2 is accurate, safe and reduces costs in children with suspected peanut allergy in secondary and tertiary care (chapter 2).

- Sensitization to Ara h 2 and Ara h 6 predicts peanut allergy correctly in one third of adults with suspected peanut allergy in tertiary care (chapter $\mathbf{3}$ ).

- Despite confirmed peanut-allergy diagnosis by a double-blind placebocontrolled food challenge (DBPCFC) and careful instruction and guidance, almost half of children have peanut-allergic reactions during the 3 year follow-up after the DBPCFC. One third of children fail to introduce peanut after a negative DBPCFC result (chapter 4).

- Risk factors for atopic diseases are distinct from risk factors for recurrent respiratory tract infections, suggesting that the changing prevalence of both entities is not related to shared risk factors (chapter $\mathbf{5}$ ).

\section{Part II. The impact of atopic diseases on patients' welfare}

- A clear food allergy diagnosis is associated with reduced parental anxiety, improved health-related quality of life (HRQL) and reduced parental burden of food allergy (chapter 2, 4 and 6).

- During 3-year follow-up after a negative DBPCFC for peanut, HRQL improved in children who consumed peanut and not in children who failed to consume peanut (chapter 4).

- In secondary care, uncontrolled asthma is highly prevalent in children referred for asthma and in children referred for other atopic diseases than asthma (chapter 7).

- A higher number of perceived asthma triggers is associated with reduced HRQL in children with asthma (chapter 8). 


\section{The diagnostics of atopic diseases}

In the first part of this thesis we focused on improving diagnostics in patients with atopic diseases, particularly in patients with suspected peanut allergy. We validated cutoff levels of specific IgE (sIgE) to Ara h 2 and implemented a diagnostic flowchart based on these cutoff levels in children in secondary and tertiary care. Furthermore, we evaluated the diagnostic value of peanut components and validated the Ara h 2 cutoff level in adults in tertiary care. Thirdly, we investigated risk factors for atopic diseases and for recurrent respiratory tract infections.

\section{Peanut allergy diagnostics}

Peanut allergy is one of the most common food allergies in childhood, affecting almost $25 \%$ of food allergic children. ${ }^{1}$ Peanut allergy represents one of the most common elicitors of anaphylaxis ${ }^{2}$, and is outgrown in less than $20 \%$ of children. ${ }^{3}$ The significant personal and societal burden of peanut allergies could be reduced to some extent by preventing overdiagnosis. ${ }^{4-6}$ Previous data showed that the prevalence of self-reported peanut allergy is considerably higher than the prevalence of challenge-proven peanut allergy, with an estimated point prevalence of respectively $1.67 \%$ and $0.22 \%{ }^{7}$

In current guidelines and in a recent review summarizing diagnostic modalities for peanut allergy, it is stated that an oral food challenge is still the reference standard for diagnosing peanut allergy ${ }^{8-10}$ Furthermore, the implementation of componentresolved diagnostics (CRD), including sIgE to peanut component Ara $\mathrm{h} 2$, is thus far not recommended as validation in daily practice has not yet been studied. In addition, most studies investigating the diagnostic performance of CRD were performed in tertiary care and the question was whether results of these studies were generalizable to other lines of care.

\section{Accurate and safe peanut allergy diagnostics in children}

With the results of this thesis, we demonstrated that cutoff levels of Ara $\mathrm{h} 2$ with $100 \%$ positive and negative predictive value, as observed in previous research in our tertiary care center, are ready for implementation in daily practice in secondary and tertiary care (chapter 2). We showed that the diagnostic flowchart accurately confirmed (89\% to $97 \%)$ and excluded (100\%) peanut allergy in children aged 3.5 to 18 years with suspected peanut allergy. The diagnostic flowchart, based on Ara h 2 cutoff levels as observed in previous research conducted mainly in our tertiary care center, was accurate in both secondary and tertiary care. ${ }^{11}$ These findings suggest that Ara $\mathrm{h} 2$ cutoff levels as observed in tertiary care can also be used to accurately predict peanut allergy in secondary care. ${ }^{12}$ The oral food challenge results in tertiary care were compared to the results in secondary care in a large validation 
study in children in New Zealand and no differences were observed regarding patient demographics, severity and the rate of inconclusive challenges.

In line with these results, we observed no differences between children in secondary care and tertiary care in our cohort regarding age, gender, reason for suspected peanut allergy, severity of the previous reaction and severity of symptoms during the oral food challenge. These data support the generalizability of (peanut) allergy diagnostics from tertiary to secondary care.

In our implementation study as described in chapter 2, all children with sIgE to Ara h $2 \leq 0.1 \mathrm{kU}_{\mathrm{A}} / \mathrm{L}$ were peanut-tolerant (i.e. $100 \%$ negative predictive value). During peanut introduction, none of these children experienced any allergic symptoms. These findings endorse the safety of the diagnostic flowchart and stimulate introduction of peanut at home in children with an Ara $\mathrm{h} 2$ level $\leq 0.1 \mathrm{kU}_{\mathrm{A}} / \mathrm{L}$. However, only in one third of children with an Ara $h 2$ level $\leq 0.1 \mathrm{kU}_{\mathrm{A}} / \mathrm{L}$ the physician and/or parents initiated home introduction. The remaining two third of children introduced peanut in the hospital, mainly because the child or parent was anxious or because the physician regarded the clinical history as suggestive for peanut allergy. It must be noted that a suggestive clinical history was not associated with peanut allergy in children with an Ara $\mathrm{h} 2$ level $\leq 0.1 \mathrm{kU}_{\mathrm{A}} / \mathrm{L}$. Five children reported a severe allergic reaction in clinical history, but were all tested negative (i.e. peanut-tolerant). We strongly hope that our results provide confidence to physicians to stimulate home introduction in children with an Ara h 2 level $\leq 0.1 \mathrm{kU}_{\mathrm{A}} / \mathrm{L}$. In previous research, rare cases of peanut allergy have been

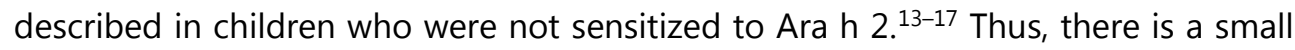
probability of peanut allergy in children with no detectable sensitization to Ara $\mathrm{h} 2$, which may be explained by sensitization to other peanut components (e.g. Ara $\mathrm{h} 6$ or Ara $\mathrm{h}$ 7) or sensitization to unidentified allergens. In this regard, careful guided introduction at home using a schedule with increasing amounts of peanut is recommended in children with negative Ara $\mathrm{h} 2$ levels. As anxiety was the main reason to decline home introduction, clinicians are encouraged to identify patients with excessive anxiety and subsequently, assess the impact via anxiety and HRQL questionnaires. In children or parents with excessive anxiety who decline home introduction of peanut, a short introduction schedule of peanut at the outpatient clinic may be a good alternative. In case of persisting anxiety, psychological support should be offered.

Children with an Ara h 2 level $\geq 5.0 \mathrm{kU}_{\mathrm{A}} / \mathrm{L}$ were highly likely to be peanut-allergic: the positive predictive value (PPV) ranged between $89 \%$ and $97 \%$. Previous diagnostic studies among Dutch children report a 100\% PPV for Ara h $2 \geq 5.0 \mathrm{kU}_{\mathrm{A}} / \mathrm{L}$ in tertiary care and $\geq 5.61 \mathrm{kU} / \mathrm{L}$ in secondary care. ${ }^{11,18}$ Although the PPV was not $100 \%$, the implementation of the diagnostic flowchart did not lead to overdiagnosis of peanut allergy as all children were offered a 1-day open oral food challenge. 
According to the European food allergy guideline, the design of the OFC, either open or double-blind, depends on the indication and purpose for which the food challenge is being performed. ${ }^{9}$ As children with an Ara $\mathrm{h} 2$ level $\geq 5.0 \mathrm{kU} / \mathrm{L}$ have a very high probability of being peanut-allergic, the purpose of the OFC is to gain insight into the severity of peanut allergy and the threshold of the peanut-allergic reaction. Open OFCs were feasible in our cohort, as inconclusive challenges were infrequent (8\%) and these inconclusive challenges were due to incomplete ingestion of the challenge material. Incomplete ingestion is equally likely, or even more likely to occur, when challenged double-blind.

It must be noted that the measures of diagnostic accuracy, including positive and negative predictive values, are dependent on the population (e.g. disease prevalence, age, setting and country).

\section{sIgE to Ara $h \mathbf{2}$ predicts peanut allergy correctly in one third of adults}

In adults with suspected peanut allergy, sensitization to peanut component Ara h 2 predicts peanut allergy correctly in one third of patients (chapter 3). All patients with levels of Ara $h 2 \geq 1.75 \mathrm{kU}_{\mathrm{A}} / \mathrm{L}$ were peanut-allergic. These results validate the cutoff level of Ara h 2 assessed in a previous diagnostic study performed in our center. ${ }^{19}$ In addition, we were the first to determine an Ara $\mathrm{h} 6$ cutoff level with a $100 \%$ PPV to confirm peanut allergy in adults (i.e. $\geq 1.80 \mathrm{kU}_{\mathrm{A}} / \mathrm{L}$ ). sIgE to Ara $\mathrm{h} 2$ and Ara $\mathrm{h} 6$ were the best individual predictors of peanut allergy in adults in our cohort. As the concentrations of sIgE to Ara h 2 and Ara h 6 were highly correlated, and Ara h 2 was validated, we developed a diagnostic flowchart for adults with suspected peanut allergy based on sIgE to Ara h 2 (Figure 1). Again, it must be noted that the measures of diagnostic accuracy as depicted in the diagnostic flowchart are dependent on the population and further studies are needed to evaluate whether the flowchart is generalizable to other countries or other settings.

In comparison to children, the same percentage of peanut-allergic patients can be identified using a cutoff level of Ara h 2 with 100\% positive predictive value (PPV). However, the cutoff level to confirm peanut allergy is lower in adults (i.e. Ara $\mathrm{h} 2 \geq$ $1.75 \mathrm{kU}_{\mathrm{A}} / \mathrm{L}$ ) compared to children (i.e. Ara h $2 \geq 5.0 \mathrm{kU}_{\mathrm{A}} / \mathrm{L}$ ). ${ }^{11}$ Furthermore, although sensitization to peanut components can be used to exclude peanut allergy in children, it cannot be used to exclude peanut allergy in adults. These differences between peanut allergy diagnostics in children and adults may be partly explained by the higher proportion of peanut allergy with subjective symptoms only in adults, due to birch pollen-related peanut allergy. ${ }^{19-21}$ Peanut allergy based on subjective symptoms only was observed in $12.5 \%$ of children (chapter 2 ) and $27 \%$ of adults (chapter 3). Previous research showed that sensitization to peanut storage proteins (including Ara h 1, 2 and 3) decreases with age, while sensitization to Ara h 8 and birch pollen increases. ${ }^{22,23}$ In our study, adults with peanut allergy based on 
subjective symptoms only had lower titers of sIgE to Ara h 2 and Ara h 6, resulting in a lower diagnostic value of these components. Furthermore, we did not detect sensitization to Ara h 1, 2, 3 and 6 in 16 (17\%) peanut-allergic adults included in our study. Thus, in part of the adult population, peanut allergy might be caused by sensitization to thus far unidentified peanut components. Additional insight into the reason for the difference in diagnostic performance of peanut components between children and adults may arise from evaluation of the sIgE levels over time from childhood to adulthood.
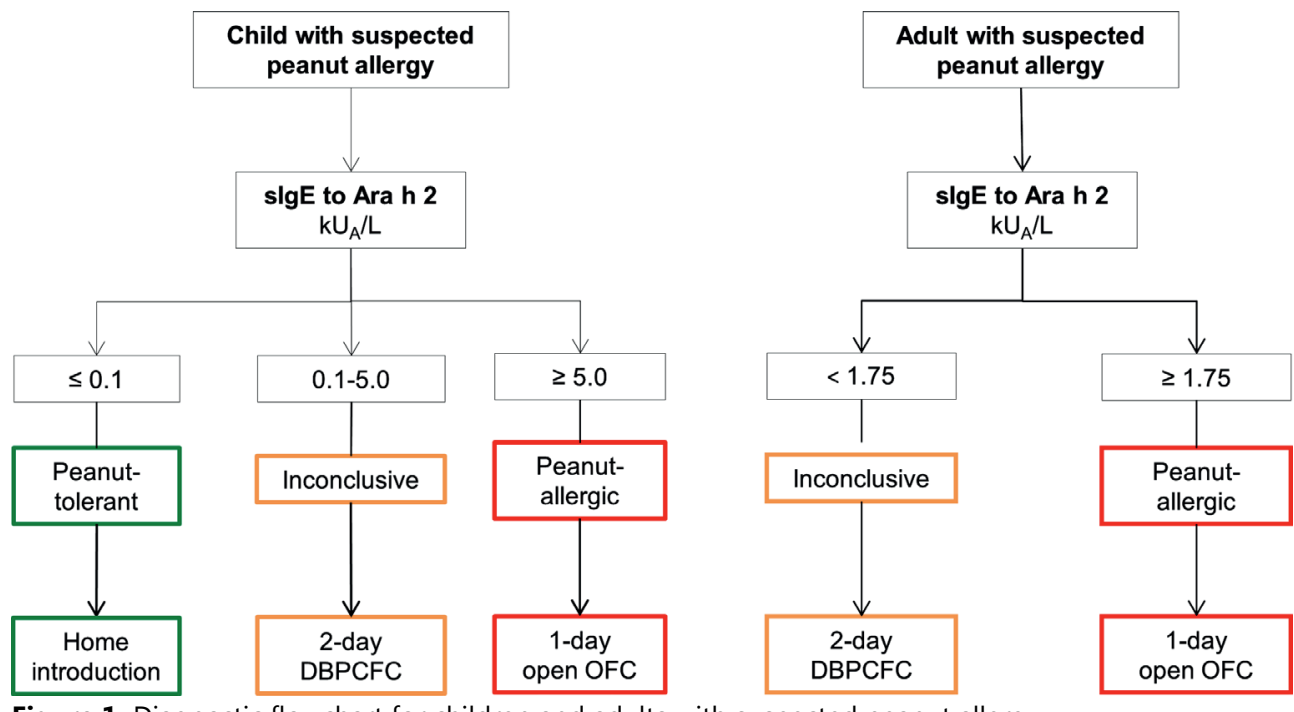

Figure 1. Diagnostic flowchart for children and adults with suspected peanut allergy

Abbreviations: DBPCFC, double-blind placebo-controlled food challenge; OFC, oral food challenge; slgE, specific IgE.

\section{The costs of food allergy}

Food allergy is also a financial burden to patients and families, the health care system and society. Direct medical costs borne by the health care system include the costs for the diagnosis and treatment of food allergy. The total annual direct medical costs of food allergy were estimated to be mean $\$ 2,081$ per child in a recent meta-analysis. ${ }^{24}$ The direct costs of food allergy are comparable to the costs of asthma ${ }^{25}$ and atopic dermatitis ${ }^{26}$, but higher than the costs of allergic rhinitis ( $\$ 233$ to $\$ 657$ per patient). ${ }^{27,28}$ The indirect costs of food allergy include costs due to productivity losses and have been estimated at mean $\$ 1,038$ per patient. ${ }^{24}$ These costs are comparable to the costs of atopic dermatitis ${ }^{26}$, but are higher than the costs of asthma ( $\$ 207$ per patient) $)^{29}$ and allergic rhinitis ( $\$ 833$ per patient). ${ }^{27}$ Finally, the individual out-of-pocket costs of food allergy were estimated to be mean $\$ 1,874$ per patient. ${ }^{24}$ The out-of-pocket costs of food allergy are again comparable to atopic dermatitis ${ }^{30}$, but higher than the costs of asthma ( $\$ 154$ per patient) ${ }^{31}$ and allergic rhinitis ( $\$ 50$ per patient). ${ }^{32}$ However, comparing the costs between disease 
entities should be done with care. The estimated costs are affected by a number of factors, including differences in healthcare and insurance system, country and year of study.

\section{A diagnostic flowchart reduces costs of peanut allergy diagnostics}

We aimed to reduce the costs of food allergy by improving peanut allergy diagnostics and reducing the number of oral food challenge days. According to the current Dutch national guideline, children with sensitization to peanut (i.e. SIgE to peanut extract $\geq 0.35 \mathrm{kU}_{\mathrm{A}} / \mathrm{L}$ or skin prick test $\geq 3 \mathrm{~mm}$ ) are supposed to undergo a double-blind placebo-controlled food challenge (DBPCFC). According to our diagnostic flowchart, no oral food challenges are needed for children with an Ara $h$ 2 level $\leq 0.1 \mathrm{kU}_{\mathrm{A}} / \mathrm{L}$ as these children have a very high probability of being peanuttolerant. Furthermore, children with an Ara $\mathrm{h} 2$ level $\geq 5.0 \mathrm{kU}_{\mathrm{A}} / \mathrm{L}$ have a very high probability of being peanut-allergic and thus, the oral food challenge can be performed in one day as previously explained. Compared to the current national guideline, the diagnostic flowchart could lead to a reduction in oral food challenge days up to $60 \%$.

In chapter 2, we demonstrated that the annual direct and indirect costs of peanut allergy diagnostics in children according to the diagnostic flowchart were $€ 320$ to $€ 636$ lower per child compared to the costs when following the current national guideline. With an estimated 2400 referred children per year in the Netherlands, nationwide implementation of the diagnostic flowchart could lead to a cost reduction of $€ 767,101$ to $€ 1,526,688$ per year. ${ }^{7,33,34}$ A previous cost-effectiveness analysis in Norway supports our findings and showed that costs of a diagnostic flowchart based on multiple peanut components, including Ara h 2, were lower compared to current clinical practice based on SIgE to peanut extract and oral food challenges. ${ }^{35}$ Moreover, the implementation of a diagnostic flowchart based on SIgE to Ara $\mathrm{h} 2$ in adults could even further reduce the costs of peanut allergy diagnostics. According to our diagnostic flowchart, approximately $30 \%$ of adults can be challenged in one day instead of two days. With an estimated 1800 referrals of adults with suspected peanut allergy per year in the Netherlands, nationwide implementation of the diagnostic flowchart in adults could prevent at least 540 food challenge days for peanut allergy diagnostics. 733,34

\section{Frequent accidental reactions occur after positive peanut allergy diagnostics}

In chapter 4, we performed a three-year follow-up of 83 children who had a confirmed or excluded peanut allergy after a DBPCFC. ${ }^{11}$ The longitudinal study design enables insight into potential changes over time and long-term impact of diagnostic interventions. ${ }^{36}$ We noted that almost half of peanut-allergic children experienced one or more allergic reaction(s) to peanut during the three years after their peanut DBPCFC, although all these children were advised and instructed to 
avoid peanut. Other studies have shown that accidental allergic reactions to peanuts in patients on an avoidance diet are common. ${ }^{37}$ Accidental allergic reactions may be caused by the consumption of products with hidden sources of peanut, by incomplete or incorrect labelling, by misreading of the labels or because of risky behavior. ${ }^{38,39}$ Accidental allergic reactions may be prevented by improved food allergen labelling and, when applicable, by addressing risk-taking behavior. Furthermore, children with severe allergic reactions did not use their epinephrine auto-injector despite instructions on how and when to use the auto-injector. Previous research showed underutilization of epinephrine auto-injectors by foodallergic patients. ${ }^{40,41}$ One of the most common reasons for not using the autoinjector is failure to recognize symptoms of anaphylaxis. ${ }^{42}$ Delayed administration of epinephrine in patients with anaphylaxis is associated with an increased risk of hospitalization and fatality. ${ }^{43}$ The adequate use of epinephrine auto-injectors in patients with anaphylaxis may be improved by repeated practical demonstrations and training and education of both patients and health care professionals..$^{44,45}$

\section{Frequent introduction failure after negative peanut allergy diagnostics}

One third of peanut-tolerant children failed to introduce peanut into the diet during three years after a negative peanut DBPCFC, although all families were counseled after the DBPCFC regarding the importance and practical details of introduction (chapter 4). In previous studies, continued food avoidance despite negative food challenge results were reported in $25 \%$ to $38 \%$ of children. ${ }^{46-49}$ Children failed to consume peanut mainly because of aversion. Aversion may be partly explained by the fact that these children are not used to the taste and texture of peanuts or because the taste of peanuts has previously been paired with (severe) allergic symptoms. However, consumption of peanut may be beneficial for patients and parents as we observed an improved parent-proxy reported healthrelated quality of life in peanut-tolerant children after successful peanut introduction. Health-related quality of life of peanut-tolerant children who failed to introduce peanut remained unchanged. Further research should investigate how to improve the rate of peanut introduction. As an example, behavioral interventions designed for children with pediatric feeding disorders might be indicated in peanut-tolerant children with aversion to peanut. ${ }^{50}$

\section{Future aspects of peanut allergy diagnostics}

A diagnostic flowchart based on peanut component Ara $\mathrm{h} 2$ is an accurate and safe diagnostic tool in secondary and tertiary care in the Netherlands. We recommend implementation of the diagnostic flowchart in the current national guideline, both for children and adults with a suspected peanut allergy. As previously mentioned, the cutoff levels of peanut components are not fixed, but dependent on the population. International validation studies are needed to implement the (if applicable: adapted) diagnostic flowchart worldwide. 
This thesis demonstrates that more than half of children and one third of adults can be classified correctly using sensitization to peanut component Ara h 2. To further increase the proportion of correctly classified patients, future studies should evaluate diagnostic tools for children and adults with 'inconclusive' levels of Ara $\mathrm{h} 2$ (Ara $\mathrm{h} 2$ levels between 0.1 and $5.0 \mathrm{kU}_{\mathrm{A}} / \mathrm{L}$ and $<1.75 \mathrm{kU}_{\mathrm{A}} / \mathrm{L}$, respectively) and for peanut-allergic adults not showing sensitization to peanut components. A previous study demonstrated that half of children with Ara $\mathrm{h} 2$ levels between 0.1 and 5.0 $\mathrm{kU}_{\mathrm{A}} / \mathrm{L}$ could be classified correctly when using the Basophil Activation Test (BAT) as a second step in the diagnostic process. ${ }^{11}$ As the BAT is not yet used in daily practice due to practical, technical and analytical limitations $s^{51}$, future studies should focus on standardization, validation and automation of this procedure. Furthermore, the diagnostic value of the Mast cell Activation Test (MAT) has been evaluated..$^{52}$ The MAT might be superior to other existing diagnostic test modalities, including sIgE to Ara h 2 and the BAT, but further research is needed to validate these findings and facilitate implementation in daily practices. In addition, patterns of IgE binding to specific regions of an allergen (i.e. allergenic epitopes) in microarray immunoassays may become a future diagnostic tool. Peanut-allergic patients showed more IgE epitope binding and broader epitope diversity compared with peanut-tolerant patients in previous studies..$^{53,54}$ In addition, the analysis of patient-derived monoclonal antibodies may provide diagnostic insight after further development. This approach could provide information on epitope affinity and specificity. ${ }^{55}$ Finally, understanding potentially allergy-promoting lipids may improve serum-based allergy diagnostics. Lipids may enhance the allergenicity of proteins in several ways, e.g. by enhancing an IgE dominant response or by influencing the ability of allergens to penetrate through the oral, respiratory or gastrointestinal mucosa cells. 56,57

It must be noted that current diagnostic IgE test modalities fail to predict the threshold of an allergic reaction and the severity of peanut allergy of individual patients. The mechanisms driving the severity of food allergic reactions remain poorly understood. ${ }^{58,59}$ Future research, including immunologic, genetic and epigenetic analyses, may enhance our understanding of the reaction threshold and reaction severity and identify biomarkers for severe allergic reactions. As an example, recent research observed a correlation between an expansion of peanutspecific effector T-cells and high clinical reactivity (i.e. allergic reaction at a dose less than or equal to $300 \mathrm{mg}$ peanut protein) in peanut-allergic patients. ${ }^{60}$ Another recent study identified several genes and DNA methylation sites associated with reaction severity in peanut-allergic children. ${ }^{61}$

When considering different diagnostic strategies for peanut allergy, it is vital to take into account the wishes of parents and/or patients and make shared decisions. Patients may still prefer oral food challenges to laboratory testing, as they may wish 
to consume peanut in a safe environment and experience whether, when and what symptoms occur after peanut ingestion. In patients with high and conclusive Ara $\mathrm{h}$ 2 levels (i.e. Ara $\mathrm{h} 2 \geq 5.0 \mathrm{kU} / \mathrm{L}$ in children and $\geq 1.74 \mathrm{kU}_{\mathrm{A}} / \mathrm{L}$ in adults), the oral food challenge protocol may be adapted to reduce the burden and costs of food challenges.

Moreover, the future of peanut allergy diagnostics is likely changed by emerging prospects for prevention and treatment of peanut allergy.

\section{Prevention of peanut allergy}

In 2015, the Learning Early About Peanut Allergy trial clearly showed that peanut introduction early in life significantly decreased the risk of peanut allergy among infants with high-risk atopic disease(s) (i.e. severe atopic dermatitis, egg allergy or both). ${ }^{64}$ The trial received wide publicity and new peanut allergy prevention guidelines were developed. ${ }^{65-67}$ The paradigm shift in the timing of food allergen introduction has implications for future peanut allergy diagnostics. Early introduction of peanut may likely lower the prevalence of peanut allergy, but may also increase the frequency of diagnostic testing at a younger age. Previous data showed that sIgE to storage proteins, including Ara $\mathrm{h} 2$, is present in children below the age of 4 years. ${ }^{17,68}$ However, as our diagnostic flowchart is based on sIgE to Ara h 2 cutoff levels in children aged 3.5 to 18 years, further research is necessary to confirm or adapt Ara h 2 cutoff levels in younger children.

\section{Treatment of peanut allergy}

Our long-term follow-up of peanut-allergic children showed that living with a peanut allergy remains a challenge during childhood. Previous studies have observed the same in adulthood. ${ }^{38}$ The current management of peanut allergy includes (instructions on) an elimination diet, instructions on and prescription of emergency medication and advice for timely administration of emergency medication when an allergic reaction occurs. ${ }^{9}$ In recent years, oral or epicutaneous immunotherapy for the treatment of peanut allergy has been the subject of much research. ${ }^{69,70}$ In January 2020, the first oral immunotherapy product has been approved by the United States Food and Drug Administration. ${ }^{71}$ Immunotherapy relies on the delivery of gradually increased doses of the allergenic food to increase the threshold of an allergic reaction during treatment (i.e. desensitization) and after treatment (i.e. sustained unresponsiveness). ${ }^{72-74}$ Recent studies found that the primary motivation for undergoing oral immunotherapy was to reduce the risk of fatal allergic reactions. ${ }^{75}$ Parents who perceived their child to be at high risk of fatal food allergic reactions were more likely to enroll in peanut immunotherapy compared with routine clinical care (i.e. peanut avoidance). ${ }^{76}$ However, a recent systematic review and meta-analysis showed that current peanut oral immunotherapy regimens increased the risk of allergic reactions, including 
anaphylaxis, compared with peanut avoidance. ${ }^{77}$ Low-dose oral or epicutaneous immunotherapy may better align with families' goals. Overall, carefully outweighing the pros versus the cons of immunotherapy for food allergy is paramount in deciding the appropriate strategy per patient. ${ }^{37}$ Further research is warranted to evaluate long-term results and patients' perspectives.

\section{Risk factors for atopic diseases differ from risk factors for recurrent infections}

In this thesis, the association between risk factors and atopic diseases and between risk factors and recurrent respiratory tract infections (RRTI) was investigated in a large cohort of children aged 1 to 18 years (chapter 5). The results demonstrate that the demographic and environmental risk factors associated with atopic diseases largely differ from the risk factors associated with RRTI. Interestingly, family history was a disease-specific risk factor for atopic diseases and RRTI.

A family history of atopic diseases and RRTI reflects genetic susceptibility, as well as shared environmental and behavioral factors. ${ }^{78}$ Clustering of diseases in families is typically due to multifactorial inheritance, determined by a number of genes in interaction with environmental and lifestyle factors, and not due to single genetic mutations. Strong evidence for the importance of genetic factors in the susceptibility to atopic diseases comes from studies among genetically identical twins, indicating a concordance rate of approximately $81 \%$ for food allergy, $95 \%$ for asthma, $91 \%$ for allergic rhinitis and $84 \%$ for atopic dermatitis. ${ }^{79}$ Furthermore, atopic diseases are to a large extent genetically related. ${ }^{80}$ However, our and previous studies indicate that some genetic risk factors are disease-specific. ${ }^{81}$ The genetic correlations between atopic diseases have been estimated $<1$, suggesting that there are unique genetic influences to each atopic disease. ${ }^{82,83}$ In addition, the association between family history and RRTI requires further investigator, as it has not been described before. Previous literature reported an association between family history and specific genetic diseases (e.g. primary immunodeficiencies, cystic fibrosis and primary ciliary dyskinesia). ${ }^{84-86}$ Given the rarity of these diseases, the association between a family history and RRTI as observed in our cohort is likely explained by a multifactorial, polygenic inheritance pattern.

A family history can be easily assessed and used for the prevention, detection and management of disease. ${ }^{87}$ Therefore, the family history of atopic diseases and recurrent respiratory tract infections should be assessed by physicians in clinical practice to identify high-risk children. 


\section{The impact of atopic diseases on patients' welfare}

The evaluation of the patient's perception of their health is an important aspect of patient-centered care. The patient's perception can be evaluated using patientreported outcome measures (PROMs). ${ }^{88}$ An example of frequently used PROMs are standardized and validated questionnaires on anxiety and health-related quality of life (HRQL) as administered in several studies described in this thesis.

Children with food allergy have poorer HRQL than children in the general population, due to multiple disease-specific impairments such as social and dietary restrictions, the need to carry an epinephrine auto-injector and the fear of accidental allergic reactions. ${ }^{89,90}$ Compared to other diseases, children with food allergy showed more impairment in HRQL than children with chronic liver disease or diabetes mellitus, but less impairment in HRQL than children with asthma. ${ }^{89,91}$ Especially children with uncontrolled asthma have impaired HRQL. ${ }^{92,93}$

In the second part of this thesis, we focused on improving the welfare of patients with atopic diseases, particularly children with peanut allergy and asthma. We studied anxiety and quality of life after peanut allergy diagnostics. Furthermore, we investigated the prevalence and predictors of uncontrolled asthma and the impact of asthma triggers on HRQL.

\section{Anxiety and quality of life improve after a clear diagnosis}

We summarized recent literature on the association between HRQL and food allergy diagnostics in chapter 6 and showed that oral food challenges are associated with improved HRQL and reduced parental burden, regardless of the food challenge result. The greatest improvement in HRQL was observed among children who demonstrated tolerance.

In chapter 2, we demonstrated a large reduction of parental anxiety after the telephonic consultation discussing an Ara $\mathrm{h} 2$ level $\leq 0.1 \mathrm{kU} / \mathrm{A} / \mathrm{L}$ (i.e. peanut-tolerant) and after oral food challenges, in the latter case even irrespective of the result. Although peanut allergy diagnosis is clear in children with an Ara $\mathrm{h} 2$ level $\geq 5.0$ $\mathrm{kU}_{\mathrm{A}} / \mathrm{L}$ (i.e. peanut-allergic), parental anxiety remained unchanged after the telephonic consultation. Uncertainty regarding the severity of peanut allergy and the threshold of an allergic reaction may be associated with anxiety, as reported in previous research. ${ }^{94}$ Uncertainty and anxiety may be reduced after an oral food challenge, as this procedure provides insight into these matters. Our and other studies have shown a reduction in food allergy-related anxiety after an oral food challenge, regardless of the result. ${ }^{94,95}$ These findings suggest that a clear diagnosis of food allergy may have beneficial psychosocial impact. However, the main reason to decline a food challenge or (home) introduction after the determination of SIgE 
to Ara h 2 was anxiety of the child and/or parents. Children or parents may experience anxiety or distress about the possibility of an allergic reaction during the procedure. ${ }^{94}$ Although some increased level of anxiety in patients with food allergy is an appropriate response, excessive anxiety should be recognized and treated. Mental health professionals may treat excessive anxiety through consultation or psychotherapy, or provide support during oral food challenges, as recommended in a recent position statement of the American Academy of Allergy, Asthma \& Immunology. ${ }^{96}$ Further work to study anxiety in food allergic children and the impact of psychological interventions needs to be done, as most studies focused on parents ${ }^{94,95,97,98}$ or adults. ${ }^{99}$ The benefit of psychological interventions has been reported in mothers of food allergic children. A brief cognitive behavioral therapy session was associated with significantly reduced anxiety after one year follow-up compared to no intervention, especially in mothers with high baseline anxiety levels. ${ }^{98}$

\section{Uncontrolled asthma is highly prevalent in children with asthma}

In chapter 7 it was demonstrated that uncontrolled asthma is highly prevalent in children referred for asthma (47\%), but also in children referred for other atopic diseases than asthma (26\%) to secondary care. We found a family history of asthma, recurrent upper or lower respiratory tract infections and a referral for asthma to be independently and significantly associated with uncontrolled asthma.

The current clinical guideline emphasizes that the main goal of asthma management is to attain and to keep good asthma symptom control. ${ }^{100}$ From previous studies we know that uncontrolled asthma is associated with healthrelated quality of life (HRQL); the better asthma control, the better HRQL. ${ }^{101,102}$ Patients with well-controlled asthma had similar HRQL as participants without asthma in a Swedish population-based cohort study. ${ }^{103}$ In addition to the patient burden, uncontrolled asthma poses a substantial and growing burden on healthcare systems. A recent study in the United States estimated that around $20 \%$ of direct costs of asthma can potentially be saved if all patients would achieve adequate asthma control. ${ }^{104}$ In order to reduce the patient and economic burden of uncontrolled asthma, an accurate review of the asthma control status is essential.

Our study indicates that assessment of disease control is not only important for children who are referred for asthma, but also for children who are referred for other atopic diseases than asthma. As patients and physicians tend to overestimate the level of asthma control ${ }^{105}$ and differ in their perception of asthma control ${ }^{106}$, standardized tools to monitor asthma control such as the Asthma Control Test are recommended at visits to the outpatient clinic. ${ }^{107,108}$ These tools should be used regularly, as asthma is characterized by marked fluctuations in symptoms over time. 


\section{Asthma triggers are associated with reduced health-related quality of life}

Asthma control is affected by various asthma triggers. For instance, strenuous physical exercise, bad weather, strong emotions or exposure to pollen or pets can trigger asthma symptoms. ${ }^{109}$ As described in chapter 8, more than three quarters of children with asthma report one or more perceived trigger(s) of asthma symptoms. The evaluated asthma triggers are depicted in Figure 2. Previous research identified an association between a higher number of asthma triggers and reduced asthma-specific HRQL in children with severe or difficult-to-treat asthma and in adults with asthma. ${ }^{110-113}$ We were able to confirm this association between asthma triggers and HRQL, both asthma-specific and generic, in children with asthma of varying severity in secondary and tertiary care. Children with a high number of asthma triggers may experience impaired HRQL due to an increased frequency of asthma exacerbations and health care visits. ${ }^{112-114}$ Furthermore, children may try to avoid asthma triggers which may have additional negative impact on HRQL. The asthma trigger 'emotions' was consistently associated with both asthma-specific and generic HRQL in our population. Previous work found that almost one third of asthmatic patients reported emotional triggers as the most frequent trigger of asthma symptoms. ${ }^{113}$ Examples of emotional triggers are stress, worry, feelings of loneliness or excitement and being angry. Interventions to reduce emotional distress may lower the impact of these triggers on HRQL.

The results of our study highlight the importance of asthma triggers in children with asthma. Physicians should evaluate the number and impact of asthma triggers on asthma symptoms in children with asthma. Further research is needed to evaluate the impact of identification, and subsequent avoidance and/or treatment, of asthma triggers on patient HRQL.

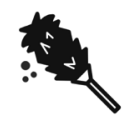

dust

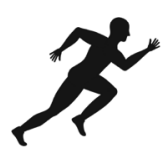

exercise

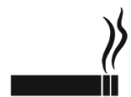

smoke

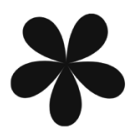

pollen

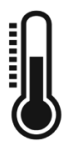

weather

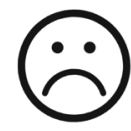

emotions

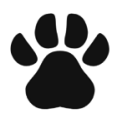

pets

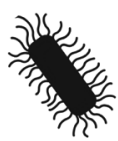

infections

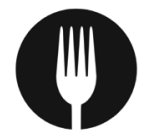

food

Figure 2. Asthma triggers 


\section{Future aspects}

'Health as the ability to adapt and to self-manage, in the face of social, physical and emotional challenges.'

Machteld Huber, $2011^{115}$

The traditional WHO definition of health formulated in 1948, as stated in the Introduction section of this thesis, has been debated ever since. ${ }^{116,117}$ Particularly the absoluteness of the words 'complete wellbeing' has been criticized. This formulation of health may contribute to the medicalization of society and is no longer considered adequate in an era with increasing numbers of people living with chronic diseases. A new concept of health was introduced by Machteld Huber and colleagues in $2011 .{ }^{115}$ The new, dynamic concept of health, as cited above, focuses on a person's resilience and ability to cope with new situations. The support for this new health concept was evaluated among various stakeholders, including patients and physicians. ${ }^{118}$ Physicians considered bodily functions and quality of life as the most important dimensions of health, where patients also considered societal, mental and spiritual dimensions as important dimensions.

The changing definition of the concept of health and the discrepancy between the perception of health by patients and by physicians requires attention. Future health-related quality of life (HRQL) research in patients with atopic diseases should attempt to validate questionnaires that measure various dimensions and aspects of health, including the burden of disease, coping, resilience, patients' satisfaction and disease-specific anxiety. In addition, psychosocial interventions to reduce anxiety and improve coping and resilience should be evaluated, as these actions may improve quality of life in patients with atopic diseases and their families.

Currently, HRQL is mainly assessed in research settings, while implementation in daily clinical practice is important to improve counseling and enable identification of high-risk children who could benefit from psychosocial interventions. Healthrelated quality of life should be assessed in both children and parents, as they differ in their view concerning HRQL. A previous study among food allergic children aged 8 to 12 years and their parents suggest that parents report better HRQL than children. ${ }^{119}$ To date, child-reported food allergy specific HRQL questionnaires are available for children aged 8 years and older. Instruments to assess HRQL in younger children are needed, especially now that prevention strategies and immunotherapy target this age group. ${ }^{73}$ The assessment of patient-reported outcomes before and after immunotherapy is gaining increasing importance and is critical in the evaluation of the benefits and risks of immunotherapy. ${ }^{77}$ 


\section{References}

1. Dyer AA, Rivkina V, Perumal D, Smeltzer BM, Smith BM, Gupta RS. Epidemiology of childhood peanut allergy. Allergy asthma Proc. 2015;36(1):58-64.

2. Grabenhenrich LB, Dölle S, Moneret-Vautrin A, et al. Anaphylaxis in children and adolescents: The European Anaphylaxis Registry. J Allergy Clin Immunol. 2016;137(4):1128-1137.e1.

3. Horuihane JO., Roberts SA., Warner JO. Resolution of peanut allergy: case-control study. $\mathrm{Br} \mathrm{Med}$ J. 1998;316(7140):1267-1271.

4. Cummings a. J, Knibb RC, King RM, Lucas JS, Lucas JS, Wellcome SH. The psychosocial impact of food allergy and food hypersensitivity in children, adolescents and their families: A review. Allergy Eur J Allergy Clin Immunol. 2010;65(8):933-945.

5. Primeau MN, Kagan $R$, Joseph $L$, et al. The psychological burden of peanut allergy as perceived by adults with peanut allergy and the parents of peanut-allergic children. Clin Exp Allergy. 2000;30(8):1135-1143.

6. Gupta R, Holdford D, Bilaver L, Dyer A, Holl JL, Meltzer D. The Economic Impact of Childhood Food Allergy in the United States. JAMA Pediatr. 2016;167(11):1026-1031.

7. Nwaru BI, Hickstein L, Panesar SS, Roberts G, Muraro A, Sheikh A. Prevalence of common food allergies in Europe: A systematic review and meta-analysis. Allergy. 2014;69(8):992-1007.

8. Nederlandse Vereniging voor Allergologie. Richtlijn Voedselprovocatie. https://www.nvk.nl/Portals/0/richtlijnen/Voedselprovocatie/Richtlijn Voedselprovocatie.pdf. Published 2015. Accessed December 9, 2019.

9. Muraro A, Werfel T, Hoffmann-Sommergruber K, et al. EAACI Food Allergy and Anaphylaxis Guidelines: Diagnosis and management of food allergy. Allergy. 2014;69(8):1008-1025.

10. Krogulska A, Id O. Peanut allergy diagnosis: moving from basic to more elegant testing. Pediatr Allergy Immunol. 2020.

11. van Erp FC, Knol EF, Pontoppidan B, Meijer Y, van der Ent CK, Knulst AC. The IgE and basophil responses to Ara $\mathrm{h} 2$ and Ara $\mathrm{h} 6$ are good predictors of peanut allergy in children. $J$ Allergy Clin Immunol. 2017;139(1):358-360.e8.

12. Preece $K$, Ang $M$, Barker $D$, et al. Regional centres conduct food challenges with outcomes equivalent to a tertiary pediatric hospital. Pediatr Allergy Immunol. 2019;30(7):764-767.

13. Asarnoj A, Glaumann S, Elfstrom $L$, et al. Anaphylaxis to peanut in a patient predominantly sensitized to Ara h 6. Int Arch Allergy Immunol. 2012;159(2):209-212.

14. Leo SH, Dean JM, Jung B, Kuzeljevic B, Chan ES. Utility of Ara h 2 sIgE levels to predict peanut allergy in Canadian children. J Allergy Clin Immunol Pr. 2015;3(6):968-969.

15. Zambrano Ibarra G, Fuentes Aparicio V, Infante Herrero S, Blanca M, Zapatero Remon L. Peanut Allergy in Spanish Children: Comparative Profile of Peanut Allergy versus Tolerance. Int Arch Allergy Immunol. 2019;178(4):370-376.

16. Preece $\mathrm{K}$, Bhatia $\mathrm{R}$, Belcher J, et al. The fraction of exhaled nitric oxide improves prediction of clinical allergic reaction to peanut challenge in children. Clin Exp Allergy. 2014;44(3):371-380.

17. Ballmer-Weber BK, Lidholm J, Fernandez-Rivas $M$, et al. IgE recognition patterns in peanut allergy are age dependent: perspectives of the EuroPrevall study. Allergy. 2015;70(4):391-407.

18. Schots $\mathrm{M}, \mathrm{Mol} \mathrm{AC}$ De, Vermeer $\mathrm{HJ}$, et al. Is Ara $\mathrm{h} 2$ indeed the best predictor for peanut allergy in Dutch children? Diagnosis. 2016;0(0):2-6.

19. Klemans RJB, Broekman HCHP, Knol EF, et al. Ara $\mathrm{h} 2$ is the best predictor for peanut allergy in adults. J Allergy Clin Immunol Pract. 2013;1(6):632-638.e1.

20. Beyer K, Grabenhenrich L, Härtl M, et al. Predictive values of component-specific IgE for the outcome of peanut and hazelnut food challenges in children. Allergy Eur J Allergy Clin Immunol. 2015;70(1):90-98.

21. Kukkonen AK, Pelkonen AS, Makinen-Kiljunen S, Voutilainen $\mathrm{H}$, Makela MJ. Ara h 2 and Ara 6 are the best predictors of severe peanut allergy: a double-blind placebo-controlled study. Allergy. 2015;70(10):1239-1245.

22. Valcour A, Jones JE, Lidholm J, Borres MP, Hamilton RG. Sensitization profiles to peanut 
allergens across the United States. Ann Allergy, Asthma Immunol. 2017;119(3):262-266.e1.

23. Schmitz R, Ellert U, Kalcklösch M, Dahm S, Thamm M. Patterns of sensitization to inhalant and food allergens - Findings from the German health interview and examination survey for children and adolescents. Int Arch Allergy Immunol. 2013;162(3):263-270.

24. Bilaver LA, Chadha AS, Doshi P, Dwyer LO, Gupta RS. Economic burden of food allergy A systematic review. Ann Allergy, Asthma Immunol. 2019;122(4):373-380.e1.

25. Nunes C, Pereira AM, Morais-almeida M. Asthma costs and social impact. Asthma Res Pract. 2017:1-11.

26. Chung J, Simpson EL. The socioeconomics of atopic dermatitis. Ann Allergy, Asthma Immunol. 2019;122(4):360-366.

27. Cardell LO, Olsson P, Andersson M, et al. TOTALL: High cost of allergic rhinitis - A national Swedish population-based questionnaire study. npj Prim Care Respir Med. 2016;26(March 2015):1-5.

28. Dalal AA, Stanford R, Henry H, Borah B. Economic burden of rhinitis in managed care: A retrospective claims data analysis. Ann Allergy, Asthma Immunol. 2008;101(1):23-29.

29. Nurmagambetov T, Kuwahara R, Garbe P. The economic burden of asthma in the United States, 2008-2013. Ann Am Thorac Soc. 2018;15(3):348-356.

30. Zink A, Arents B, Fink-Wagner A, et al. Out-of-pocket costs for individuals with atopic Eczema: A cross-sectional study in nine European countries. Acta Derm Venereol. 2019;99(3):263-267.

31. Karaca-Mandic P., Jena AB., Joyce GF., Goldman DP. Out-of-pocket medication costs, medication utilization, and use of healthcare services among children with asthma. JAMA. 2012;307(12):1284-1291.

32. Law AW, Reed SD, Sundy JS, Schulman KA. Direct costs of allergic rhinitis in the United States: Estimates from the 1996 Medical Expenditure Panel Survey. I Allergy Clin Immunol. 2003;111(2):296-300.

33. Le T-M, van Hoffen E, Kummeling I, et al. Food allergy in the Netherlands: differences in clinical severity, causative foods, sensitization and DBPCFC between community and outpatients. Clin Transl Allergy. 2015;5:8.

34. Statline. Bevolking; geslacht, leeftijd en burgelijke staat, 1 januari. Centraal Bureau voor de Statistiek.

35. Kunst NR, Lindvik H, Carlsen K-H, Håland G, Jørgensen E, Lødrup Carlsen KC. Cost-effectiveness of diagnostic algorithms for peanut allergy in children. J Allergy Clin Immunol. 2019;143(3):1243-1246.

36. Spergel AKR, Togias A. Observational human studies in allergic diseases: design concepts and highlights of recent National Institute of Allergy and Infectious Diseases-funded research. Curr Opin Allergy Clin Immunol. 2020:1-7.

37. Capucilli P, Wang KY, Spergel JM. Food reactions during avoidance: focus on peanut. Ann Allergy, Asthma Immunol. 2020.

38. Blom WM, Michelsen-Huisman $A D$, van Os-Medendorp $H$, et al. Accidental food allergy reactions: Products and undeclared ingredients. J Allergy Clin Immunol. 2018;142(3):865-875.

39. Warren $\mathrm{CM}$, Dyer $\mathrm{AA}$, Otto $\mathrm{AK}$, et al. Food Allergy-Related Risk-Taking and Management Behaviors Among Adolescents and Young Adults. J Allergy Clin Immunol Pract. 2017;5(2):381390.e13.

40. Warren CM, Zaslavsky JM, Kan K, Spergel JM, Gupta RS. Epinephrine auto-injector carriage and use practices among US children, adolescents, and adults. Ann Allergy, Asthma Immunol. 2018;121(4):479-491.

41. Grabenhenrich LB, Dölle S, Ruëff F, et al. Epinephrine in Severe Allergic Reactions: The European Anaphylaxis Register. J Allergy Clin Immunol Pract. 2018;6(6):1898-1906.e1.

42. Noimark L, Wales J, Du Toit $G$, et al. The use of adrenaline autoinjectors by children and teenagers. Clin Exp Allergy. 2012;42(2):284-292.

43. Sicherer SH, Simons FER, Mahr TA, et al. Epinephrine for first-aid management of anaphylaxis. Pediatrics. 2017;139(3).

44. Kaur N., McCrossin T., Gunasekera H. Improving anaphylaxis management by health care 
professional education and practical skills training in a regional centre. $J$ Paediatr Child Health. 2017;53(10):1029.

45. Cohen MB, Saunders SS, Wise SK, Nassif S, Platt MP. Pitfalls in the use of epinephrine for anaphylaxis: patient and provider opportunities for improvement. Int Forum Allergy Rhinol. 2017;7(3):276-286.

46. Eigenmann PA, Caubet JC, Zamora SA. Continuing food-avoidance diets after negative food challenges. Pediatr Allergy Immunol. 2006:601-605.

47. Gau J, Wang J. Rate of food introduction after a negative oral food challenge in the pediatric population. J Allergy Clin Immunol Pract. 2017;5(2):475-476.

48. van Erp FC, Boot J, Knulst AC, Pasmans SGM, van der Ent CK, Meijer Y. Reintroduction failure after negative peanut challenges in children. Pediatr Allergy Immunol. 2014;25(6):580-585.

49. van der Valk JPM, van Wijk RG, Vergouwe $Y$, de Jong NW. Failure of introduction of food allergens after negative oral food challenge tests in children. Eur J Pediatr. 2015;174(8):10931099.

50. Sharp WG, Jaquess DL, Morton JF, Herzinger C V. Pediatric Feeding Disorders: A Quantitative Synthesis of Treatment Outcomes. Clin Child Fam Psychol Rev. 2010;13(4):348-365.

51. Hemmings $O$, Kwok M, McKendry R, Santos AF. Basophil Activation Test: Old and New Applications in Allergy. Curr Allergy Asthma Rep. 2018;18(12).

52. Bahri R, Custovic A, Korosec $\mathrm{P}$, et al. Mast cell activation test in the diagnosis of allergic disease and anaphylaxis. J Allergy Clin Immunol. 2018;142(2):485-496.e16.

53. Lin J, Bruni FM, Fu Z, et al. A bioinformatics approach to identify patients with symptomatic peanut allergy using peptide microarray immunoassay. J Allergy Clin Immunol. 2012;129(5):1321-1328.e5.

54. Flinterman $A E$, Knol EF, Lencer DA, et al. Peanut epitopes for IgE and IgG4 in peanut-sensitized children in relation to severity of peanut allergy. J Allergy Clin Immunol. 2008;121(3):737743.e10.

55. Ehlers AM, Blankestijn MA, Knulst AC, Klinge M, Otten HG. Can alternative epitope mapping approaches increase the impact of B-cell epitopes in food allergy diagnostics? Clin Exp Allergy. 2019;49(1):17-26.

56. Dahl A. Pollen Lipids Can Play a Role in Allergic Airway Inflammation. Front Immunol. 2018;9(December):2816.

57. Del Moral MG, Martínez-Naves E. The role of lipids in development of allergic responses. Immune Netw. 2017;17(3):133-143.

58. Turner PJ, Baumert JL, Beyer K, et al. Can we identify patients at risk of life-threatening allergic reactions to food? 2016.

59. Pettersson ME, Koppelman GH, Flokstra-de Blok BMJ, Kollen BJ, Dubois AEJ. Prediction of the severity of allergic reactions to foods. Allergy Eur J Allergy Clin Immunol. 2018;73(7):1532-1540.

60. Ruiter B, Smith NP, Monian B, et al. Expansion of the CD4+ effector T-cell repertoire characterizes peanut-allergic patients with heightened clinical sensitivity. J Allergy Clin Immunol. 2020;145(1):270-282.

61. Do AN, Watson CT, Cohain AT, et al. Dual transcriptomic and epigenomic study of reaction severity in peanut allergic children. J Allergy Clin Immunol. 2019.

62. Hourihane JOB, Allen KJ, Shreffler WG, et al. Peanut Allergen Threshold Study (PATS): Novel single-dose oral food challenge study to validate eliciting doses in children with peanut allergy. J Allergy Clin Immunol. 2017;139(5):1583-1590.

63. Graham F, Caubet J, Eigenmann PA. Can my child with IgE-mediated peanut allergy introduce foods labeled with "may contain traces"? Pediatr Allergy Immunol. 2020.

64. Du Toit $G$, Roberts $G$, Sayre $P H$, et al. Randomized trial of peanut consumption in infants at risk for peanut allergy. N Engl J Med. 2015;372(9):803-813.

65. Togias A, Cooper SF, Acebal ML, et al. Addendum guidelines for the prevention of peanut allergy in the United States: Report of the National Institute of Allergy and Infectious Diseasessponsored expert panel. J Allergy Clin Immunol. 2017;139(1):29-44.

66. Turner PJ, Campbell DE, Boyle RJ, Levin ME. Primary Prevention of Food Allergy: Translating 
Evidence from Clinical Trials to Population-Based Recommendations. I Allergy Clin Immunol Pract. 2018;6(2):367-375.

67. Stadermann M, Meijer Y, Klok T. Vroege introductie van hoog-allergene voeding bij zuigelingen ter preventie van voedselallergie. NVK-standpunten.

68. Trendelenburg V, Rohrbach A, Schulz G, Schwarz V, Beyer K. Molecular sIgE profile in infants and young children with peanut sensitization and eczema. Allergo J Int. 2014;23(5):152-157.

69. Soller L, Abrams EM, Carr S, et al. First Real-World Safety Analysis of Preschool Peanut Oral Immunotherapy. J Allergy Clin Immunol Pract. 2019;7(8):2759-2767.e5.

70. Jones SM, Sicherer SH, Burks AW, et al. Epicutaneous immunotherapy for the treatment of peanut allergy in children and young adults. J Allergy Clin Immunol. 2017;139(4):1242-1252.e9.

71. Palforzia. U.S. Food \& Drug Administration.

72. Pajno GB, Fernandez-Rivas $M$, Arasi S, et al. EAACI Guidelines on allergen immunotherapy: IgEmediated food allergy. Allergy Eur J Allergy Clin Immunol. 2018;73(4):799-815.

73. Vickery BP, Berglund JP, Burk CM, et al. Early oral immunotherapy in peanut-allergic preschool children is safe and highly effective. J Allergy Clin Immunol. August 2016.

74. Hsiao K-C, Ponsonby A-L, Axelrad C, et al. Long-term clinical and immunological effects of probiotic and peanut oral immunotherapy after treatment cessation: 4-year follow-up of a randomised, double-blind, placebo-controlled trial. Lancet Child Adolesc Heal. 2017;4642(17):19.

75. Dunlop JH, Keet CA. Goals and motivations of families pursuing oral immunotherapy for food allergy. J Allergy Clin Immunol Pract. 2019;7(2):662-663.e18.

76. DunnGalvin A, Wen CC, Laubach $S$, et al. Profiling families enrolled in food allergy immunotherapy studies. Pediatrics. 2009;124(3).

77. Chu DK, Wood RA, French S, et al. Oral immunotherapy for peanut allergy (PACE): a systematic review and meta-analysis of efficacy and safety. Lancet. 2019;393(10187):2222-2232.

78. Yoon PW, Scheuner MT, Peterson-Oehlke KL, Gwinn M, Faucett A, Khoury MJ. Can family history be used as a tool for public health and preventive medicine? Genet Med. 2002;4(4):304310.

79. Gupta J, Johansson E, Bernstein JA, et al. Resolving the Etiology of Atopic Disorders by Genetic Analysis of Racial Ancestry. J Allergy Clin Immunol. 2016;138(3):676-699.

80. Elmose C, Thomsen SF. Twin Studies of Atopic Dermatitis: Interpretations and Applications in the Filaggrin Era. J Allergy. 2015;2015:1-7.

81. Ravn NH, Halling AS, Berkowitz AG, et al. How does parental history of atopic disease predict the risk of atopic dermatitis in a child? A systematic review and meta-analysis. J Allergy Clin Immunol. 2020:36-44.

82. van Beijsterveldt CEM, Boomsma DI. Genetics of parentally reported asthma, eczema and rhinitis in 5-yr-old twins. Eur Respir J. 2007;29(3):516-521.

83. Lichtenstein $\mathrm{P}$, Svartengren M. Genes, environments, and sex: Factors of importance in atopic diseases in 7-9-year-old Swedish twins. Allergy Eur J Allergy Clin Immunol. 1997;52(11):10791086.

84. Reust CE. Evaluation of primary immunodeficiency disease in children. Am Fam Physician. 2013;87(11):773-778.

85. Super $M$, Barnes $R$, Greig D. Failure to refer for testing for cystic fibrosis. Bmj. 2001;322(7282):310-311.

86. Barbato $\mathrm{A}$, Frischer $\mathrm{T}$, Kuehni $\mathrm{CE}$, et al. Primary ciliary dyskinesia: $\mathrm{A}$ consensus statement on diagnostic and treatment approaches in children. Eur Respir J. 2009;34(6):1264-1276.

87. Tarini BA, McInerney JD. Family history in primary care pediatrics. Pediatrics. 2013;132(SUPPL.3).

88. Kingsley C, Patel S. Patient-reported outcome measures and patient-reported experience measures. BJA Educ. 2017;17(4):137-144.

89. Flokstra-De Blok BMJ, Dubois AEJ, Vlieg-Boerstra BJ et al. Health-related quality of life of food allergic patients: comparison with the general population and other diseases. Allergy. 2010;65(2):238-44.

90. van der Velde JL, Dubois AEJ, Flokstra-de Blok BMJ. Food allergy and quality of life: what have 
we learned? Curr Allergy Asthma Rep. 2013;13(6):651-661.

91. Henegouwen B. Social position of adolescents with chronic digestive disorders. 2002;(5):543549.

92. Stucky BD, Sherbourne CD, Edelen MO, Eberhart NK. Understanding asthma-specific quality of life: Moving beyond asthma symptoms and severity. Eur Respir J. 2015;46(3):680-687.

93. Jonsson M, Bergström A, Egmar AC, Hedlin G, Lind T, Kull I. Asthma during adolescence impairs health-related quality of life. J Allergy Clin Immunol Pract. 2016;4(1):144-146.e2.

94. Herbert LJ, Dahlquist LM, Bollinger ME. Maternal intolerance of uncertainty, anxiety, and adherence with food challenge referrals. J Health Psychol. 2013;18(9):1209-1219.

95. Zijlstra WT, Flinterman AE, Soeters $L$, et al. Parental anxiety before and after food challenges in children with suspected peanut and hazelnut allergy. Pediatr Allergy Immunol. 2010;21:439-445.

96. Bird JA, Leonard S, Groetch $\mathrm{M}$, et al. Conducting an Oral Food Challenge: An Update to the 2009 Adverse Reactions to Foods Committee Work Group Report. J Allergy Clin Immunol Pract. 2020;8(1):75-90.e17.

97. Knibb RC, Semper H. Impact of suspected food allergy on emotional distress and family life of parents prior to allergy diagnosis. Pediatr Allergy Immunol. 2013;24(8):798-803.

98. Boyle RJ, Umasunthar T, Smith JG, et al. A brief psychological intervention for mothers of children with food allergy can change risk perception and reduce anxiety: Outcomes of a randomized controlled trial. Clin Exp Allergy. 2017;47(10):1309-1317.

99. Dantzer JA, Keet CA. Anxiety associated with food allergy in in adults and adolescents: An analysis of data from the National Health and Nutrition Examination Survey (NHANES) 20072010. J Allergy Clin Immunol Pract. 2020:2007-2010.

100. Global Strategy for Asthma Management and Prevention. Global Initiative for Asthma.

101. Bateman ED, Bousquet J, Keech ML, Busse WW, Clark TJH, Pedersen SE. The correlation between asthma control and health status: The GOAL study. Eur Respir J. 2007;29(1):59-63.

102. Chen H, Gould MK, Blanc PD, et al. Asthma control, severity, and quality of life: Quantifying the effect of uncontrolled disease. J Allergy Clin Immunol. 2007;120(2):396-402.

103. Jansson SA, Axelsson M, Hedman L, Leander M, Stridsman C, Rönmark E. Subjects with wellcontrolled asthma have similar health-related quality of life as subjects without asthma. Respir Med. 2016;120:64-69.

104. Yaghoubi M, Adibi A, Safari A, FitzGerald JM, Sadatsafavi M. The projected economic and health burden of uncontrolled asthma in the United States. Am J Respir Crit Care Med. 2019;200(9):1102-1112.

105. Rabe KF, Vermeire PA, Soriano JB, Maier WC. Clinical management of asthma in 1999: The Asthma Insights and Reality in Europe (AIRE) study. Eur Respir J. 2000;16(5):802-807.

106. Matsunaga K, Hamada K, Oishi K, Yano M, Yamaji Y, Hirano T. Factors Associated with Physician-Patient Discordance in the Perception of Asthma Control. J Allergy Clin Immunol Pract. 2019;7(8):2634-2641.

107. Nathan RA, Sorkness CA, Kosinski M, et al. Development of the Asthma Control Test: A survey for assessing asthma control. J Allergy Clin Immunol. 2004;113:59-65.

108. Liu AH, Zeiger R, Sorkness $\mathrm{C}$, et al. Development and cross-sectional validation of the Childhood Asthma Control Test. J Allergy Clin Immunol. 2007;119:817-825.

109. Global Initiative for Asthma (GINA). Global Strategy for Asthma Management and Prevention. Available from: https://ginasthma.org (2018). Accessed Aug 8, 2018.

110. Chipps BE, Haselkorn T, Rosén K, Mink DR, Trzaskoma BL, Luskin AT. Asthma Exacerbations and Triggers in Children in TENOR: Impact on Quality of Life. J Allergy Clin Immunol Pract. 2018;6(1):169-176.e2.

111. Luskin AT, Chipps BE, Rasouliyan L, Miller DP, Haselkorn T, Dorenbaum A. Impact of Asthma Exacerbations and Asthma Triggers on Asthma-related Quality of Life in Patients with Severe or Difficult-to-Treat Asthma. J Allergy Clin Immunol Pract. 2014;2(5):544-552.

112. Ritz T, Wittchen HU, Klotsche J, Mühlig S, Riedel O. Asthma trigger reports are associated with low quality of life, exacerbations, and emergency treatments. Ann Am Thorac Soc. 2016;13(2):204-211. 
113. Peterson MGE, Gaeta TJ, Birkhahn RH, Fernández JL, Mancuso C a. History of symptom triggers in patients presenting to the emergency department for asthma. J Asthma. 2012;49(6):629-636.

114. Gautier C, Charpin D. Environmental triggers and avoidance in the management of asthma. J Asthma Allergy. 2017;10:47-56.

115. Huber $M$, André Knottnerus J, Green L, et al. How should we define health? BMJ. 2011;343(7817):1-3.

116. The Lancet. What is health? The ability to adapt. Lancet. 2009;373(9666):781.

117. Saracci R. The World Health Organisation needs to reconsider its definition of health. BMJ. 1997;314(7091):1409-1410.

118. Huber $M$, Van Vliet $M$, Giezenberg $M$, et al. Towards a "patient-centred" operationalisation of the new dynamic concept of health: A mixed methods study. BMJ Open. 2016;6(1):1-11.

119. Van der Velde JL, Flokstra-de Blok BMJ, Dunngalvin a., Hourihane JOB, Duiverman EJ, Dubois AE. Parents report better health-related quality of life for their food-allergic children than children themselves. Clin Exp Allergy. 2011;41(10):1431-1439. 


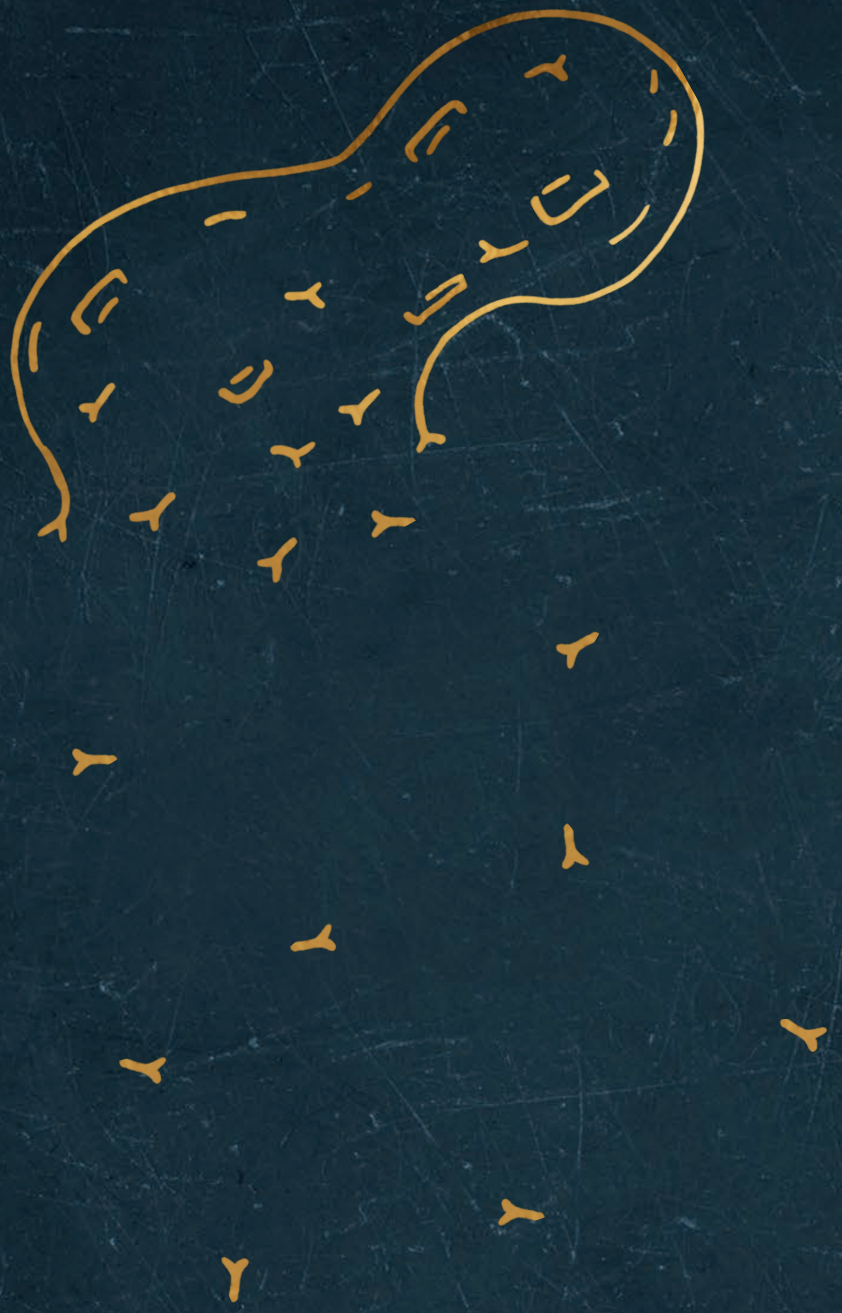

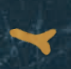

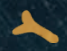




\section{Chapter 10}

Nederlandse samenvatting 
Voedselallergie, astma, hooikoorts en eczeem komen vaak voor bij kinderen. Deze ziekten zijn gebaseerd op een niet zinvolle en zelfs schadelijke reactie van ons afweersysteem en worden atopische ziekten genoemd.

Het begrip 'atopie' is afgeleid van het Griekse woord atopia en betekent 'ongewoonheid'. Atopie wijst op de aanleg van een persoon om antistoffen (van het type IgE) te maken. Deze antistoffen zijn gericht tegen 'onschuldige' eiwitten uit de omgeving, zoals de eiwitten van voedsel (bijvoorbeeld pinda of noten), huisstofmijt, pollen of gras. Wanneer deze antistoffen worden aangemaakt kan dat leiden tot verschillende atopische ziekten, namelijk voedselallergie, astma, hooikoorts en eczeem. Kinderen hebben vaak meerdere atopische ziekten.

In dit proefschrift wordt onderzocht hoe we atopische ziekten beter kunnen vaststellen (diagnostiek). De focus ligt hierbij op pinda allergie. Daarnaast richten we ons op de kwaliteit van leven van kinderen met atopische ziekten.

\section{Diagnostiek van atopische ziekten}

In de zorg voor patiënten zijn drie hoekstenen te onderscheiden. Allereerst het vaststellen van de ziekte (diagnose), vervolgens het inschatten van het vermoedelijke beloop van de ziekte (prognose) en ten derde het voorstellen van een behandeling (therapie) voor de ziekte. Het vaststellen van ziekten vindt plaats door het in kaart brengen van de klachten, lichamelijk onderzoek en, indien nodig, aanvullend diagnostisch onderzoek. Aanvullend diagnostisch onderzoek van atopische ziekten is bijvoorbeeld de bepaling van specifieke antistoffen (sIgE) in het bloed of een huidpriktest, een voedselprovocatietest of longfunctieonderzoek. Het stellen van de juiste diagnose is belangrijk om patiënten goed te adviseren en, voor zover mogelijk, te behandelen.

\section{Pinda allergie}

Pinda allergie is één van de meest voorkomende voedselallergieën bij kinderen. Een pinda allergie is meestal blijvend en kenmerkt zich vaak door ernstige klachten. Een patiënt met een pinda allergie ontwikkelt klachten binnen enkele minuten tot twee uur na het eten van (een product met) pinda. De klachten kunnen uiteenlopen van mild, waarbij u kunt denken aan een tinteling in de mond, rode huid, galbulten, jeuk, rode ogen of niezen, tot ernstig, wat zich uit in bijvoorbeeld zwelling van de tong en keel, benauwdheid of shock.

De huidige 'gouden standaard' om een pinda allergie aan te tonen is een 2-daagse voedselprovocatie. Deze voedselprovocatie vindt plaats in het ziekenhuis en bestaat uit het eten van een cakeje, waarin op één dag pinda is verwerkt (verum- 
dag) en op de andere dag niet (placebo-dag). Een voedselprovocatie geeft duidelijkheid over de diagnose. Als er inderdaad sprake is van een voedselallergie dan kan de voedselprovocatie ook helpen om meer te weten te komen over hoeveel pinda een allergische reactie veroorzaakt en hoe ernstig deze reactie vervolgens is. Er kleven echter ook enkele nadelen aan de test. Deze is namelijk behoorlijk belastend en de klachten van een voedselprovocatie zijn soms lastig te interpreteren. Daarnaast is de test kostbaar in financieel opzicht. Eén van de doelen van het onderzoek beschreven in dit proefschrift is om het aantal 2-daagse voedselprovocaties te verminderen bij kinderen (hoofdstuk 2) en bij volwassenen (hoofdstuk 3).

Een patiënt met een pinda allergie maakt specifieke antistoffen (sIgE) aan tegen pinda. Als de patiënt pinda eet kan het pinda-allergeen binden aan de sIgE antistoffen, waarna er stoffen vrijkomen, die klachten veroorzaken bij een patiënt met pinda allergie. De hoeveelheid sIgE tegen pinda kan gemeten worden in het bloed, zowel sIgE tegen een mix van pinda eiwitten (pinda extract) als tegen specifieke pinda eiwitten (pinda componenten). De hoeveelheid sIgE tegen pinda componenten, zoals Ara $\mathrm{h} 2$, voorspelt pinda allergie beter dan de hoeveelheid sIgE tegen pinda extract. De hoogte van het sIgE tegen Ara $\mathrm{h} 2$ in het bloed kan gebruikt worden om de kans op een pinda allergie in te schatten. Er wordt dan gebruik gemaakt van afkappunten. Uit eerder onderzoek in ons ziekenhuis is gebleken dat alle kinderen met een Ara h 2 waarde onder het lage afkappunt geen pinda allergie hadden en alle kinderen met een Ara $\mathrm{h} 2$ waarde boven het hoge afkappunt wel. Met deze afkappunten van Ara h 2 kon de uitslag van een voedselprovocatie voor pinda bij $62 \%$ van de kinderen juist voorspeld worden.

In hoofdstuk 2 hebben we een beslisregel op basis van deze afkappunten van sIgE tegen Ara h 2 gemaakt. We hebben de beslisregel ingevoerd in vier ziekenhuizen, één academisch ziekenhuis en drie streekziekenhuizen, bij kinderen van 3.5 tot 18 jaar met een verdenking op een pinda allergie. Kinderen met een Ara h 2 waarde onder het afkappunt waarbij zeker is dat ze niet pinda allergisch zijn, mochten thuis pinda gaan eten. De kinderen met een waarde boven het afkappunt waarbij zeker is dat ze wel pinda allergisch zijn kregen een 1-daagse open voedselprovocatie. De kinderen met een Ara h 2 waarde tussen de afkappunten (een twijfelachtige Ara h 2 waarde) kregen wel een 2-daagse voedselprovocatie.

Dit onderzoek laat zien dat de beslisregel pinda allergie goed voorspelt (89-97\%) en uitsluit (100\%). Daarnaast was het gebruik van de beslisregel veilig, aangezien alle kinderen die thuis pinda mochten eten geen klachten kregen. De zorg werd bij twee derde van de kinderen uitgevoerd volgens de beslisregel. De voornaamste redenen om van de beslisregel af te wijken waren angst voor een allergische reactie of een recente allergische reactie na het eten van pinda. 
Naast de geringere belasting voor de patiënten, leidde de invoer van de beslisregel tevens tot lagere kosten ( $€ 320$ tot $€ 636$ per patiënt per jaar) vergeleken met de diagnostiek van pinda allergie volgens de huidige Nederlandse richtlijn. Het invoeren van de beslisregel in heel Nederland zou zodoende tot een besparing van $€ 767.101$ tot $€ 1.526 .688$ per jaar kunnen leiden.

Bij eerder onderzoek in ons ziekenhuis werd een afkappunt van sIgE tegen Ara h 2 gevonden waarmee pinda allergie met $100 \%$ zekerheid kon worden aangetoond bij volwassenen. In hoofdstuk $\mathbf{3}$ is dit afkappunt gecontroleerd in een vervolgstudie. Het afkappunt klopte en voorspelde pinda allergie correct bij één derde van de volwassenen met een verdenking op pinda allergie. Daarnaast is onderzocht of andere pinda componenten (Ara h 1, 3, 6 en 8) een toevoegde waarde hebben bij het vaststellen van een pinda allergie. Van deze pinda componenten voorspelde Ara h 6 pinda allergie even goed als Ara h 2. De andere pindacomponenten speelden geen rol bij het vaststellen van een pinda allergie. Aangezien sIgE tegen Ara h 2 en Ara h 6 sterk met elkaar samenhangen, en het afkappunt van sIgE tegen Ara h 2 nu gecontroleerd is, hebben we net als bij kinderen een beslisregel op basis van sIgE tegen Ara h 2 ontwikkeld. Bij een derde van de volwassenen met een verdenking op pinda allergie en een Ara $h 2$ waarde boven het gecontroleerde afkappunt zou de 2-daagse dubbelblinde voedselprovocatie vervangen kunnen worden door een 1-daagse open voedselprovocatie.

We hebben 83 kinderen en ouders een vragenlijst gestuurd 3 jaar na een voedselprovocatie voor pinda om te vragen hoe het met hen gaat. Dit waren zowel kinderen bij wie een pinda allergie was aangetoond, als kinderen bij wie een pinda allergie was uitgesloten. In hoofdstuk 4 beschrijven we de resultaten van dit onderzoek. De kinderen bij wie een pinda allergie was vastgesteld kregen na de voedselprovocatie het advies om pinda te vermijden. Ondanks deze adviezen rapporteerde toch $41 \%$ van de kinderen een allergische reactie door pinda tijdens de 3 jaar na de voedselprovocatie. Kinderen met ernstige allergische reacties gebruikten helaas geen adrenalinepen. Een derde van de kinderen bij wie een pinda allergie was uitgesloten lukte het niet om pinda te gaan of blijven eten. Deze resultaten suggereren dat het belangrijk is om kinderen na een voedselprovocatie goed te begeleiden, om allergische reacties te voorkomen of beter te behandelen (na een voedselprovocatie waarin pinda allergie is aangetoond) en om pinda niet langer te vermijden (na een voedselprovocatie waarin pinda allergie is uitgesloten). 


\section{Atopische ziekten en luchtweginfecties}

De resultaten van hoofdstuk $\mathbf{5}$ laten zien dat de factoren die samen hangen met atopische ziekten verschillen van de factoren die samen hangen met herhaaldelijke luchtweginfecties. De aanwezigheid van één of meerdere atopische ziekten was minder waarschijnlijk bij kinderen met een jonge leeftijd ( $\leq 4$ jaar) of een laag geboortegewicht, bij meisjes en bij kinderen met een hond als huisdier. De aanwezigheid van herhaaldelijke luchtweginfecties was juist waarschijnlijker bij kinderen $\leq 4$ jaar vergeleken met oudere kinderen, en als kinderen naar de crèche gingen.

Kinderen met ouders, broertjes en/of zusjes met astma hadden vaker astma. Hetzelfde gold voor hooikoorts, eczeem en herhaaldelijke luchtweginfecties. Bij kinderen die verdacht worden van een atopische ziekte of herhaaldelijke luchtweginfecties raden we dan ook aan om goed uit te vragen of familieleden ook last hebben van deze ziekten. Op deze manier kan de arts de kans op atopische ziekten en herhaaldelijke luchtweginfecties beter inschatten.

\section{Impact van atopische ziekten op de kwaliteit van leven}

Atopische ziekten hebben een negatieve invloed op de kwaliteit van leven. Kinderen met voedselallergie moeten opletten met wat ze eten en het is dan ook vaak lastiger om bijvoorbeeld spontaan buiten de deur te eten of traktaties op school of werk aan te nemen. Daarnaast zijn kinderen en hun ouders vaak bang voor een allergische reactie. Eerder onderzoek heeft laten zien dat kinderen met astma nog meer beperkingen in hun kwaliteit van leven ervaren vergeleken met kinderen met een voedselallergie. Eén van de belangrijkste factoren die de kwaliteit van leven van kinderen met astma beïnvloedt is de mate waarin de astmaklachten gecontroleerd zijn. Kinderen met astma kunnen last hebben van een piepende ademhaling, hoesten of kortademigheid bijvoorbeeld tijdens het sporten, als ze spelen met dieren of 's nachts. Hoe meer klachten van astma (ongecontroleerd astma), des te meer beperkingen in de kwaliteit van leven kinderen ervaren.

\section{Pinda allergie}

In hoofdstuk 6 is de literatuur samengevat over de invloed van de diagnostiek naar voedselallergie op de kwaliteit van leven. Aangetoond werd dat de kwaliteit van leven verbeterde na voedselprovocaties, zowel wanneer een voedselallergie werd aangetoond als wanneer een voedselallergie werd uitgesloten.

Vervolgens hebben we in hoofdstuk 2 de angst van ouders van kinderen met een verdenking op een pinda allergie gemeten bij start van de studie, na een telefonische afspraak om de sIgE tegen Ara h 2 uitslag te bespreken en na 6 
maanden follow-up. Het onderzoek laat zien dat de angst van ouders afnam na het horen van een negatieve Ara h 2 uitslag (geen pinda allergie) en na het bespreken van een negatieve of positieve uitslag van de voedselprovocatietest. De resultaten van hoofdstuk 2 en 6 laten zien dat een duidelijke diagnose van pinda allergie (zowel aantonen als uitsluiten) de kwaliteit van leven verbetert en de angst van ouders vermindert.

In hoofdstuk 4 is de kwaliteit van leven van ouders van kinderen met een verdenking op een pinda allergie gemeten bij start van de studie (voor een voedselprovocatie) en na 3-jaar follow-up. De kwaliteit van leven bleek sterk te verbeteren bij ouders van kinderen met een negatieve uitslag van de voedselprovocatie (geen pinda allergie). De kwaliteit van leven bleek nog veel meer te verbeteren als deze kinderen na de test gewoon pinda gingen eten, in vergelijking tot kinderen die nog steeds geen pinda aten. Deze resultaten benadrukken het belang van het gaan eten en het niet meer vermijden van pinda na een negatieve voedselprovocatie.

\section{Astma}

Hoofdstuk 7 laat zien dat ongecontroleerd astma (veel last van hoesten en piepen) vaak voorkomt bij kinderen die al bekend zijn met astma en hiervoor naar het ziekenhuis komen, maar ook bij kinderen met astma die voor een andere atopische ziekte naar het ziekenhuis komen. Kinderen met astma in de familie (bij ouders, broertjes en/of zusjes) hadden een grotere kans om veel last te hebben van astmaklachten. Ook kinderen met herhaaldelijke onderste of bovenste luchtweginfecties en astma als reden om naar het ziekenhuis te komen hadden vaker ongecontroleerd astma. Hieruit concluderen we dat het belangrijk is om te meten hoe goed astma onder controle is, zowel bij de kinderen die worden verwezen vanwege astma als bij de kinderen die worden verwezen vanwege een andere atopische ziekte.

Hoe goed astma onder controle is, wordt beïnvloed en uitgelokt door allerlei factoren. Astmaklachten kunnen bijvoorbeeld uitgelokt worden door inspanning, weersomstandigheden, verkoudheid, emoties en blootstelling aan pollen of huisdieren. Hoofdstuk 8 beschrijft dat twee derde van de kinderen met astma één of meerdere uitlokkende factoren van astma aangeeft. De meest voorkomende uitlokkende factoren waren sporten, weersomstandigheden en een verkoudheid of griep. Kinderen met meer uitlokkende factoren ervaarden ook meer beperkingen in de kwaliteit van leven, zowel in de kwaliteit van leven specifiek voor astma als in de algemene kwaliteit van leven. De uitlokkende factoren sporten, weersomstandigheden en emoties hingen samen met een lagere astma-specifieke kwaliteit van leven score. De uitlokkende factoren emoties en eten of drinken hingen samen met een lagere algemene kwaliteit van leven score. Het is aan te 
bevelen om bij kinderen met astma de aanwezigheid van uitlokkende factoren van astmaklachten uit te vragen om vervolgens goede voorlichting te kunnen geven over de vermijding en/of behandeling van (eventuele) uitlokkende factoren.

\section{Aanbevelingen}

Tot slot worden in hoofdstuk 9 de belangrijkste resultaten van dit proefschrift in perspectief geplaatst en worden aanbevelingen gedaan voor toekomstig onderzoek.

Er wordt geconcludeerd dat een beslisregel op basis van sIgE tegen Ara $\mathrm{h} 2$ betrouwbaar en veilig is bij kinderen in Nederland met een verdenking op een pinda allergie. De invoering van de beslisregel in de nationale richtlijn wordt aangeraden, zowel voor kinderen als volwassenen met een verdenking op een pinda allergie. Internationale studies zijn nodig om de beslisregel wereldwijd te onderzoeken en vervolgens wereldwijd in te voeren. Andere vragen die nog beantwoord moeten worden gaan in het bijzonder over het verbeteren van het voorspellen van pinda allergie bij patiënten met een twijfelachtige Ara h 2 uitslag, het voorspellen van de ernst van voedselallergie en de betrouwbaarheid van de beslisregel bij jonge kinderen $(<3.5$ jaar). De laatstgenoemde vraag is toenemend relevant nu de ontwikkelingen op het gebied van het voorkomen van voedselallergie (vroege introductie) en het behandelen van voedselallergie (immunotherapie) zich richten op deze jonge leeftijdscategorie.

Daarnaast wordt geconcludeerd dat het stellen van een duidelijke diagnose van pinda allergie bijdraagt aan het verminderen van de angst en het verbeteren van de kwaliteit van leven van ouders. Aangezien de angst van kinderen en/of ouders de belangrijkste reden was om af te zien van het eten van pinda thuis of van een voedselprovocatietest, dient vervolgonderzoek na te gaan of deze angst verminderd kan worden door bijvoorbeeld psychologische begeleiding.

Evenzeer zou meer inzicht over de kwaliteit van leven bij kinderen met atopische ziekten verkregen kunnen worden door het meten van andere aspecten, zoals weerbaarheid, hoe patiënten omgaan met hun ziekte en door de ontwikkeling van meetinstrumenten bij kinderen onder de leeftijd van 8 jaar. 


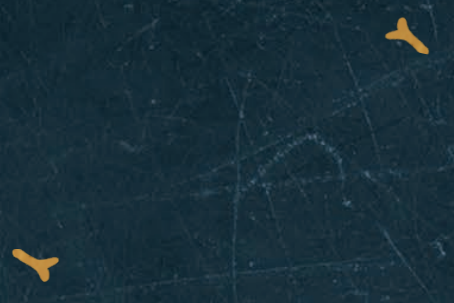

1

1

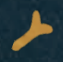

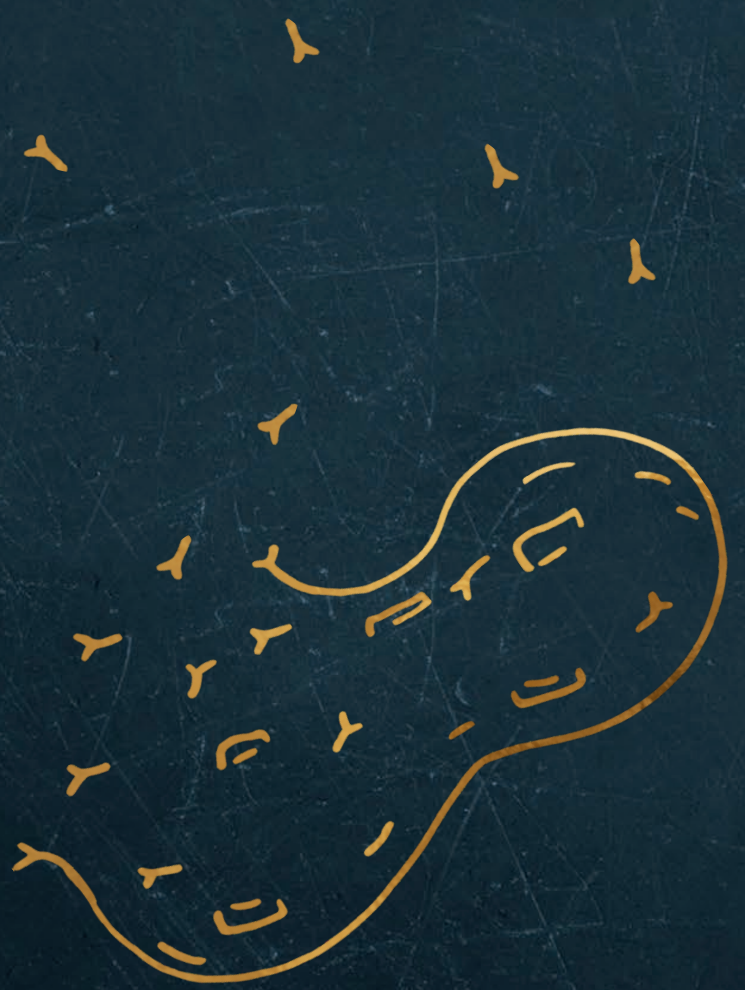




\section{Chapter 11}

List of abbreviations

Contributing authors

Dankwoord

List of publications

Curriculum vitae 


\section{List of abbreviations}

$\begin{array}{ll}\text { ACT } & \text { Asthma control test } \\ \text { ANOVA } & \text { Analysis of variance } \\ \text { AUC } & \text { Area under the curve } \\ \text { BAT } & \text { Basophil activation test } \\ \text { C-ACT } & \text { Child asthma control test } \\ \text { CI } & \text { Confidence interval } \\ \text { CRD } & \text { Component-resolved diagnostics } \\ \text { DBPCFC } & \text { Double-blind placebo-controlled food challenge } \\ \text { ED } & \text { Eliciting dose } \\ \text { EP } & \text { Electronic portal } \\ \text { FAQL-PB } & \text { Food allergy quality of life - parental burden } \\ \text { FAQLQ-AF } & \text { Food allergy quality of life questionnaire - adult form } \\ \text { FAQLQ-CF } & \text { Food allergy quality of life questionnaire - child form } \\ \text { FAQLQ-TF } & \text { Food allergy quality of life questionnaire - teenager form } \\ \text { FAQLQ-PF } & \text { Food allergy quality of life questionnaire - parent form } \\ \text { FC } & \text { Food challenge } \\ \text { HRQL } & \text { Health-related quality of life } \\ \text { InC } & \text { Inconclusive } \\ \text { ING } & \text { Ingredient } \\ \text { ISAAC } & \text { International study of asthma and allergies in childhood } \\ \text { IQR } & \text { Interquartile range } \\ \text { LOAEL } & \text { Lowest-observed-adverse-effect level } \\ \text { MARS } & \text { Medication adherence rating scale } \\ \text { MCID } & \text { Minimal clinically important difference } \\ \text { MD } & \text { Mean difference } \\ \text { N } & \text { Number } \\ \text { NA } & \text { Not applicable } \\ \text { NPV } & \text { Negative predictive value } \\ \text { OFC } & \text { Oral food challenge } \\ \text { OR } & \text { Odds ratio } \\ \text { PAL } & \text { Precautionary allergen labelling } \\ \text { PAQLQ } & \text { Pediatric asthma quality of life questionnaire } \\ \text { PedsQL } & \text { Paediatric quality of life inventory 4.0 } \\ \text { PPV } & \text { Positive predictive value } \\ \text { RAND } & \text { RAND (no abbreviation) general health-rating index } \\ \text { ROC } & \text { Receiver operating characteristic } \\ \text { RRTIs } & \text { Standard devirationy tract infections } \\ \text { SD } & \\ \text { SE } & \text { Stand } \\ & \end{array}$


SIgE

SMD

SPT

STAI

STAIC

UNK

UNP

WHISTLER

WHOQOL-BREF
Specific immunoglobulin E

Standardized mean difference

Skin prick testing

State-trait anxiety inventory

State-trait anxiety inventory for children

Unknown

Unpacked foods

Wheezing illnesses study Leidsche Rijn

World Health Organization generic quality of life scale 


\section{Contributing authors}

\section{Walter A.F. Balemans}

Department of Pediatrics, St. Antonius Hospital, Nieuwegein, The Netherlands

\section{Anna M. Ehlers}

Department of Dermatology/Allergology, University Medical Center Utrecht, Utrecht, The Netherlands \& Center of Translational Immunology, University Medical Center Utrecht, Utrecht, the Netherlands.

\section{Martine van Engelen}

Princess Máxima Centre for Paediatric Oncology, Utrecht, The Netherlands

\section{Cornelis K. van der Ent}

Department of Pediatric Pulmonology and Allergology, Wilhelmina Children's Hospital, University Medical Center Utrecht, Utrecht, The Netherlands

\section{Francine C. van Erp}

Department of Dermatology/Allergology, University Medical Center Utrecht, Utrecht, The Netherlands

\section{Bart E. van Ewijk}

Department of Pediatrics, Tergooi Hospital, Blaricum/Hilversum, The Netherlands

\section{Geert W.J. Frederix}

Julius Center for Health Sciences and Primary Care, University Medical Center Utrecht, Utrecht, The Netherlands

\section{Diana M.W. Gorissen}

Department of Pediatrics, Deventer Hospital, Deventer, The Netherlands

\section{Bertine M.J. Flokstra-de Blok}

General Practitioners Research Institute (GPRI), Groningen, The Netherlands \& University of Groningen, University Medical Center Groningen, GRIAC Research Institute, Groningen, The Netherlands \& Department of Pediatric Pulmonology and Pediatric Allergology, University of Groningen, University Medical Center Groningen, Beatrix Children's Hospital, Groningen, The Netherlands

\section{Willem R. Keusters}

Julius Center for Health Sciences and Primary Care, University Medical Center Utrecht, Utrecht, The Netherlands 


\section{Edward F. Knol}

Department of Dermatology/Allergology, University Medical Center Utrecht, Utrecht, The Netherlands \& Center of Translational Immunology, University Medical Center Utrecht, Utrecht, the Netherlands.

\section{André C. Knulst}

Department of Dermatology/Allergology, University Medical Center Utrecht, Utrecht, The Netherlands \& Center of Translational Immunology, University Medical Center Utrecht, Utrecht, the Netherlands.

\section{Thuy My Le}

Department of Dermatology/Allergology, University Medical Center Utrecht, Utrecht, The Netherlands

\section{Melanie A. Lebbink}

Department of Pediatric Immunology and Infectious Diseases, Wilhelmina Children's Hospital, University Medical Centre Utrecht, Utrecht, The Netherlands

\section{Sarah A. Lyons}

Department of Dermatology/Allergology, University Medical Center Utrecht, Utrecht, The Netherlands

\section{Yolanda Meijer}

Department of Pediatric Allergology, Amsterdam University Medical Center, Amsterdam, The Netherlands

\section{Joeri Mul}

Princess Máxima Centre for Paediatric Oncology, Utrecht, The Netherlands

\section{Henny G. Otten}

Center of Translational Immunology, University Medical Center Utrecht, Utrecht, the Netherlands.

\section{Gordon H.P.R. Slabbers}

Department of Pediatrics, Bernhoven Hospital, Uden, The Netherlands

\section{Marike Stadermann}

Department of Allergology, Diakonessenhuis Utrecht, Utrecht, The Netherlands

\section{Sabine M.P.J. Prevaes}

Department of Pediatric Pulmonology and Allergology, Wilhelmina Children's Hospital, University Medical Center Utrecht, Utrecht, The Netherlands 


\section{Cuno S.P.M. Uiterwaal}

Julius Center for Health Sciences and Primary Care, University Medical Center Utrecht, Utrecht, The Netherlands

\section{Lilly M. Verhagen}

Department of Pediatric Immunology and Infectious Diseases, Wilhelmina Children's Hospital, University Medical Centre Utrecht, Utrecht, The Netherlands

\section{Maartje F. van Velzen}

Department of Pediatrics, Meander Medical Center, Amersfoort, The Netherlands

\section{Esther de Vries}

Department of Tranzo, Tilburg School of Social and Behavioral Sciences, Tilburg University, Tilburg, The Netherlands \& Jeroen Bosch Academy Research, Jeroen Bosch Hospital, 's Hertogenbosch, The Netherlands

\section{Paco M.J. Welsing}

Department of Dermatology/Allergology, University Medical Center Utrecht, Utrecht, The Netherlands 
Contributing authors 


\section{Dankwoord}

De finish van mijn promotie marathon is in zicht! Ik wil op deze plek iedereen bedanken die de afgelopen jaren tijdens de pieken en de dalen heeft meegevierd, meegeleefd of meegeholpen. Een aantal mensen wil ik in het bijzonder bedanken.

Allereerst alle kinderen, ouders en volwassen die hebben meegedaan aan het onderzoek. Bewonderenswaardig en onmisbaar!

Kors en André, bedankt voor het vertrouwen en de mogelijkheden die jullie me de afgelopen jaren hebben geboden.

Kors, ik waardeer je advies, humor en de vrijheid die je me hebt gegeven om zelf aan het roer te staan.

André, bedankt voor je betrokkenheid bij mijn promotietraject en kritische blik. Je enthousiasme voor de voedselallergie werkt(e) aanstekelijk.

Thuy My en Francine, één ding weet ik zeker: zonder jullie steun was dit boekje er niet geweest.

Thuy My, jouw kennis van zaken, prettige manier van samenwerken en Sinterklaasgedichten maken je een steengoede copromotor.

Francine, in jouw voetsporen ben ik getreden. Ik had je voorbeeld, kennis, creativiteit en nauwe betrokkenheid voor geen goud willen missen.

Yolanda, of mijn 'allergie-moeder'. Jouw hart voor de kinderallergologie en voor de patiënten zijn ongeëvenaard. Ik waardeer je aandacht en interesse voor mijn promotie, maar vooral ook voor alles wat daarbuiten speelde.

Leden van de leescommissie, Prof. dr. M.H.M. Wauben, Prof. dr. A.E.J. Dubois, Prof. dr. E.M. van de Putte, Prof. dr. A.B.J. Prakken, Prof. dr. R. van Ree, hartelijk dank voor de tijd die jullie genomen hebben om dit proefschrift te beoordelen.

Alle coauteurs wil ik bedanken voor hun waardevolle bijdragen aan dit proefschrift.

Maartje, Marike en Monique, zonder jullie enthousiasme en klinische kennis was ik niet ver gekomen. Ik kijk met plezier terug op alle panels, waar ook Yolanda aan deelnam ('Is het nou een loopneus of een verstopte neus?'). Ontzettend bedankt. Ook ieder ander die heeft meegewerkt aan het APPEAL onderzoek, met name Joyce en Linda: bedankt!

Afdeling Kinderlongziekten- en allergologie: wat een fijne plek om te werken. Ik zal de verjaardagstaarten, kerstontbijtjes en dagelijkse lunches missen. Bedankt voor 
jullie gezelligheid, hulp en interesse. Ook de afdeling Kameleon en de afdeling Dermatologie/Allergologie wil ik bedanken voor de fijne samenwerking. Myriam, Wilma, Sylvia, Miranda en Jantine jullie deur stond altijd open. Bedankt voor alles!

De krenten in de PhD-pap waren de congressen. Bedankt Yolanda, Eva, Ted, Maaike, Sabine P, Annemieke, Maartje, Jurgen, Frank, Femke, Kim, Olivia, Marike, Dominique, Ralph en de dermatologie-groep. Daphne en Sarah, Lissabon was onvergetelijk.

Lieve mede-promovendi, Peter, Gitte, Maaike, Nienke, Danya, Sabine vd L, Marlou, Maartje, bedankt voor de gezelligheid en vele koffie-, spar-, en klaagmomenten. G02-kamergenoten, Sarah, Mark S, Mark B, Anna, Mehran, Daphne, Lotte, Fleur, Roselie, Lieneke, Judith, Mignon, Henrike, Celeste, bedankt dat ik op jullie kamer toch (soms) een plekje kon krijgen en kon aanhaken bij vele borrels. Edward, Geert, Henny, Stans, Kitty, Heike en Astrid: dank voor jullie 'acute onstekings' inzichten.

Victorien en Roderick, als groentje hebben jullie mij kennis laten maken met onderzoek doen. Ik denk met veel plezier terug aan deze periode die het beginpunt vormde van mijn tijd als arts-onderzoeker.

Iedereen die in de loop der jaren zijn of haar steentje heeft bijgedragen aan het Luchtwegportaal: bedankt! Hannah en Emma, bij jullie kon ik altijd aankloppen voor hulp bij het versturen van Donald Duckjes of andere Luchtwegportaal-zaken. Lilly, onze samenwerking waardeer ik erg.

Studenten, Coco, Lisanne, Francina, Joeri, Melanie, van tientallen patiënten bellen tot enorme databases tackelen, ontzettend bedankt voor jullie hulp en inzet.

Paranimfen, Maaike en Sarah, ik ben heel blij dat jullie naast me staan, als collega's en vriendinnen.

Lieve vrienden, wat moest ik zonder jullie gezelligheid, humor en relativering als welkome afleiding? Het is fijn om te weten dat jullie er voor me zijn.

Renée, Hans, Daniel en Sara, bedankt voor het warme welkom in jullie familie en het verbreden van mijn kennis over katten, koeten en kometen.

Gerard en Lydia, lieve pap en mam. Een fijner en veiliger thuis kan ik me niet voorstellen. Bedankt voor alles. Maarten, je trots op mij als grote zus is ontzettend lief. Bedankt voor je support.

Lieve Erik, je bent onmisbaar. Bedankt voor het avontuur, je humor, (ver)trouw(en) en liefde. Met jou is alles leuker en ik kijk enorm uit naar wat nog komt! 


\section{List of publications}

\section{This thesis, published}

HM Kansen, TM Le, Y Meijer, BMJ Flokstra-de Blok, PMJ Welsing, CK van der Ent, AC Knulst, FC van Erp. The impact of oral food challenges for food allergy on quality of life: a systematic review. Pediatr Allergy Immunol. 2018; 29(5): 527-537.

HM Kansen, TM Le, Y Meijer, CSPM Uiterwaal, AC Knulst, CK van der Ent, FC van Erp. Perceived triggers of asthma impair quality of life in children with asthma. Clin Exp Allergy. 2019; 49(7): 980-989.

HM Kansen, TM Le, AC Knulst, DMW Gorissen, CK van der Ent, Y Meijer, FC van Erp. Three-year follow-up after peanut food challenges: accidental reactions in allergic children and introduction failure in tolerant children. J Allergy Clin Immunol. 2020; 145(2): 705-707.

HM Kansen, TM Le, CSPM Uiterwaal, BE van Ewijk, WAF Balemans, DMW Gorissen, E de Vries, MF van Velzen, GHPR Slabbers, Y Meijer, AC Knulst, CK van der Ent, FC van Erp. Prevalence and predictors of uncontrolled asthma in children referred for asthma and other atopic diseases. J Asthma Allergy. 2020; 13: 67-75.

\section{This thesis, submitted}

Kansen HM, FC van Erp, Y Meijer, DMW Gorissen, M Stadermann, MF van Velzen, WR Keusters, GWJ Frederix, AC Knulst, CK van der Ent, TM Le. A diagnostic flowchart for pediatric peanut allergy using Ara $\mathrm{h} 2$ is accurate, safe and reduces costs. Submitted.

Kansen HM, FC van Erp, AC Knulst, AM Ehlers, SA Lyons, EF Knol, Y Meijer, HG Otten, CK van der Ent TM Le. Accurate prediction of peanut allergy in one third of adults using a validated Ara h 2 cutoff. Submitted.

Kansen HM, MA Lebbink, J Mul, FC van Erp, M van Engelen, E de Vries, SMPJ Prevaes, TM Le, CK van der Ent, LM Verhagen. Risk factors for atopic diseases and recurrent respiratory tract infections in children. Submitted. 


\section{Other publications}

HM Kansen, PF van Rheenen, RHJ Houwen, W Tjon a Ten, GM Damen,

A Kindermann, JC Escher, VM Wolters. Less anti-infliximab antibody formation in Ppediatric Crohn patients on concomitant immunomodulators. $J$ Pediatr Gastroeneterol Nutr. 2017; 65(4): 425-429.

HM Kansen, FC van Erp, EF Knol, B Pontoppidan, Y Meijer, CK van der Ent, AC Knulst. Reply. J Allergy Clin Immunol. 2018; 141(1): 458.

KCJM Kraal*, I Timmerman*, HM Kansen, $C$ van den Bos, J Zsiros, $H$ van den Berg, S Somers, E Braakman, AML Peek, MM van Noesel, CE van der Schoot, M Fiocco, HN Caron, C Voermans, GAM Tytgat. Peripheral stem cell apheresis is feasible post ${ }^{131}$ Iodine-metaiodobenzylguanidine- therapy in high-risk neuroblastoma, but results in delayed platelet reconstitution. Clin Cancer Res. 2019; 25(3): 1012-1021.

* Contributed equally 


\section{Curriculum vitae}

Hannah Kansen was born on the $20^{\text {th }}$ of May 1991 in Hilversum, the Netherlands. She graduated cum laude from secondary school in 2009 (de Passie, Utrecht). In the same year she started her medical training at the University of Utrecht. During medical school, she developed her interest in pediatrics during a clinical internship at the St. Jansdal in Harderwijk and started a research project at the department of Pediatric Gastroenterology of the Wilhelmina Children's Hospital, supervised by Prof. dr. R.H.J. Houwen and dr. V.M. Wolters. In addition, she

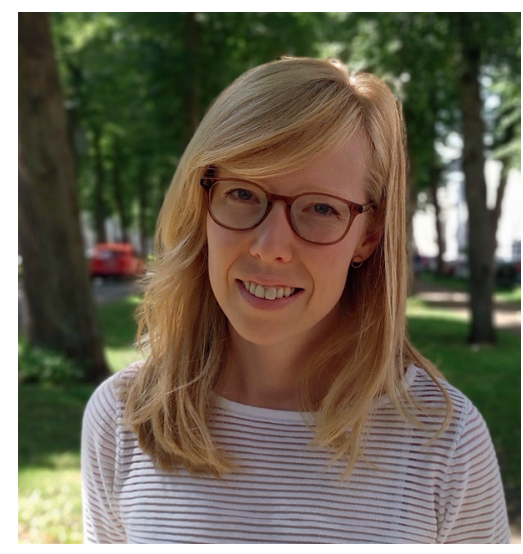
completed a research project at the department of Pediatric Oncology of the Princess Maxima Centre Utrecht, supervised by dr. K.C.J.M. Kraal. Both projects resulted in a publication. She graduated from medical school in 2016 and started her PhD trajectory at the department of Pediatric Pulmonology and Allergology at the Wilhelmina Children's Hospital in Utrecht and the department of Dermatology/Allergology at the University Medical Center in Utrecht, supervised by Prof. dr. C.K van der Ent, Prof. dr. A.C Knulst, dr. T.T.M. Le, dr. F.C. van Erp and drs. Y. Meijer. The results of her PhD research projects have been described in this thesis. During her PhD trajectory, she completed the post-graduate master Epidemiology with specialization in clinical epidemiology at the University of Utrecht. As of July 2020, she works as a resident in pediatrics at the Meander Medical Center Amersfoort. Hannah currently lives in Utrecht with Erik van den Berg. 



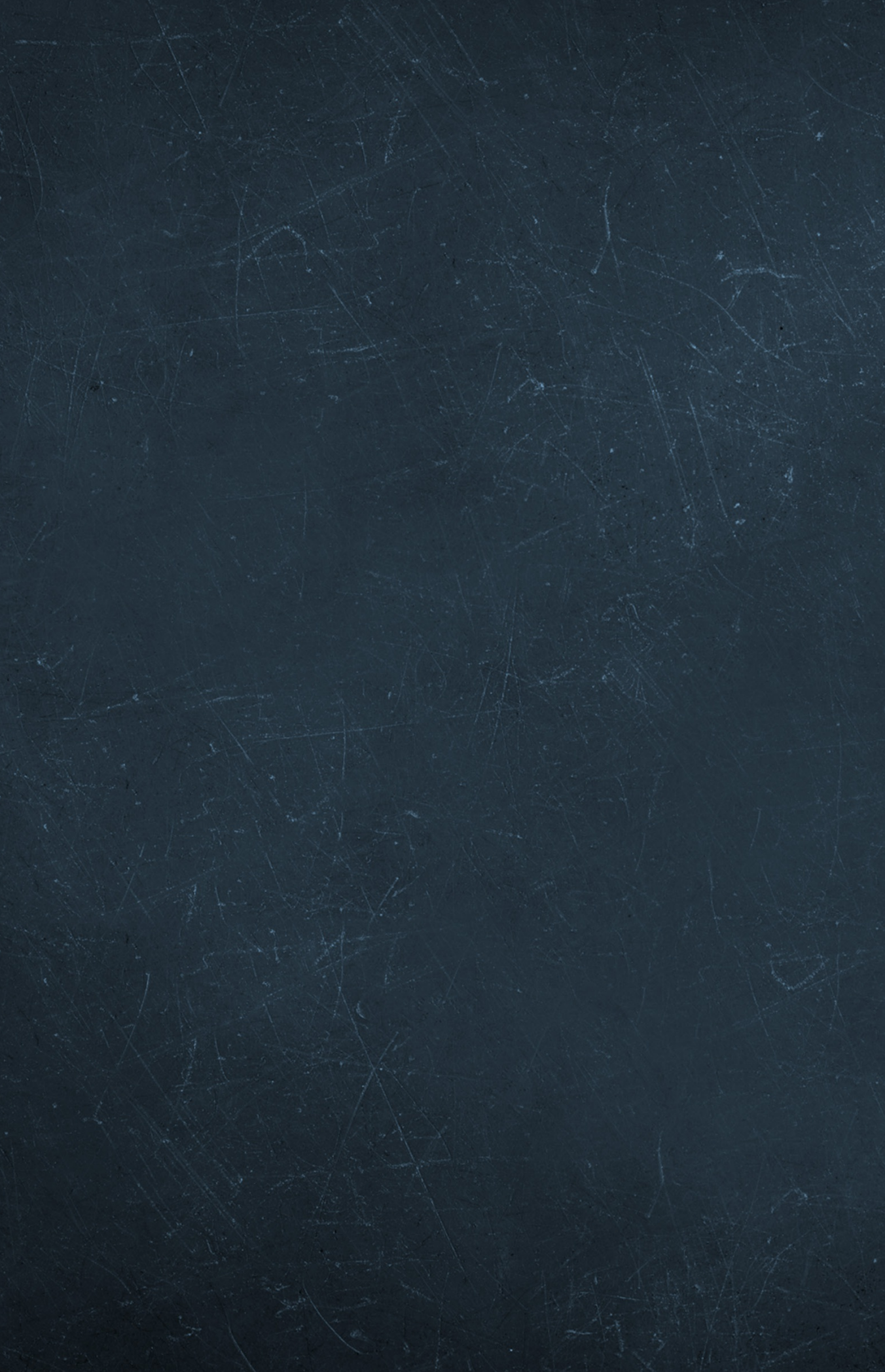

